\title{
Boundary Layer Dynamics and Deep Ocean Mixing in Mid-Atlantic Ridge Canyons
}

\author{
by \\ Rebecca Walsh Dell
}

Submitted in partial fulfillment of the requirements for the degree of

Doctor of Philosophy

at the

MASSACHUSETTS INSTITUTE OF TECHNOLOGY

and the

WOODS HOLE OCEANOGRAPHIC INSTITUTION

February 2013

(c) Rebecca Walsh Dell, MMXIII. All rights reserved.

The author hereby grants to MIT and WHOI permission to reproduce and to distribute publicly paper and electronic copies of this thesis document in whole or in part in any medium now known or hereafter created.

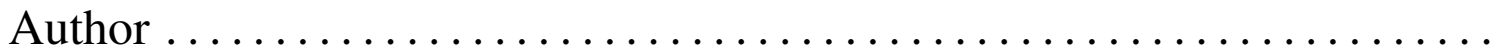

Joint Program in Oceanography - Massachusetts Institute of Technology / Woods Hole Oceanographic Institution

December 12, 2012

Certified by

Lawrence J. Pratt

Senior Scientist

Woods Hole Oceanographic Institution

Thesis Supervisor

Accepted by

Glenn R. Flierl

Professor, Massachusetts Institute of Technology Chair, Joint Committee for Physical Oceanography 


\title{
Boundary Layer Dynamics and Deep Ocean Mixing in
}

\section{Mid-Atlantic Ridge Canyons}

\author{
by \\ Rebecca Walsh Dell
}

Submitted to the Joint Program in Oceanography,

Massachusetts Institute of Technology and the Woods Hole Oceanographic Institution

on December 12, 2012, in partial fulfillment of the

requirements for the degree of

Abstract Doctor of Philosophy

Physical oceanographers have known for several decades the total amount of abyssal mixing and upwelling required to balance the deep-water formation, but are still working to understand the mechanisms and locations-how and where it happens. From observational studies, we know that areas of rough topography are important and the hundreds of Grand-Canyon sized canyons that line mid-ocean ridges have particularly energetic mixing. To better understand the mechanisms by which rough topography translates into energetic currents and mixing, I studied diffusive boundary layers over varying topography using theoretical approaches and idealized numerical simulations using the ROMS model. In this dissertation, I show a variety of previously unidentified characteristics of diffusive boundary layers that are likely relevant for understanding the circulation of the abyssal ocean.

These boundary layers share many important properties with observed flows in abyssal canyons, like increased kinetic energy near topographic sills and strong currents running from the abyssal plains up the slopes of the mid-ocean ridges toward their crests. They also have a previously unknown capacity to accelerate into overflows for a variety of oceanographically relevant shapes and sizes of topography. This acceleration happens without external forcing, meaning such overflows may be ubiquitous in the deep ocean. These boundary layers also can force exchange of large volumes of fluid between the relatively unstratified boundary layer and the stratified far-field fluid, altering the stratification far from the boundary. We see these effects in boundary layers in two- and three-dimensions, with and without rotation.

In conclusion, these boundary layer processes, though previously neglected, may be a source of a dynamically important amount of abyssal upwelling, profoundly affecting predictions of the basin-scale circulation. This type of mechanism cannot be captured by the kind of mixing parameterizations used in current global climate models, based on a bottom roughness. Therefore, there is much work still to do to better understand how these boundary layers behave in more realistic contexts and how we might incorporate that understanding into climate models.

Thesis Supervisor: Lawrence J. Pratt

Title: Senior Scientist

Woods Hole Oceanographic Institution 


\section{Acknowledgments}

First, I thank my advisor, Larry Pratt. He has pushed me while supporting me, and made me a much better scientist. It has also been a delight to share some of his wide-ranging interests in art and performance, and its relationship to science.

My thesis committee has also been extremely helpful throughout this process, challenging my results and enlivening my research. They never let me stay in a rut. My thanks to Tom Peacock, John Toole, Andreas Thurnherr, and Raffaele Ferrari.

I would also like to thank the following people for their invaluable technical assistance throughout the thesis: Julian Schanze, Jinbo Wang, Neil Ganju, Bror Jonsson, Gordon Zhang.

I gratefully acknowledge the financial support of the NSF Graduate Research Fellowship Program and WHOI Academic Programs.

The Academic Programs Office at WHOI and the administrative staff at MIT has made sure that all the students have what we need to thrive. Many thanks.

I have had many wonderful friends and classmates in my time in the Joint Program. My thanks to all of you.

Finally, everything I do in life I owe to my family. They deserve all the credit, I will take all the blame. 


\section{Contents}

$\begin{array}{lr}\text { List of Figures } & 8\end{array}$

1 Introduction $\quad 11$

1.1 Abyssal Canyon Observations _. . . . . . . . . . . . . . 15

1.2 Previous Studies of Boundary Layer Mixing . . . . . . . . . . . . . 21

1.2 .1 Derivation of Simplest Diffusive Boundary Layer . . . . . . . . . 22

1.2 .2 Subsequent Studies of Diffusive Boundary Layers . . . . . . . . 27

1.3 Structure of This Work . . . . . . . . . . . . . . . . . . 29

2 Diffusion-Driven Boundary Layers over Varying Topography, without Rotation 33

2.1 Introduction . . . . . . . . . . . . . . . 33

2.2 Boundary Layers over Broken Slopes _ . . . . . . . . . . . . . . . 36

2.2 .1 Numerical Results . . . . . . . . . . . . . . 36

2.2 .2 Numerical Methods . . . . . . . . . . . . . . . . . . . 37

2.3 Corrugated Slopes . . . . . . . . . . . . . . . . . . . . 40

2.3.1 Description of Numerical Results . . . . . . . . . . . . . 40

2.3 .2 Dependence of Flow Geometry on Topography . . . . . . . . . . . 49

2.4 Predicting Bottom Boundary Layer Geometry . . . . . . . . . . . . . . . . 52

2.5 Conclusions . . . . . . . . . . . . . . . . . . . 59

2.A Appendix - Effects of $\kappa, \nu$ variation on a Constant Slope Boundary Layer . 60 
2.B Appendix - Jet model . . . . . . . . . . . . . . . . . . . 66

2.C Appendix - Studying the Effects of Topography in the Laboratory . . . . 68

3 Diffusion-Driven Boundary Layers over Two-Dimensional Topography, $\begin{array}{ll}\text { with Rotation } & 75\end{array}$

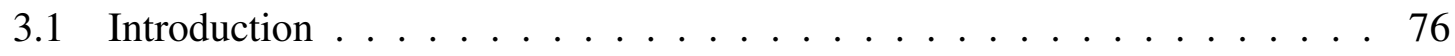

3.2 Numerical Methods . . . . . . . . . . . . . . . . . . . . . . . . 83

3.3 Numerical Results and Interpretation . . . . . . . . . . . . . . 87

3.4 Predicting Flow Geometry with Bottom Stress . . . . . . . . . . . . . 99

3.5 Conclusions . . . . . . . . . . . . . . . . . . . . . 104

4 Diffusion-Driven Boundary Layers in Canyons 107

4.1 Introduction . . . . . . . . . . . . . . . . . . 108

4.2 Numerical Methods . . . . . . . . . . . . . . . . . . . . . 110

4.3 Volume Flux Through the Canyon . . . . . . . . . . . . . 117

4.4 Circulation in a Canyon with a Constant Bottom Slope . . . . . . . . 119

4.4 .1 Dynamics of Sidewall Jets . . . . . . . . . . . . . . . . . . . 129

4.4 .2 Along-Canyon Motion in Time . . . . . . . . . . . . 132

4.5 Circulation in a Canyon with Sills . . . . . . . . . . . . . 137

4.6 Conclusions . . . . . . . . . . . . . . . . . . 145

4.A Appendix - Studying the Canyon Circulation in a Laboratory . . . . . . 147

5 Consequences of Diffusion-Driven Boundary Layers 151

5.1 Novel Contributions of the Thesis . . . . . . . . . . . . . . 151

5.2 Limitations and Opportunities for Future Progress . . . . . . . . . 156

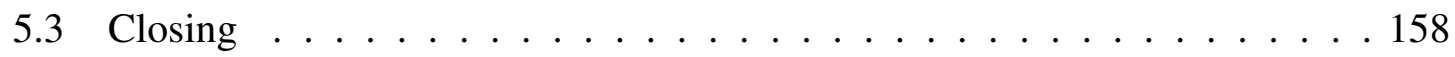

$\begin{array}{ll}\text { Bibliography } & 160\end{array}$ 


\section{List of Figures}

1-1 Effect of spatially varying $\kappa$, from Huang and Jin [2002] . . . . . . . . 12

1-2 $\kappa$ increased over rough topography, from Polzin et al. [1997] . . . . . . . . 14

1-3 Map of canyon locations in the South Atlantic, from A. Thurnherr . . . . 15

1-4 High resolution bathymetry from BBTRE, from Nikurashin and Legg [2011] 16

1-5 Hydrography, currents, and mixing in the $22^{\circ} \mathrm{S}$ canyon, from A. Thurnherr . 18

1-6 Schematic of diffusive boundary layer . . . . . . . . . . . . . 23

1-7 Upslope velocity in a diffusive boundary layer . . . . . . . . . . . . 26

2-1 Velocity field of boundary layer with single change in bottom slope . . . . . 35

2-2 Mass flux into far field as a function of two slopes . . . . . . . . . . . 37

2-3 Velocity field showing exchange over small corrugations on slope . . . . 41

2-4 Density hovmöller over corrugated slope . . . . . . . . . . . . . . . 42

2-5 Momentum balance for small amplitude topography . . . . . . . . . . . . 44

2-6 Momentum balance for large amplitude topography . . . . . . . . . . . . . 44

2-7 Velocity field over corrugations showing breakdown of slowly varying ap-

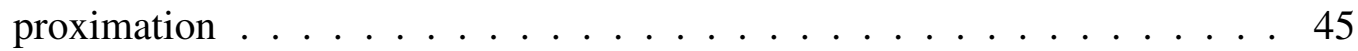

2-8 Overflow generated by boundary layer over corrugations: density and velocity field . . . . . . . . . . . . . . . . . 46

2-9 Streamfunction over large amplitude corrugations . . . . . . . . . . . . 47

2-10 Momentum balance for boundary layer overflow . . . . . . . . . . . . . 48

2-11 Low stratification water in troughs between sills . . . . . . . . . . . 48 
2-12 Three distinct boundary layer geometries over a corrugated sloping bottom . 50

2-13 Corrugated slope boundary layer geometry mapped in non-dimensional parameter space .......................... 51

2-14 Three overflow behaviors over an asymmetrical bump . . . . . . . . . 52

2-15 Along-slope adjustment of diffusive boundary layer . . . . . . . . . . 53

2-16 Schematic of asymmetrical bump calculation . . . . . . . . . . 54

2-17 Predicted boundary layer velocities at peak of asymmetrical bump . . . . 55

2-18 Relationship between estimated bottom stress and the location of the stag-

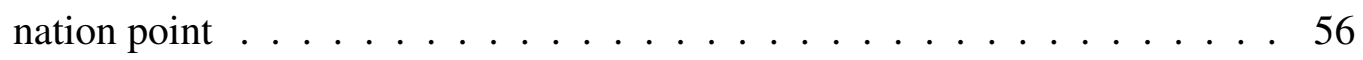

2-19 Bottom shear scaling for multiple bottom slopes . . . . . . . . . . 57

2-20 Schematic for approximating corrugations as asymmetrical bumps . . . . 58

2-21 Comparison between predicted flow geometry and numerical simulation

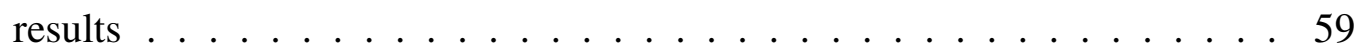

2-22 Numerical and analytical solutions for piecewise constant $\kappa(\eta)$ and $\nu(\eta)$. . 64

2-23 Numerical solutions for smoothly varying $\kappa(\eta)$ and $\nu(\eta) \ldots 65$

2-24 Neutrally-buoyant jet approaches steady state . . . . . . . . . . . 67

2-25 Consistent scaling in the shape of multiple neutrally-buoyant jets . . . . . 68

2-26 Proposed laboratory experiments in nondimensional parameter space . . . . 72

2-27 Overflow boundary layer on high aspect ratio topography . . . . . . . . 73

3-1 Analytical solutions for constant slope diffusive boundary layer with multiple values of $f \ldots \ldots \ldots \ldots$. . . . . . . . . . . . 81

3-2 Schematic of rotating two-dimensional numerical simulation domain . . . 83

3-3 Hovmöller showing two-dimensional rotating simulations at steady state . . 86

3-4 Rotating two-dimensional momentum balance over corrugated slope . . . . 90

3-5 Streamfunctions for corrugated slope with five values of $f / S \ldots \ldots 2$

3-6 Rotating corrugated slope boundary layer flow geometries in non-dimensional parameter space . . . . . . . . . . . . . . . . . . . 94

3-7 Far field stratification modified by rotating corrugated slope boundary layers 95 
3-8 Density field comparison between strong rotation and no rotation . . . . . 97

3-9 Attempted prediction of boundary layer flow geometry from bottom stress at sill . . . . . . . . . . . . . . . . . . . . . . 103

3-10 Size of error from separating buoyant and rotating boundary layers . . . . 105

4-1 Schematic of canyon numerical solution domain . . . . . . . . . . 111

4-2 Velocity profiles from multiple locations in canyon domain . . . . . . . 113

4-3 Effect of parametrized turbulence on trajectories . . . . . . . . . . 116

4-4 Schematic of dynamical regimes in canyon . . . . . . . . . . 120

4-5 Eulerian velocity field and isopycnals in canyon . . . . . . . . . . 122

4-6 Volume fluxes in and above viscous boundary layer in canyon . . . . . 123

4-7 Lagrangian trajectories in canyon with constant bottom slope . . . . . . 124

4-8 Changes in density across a Lagrangian trajectory . . . . . . . . . 126

4-9 Momentum balance for canyon currents . . . . . . . . . . . . 128

4-10 Scaling of sidewall jet width in canyon . . . . . . . . . . . . 132

4-11 Average along-canyon progress of water parcels . . . . . . . . . . 134

4-12 Tracer distribution map from Ledwell et al. [2000] . . . . . . . . . . 136

4-13 Lagrangian trajectories in canyons with sills of varying height $\ldots \ldots 138$

4-14 Velocity fields showing exchange with far field at sill . . . . . . . . 141

4-15 Example of Lagrangian trajectories separating at sill . . . . . . . . . . 143

4-16 Average trajectory separation at sill . . . . . . . . . . . . . . 144

4-17 Schematic of canyon laboratory experiment . . . . . . . . . . . 149

5-1 BBTRE $22^{\circ} \mathrm{S}$ canyon along-axis depth $\ldots \ldots \ldots \ldots \ldots \ldots$ 


\section{Chapter 1}

\section{Introduction}

The job of the physical oceanographer is to give an accounting of how the ocean workshow inputs like heat, kinetic energy, and fresh water move through the system and transform it, what mechanisms are involved, and where those inputs eventually end up. The ultimate goal is a complete, predictive accounting — as a mechanic might give of an engine or a physicist might give of a single hydrogen atom. That goal remains as 'ultimate' as ever, still over the horizon as we puzzle through this system of seemingly infinite complexity. This thesis represents my small contribution to that accounting, offering an insight or two on the role of boundary layers and topography in the circulation and mixing of the abyssal ocean.

Mixing, or the diffusion of mass, is a key component in the ocean's energetics; it is the process by which kinetic energy is transformed into potential energy by bringing denser fluid up from below and combining it with the lighter overlying fluid, raising the center of mass of a stratified water column. It is closely related to the dissipation of kinetic energy, and its irreversible transformation into heat. There are several estimates available of the total diffusion of mass either in the whole ocean [Munk, 1966] or in individual ocean basins [Hogg et al., 1982]. Wunsch and Ferrari [2004] assembled an estimate of the total dissipation of kinetic energy for the whole ocean.

The inputs of kinetic energy are relatively well known: winds and tides. However, 
efforts to describe the distribution and mechanisms of its diffusion and dissipation-by observational, numerical, or theoretical means - have met with considerable difficulty. Direct measurements of diffusion in the open ocean have generally produced diffusivities ten times smaller than the values predicted in the integral budgets: $10^{-5} \mathrm{~m}^{2} \mathrm{~s}^{-1}$ instead of $10^{-4} \mathrm{~m}^{2} \mathrm{~s}^{-1}$ [Ledwell et al., 1993, Gregg, 1987]. This implies that the mixing must be spatially variable. This spatial variability is potentially of primary importance. Huang and Jin [2002] demonstrated using a general circulation model that different distributions of mixing with the same total amount of mixing can produce dramatically different large-scale circulations. An Atlantic basin with enhanced mixing over rough topography and reduced mixing over smooth topography had a strong northward current along the west flank of the Mid-Atlantic Ridge. In the same basin with a constant diffusivity, that current disappeared (Figure 1-1).

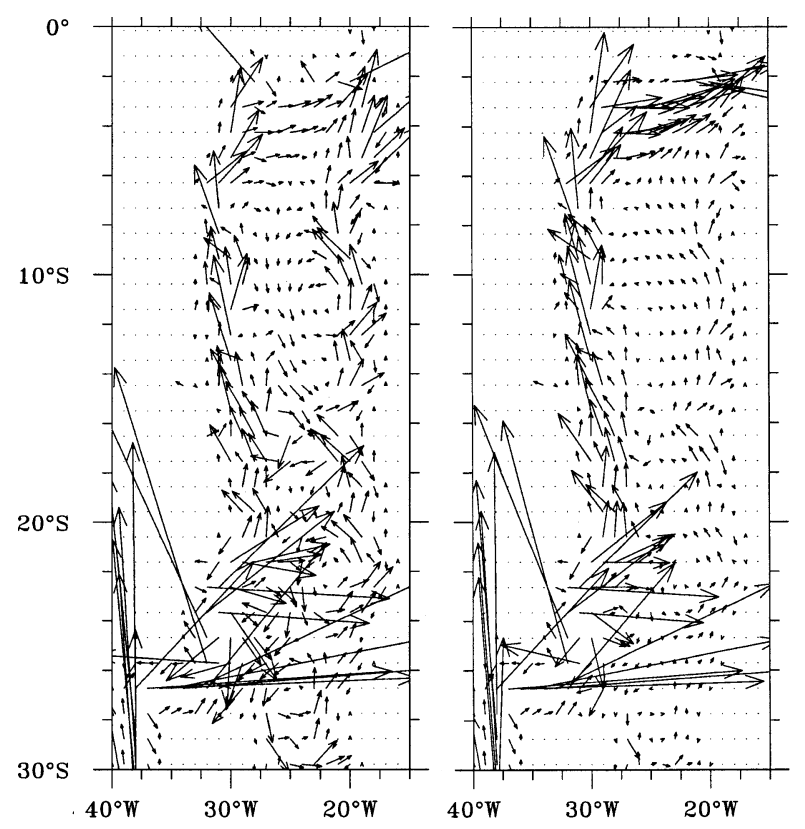

Figure 1-1: Spatially varying diffusivity $\kappa$ produces significantly different abyssal circulation from uniform $\kappa$. In the left panel, we see the circulation in the Brazil Bazin at $4 \mathrm{~km}$ depth in a simulation with higher diffusivity over rough topography, $\kappa=0.1-10 \mathrm{~cm} \mathrm{~s}^{-1}$. On the right is the same field from a simulation with spatially uniform $\kappa=0.85 \mathrm{~cm} \mathrm{~s}^{-1}$. The strong current along the West flank of the Mid-Atlantic Ridge in the left panel disappears on the right. Reproduced from Huang and Jin [2002]. 
This spatial variability of diffusivity is seen directly in observations. Polzin et al. [1997] reported diffusivities increased by one hundred times or more over rough bottom topography compared to the smooth abyssal plain of the South Atlantic (Figure 1-2). Garabato et al. [2004] inferred a similar increase in the Southern Ocean, where the Antarctic Circumpolar current flows through the Drake Passage and over the rough topography of the Scotia Sea. Many observations suggest that the strongest mixing happens around large and complicated topography, especially when there are overflows or flows through topographic constrictions. Polzin et al. [1996] measured dissipation rates above $10^{-6} \mathrm{~W} \mathrm{~m}^{-1}$ immediately downstream of a sill in the Romanche Fracture Zone-10,000 times higher than the typical abyssal background level of $10^{-10} \mathrm{~W} \mathrm{~m}^{-1}$. Overflows have shown such strong mixing that Bryden and Nurser [2003] postulated that all of the mixing in the deep ocean could be accounted for by just a few abyssal straits and overflows.

The canyons of the world's slow-spreading mid-ocean ridges are an intriguing candidate for where abyssal mixing and upwelling may be happening. The sparse observations available in these deep canyons show enhanced turbulent diffusivities and dissipation. They also show significant upslope flows. Together, these canyons may contribute a dynamically important amount of mixing and upwelling in the deep ocean. The mechanism behind this canyon mixing and upwelling is still to be determined.

Inspired by the observations taken in these canyons, this thesis asks some basic questions about the physics of stratified fluids, and applies what we learn about them to better understand the circulation of the deep ocean. I study an idealized model system: a viscous, diffusive bottom boundary layer over varying bottom topography. This system captures several key dynamical features observed in mid-ocean ridge canyons, including isopycnals that bend and intersect the bottom, and bottom-intensified mean flows. At the same time, it is simple enough to be understood much more completely than ocean observations.

In the remainder of this introductory chapter, I will review the observations from abyssal canyons that inspired this work, pointing out some of the most salient features. Then I will review the boundary layer system I study in this thesis, including discussing the extant 


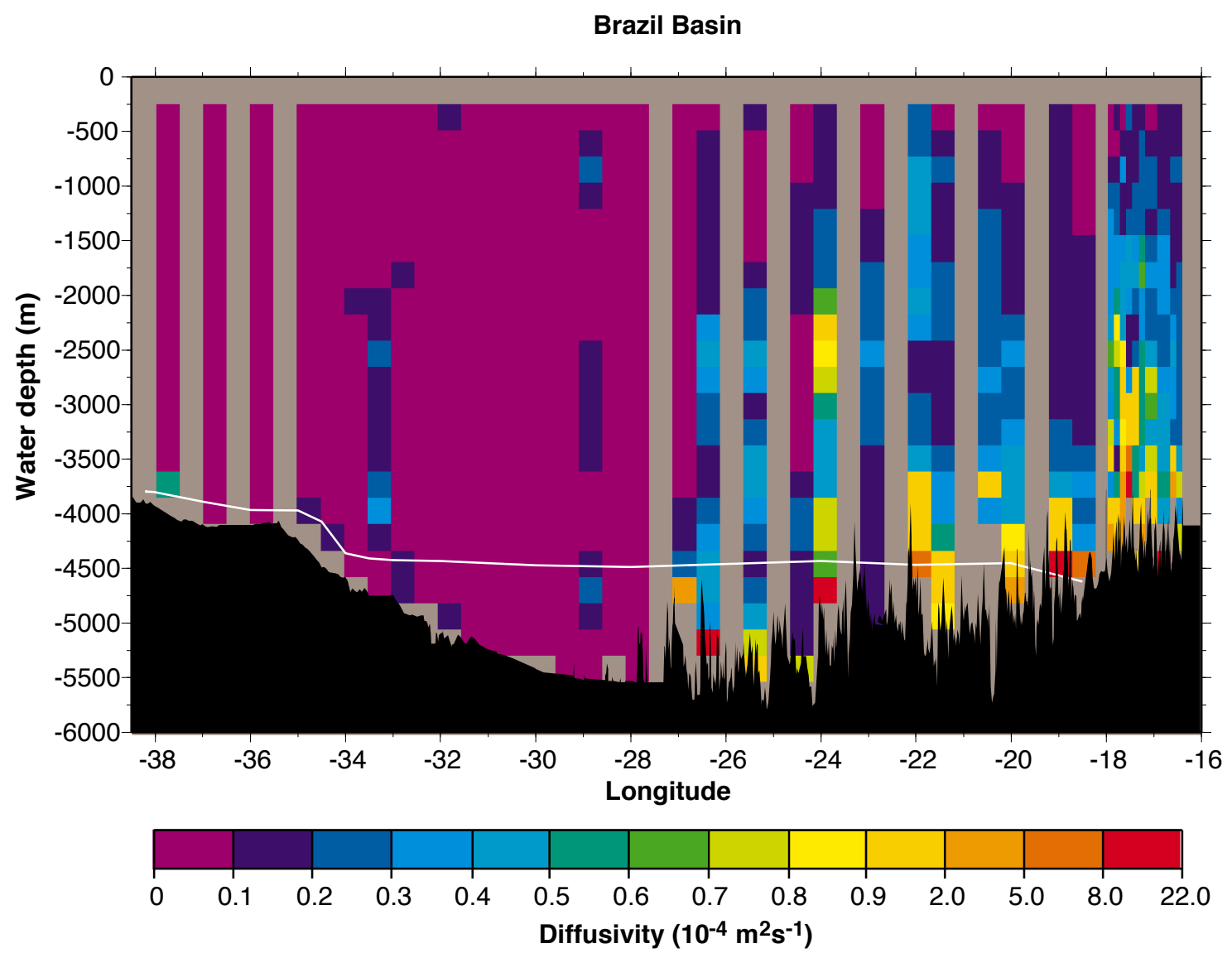

Figure 1-2: Velocity-microstructure-based estimates of turbulent diffusivity show $10-100 \times$ higher mixing over rough topography than over smooth topography. The left (western) portion of the figure is over the smooth abyssal plain of the Brazil Basin, while the right (eastern) portion of the figure is over the much rougher topography of the MidAtlantic Ridge flanks and canyons. Note the non-linear color map. Reproduced from Polzin et al. [1997].

literature and providing a detailed derivation of the simplest boundary layer case. Understanding this derivation will be helpful in understanding the subsequent chapters, so I will be complete. Finally, I will outline the approach taken in the original work in the thesis, and anticipate some of the key results that come out of it. 


\subsection{Abyssal Canyon Observations}

As this work is concerned with processes inside canyons on the ocean bottom, the relevant observations are sparse-suggestive rather than conclusive. That being said, a growing body of observations point to some key features that are present in both ridge-flank canyons and ridge-crest canyons in the Mid-Atlantic Ridge. These canyons share some typical physical features: they tend to be about 20-30 km wide, $1000 \mathrm{~km}$ or more long, and $1 \mathrm{~km}$ deep from the bottom to the top of the canyon walls. The ridge-flank canyons, perpendicular to the ridge crest, are spaced approximately every $100 \mathrm{~km}$ along the length of all slow-spreading ridges (Figure 1-3). This means there are on the order of 1,000 such canyons throughout the world's oceans.

Inside the canyons, there are also consistent features. First, the along-canyon bottom slopes are on the order of $10^{-3}-10^{-2}$. Second, all the canyons are cut by sills or ob-

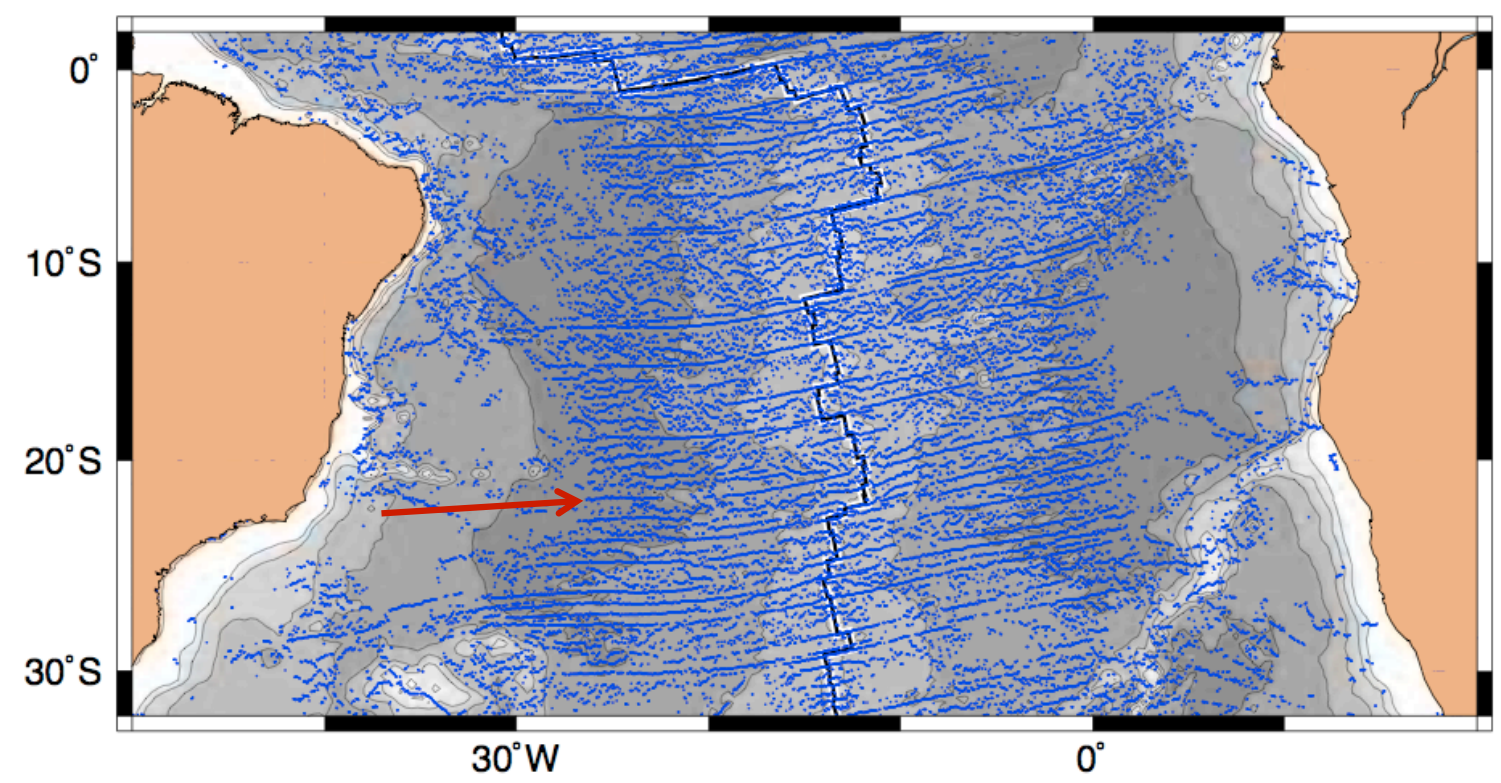

Figure 1-3: Canyons are ubiquitous on the bottom of the Atlantic ocean. In this map, each blue dot represents a local depth maximum. The roughly East-West lines of dots are the ridge flank canyons. The canyon indicated with the red arrow is the $22^{\circ} \mathrm{S}$ canyon, surveyed in the BBTRE. It is very similar to most all of the other Mid-Atlantic Ridge flank canyons. Data from that canyon are shown in several figures throughout the thesis. Courtesy of A. Thurnherr. 
structions every $50 \mathrm{~km}$ or so. These sills stand $200-800 \mathrm{~m}$ tall, and remarkably uniform in the cross-canyon direction, obstructing the full width of the canyon, as shown in high resolution bathymetry measurements taken in the $22^{\circ} \mathrm{S}$ canyon mapped during the Brazil Basin Tracer Release Experiment (BBTRE). (Figure 1-4) The centerline depth of the same canyon is shown in the thick black line of Figure 1-5, to give an idea of the relative height of the sills. Third, most of the canyons, especially ridge-flank canyons and including the $22^{\circ} \mathrm{S}$ canyon, have no outlet: they end in a dead end at the Mid-Atlantic Ridge crest.

At low-and mid-latitudes, the canyons are significantly narrower than both the barotropic and the first baroclinic Rossby radii. The barotropic Rossby radius, $N H / f$, given by an abyssal stratification of $N=3 \times 10^{-3} \mathrm{~s}^{-1}$, a water depth of $H=5 \times 10^{3} \mathrm{~m}$, and a Coriolis

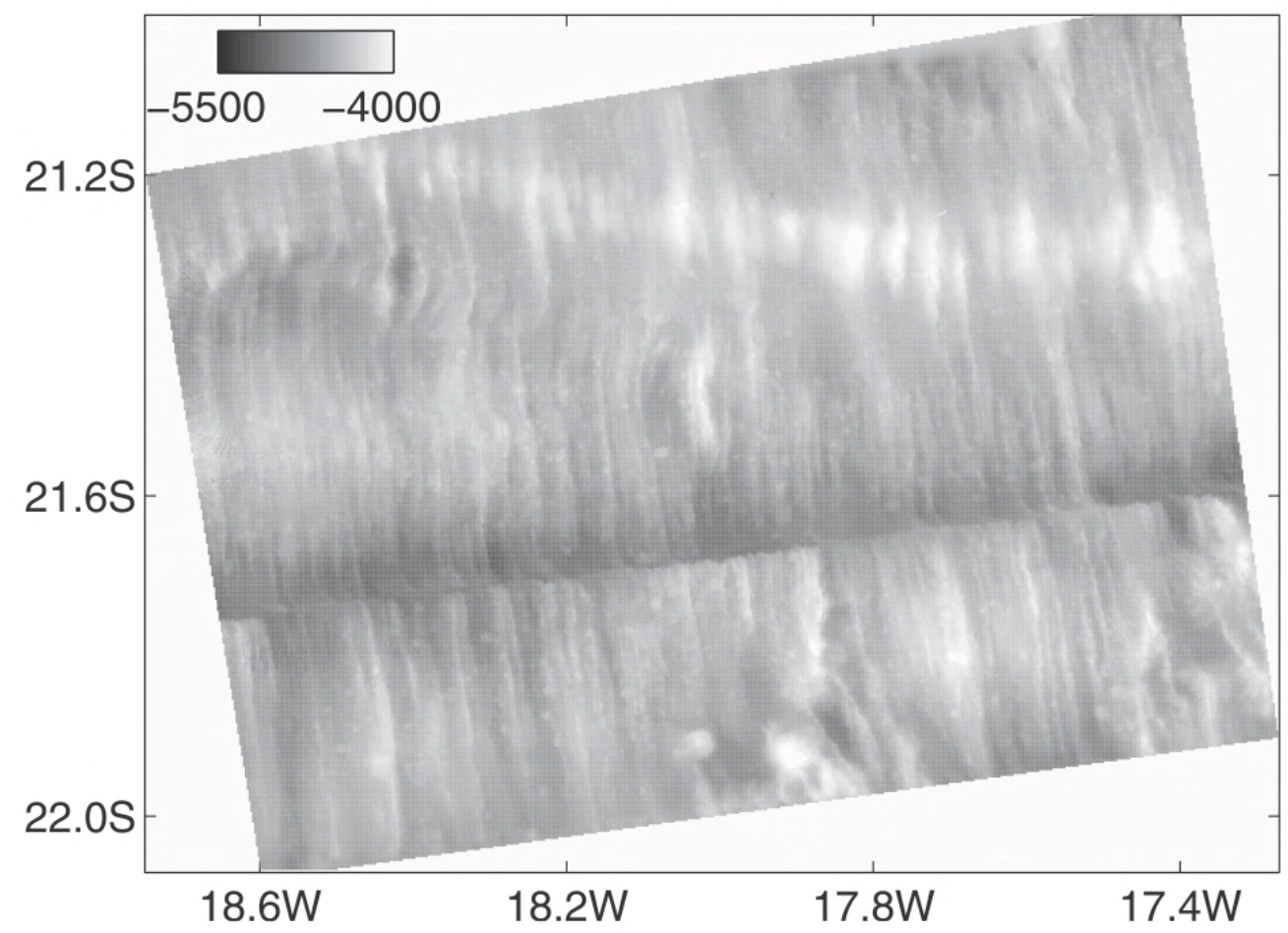

Figure 1-4: High resolution bottom topography from the $22^{\circ} \mathrm{S}$ BBTRE study site. The canyon is approximately East-West and the vertical striations are the sills. The BBTRE current meter was deployed in the canyon at approximately $18 \mathrm{~W}$. Reproduced from Nikurashin and Legg [2011]. 
parameter of $f=10^{-4} \mathrm{~s}^{-1}$ is $L_{R}>100 \mathrm{~km}$. The first baroclinic Rossby radius $R$ is given by Chelton et al. [1998]:

$$
R=\left|\frac{c}{f}\right|
$$

where $c$ is the internal gravity wave speed of the first baroclinic mode and $f$ is the Coriolis parameter. This gives $R$ around $50-60 \mathrm{~km}$ for the $22^{\circ} \mathrm{S}$ canyon studied in the BBTRE. This means that the canyons are narrower than the characteristic geostrophic eddies. However, as we will see in Chapter 4, that does not mean that rotation is unimportant in the dynamics of the canyon.

Within these broadly similar physical settings, the hydrography and currents in deep canyons also exhibit some robust features. In particular, they show along-axis uphill bottom currents, isopycnals that bend down in the along-axis direction and intersect the bottom, strongly increased mixing, and an apparent colocation of strong turbulence with large topographic features. I will discuss these features in turn.

First, all the canyons that have been observed appear to have significant time-average flows along the canyon axis. These mean flows generally contain more energy per unit volume than the tidal frequency bands and often have velocities exceeding the peak tidal velocities. For example, in the ridge-flank canyon at $22^{\circ} \mathrm{S}$ shown in Figure 1-5, a current meter mooring deployed for two years found a mean along-axis current of $1.7 \mathrm{~cm} \mathrm{~s}^{-1}$ going up the slope of the Mid-Atlantic Ridge [Toole, 2007]. This should be compared to a typical peak tidal velocity measure by the same current meter on the order of $2 \mathrm{~cm} \mathrm{~s}^{-1}$. Another canyon in the Lucky Strike segment of the Mid-Atlantic Ridge had a mean alongcanyon velocity of $10 \mathrm{~cm} \mathrm{~s}^{-1}$ over a two-week deployment of an Acoustic Doppler Current Profiler [Thurnherr et al., 2008]. A third canyon near the Azores had a mean velocity around $5 \mathrm{~cm} \mathrm{~s}^{-1}$ with peak tidal velocities of $3 \mathrm{~cm} \mathrm{~s}^{-1}$ [Keller et al., 1975]. These alongaxis flows have been reported in canyons in a variety of orientations and hydrographic settings, suggesting that they arise from processes within the canyon, and not from largerscale pressure gradients or other exogenous factors. Furthermore, the currents measured tend to be strongly bottom-intensified and confined within the canyon, as shown by the red 
arrows in Figure 1-5. In the BBTRE canyon, the largest mean velocities were within $200 \mathrm{~m}$ of the bottom, while above the canyon walls the measured velocities were not significantly different from zero.

Second, the canyons tend to show isopycnals bending downward and intersecting the bottom. In the $22^{\circ} \mathrm{S}$ canyon, the isopycnals (shown in color in the upper panel of Figure 1-5) were flat above the canyon walls (shown as thin white lines in the same figure), but significantly sloped within the canyon. This means that along-canyon currents are also diapycnal currents.

Third, consistent with the water mass transformation implied by the bending isopycnals, ocean bottom canyons all seem to have highly increased levels of mixing. In the canyon studied in the BBTRE, the mean dissipation level based on velocity microstructure was estimated to be $9.3 \times 10^{-10} \mathrm{~W} \mathrm{~kg}^{-1}$ and the typical diffusivity was $4.3 \times 10^{-3} \mathrm{~m}^{2} \mathrm{~s}^{-1}$ [Thurnherr et al., 2005], and the study participants believe that mixing in the canyon may have been systematically undersampled [Ledwell et al., 2000]. These values should be compared to the above cited background dissipation rate in the ocean at mid-depth of $10^{-10} \mathrm{~W} \mathrm{~kg}^{-1}$, supporting a turbulent diffusivity of $10^{-5} \mathrm{~m}^{2} \mathrm{~s}^{-1}$. Both the diffusivity and the dissipation rate are enhanced by an order of magnitude or more in the canyons. Thurnherr et al. [2005] and Morris et al. [2001] both classify the geography of the Brazil Basin and Mid-Atlantic Ridge flank by topographic features: abyssal plain, ridge flank,

Figure 1-5 (facing page): Hydrographic section from the Brazil Basin Tracer Release Experiment, with mean velocities measured by a current meter mooring. These data were taken along the Mid-Atlantic Ridge flank canyon at $22^{\circ} \mathrm{S}$. In the upper panel, rainbow contours show density, the thick black line gives the high resolution multi-beam bathymetry along the canyon axis, and the faint white lines are the tops of the canyon walls. Notice how the lines of constant density curve down and intersect the topography along the ridge slope, implying a west-to-east pressure gradient. The red arrows show the mean velocity as measured by a current meter mooring deployed for approximately two years [Toole, 2007]. In the lower panel, the horizontal blue bars give the turbulent dissipation. The blue numbers are depth-averaged dissipation in units of $10^{-10} \mathrm{~W} \mathrm{~kg}^{-1}$, with the upper numbers covering depths above the canyon walls and the lower numbers covering the depths below. Courtesy of A. Thurnherr. 


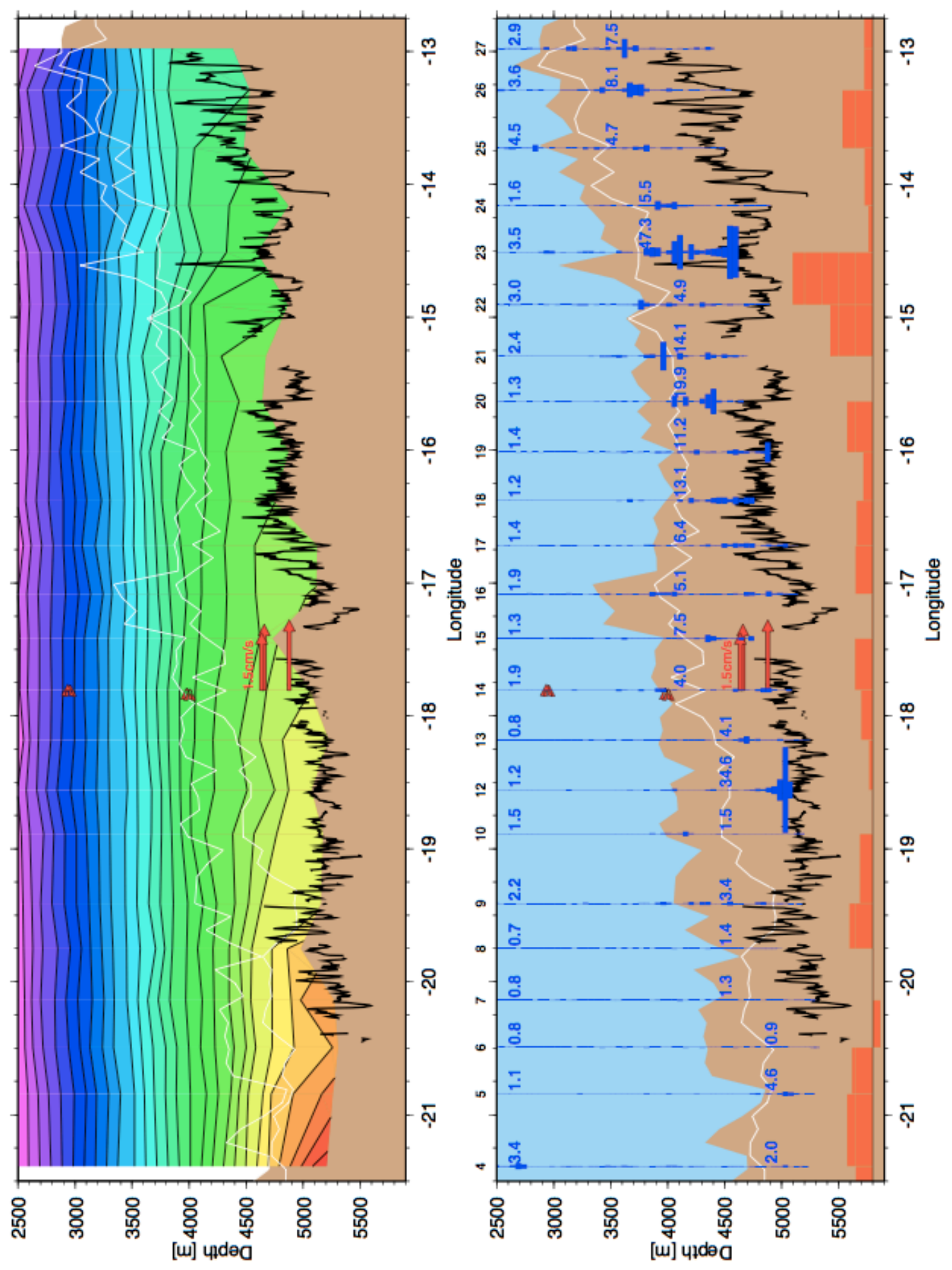


and canyons. Using microstructure data from the BBTRE, they assign typical amounts of dissipation to the different classes. Based on these typical dissipation profiles, Thurnherr et al. find that as much as half of all the turbulent dissipation in the abyssal Atlantic may be happening in canyons; they occupy only $15 \%$ of the area, but have mixing levels enhanced by an order of magnitude above the background (Table 1.1). Morris et al. find that this topographic classification yields results consistent with this and with estimates of mixing based on the heat budgets like those in Hogg et al. [1982].

A fourth notable feature of these canyon flows is the apparent colocation of the highest levels of mixing and the topographic sills that obstruct the along-canyon current. The often sparse data suggests that there may be spilling flows over these sills, and possibly even instances of hydraulic control [Thurnherr et al., 2005, St. Laurent and Thurnherr, 2007]. Thurnherr et al. find that the along-canyon horizontal density gradient is larger near sills, indicating additional mixing. An example of this colocation is the huge increase in dissipation measured just downstream of an $800 \mathrm{~m}$ tall sill at about $14.5 \mathrm{~W}$ in the $22^{\circ} \mathrm{S}$ canyon (Figure 1-5, lower panel). The small blue numbers in the figure give the depth-averaged dissipation below the canyon walls in units of $10^{-10} \mathrm{~W} \mathrm{~kg}^{-1}$.

Observations of mixing in the deep ocean-both those cited above and other studies that did not include canyons-have attracted sustained interest in the physical oceanography community for at least the last twenty years. Currently, the preferred mechanism invoked

\begin{tabular}{lccc} 
Region & Area & Dissipation $\left(\mathrm{W} \mathrm{kg}^{-1}\right)$ & Mixing \\
\hline Abyssal Plain & $40 \%$ & $0.9 \times 10^{-10}$ & $14 \%$ \\
Above MAR flank & $45 \%$ & $1.9 \times 10^{-10}$ & $33 \%$ \\
Inside Canyons & $15 \%$ & $9.3 \times 10^{-10}$ & $53 \%$
\end{tabular}

Table 1.1: Relative contributions to abyssal mixing by various types of topography in the Brazil Basin. For each class of topography, the area covered by that class is given, as is the mean diffusivity observed over that class of topography, and the percent of the total diffusivity in the Brazil Basin that represents. The proportion of mixing in each region shown in the final column is simply the normalized product of the area and the dissipation. Data from Thurnherr et al. [2005], Table 1, based on BBTRE microstructure surveys. These data reflect only mixing below $2000 \mathrm{~m}$ depth. 
to explain the mixing observations is internal waves generated by tides, which propagate away from topography and then steepen and break through nonlinear interactions (for a review, see Garrett and Kunze, 2007). This explanation has also been specifically applied to the BBTRE data by Polzin [2009] and Nikurashin and Legg [2011]. However, this explanation does not account for some important features of the canyon observations. For starters, where does the along-canyon mean flow (found in every canyon where anyone has looked) come from? As it can carry as much or more energy than the tides, how is it affected by topography? And perhaps most curious, how would a homogeneous internal wave field explain why mixing inside the canyon is so much stronger-five times stronger-than above the equally rough flanks of the Mid-Atlantic Ridge?

If the observations described here are typical of abyssal canyons generally, mixing in abyssal canyons could have potentially large implications for the way we understand and model ocean dynamics. Internal waves are undoubtably important, but these observations indicate that the more direct topographic interactions involved in canyon-mixing, like overflows, constrictions, and possibly hydraulic control, may be significant as well. These are very different than the mechanisms, with very different associated mixing distributions. If our understanding of the mechanisms and location of deep ocean mixing changes, our understanding of the ocean's energy budget must change with it.

Now that I have reviewed the observations of abyssal canyons, I will describe the boundary layer ideas that form the foundation of this thesis.

\subsection{Previous Studies of Boundary Layer Mixing}

The general idea of boundary mixing - that the majority of all abyssal mixing might be happening in relatively thin layers near the boundary-is not a new one. Munk [1966] mentioned it in Abyssal Recipes. Perhaps the most scientifically eloquent case for boundary mixing was made by Armi [1978]. He used a combination of observations of layered structures in Brazil Basin hydrography, back-of-the-envelope calculations, and isotope 
observations from fast-decaying elements found on the sea floor to make a compelling case that mixed water from the bottom boundary layer was being exchanged with water over the abyssal plain, and that this exchange could account for the vertical mixing that is traditionally accounted for as a straightforward vertical diffusivity. Even though boundary mixing is not a new idea, as Garrett [2003] says, "There are still many basic questions about the physical processes associated with the influence of all scales of bottom topography."

\section{2 .1 Derivation of Simplest Diffusive Boundary Layer}

One class of boundary mixing problems that is well-studied, and forms the jumping-off point of this thesis, is diffusive boundary layers over constant-slope bottoms. The simplest version of this is the viscous, diffusive boundary layer over an infinite constant slope bottom, first described separately but simultaneously by Phillips [1970] (hereafter referred to as Phillips) and Wunsch [1970]. This case is worth going through in detail, as so much of what follows will back to it. Here, and in the rest of the thesis, I will largely follow the notation from Phillips.

Before deriving the analytical solution, let's consider the physics of a constant stratification fluid over an insulating sloping bottom. If the fluid is temperature stratified, there is a constant downward heat flux due to molecular diffusion throughout the domain. When this heat flux reaches the bottom, it cannot continue through the insulating boundary and therefore it accumulates. The water parcel at the bottom grows warmer, while an adjacent water parcel that is at the same depth but in a deeper part of the domain (so not at the boundary) does not. This sets up a horizontal density gradient, which in turn implies a horizontal pressure gradient. This pressure gradient drives an upslope flow. The heat flux convergence at the boundary is then perfectly compensated by the advection of colder, deeper water along the sloping bottom. Another way to think about the same idea is that the insulating bottom boundary means that isopycnals must intersect the bottom at a right angle-fluxes go down gradient, so in order to have no flux at the bottom, the gradient there must go to zero, as illustrated in Figure 1-6. The natural consequence of these dynamics looks very much like 


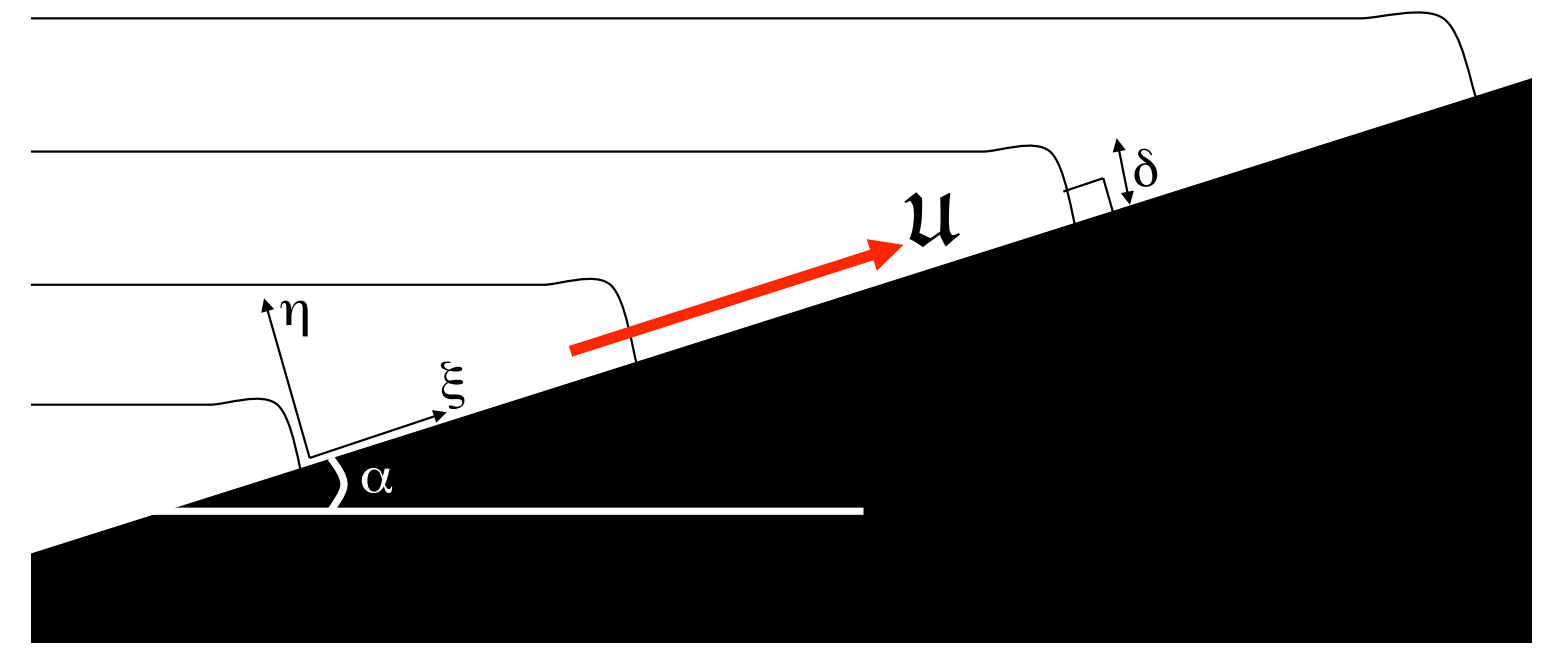

Figure 1-6: Schematic diagram of diffusion-driven flow. The thin black lines are surfaces of constant density, which curve downward over a thickness scale $\delta$ to intersect the slope at a right angle. This produces a left-to-right pressure gradient, driving the up-slope flow, $\mathfrak{u}$, indicated by the red arrow. For constant or slowly varying slopes, it is useful to use slope-normal coordinates $(\xi, \eta)$.

what we saw in the abyssal canyons discussed in the previous section. Here is a mechanism for bottom-intensified mean flows going uphill along a sloping bottom and for isopycnals bending downward to intersect the bottom. What remains to be seen is how such a system would respond to topography; that is the central question of this thesis.

Toward that end, let's make our physical intuition about this system a little more precise. Under the simplifying assumptions of constant bottom slope $\alpha_{0}$, constant background stratification $N$, and infinite extent, the most natural coordinates to use are an along-slope coordinate $\xi$ and a slope-normal coordinate $\eta$, instead of horizontal and vertical coordinates (illustrated in Figure 1-6). Throughout this thesis, I will use $(x, \xi, \eta)$ as the along-isobath, upslope, and slope-normal coordinates, with the associated velocities $(u, v, w)$. On occasion, I will use standard gravitationally-aligned coordinates $(x, y, z)$. The $x$ coordinate is the same in both cases.

In the rotated coordinate system, the problem reduces to a one-dimensional system that varies only in the slope-normal $\eta$ direction. $\frac{\partial}{\partial \xi}=0$ for all boundary layer variables. 
However, the density $\rho$ and pressure $p$ will still contain a term that varies with $\xi$ because the density and pressure vary with the background stratification. Since there is no along-slope variation in the along-slope velocity, the no-normal-flow boundary condition at the bottom boundary implies that the slope-normal velocity $w=0$ throughout the domain. Because of this, and because $u$ is independent of $\xi$, the nonlinear advection terms are precisely zero, so it is not necessary to assume linearity in the constant-slope, constant-stratification case. Finally, we seek a steady-state solution, where $\frac{\partial}{\partial t}=0$, and we assume the mixing coefficients $\kappa$ and $\nu$ are constant. This reduces our governing equations to:

$$
\begin{aligned}
& 0=-\frac{\partial p}{\partial \xi}-\rho g \sin \alpha_{0}+\rho_{0} \nu \frac{\partial^{2} v}{\partial \eta^{2}} \\
& 0=-\frac{\partial p}{\partial \eta}-\rho g \cos \alpha_{0}
\end{aligned}
$$

Similarly, the density equation reduces to:

$$
v \frac{\partial \rho}{\partial \xi}=\kappa\left(\frac{\partial^{2} \rho}{\partial \xi^{2}}+\frac{\partial^{2} \rho}{\partial \eta^{2}}\right)
$$

Note that even though the velocity field only varies in the $\eta$ direction, since gravity is not aligned with the direction of variation, this remains technically a two-dimensional problem, so two momentum equations are required. Our boundary conditions are that the velocity must go to zero at the bottom (no-slip), the slope-normal density gradient must be zero at the bottom boundary, and that the along-slope velocity and density perturbations induced by the boundary must decay to zero as you move far from the boundary:

$$
\begin{array}{r}
v, \frac{\partial \rho}{\partial \eta}=0 \quad, \quad \text { at } \eta=0 \\
v \rightarrow 0 \quad, \quad \text { as } \eta \rightarrow \infty \\
\rho \rightarrow-\frac{N^{2} \rho_{0} z}{g}, \quad \text { as } \eta \rightarrow \infty
\end{array}
$$


Phillips assumes a solution for the density field that has the form:

$$
\rho=\rho_{0}\left(1-\frac{N^{2}}{g}\left(\xi \sin \alpha_{0}+\eta \cos \alpha_{0}\right)+r(\eta)\right)
$$

This density field is split into an average density, $\rho_{0}$, a background stratification given in the second term and having buoyancy frequency $N$, and a perturbation from that background stratification $\rho_{0} r(\eta)$ induced by the boundary layer.

By cross-differentiating equations (1.2) and (1.3), and then substituting (1.8) into the result and combining it with (1.4), Phillips obtained a single fourth-order, constant-coefficient ordinary differential equation equation for the density perturbation:

$$
r^{\prime \prime \prime \prime}(\eta)+4 \gamma_{P}^{4} r(\eta)=0
$$

The solution is for the density and velocity fields is:

$$
\begin{aligned}
\rho & =\rho_{0}\left(1-\frac{N^{2}}{g}\left(\xi \sin \alpha_{0}+\eta \cos \alpha_{0}\right)-\frac{N^{2} \cos \alpha}{\gamma_{P} g} e^{-\gamma_{P} \eta} \cos \left(\gamma_{P} \eta\right)\right) \\
v(\eta) & =2 \kappa \gamma_{P} \cot \alpha_{0} e^{-\gamma_{P} \eta} \sin \left(\gamma_{P} \eta\right)
\end{aligned}
$$

In all of this, $\gamma_{P}$ is the reciprocal boundary layer thickness $\gamma=\frac{1}{\delta}$ :

$$
\gamma_{P}=\left(\frac{N^{2} \sin ^{2} \alpha}{4 \nu \kappa}\right)^{\frac{1}{4}}
$$

The resulting velocity field, shown in Figure 1-7, features a strong up-slope bottom current with a small down-slope flow above it. The down-slope component has less than $5 \%$ the volume flux of the primary up-slope current. There are a couple of features of this solution that are worth remarking on. Higher rates of mixing $\nu$ and $\kappa$ are associated with thicker boundary layers. Shallower slopes are are also associated with thicker boundary layers. 


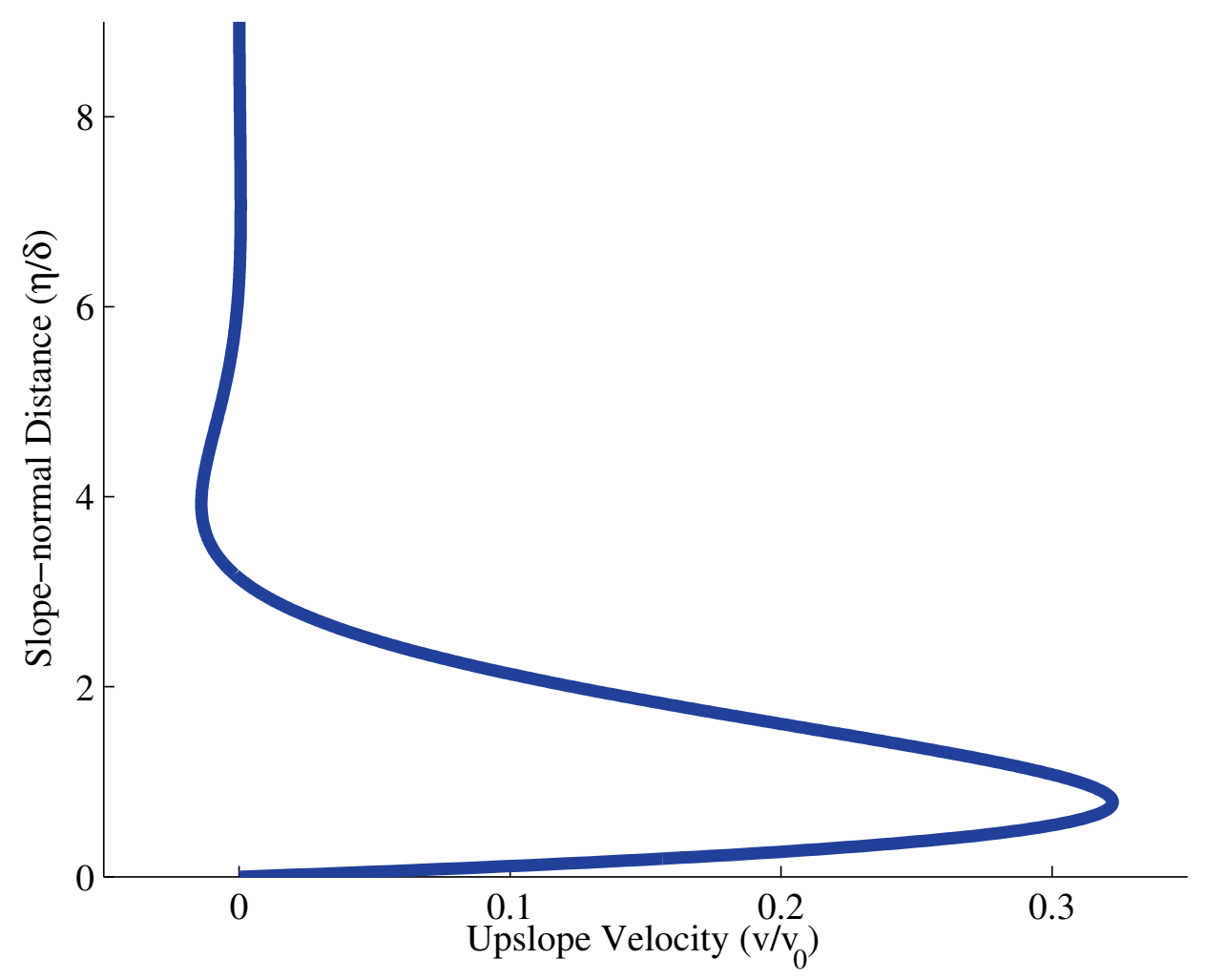

Figure 1-7: The upslope velocity for a boundary layer of the type Phillips [1970] described. It is a bottom-intensified upslope current. This is taken from equation (1.11).

Most interestingly, if we integrate the boundary layer velocity over $0<\eta<\infty$, we find:

$$
Q=\int_{0}^{\infty} v \mathrm{~d} \eta=\kappa \cot \alpha_{0}
$$

The total volume flux $Q$ is independent of the viscosity and the stratification. It depends only on the diffusivity and the bottom angle. At first, this seems very odd. However, if we return to our conceptual picture of a downward heat flux accumulating at the boundary and generating a horizontal density gradient, this makes more sense. The rate of heat flux is proportional to $\kappa$ and the horizontal gradient is proportional to $\alpha_{0}$. In fact, Thorpe [1987] showed by directly integrating the density equation that if $\kappa$ varies with its distance from the bottom, the volume flux constraint is set by the far-field value $\kappa_{\infty}$, not the local value. 


\section{2 .2 Subsequent Studies of Diffusive Boundary Layers}

Building on the ideas of Phillips [1970] and Wunsch [1970], many other authors have contributed to elaborating the dynamics of diffusive boundary layers on sloping bottoms, using theory, numerics, observations, and laboratory experiments.

There have been a couple of interesting laboratory tests of the diffusive boundary layer. Peacock et al. [2004] were able to confirm quantitatively that the velocity in the boundary layer has the predicted relationship with bottom slope $\alpha_{0}$ from equation (1.11). Phillips et al. [1986] explored the effect of a bottom-intensified diffusivity by doing an experiment with a sloping bottom covered by an agitated mat that stirred up a layer of turbulence. Unfortunately, they did these experiments with a two-layer stratification, not a continuous stratification. Nonetheless, they were able to observe an upslope flow close to the boundary and a downslope flow above it, as is predicted by a diffusivity that decreases with height. The downslope flow dramatically reduces the total up-slope transport, but in increases the vertical shear, so may contribute to the strength of the turbulence in the boundary layer. St Laurent, et al., (2001a) predict a similar flow pattern in the $22^{\circ} \mathrm{S}$ canyon using microstructure and hydrographic data collected in the BBTRE. However, their predicted velocities are an order of magnitude less than those measured by Toole's [2007] current meter, so it is unclear whether this reflects a real effect or a coincidence.

Further building on Phillips and Wunsch, Thorpe [1987] developed an analytical solution for the steady boundary layer with rotation. His solution shared many characteristics in common with an Ekman layer. However, as I discuss in Chapter 3, he found that unlike the Ekman layer, the along-isobath velocity in the far-field is not arbitrary. Rather, it is

set by the boundary, and there is one, and only one, far-field velocity that allows a steady state boundary layer. Drawing on both Thorpe's solution and Phillips's experiments, Garrett [1990] came to the conclusion that boundary mixing is probably not significant for the ocean, because the boundary layer quickly becomes homogenized, so additional mixing does not make any difference. He and others had made the same argument earlier in response to Armi's work on boundary mixing, discussed above. Though not directly a part 
of this literature, it should be noted that observations do not bear out the hypothesis of a homogenized bottom boundary layer above sloping topography (see, for example, Figure 1-5 above or Kunze et al., 2002).

A larger literature on boundary layers over sloping bottoms deals with so-called 'Ekman layer arrest.' This literature does not explicitly adopt the framework of Phillips and Wunsch, but instead looks at Ekman transport on slopes. Up- or downslope transport is opposed by bouyancy forces, which can inhibit Ekman transport and prevent steady solutions from being established. There has been a substantial focus on the asymmetry between upwelling-favorable and downwelling-favorable Ekman transport, and the resulting asymmetry in the dynamics of the bottom mixed layer. Based on numerical simulations, Condie [1999] suggested that downwelling Ekman velocities might lead to greatly enhanced mixing efficiencies, so boundary layers might be able to convert a large portion of dissipated kinetic energy into potential energy by mixing. Trowbridge and Lentz [1991] observed this asymmetry on the California coast.

An important contribution came from MacCready and Rhines [1991] and Garrett et al. [1993] who were able to show that the work on boundary mixing and the work on Ekman arrest were actually two cases of the same problem. The arrested Ekman layer was a transient state that the boundary layer went through on its way to the steady state solution proposed by Thorpe. More recent work has looked at the effect of these boundary layers on the potential vorticity field [Benthuysen and Thomas, 2012] and at the boundary layer response to time-varying forcing [Umlauf and Burchard, 2011].

This entire literature concentrates exclusively on uniform constant slopes with infinite extent. To my knowledge, only two studies address any aspect of diffusive boundary layers over non-constant slopes. Woods [1991] identified a purely advective mechanism by which the bottom boundary layer can affect the stratification. Using equation (1.13) and conservation of volume, he deduced that there must be an equal volume transport downward in the interior of the basin, giving a widely distributed downwelling velocity. This volume flux in the boundary layer is independent of the size of the basin, and the horizontal area of 
a basin with sloping walls varies with depth, so the downwelling velocity also varies with depth. This can increase the stratification at some depths and reduce it at others. This advective modification of the stratification is seen clearly in experiments (Peacock, personal communication, 2009). Cummins and Foreman (1998) modeled unforced flow over an isolated seamount for both constant mixing coefficients and bottom-intensified mixing. They observe a diffusive boundary layer, and the deflection of isopycnals to intersect the slope at a right angle, but their simulations were dominated by the geostrophically balanced azimuthal (along-isobath) current that came from the deflection of isopycnals. They modeled a few different profiles of mixing coefficients, and found that the azimuthal currents had robustly similar characteristics. Unfortunately, little attention was given to the diffusive boundary layer itself.

In sum, a number of interesting studies have been done on diffusive boundary layers, but they have generally focused on one-dimensional topography-infinite, flat constant slopes. Based on this literature, several authors have made the argument that boundary mixing may be less important because the boundary layer becomes rapidly homogenized, after which additional turbulent dissipation makes no dynamical difference. At the same time there has been a growing recognition throughout the physical oceanography community that topography is playing a key role in abyssal mixing. And clearly the bottom of the ocean looks nothing like an infinite, flat constant slope. My goal in this thesis is to start to bring these two sets of ideas together.

\subsection{Structure of This Work}

Given the huge role of topography suggested by observations of abyssal mixing and especially in abyssal canyons, and given the substantial study of stratified fluid boundary layers over constant slopes, this thesis has two major goals:

1. to better understand the basic physics of how stratified fluids interact with spatially varying boundaries; 
2. to apply that knowledge to better understand how ocean-bottom topography affects the mixing, stratification, and transport of the ocean, especially in the context of abyssal canyons.

I approach these goals by beginning with the known solution for a uniform bottom slope, and gradually build complexity. I use theory and idealized process modeling to maintain grasp of basic physics as complexity increases. Ocean bottom topography varies on all scales, so I choose to focus on the scale of the individual canyon and the individual sill within that canyon. That is, on the scale of $1-100 \mathrm{~km}$ in the horizontal and $10-1000 \mathrm{~m}$ in the vertical.

This thesis has three substantial parts. Chapter 2 looks at boundary layers over nonrotating but varying topography. This is an inherently two-dimensional problem, and I cover a number of simple types of topography: changes in bottom slope, isolate sills, and corrugations on sloping bottoms. I find that there are a number of different boundary layer geometries that can arise for a single type of topography, and that the boundary layer behavior over a particular topography can be accurately predicted a priori. These various behaviors suggest mechanisms for both enhancing mixing in the boundary layer and exchanging mixed fluid more efficiently with the far field.

In Chapter 3, I extend the non-rotating work to cover two-dimensional rotating topography. The most interesting result from this section is that at steady state, the rotating (Ekman-like) boundary layer behavior responds to the average slope, while the diffusive (Phillips-like) boundary layer behavior responds to the local slope. As a result, rotation suppresses exchange between the boundary layer and the far field. The rotating and buoyant dynamics also mean that the two effects sometimes cancel each other and sometimes reinforce each other, even though the system is ultimately driven by buoyant effects.

In Chapter 4, we look at three-dimensional dynamics in canyon-like, but still idealized, domains. In this case, the bottom boundary layer continues to look much like a Phillips layer, but it forces an outer boundary layer with geostrophic recirculations that fill the canyon bottom. As in the two-dimensional case, introducing varying topography provides 
a mechanism for enhancing mixing in the boundary layer and exchange with the far field.

In the final chapter, I summarize the contributions of this work, as well as its limitations, and discuss some avenues for future research. 


\section{Chapter 2}

\section{Diffusion-Driven Boundary Layers over Varying Topography, without Rotation}

\subsection{Introduction}

We begin with a discussion of the behavior of diffusive boundary layers over non-rotating, spatially varying topography. As discussed in the introduction, for all the work that has been done on diffusive boundary layers, the subject of varying topography remains unstudied. In effect, this has limited the problem to one dimension of variation, in the slopenormal direction. My goal in this chapter is to extend the discussion to a variety of simple topographies, like isolated changes in slope, isolated sills, and sinusoidal corrugations on slopes. Even these simple topographies introduce a variety of interesting dynamics, as we shall see in what follows.

This work was inspired by the study of deep ocean canyons. However, even though rotation plays an important role in most open-ocean processes, I will begin with a study of non-rotating boundary layers. This is for a few reasons. First, it's simpler, and it's often very difficult to understand the more complicated problem without first getting some handle on the simpler one. Second, since the canyons are narrow compared to the barotropic and first baroclinic Rossby radius, geostrophic eddies are largely excluded. It should be noted, 
however, that the limit for truly non-rotating dynamics arises when the canyon is narrower that the Ekman layer thickness $\sqrt{\nu / f}$, which is obviously not the case in the real ocean. Thirdly, as we will discuss in the next chapter, both Wunsch [1970] and Thorpe [1987] find that the upslope flow has an identical structure with and without rotation (though both assumed no variations in topography), so the non-rotating problem has many similarities to the two-dimensional rotating problem.

Unfortunately, the observations of Atlantic ridge-flank canyons do not allow us to determine conclusively how strong the effect of rotation is, as they offer very little cross-canyon information. For the time being, we will neglect it, though the importance of rotation will be taken up in much greater detail in the following chapters.

Even without rotation, a variety of interesting dynamics arise when varying topography is introduced. Many of these dynamics are forced by variations in the volume flux carried by the boundary current. As previously demonstrated, the boundary layer volume flux over a uniform slope is given by:

$$
Q=\kappa_{\infty} \cot \alpha
$$

$\kappa_{\infty}$ is the diffusivity in the far field, and $\alpha$ is the slope angle. Shallower slopes carry more fluid, so increasing the slope forces fluid out of the boundary layer and into the far field. Conversely, decreasing the slope entrains fluid from the far field into the boundary layer. These exchange flows with the far field can carry the same order volume flux as the primary boundary layer current, and can dramatically affect the stratification both close to the topography and in the far field by forcing out the well-mixed fluid in the boundary layer. In my Masters thesis, I demonstrated that high-resolution numerical models of boundary layers over broken slopes showed the same local volume and mass flux as given in equation (2.1) when the slope of the downstream region $\alpha_{1}$ is less than the slope of the upstream region $\alpha_{2}$ [Dell, 2010]. An example of the dynamics of a boundary layer over a broken slope is shown in Figure 2-1, where the excess boundary layer flux is forced into the far field in an intrusion current at the level of the change in slope. The density of the fluid in the intrusion is bounded by the relatively narrow range of densities spanned by the boundary 


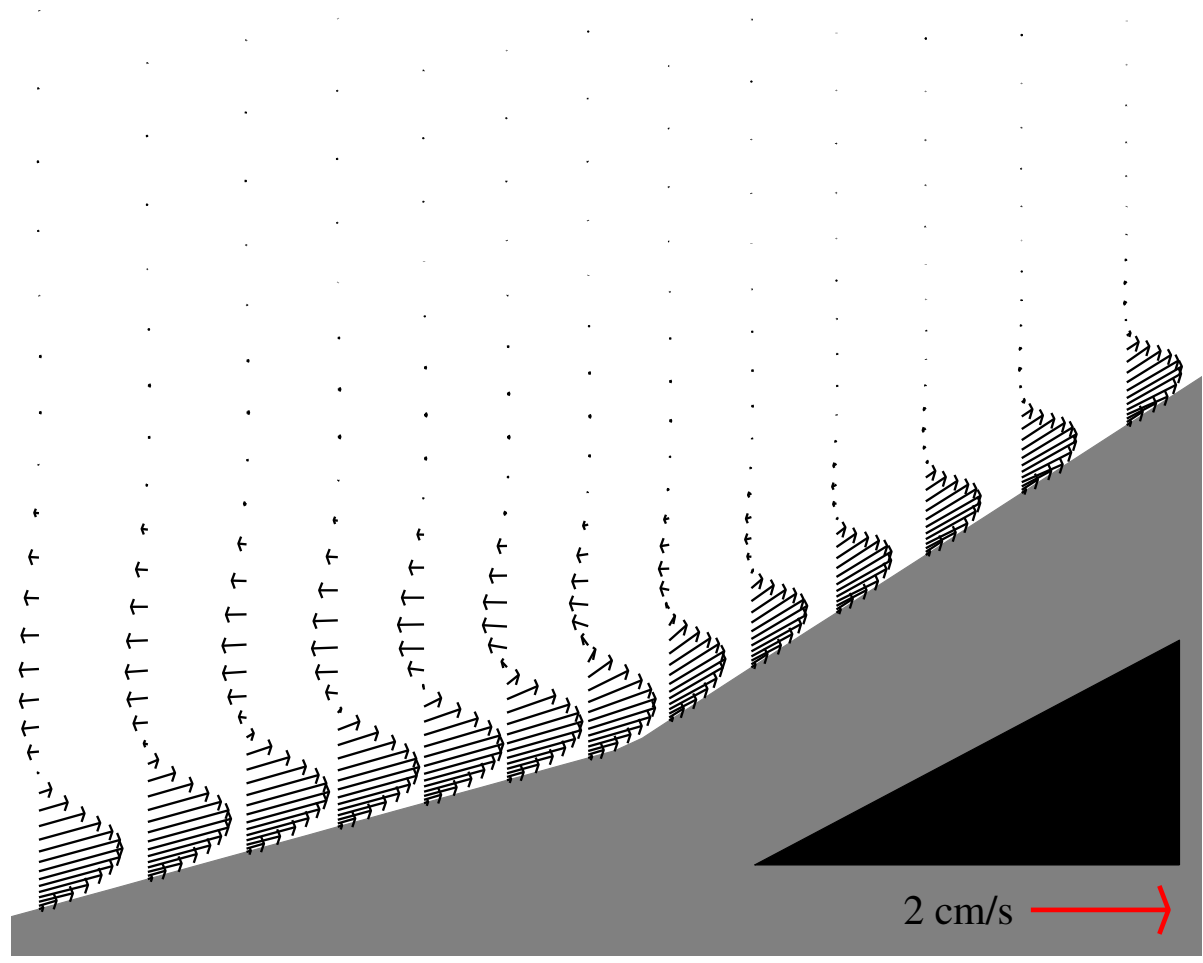

Figure 2-1: Changes in the bottom slope require fluid exchange between the boundary layer and the far-field. The above $y z$ velocity section shows the larger velocities over the shallower slope to the left of the change in slope angle, and the counterflowing intrusion flowing left into the far field from the change in slope angle. In the oceanographic parameter range, these intrusions can carry similar volume and mass fluxes to the boundary layer current. As with all such figures in this chapter, the red arrow shows the size of $2 \mathrm{~cm} \mathrm{~s}^{-1}$, and the black triangle is $50 \mathrm{~m}$ tall and $1000 \mathrm{~m}$ wide.

layer.

These kinds of exchange flows are crucial to the general importance of boundary layer mixing to the far field fluid. Previous authors have suggested that boundary mixing may have limited effect on the oceanic abyssal stratification, as it may simply stir the already homogeneous fluid confined to the boundary region (see for example Ivey, 1987). However, the dynamics discussed in this chapter provide a robust mechanism by which the mixed fluid in the boundary layer can be returned to the far field, and new, stratified fluid brought into the boundary region for transformation. As we will see over the course of this chapter, though, the interaction between the boundary layer and the far-field fluid is generally not 
as simple as direct inference from equation (2.1) might suggest.

In this chapter, I will discuss the dynamics of that interaction using numerical and theoretical results for broken slopes, slopes with corrugations, and slopes with isolated topography. The numerical methods are detailed in the section on broken slopes; this discussion in applicable to all the numerical results presented.

\subsection{Boundary Layers over Broken Slopes}

The simplest varying topography is an inclined bottom that changes slope at a single point (Figure 2-1). In this section, I extend the work of my Masters thesis to cover all slope angles for both sections of the broken slope.

\section{2 .1 Numerical Results}

For broken slopes, the locally-constant slope assumption gives accurate predictions of the mass and volume exchange between the boundary layer and the far-field using the assumption that the boundary layer behaves nearly everywhere as though the slope were locally constant (Figure 2-2). This type of topography does not introduce any length scales in addition to the boundary layer thickness associated with each of the two constant slopes, so it is the most likely topography to be effectively described by assuming that the constant slope solution holds everywhere in the boundary layer. The flow remains linear everywhere, and the dynamical balance is the same as in the case of a constant slope: between viscous dissipation and the horizontal pressure gradient that results from isopycnals bending downward to intersect the insulating boundary at a right angle. Since the dynamics are linear, this description is equally true in the $\alpha_{1}>\alpha_{2}$ range (convex slopes) as in the concave slopes discussed in my Masters thesis. In the case of a convex slope, fluid is sucked into the boundary layer from the far field. These exchange flows, analogous to the 'secondary circulations' induced by variations in $\kappa$ and $N$ in Phillips et al. [1986] and Garrett [1991] can be as large as the 'primary' flow of the boundary layer current itself. 


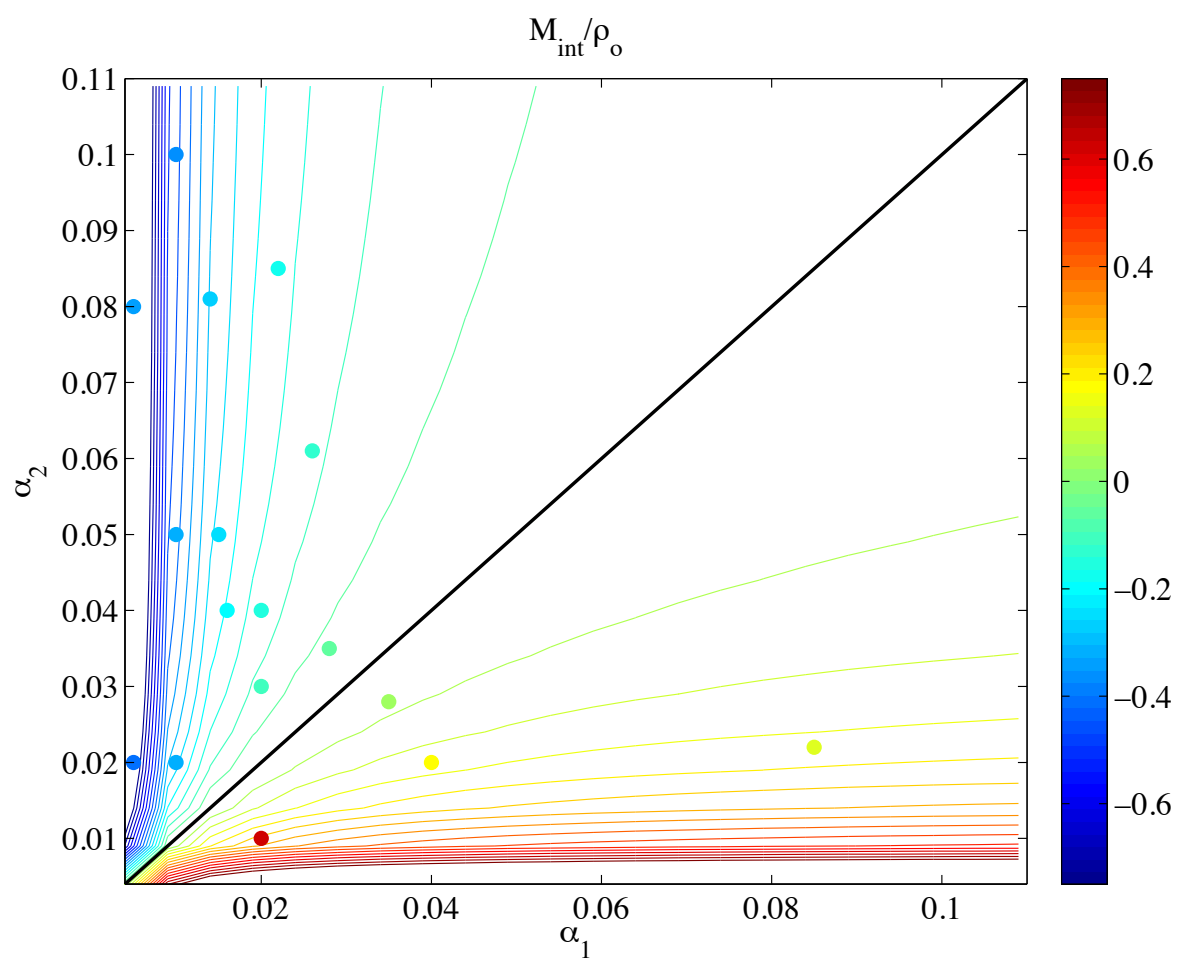

Figure 2-2: The nondimensional mass fluxes in the intrusion or entrainment layers are accurately predicted by assuming that the constant-slope solution is locally true everywhere. The contours show the value predicted by the mass budget in Dell [2010], while the color of the dots show the mass fluxes calculated numerically. Any difference in color between a dot and the adjacent contour is a theory-model mismatch. The mismatches only exceed $2-3 \%$ in cases with the shallowest slopes, and I believe they are a result of computational limitations, as the shallowest slopes require the comparatively long time-scales and large domains to calculate accurately.

Of course, this prediction is limited to the range of slope angles for which the numerics are reliable. Very small angle slopes may not be accurately described by this approach.

\section{2 .2 Numerical Methods}

I performed all of the numerical experiments reported in this chapter using the Regional Ocean Modeling System (ROMS), developed by Shchepetkin and McWilliams [2005]. ROMS is a fully non-linear hydrostatic primitive equation model. It has a free surface, and so calculates the barotropic and baroclinic modes separately. ROMS uses topography- 
following coordinates, also known as $\sigma$-coordinates. It was primarily for this reason that the ROMS was selected for this study, as $\sigma$-coordinates can represent smoothly varying, instead of step-like, topography and so more effectively represent bottom boundary layers.

In the simulations presented in this chapter, there were 60-200 vertical levels, depending on the slope angle and overall water depth in each model run. The vertical resolution increased monotonically on approach to the bottom boundary, with vertical grid spacing varying from about $0.5 \mathrm{~m}$ at the bottom to about $15 \mathrm{~m}$ at the top of the domain. For comparison, the boundary layer scale height $\delta$ in the simulations ranged from $6-40 \mathrm{~m}$. We found that the free surface had a substantial effect even when the water depth was $10 \times \delta$, so all the computations had a minimum depth of at least $35 \times \delta$.

Mixing in the model was represented as an explicitly-specified eddy diffusivity $\kappa$ and eddy viscosity $\nu$. The eddy Prandtl number $\mathbb{P}_{r}=\frac{\nu}{\kappa}$ was assumed to be one. Using eddy mixing coefficients is physically problematic because it presupposes a source of energy to drive turbulent eddies. It is also concerning because the strength of eddy mixing and so the appropriate size of the eddy mixing coefficients in the ocean decline as one moves away from the boundary (cf. Figure 1-2), and this has strong effects on boundary layer dynamics (see Appendix 2.A ). In spite of these concerns, however, using eddy mixing coefficients based on typical $\kappa$ inferred from observations in abyssal canyons provides a good starting point: numerically straight-forward and physically and observationally plausible. The noslip bottom boundary condition was approximated with an artificially enhanced bottom drag, strong enough to bring the velocity to zero over the distance of one grid cell. The results are insensitive to the specific value of the coefficient of drag. No surface stress or external currents were applied.

The simulations in this chapter were all strictly two-dimensional. Two different types of lateral boundary conditions were used. For simulations with no overall bottom slope, I used closed lateral boundaries-vertical walls. The lateral boundaries for simulations with an overall bottom slope posed the largest computational problem. To model a boundary layer with no along-slope variation, we would like to have a domain that is infinitely large in the 
along-slope direction. However, because the bottom boundary has a slope and the water is stratified, it was not possible to use periodic boundary conditions. Ultimately, the lateral boundary conditions that were most successful at reproducing the theoretical solution for a constant slope involved specifying the barotropic momentum at the inflowing boundary and specifying the full, depth-dependent, baroclinic momentum at the outflowing boundary. In order to match the flow to this outgoing boundary condition, a long uninterrupted section of constant slope topography was inserted between the study region and the both boundary. This section was typically $5000 \mathrm{~m}$ or longer, 2-10 times the adjustment length scale $L_{a d j}$ for boundary layer development given by the typical velocity of the boundary layer $U_{B L}$ and the typical timescale for boundary layer development $T_{B L}$ :

$$
L_{a d j} \sim U_{B L} \times T_{B L} \sim \frac{2 \kappa \cot \alpha}{\delta} \times \frac{\delta^{2}}{\kappa} \sim 2 \delta \cot \alpha
$$

For small angles, $T_{B L} \sim \alpha^{-1}$, implying $L_{a d j} \sim \alpha^{-3 / 2}$. As the slope gets shallower, the boundary layer becomes thicker and the adjustment distance becomes longer. In general, the locally constant slope assumption is justified when the topography varies on scales significantly larger than $L_{a d j}$. This is a much more stringent condition than the usual requirement that the radius of curvature of the topography be much larger than the boundary layer thickness $\delta$. In an effort to further reduce the effects of the lateral boundaries, the lateral grid spacing was 'telescoped,' increasing exponentially away from the test section. The lateral grid spacing varied from about $65 \mathrm{~m}$ to over $900 \mathrm{~m}$.

Radiation conditions were used for the free surface and for the active tracers, namely heat and salt. Though the model included both active tracers, only temperature was used for stratification, and salinity was taken as constant throughout the domain. The stratification was not enforced at either boundary, but rather specified as an initial condition. For the parameter regions explored, the boundary layer was able to reach a steady state before any significant changes had occurred in the stratification. There was no variation in the along-isobath direction. 


\subsection{Corrugated Slopes}

Superimposing a sine wave on a constant slope introduces much richer and more interesting dynamics than we saw in the case of the broken slope. With corrugations, there are sills and troughs, the possibility for both slow and rapid changes in slope, slope reversals, and many other potential sources of complexity. In this section, I explore the range of behaviors the boundary layer produces in the range of topography near that observed in mid-ocean ridge canyons, and some of their potential implications for the dynamics of the full water column. The range of topographies covered in these numerical experiments is summarized in Table 2.1.

\begin{tabular}{l|rccc} 
Background Slope & 0.012 & - & 0.08 & \\
Sill Height & 7.6 & - & 158 & $\mathrm{~m}$ \\
Sill Separation & 0.3 & - & 24.5 & $\mathrm{~km}$
\end{tabular}

Table 2.1: The range of parameters used to specify slopes and the size of corrugations or sills in the model runs in this section. Most canyon sills in the ocean are in this parameter range, but the largest sills are not. However, these ranges allowed us to fully explore the parameter space of relevant non-dimensional numbers, as shown in Figure 2-13.

\section{3 .1 Description of Numerical Results}

Corrugations have areas of both increasing and decreasing slope, leading to both fluid intrusions from the boundary layer and entrainment into the boundary layer at different points (Figure 2-3). In this first example, the small-amplitude variations of the bottom slope lead to a stack of alternating exchange flows between the boundary layer and the far field. Fluid is forced out of the boundary layer where the slope is increasing, and sucked into the boundary layer where the slope is decreasing. This has a couple of interesting consequences. First, it can change the stratification far from the boundary layer by selectively adding or removing fluid of a given density from the far-field (Figure 2-4). This will either sharpen or weaken stratification in the far field at the level of topographic slope decreases or increases. In addition, it can create substantial vertical velocity shear even in the far 


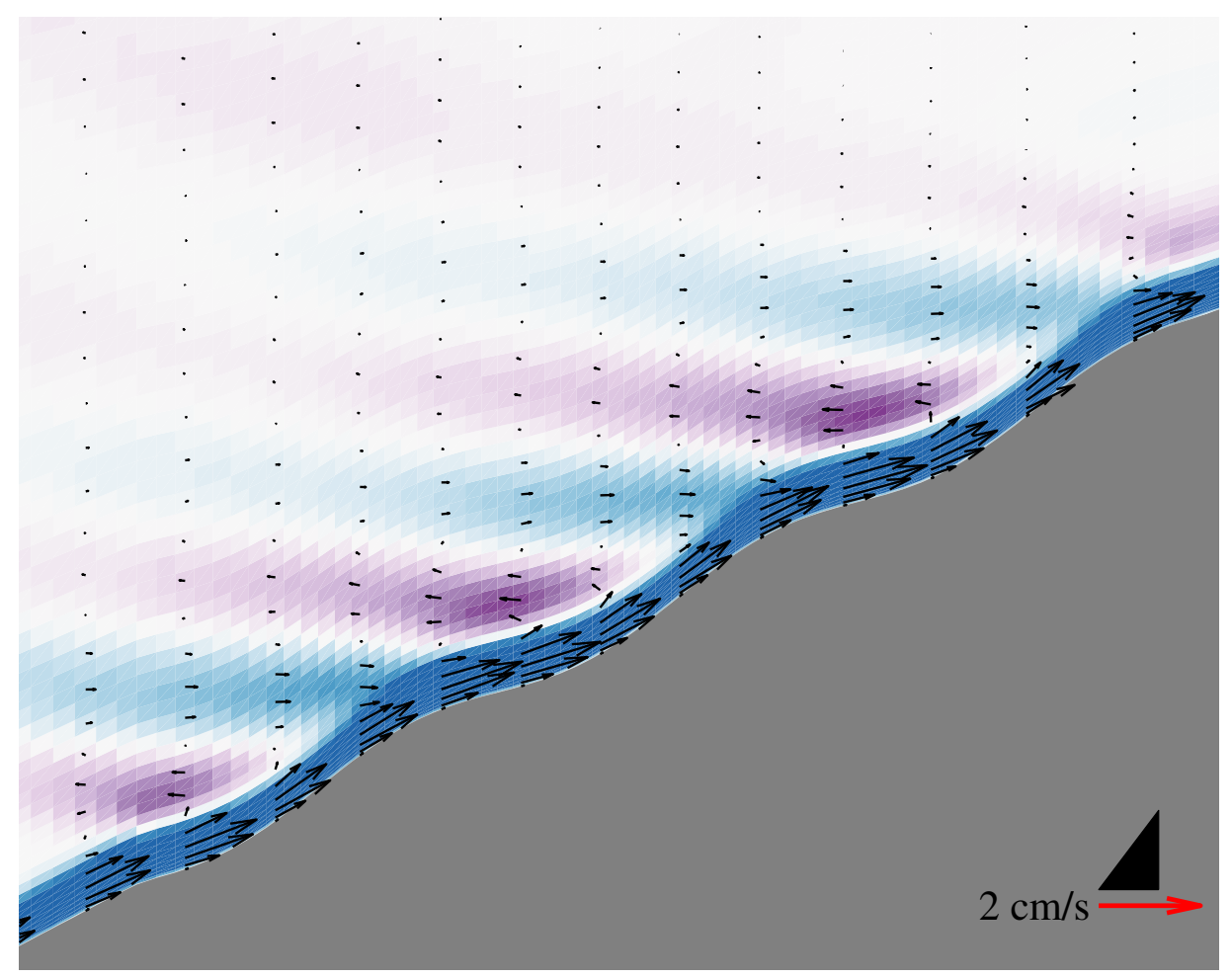

Figure 2-3: The velocity field induced by a corrugated sloping bottom in small amplitude (linear) parameter range. In addition to the arrows, the color shows the horizontal velocity. Substantial amounts of fluid are exchanged between the boundary layer and the far field through a vertical stack of jets flowing into and out of the boundary layer. This affects the stratification by selectively adding or removing fluid of given densities. It also creates vertical shear. The black triangle is $50 \mathrm{~m}$ tall and $1 \mathrm{~km}$ wide.

field.

As we vary the height of the corrugations, we observed three distinct types of flow behavior for unforced laminar flow over a corrugated sloping bottom, with each type of behavior corresponding to a distinct parameter range. For small amplitude bumps, where the boundary has no sections of negative slope, a vertical stack of horizontal or nearlyhorizontal jets is formed, like in Figure 2-3. The flow remains linear, with advection negligible compared to pressure and viscosity (Figure 2-5). For very large amplitude bumps, an up-slope boundary layer can develop on the reverse side of the sill, as in Figure 2-9. These 


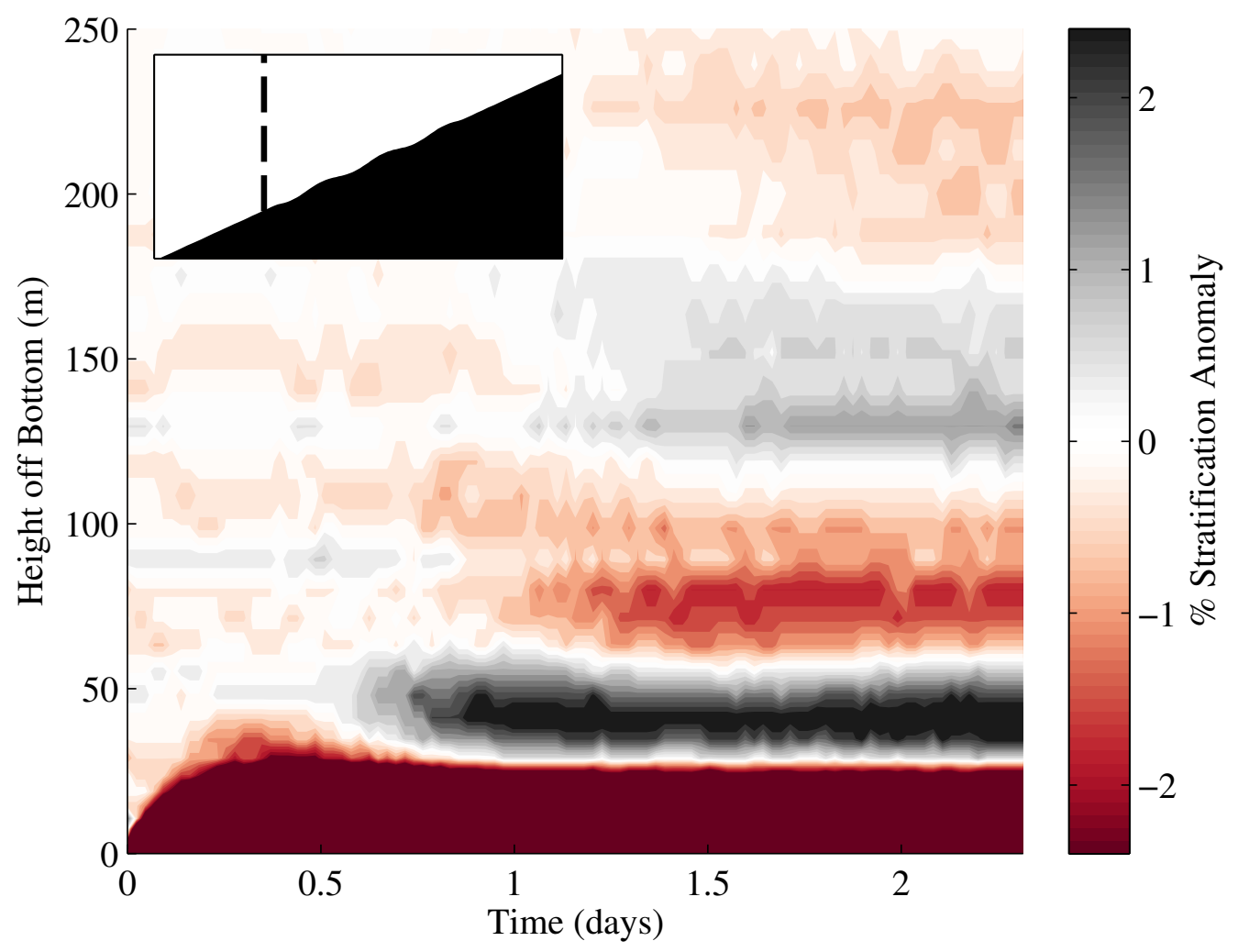

Figure 2-4: A corrugated boundary can cause significant anomalies in the density field far from the boundary. This is a hovmöller diagram of the time evolution of the stratification anomaly in the column indicated in the inset. Stratification strengthened at some depths, weakened at others as fluid is drawn into or forced out of the boundary layer. It is not clear that this will come to a steady state. It is in the linear parameter range.

boundary layers on the back of the sill carry much less fluid than the boundary layers on the front of the sill, even though we would predict that they would carry more under the locally-constant slope assumption discussed in the previous section. However, the fluid again remains largely linear (Figure 2-6), with no significant advection.

The linear dynamics of the very small and very large topography both make intuitive sense. In the small-amplitude limit, we approach the constant slope case, where advection is exactly zero. In the large-amplitude limit, the scale of the boundary layer is much smaller than the scale of the topography, so the boundary layer only feels the local topography. The boundary layer then behaves as if it were on a constant slope with the local slope angle.

As is usually the case in fluid dynamics, the most interesting dynamics happen between 
the two extremes of small and large corrugation amplitude. There we see a range of behavior. Even very small increases in the corrugation amplitude can cause the solution given by the locally-constant slope assumption to break down (Figure 2-7). Most interestingly, as the amplitude is increased somewhat more, we see overflows like the velocity section shown in Figure 2-8. The boundary layer accelerates at each sill, with the largest up-slope velocities found immediately downstream of the sill. In these cases, the horizontal and vertical advection are of the same order as the bottom boundary-condition-driven pressure gradient and the viscous dissipation in the neighborhood of the sill (Figure 2-10).

These different flow behaviors have associated effects on the stratification, both close to the topography and it the far field. Counterintuitively, smaller amplitude topography can have a larger impact on the far-field stratification, because the fluid entering or leaving the boundary layer can propagate away from the boundary. When the corrugations are larger, the fluid can become trapped into partially or completely closed recirculations within the trough between the corrugations, as seen in Figure 2-9. Over time periods much longer than the adjustment timescale of the boundary layer, these troughs become filled with unstratified fluid (Figure 2-11). If there is roughness everywhere along the boundary, the blob of low stratification that is formed might be observed as an extended region of low stratification outside the diffusive boundary layer. This is particularly interesting, as observations of abyssal canyons show that the boundary layer thickness-the thickness over which the stratification is notably reduced and boundary currents can be measured-generally coincides with the height of the highest topographic sills (see St. Laurent et al., 2001b or Thurnherr and Speer, 2003). In many observations, the bottom boundary layer is often as much as $500 \mathrm{~m}$, larger than can be explained by a traditional Phillips/Wunsch boundary layer. We still do not have a good explanation of why these bottom boundary layers are so thick, but strong interactions with topography, of the type discussed here, may help explain these observations. A more detailed discussion of how corrugations might lead to a layer of reduced stratification that extends beyond the thickness of the Phillips/Wunsch layer is presented in Appendix 2.B to this chapter. 


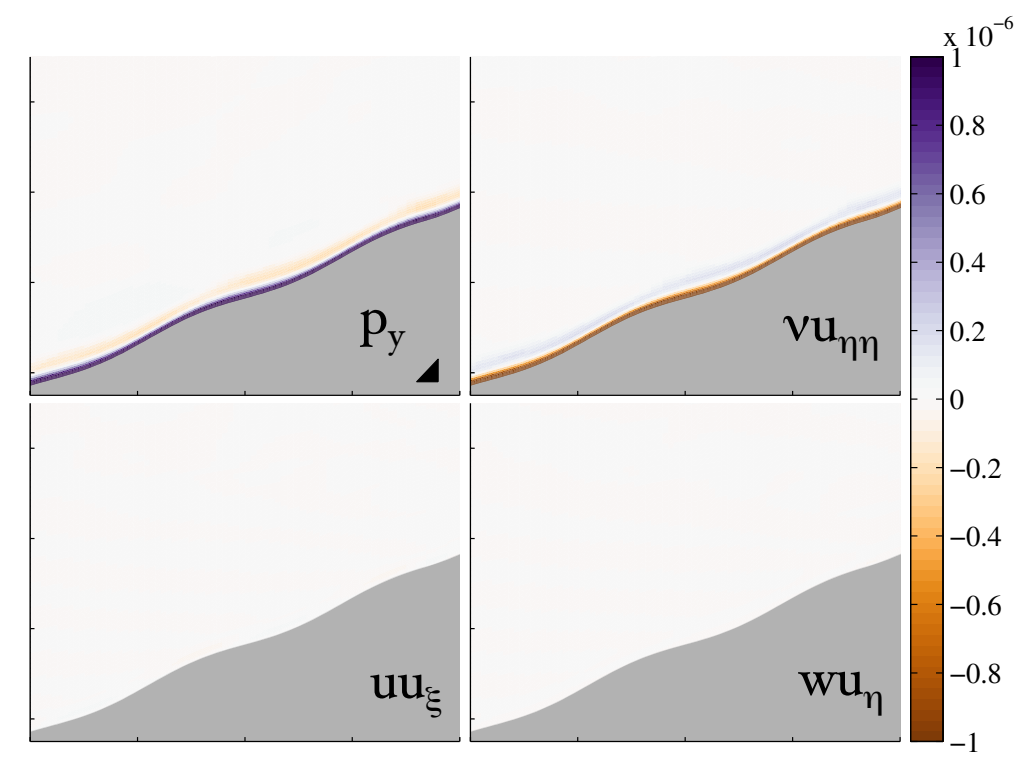

Figure 2-5: For small-amplitude topography, the dynamics remain substantively linear, with the pressure gradient balancing the viscous dissipation in the two upper panels. Advection is negligible here, as you can see from the two lower panels. This case is approaching the constant-slope case where advection is exactly zero.

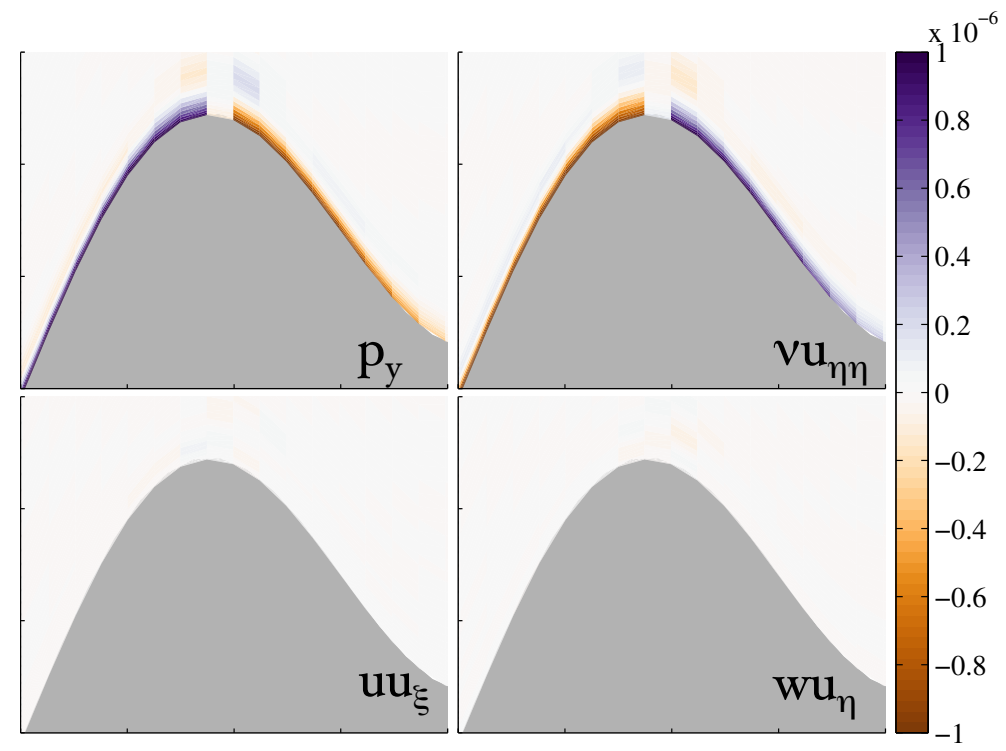

Figure 2-6: For very large amplitude topography, the dynamics also remain substantively linear, with the pressure gradient balancing the viscous dissipation in the two upper panels. Advection is negligible here, as you can see from the two lower panels. These figures are zoomed in on a single corrugation sill to allow the reader to see the boundary layer. 


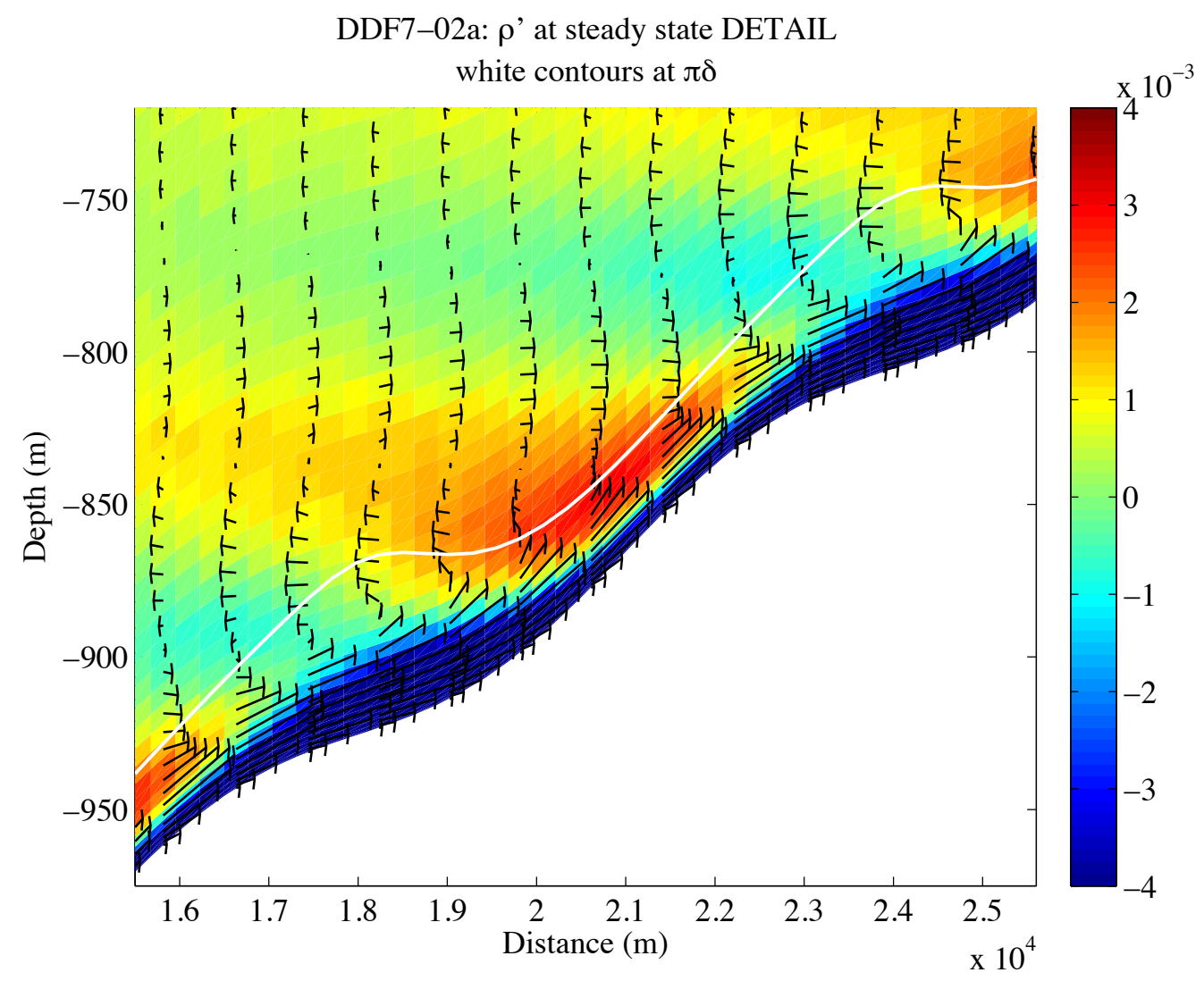

Figure 2-7: Even very small amplitude corrugations can cause dynamics that are substantially different than those predicted by assuming that the slope is locally constant. Here we see the density anomaly $\rho^{\prime}$ with the velocity field superimposed as arrows. The white line is the predicted boundary layer thickness under the locally-constant slope assumption. The actual boundary layer thickness only coincides with the white line on the steepest part of the slope. Associated with this change in boundary layer thickness is a dramatic change in boundary layer flux. The constant-slope assumption breaks down because the wavelength of the corrugations is less than the boundary layer adjustment length scale given in equation (2.2). 


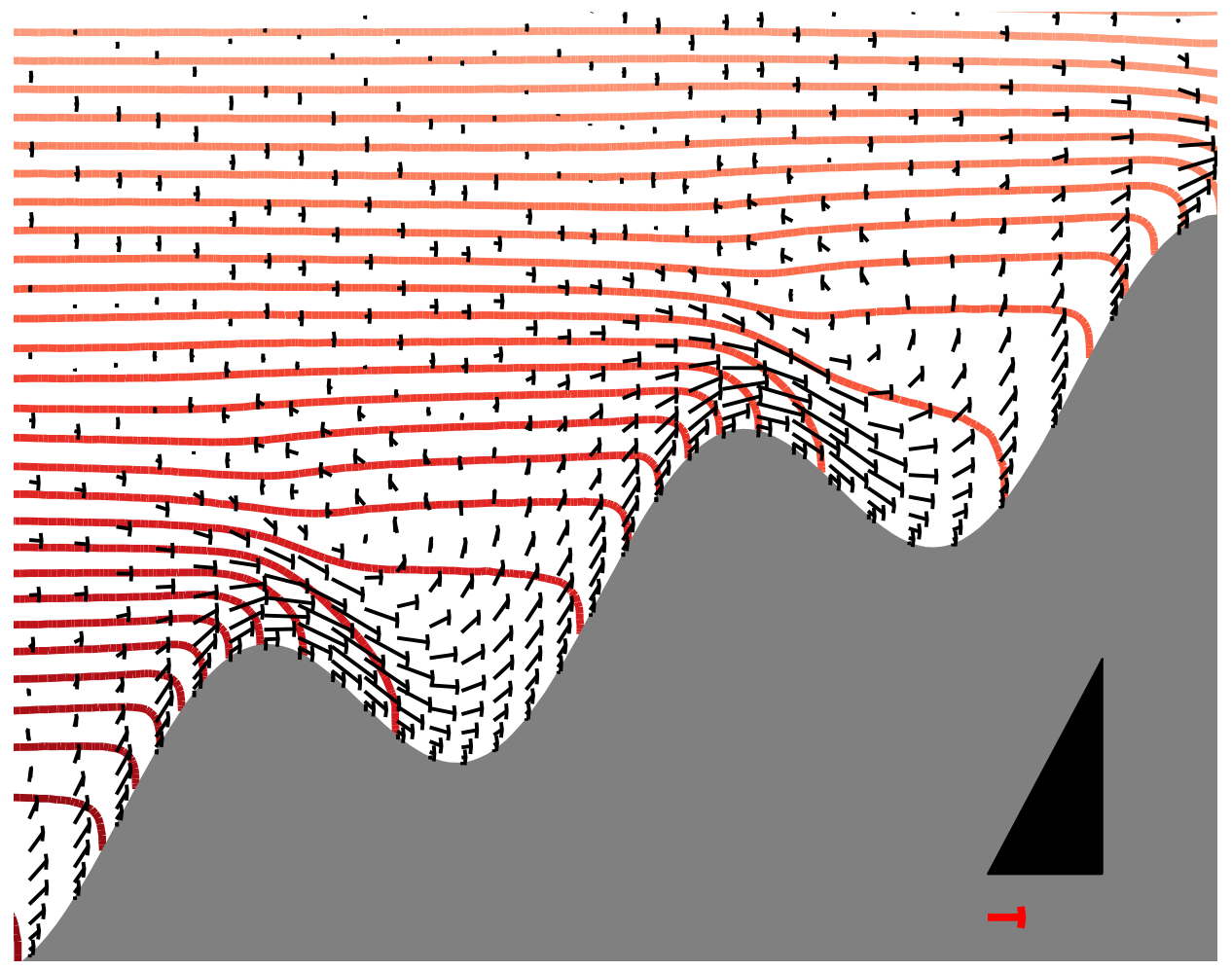

Figure 2-8: In certain parameter ranges, the boundary layer flows can become strongly nonlinear. We see this here in velocity vectors and red isopycnals. There is plunging, overflows, and inertial behavior around sills, with currents dramatically accelerated relative to the constant-slope solution for the mean slope. The maximum along-slope velocityaround $3 \mathrm{~cm} / \mathrm{s}$ in this case-is just downstream of the sill, where the constant-slope solution would predict flow in the opposite direction (from right to left in this figure). These spilling flows carry substantial fluid volume into the trough, and can erode the stratification of the fluid below sill height (Figure 2-11). Here we can see that the isopycnals are dramatically spread in the troughs between the sills. 


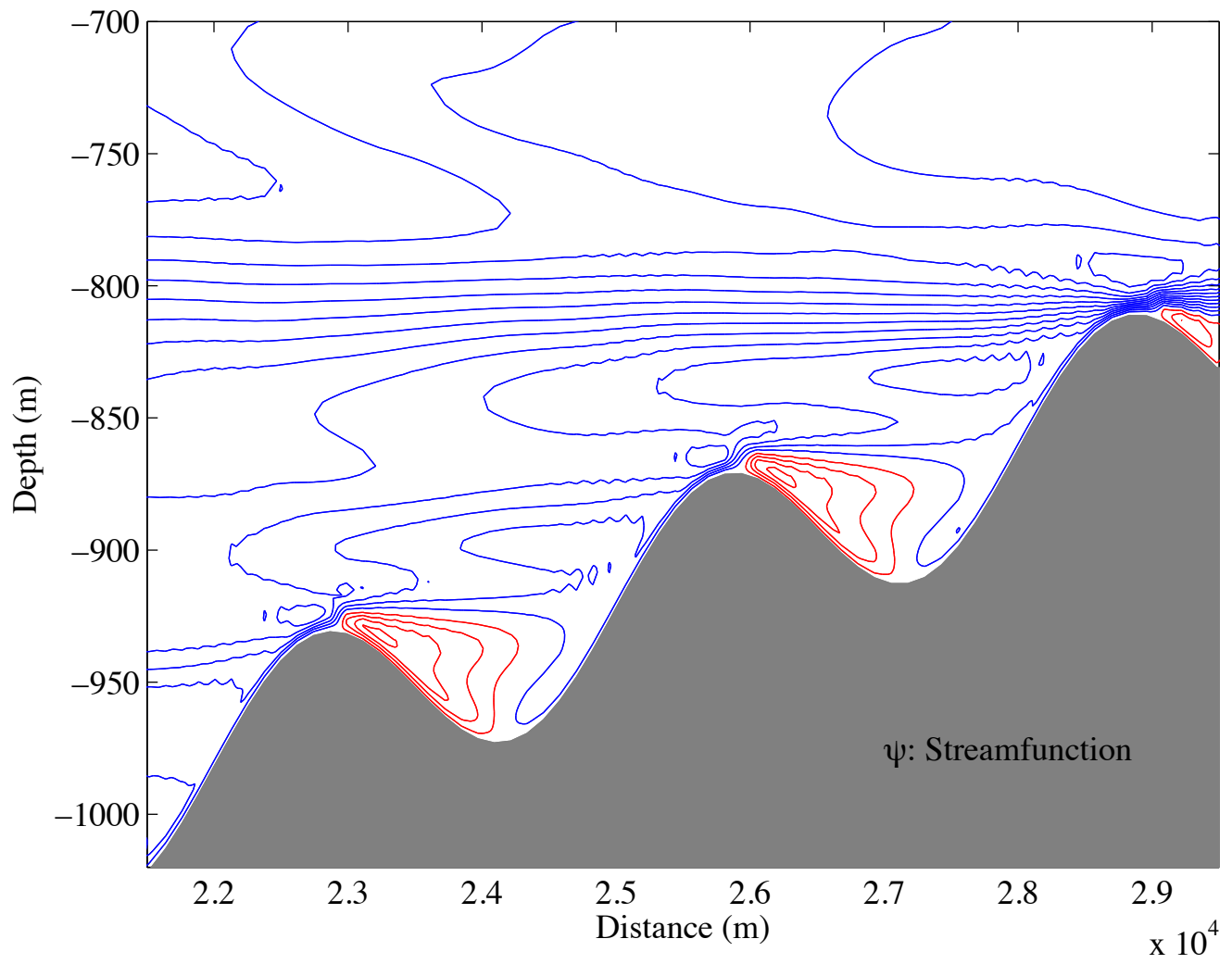

Figure 2-9: When the corrugations are much taller than the boundary layer thickness, local boundary layers can form on the reverse face of each corrugation, as seen here in the streamfunction. This sets up the potential for a conflict between the prevailing upslope flow and the local boundary layer. Blue streamlines are positive (anticlockwise velocity), and red streamlines are negative (clockwise velocity). Intrusions form on both the upslope and downslope sides of each sill. The downslope intrusion can propagate away from the sill, affecting the far-field stratification. The upslope intrusion is confined to the trough between the sills, so the trough can eventually fill up with unstratified fluid of the density at the sill height, even though there is no spilling overflow in this case. 


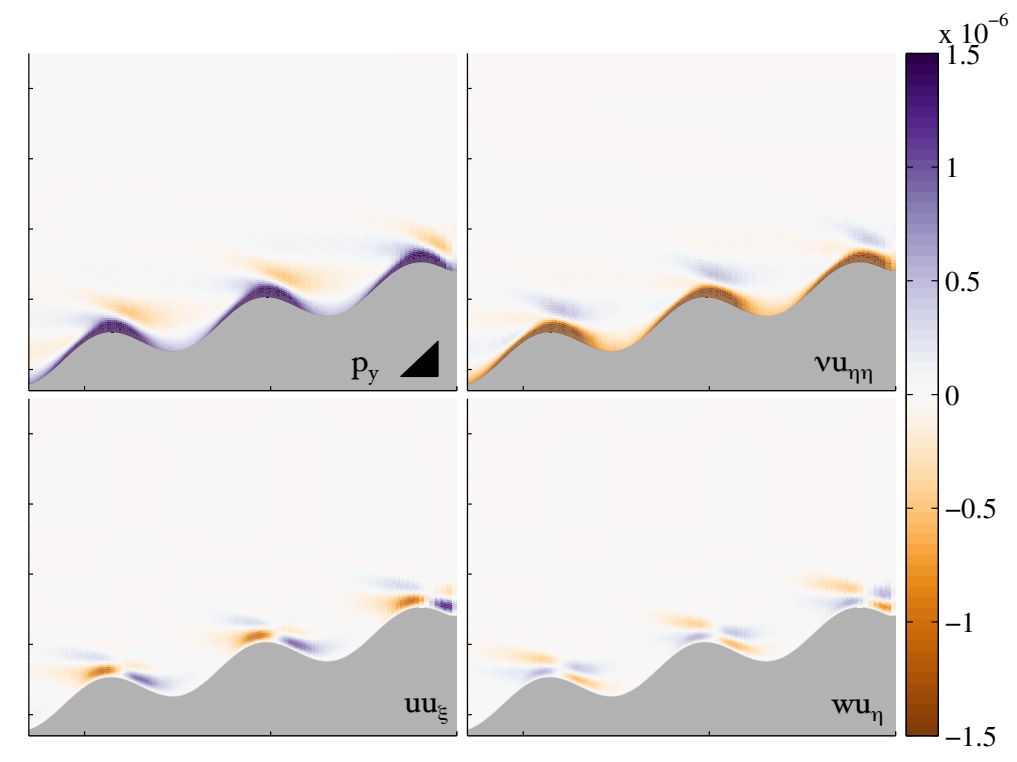

Figure 2-10: For topography with plunging overflows, like that shown in Figure 2-8, the primary dynamical balance remains between the pressure gradient arising from the no-flux bottom boundary condition and viscosity. However, both horizontal and vertical advection are first-order compared with pressure and viscosity. The black triangle is $50 \mathrm{~m}$ tall and $1 \mathrm{~km}$ wide.

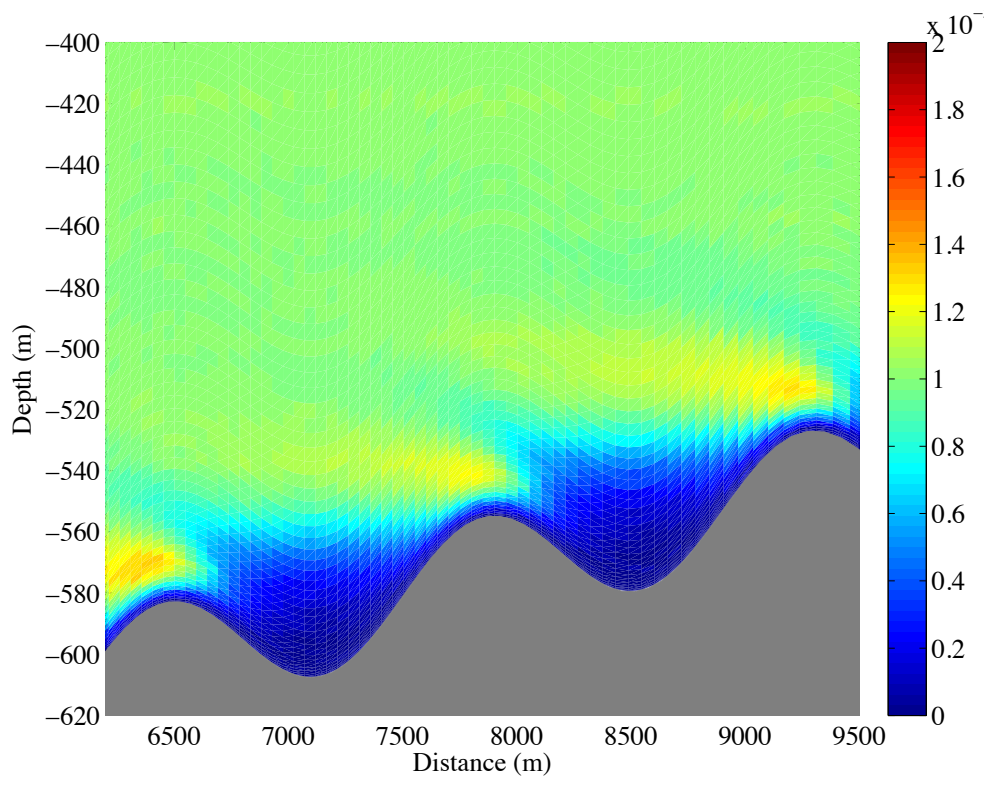

Figure 2-11: Troughs between sills fill up with fluid at the density of the sill height. Over time, the stratification in the troughs is eroded. This may contribute to the phenomenon we observe in ocean-bottom canyons, that the thickness of the weakly stratified boundary layer is approximately the same as the height of the topographic bumps. 


\section{3 .2 Dependence of Flow Geometry on Topography}

These numerical experiments demonstrate that corrugations on slopes can lead to a variety of different boundary layer dynamics and geometries. These different geometries have very different likely consequences for the level of turbulence and mixing in the boundary region and its effect on the background stratification. The three basic flow geometries we observed are:

Linear These boundary layers behave everywhere like the Phillips constant-slope boundary layer. They have no significant advection. They are most effective at exchanging fluid between the boundary region and the far field.

Reversing These boundary layers have upslope flow on both faces of each sill as the constant-slope solution would suggest. This means the boundary layer current reverses direction at each sill and again in each trough. These boundary layers are very effective at homogenizing the fluid in the trough between the sills, so may help to explain why the observed boundary layer thickness in the ocean coincides with the height of the sills.

Overflow These boundary layers have strong advection and acceleration at the sills, and will likely cause greater enhancement of turbulence in the boundary region.

These behaviors are illustrated and summarized in Figure 2-12. Corrugations on a sloping boundary involve four length scales, so can be fully described by three nondimensional parameters:

$$
\begin{aligned}
\frac{\delta}{A} & =\frac{\text { BL thickness }}{\text { Bump Height }} \\
\frac{\Delta h}{A} & =\frac{\text { Vertical Separation of Bumps }}{\text { Bump Height }} \\
\frac{A}{\lambda} & =\frac{\text { Bump Height }}{\text { Bump Length }}
\end{aligned}
$$



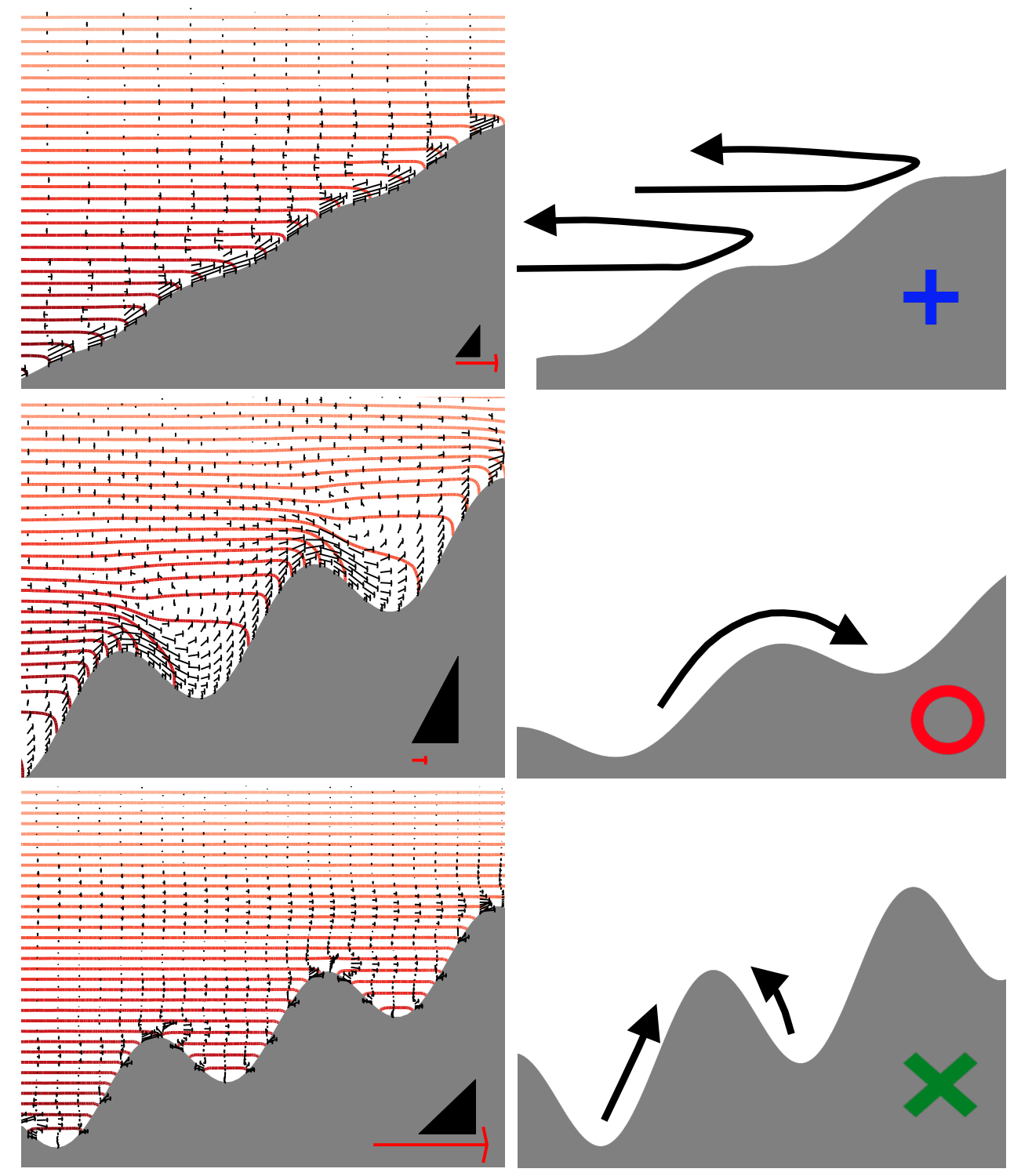

Figure 2-12: Three distinct types of flows have been observed in the numerical simulations, each shown with the symbol used in the summary plot in Figure 2-13. The smallest amplitude corrugations cause a series jets that exchange fluid between the boundary layer and the far field (top). As the topography becomes steeper, nonlinear effects become important, leading to overflows and spilling at each sill (middle). As the topography becomes taller still, a secondary up-slope boundary layer forms on the back face of each sill (bottom left). In these plots, the red arrow shows $2 \mathrm{~cm} / \mathrm{s}$ and the small black triangle is $1 \mathrm{~km}$ long and $50 \mathrm{~m}$ tall. 
As these parameter values change, we observe the three behaviors just described. The relationship between the nondimensional parameters and the flow behavior is summarized in Figure 2-13. As long as the vertical separation between adjacent sills is larger than the sill amplitude, the flow remains linear. As the amplitude of the topography becomes larger relative to that vertical separation and the mean boundary layer thickness, we see first overflows, then reversing boundary layers. Since there are different possible consequences for each type of boundary layer behavior, we would like to be able to predict when we expect to see each one.

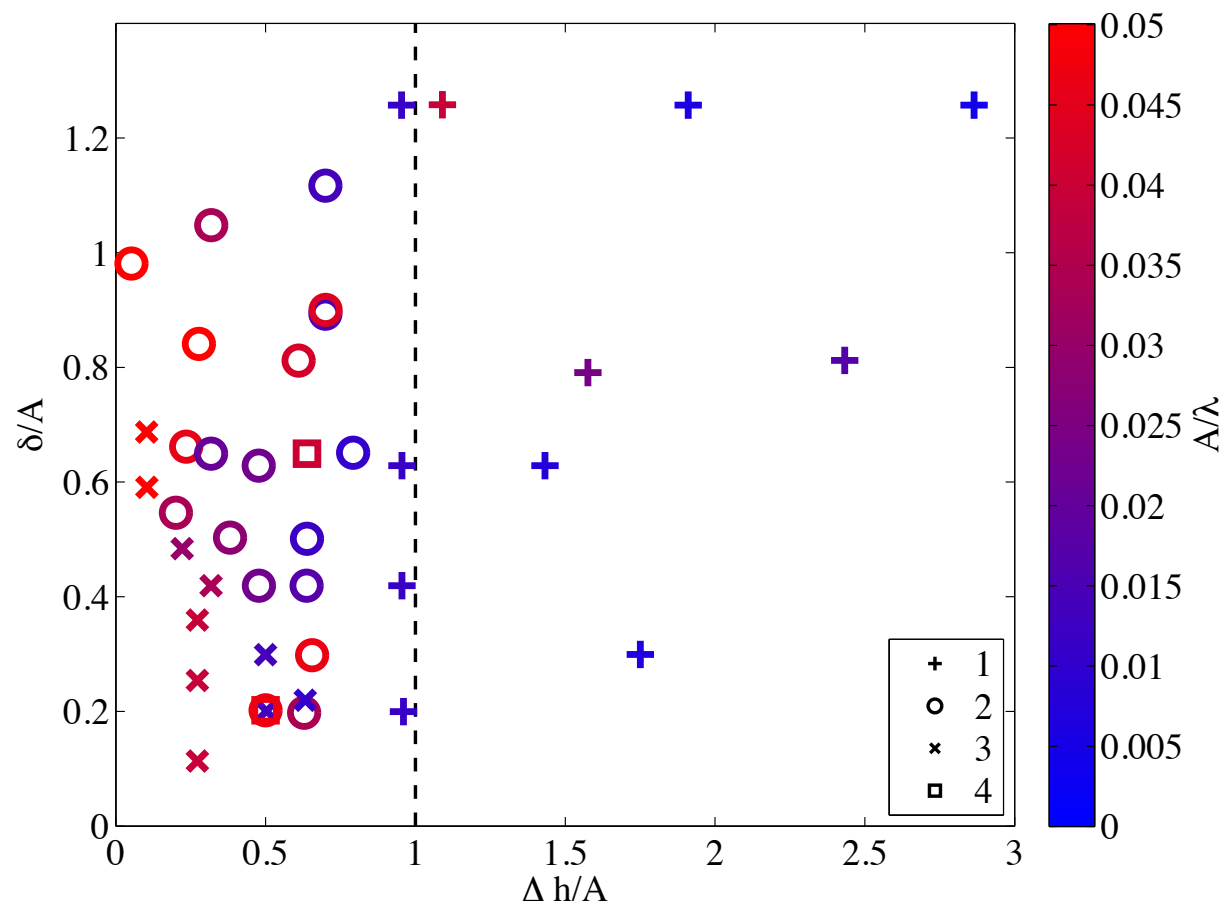

Figure 2-13: The behavior of the boundary layer over a corrugated, sloping bottom, as a function of the nondimensional topographic parameters: $\delta / A$, the boundary layer thickness relative to the topographic amplitude; $\Delta h / A$, the vertical separation between corrugations relative to the topographic amplitude; and $A / \lambda$, the aspect ratio of the corrugations. $\Delta h / A>1$ means that the bottom slope is always positive, and there is no trough behind the corrugation. In this parameter range, close to that of the ocean bottom, the behavior is most strongly predicted by $\delta / A$ and $\Delta h / A$. The symbols correspond to different flow behaviors, with typical examples of each given in Figure 2-12. 

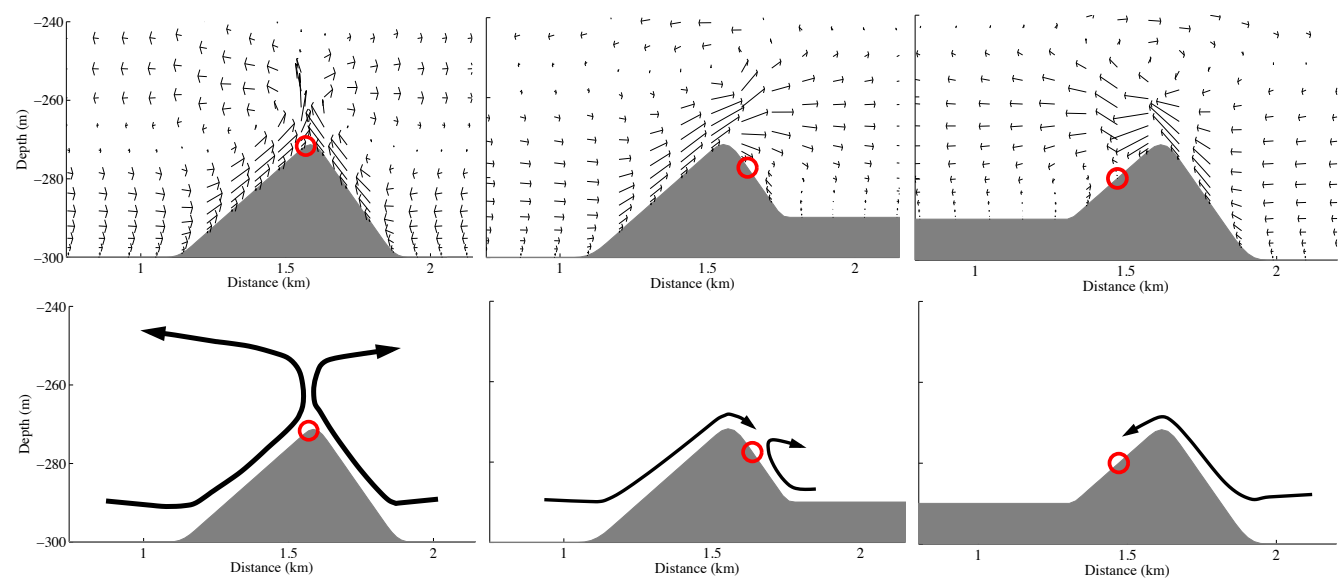

Figure 2-14: An asymmetrical bump with diffusive boundary layers causing upslope flows on both faces. The same shape bump can develop an overflow in either direction at the sill where none previously existed, depending on the length of each side of the sill. The red circle indicates the location of the bottom stagnation point in each case. I use that location to quantify the strength of the overflow.

\subsection{Predicting Bottom Boundary Layer Geometry}

By further simplifying our study topography, we can demonstrate that bottom stress is playing a key role in determining when overflows arise. In this section, I present two linked sets of numerical experiments that help us answer when overflows arise. This question is shown dramatically in figure 2-14, where we see three identical sills, each with left slope $\alpha=0.022$ and right slope $\alpha=0.062$. There is no background slope. Still, we are able to produce an overflow from the shallow side to the steep side, from the steep side to the shallow side, or no overflow at all, simply by changing the length of each side. Note that, as mentioned in Section 2.2 .2, since there is no mean slope, these numerical experiments used vertical walls for lateral boundaries.

The length of the slopes of the sill controls the overflow behavior because the boundary layer requires a finite horizontal scale to adjust to the Phillips solution: $L_{a d j}=2 \delta \cot \alpha$, given earlier in equation (2.2). We can see this asymptotic adjustment most clearly by looking at a boundary over a slope that emerges from a flat bottom, where no Phillips boundary layer exists (Figure 2-15). The boundary layer accelerates and adjusts to the 


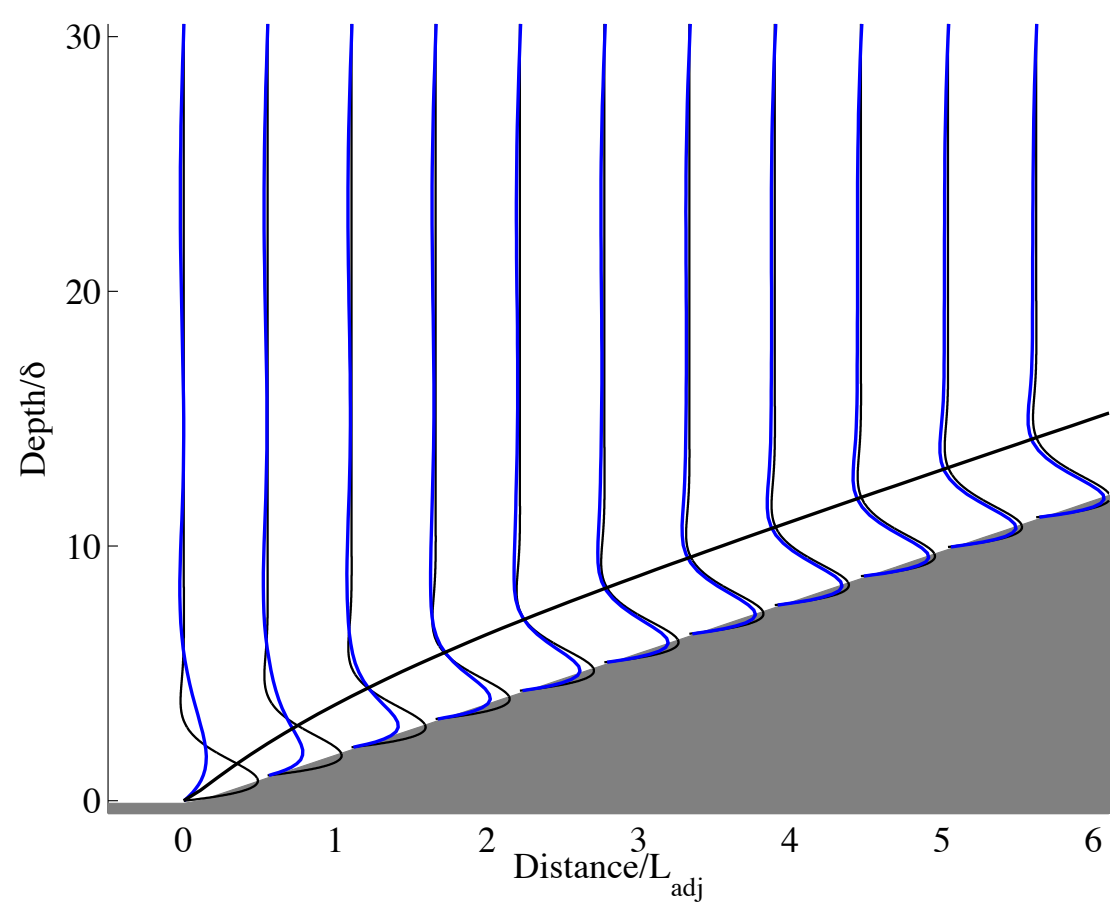

Figure 2-15: The boundary layer requires a well-defined length to accelerate to its constant-slope solution. Thin black lines are the theoretical (Phillips) solution velocity, and the colored lines are the computed velocity profiles. The thick black line shows the predicted thickening of the BL as it accelerates.

Phillips solution over a well-defined distance $L_{a d j}$ as you move up the slope.

We can use the adjustment behavior of a boundary layer over a single constant slope (like in Figure 2-15) to understand the behavior at a sill (like in Figure 2-14). The idea is shown in schematic form in Figure 2-16: we can take a profile from our single slope case to understand how the boundary layer on one side of the bump would behave if the other side of the bump were not there.

We find that almost everywhere in the water column, the velocities associated with the shallower slope are faster than those associated with the steeper slope (Figure 2-17). This is true even when there is an overflow from the steep side to the shallow side.

However, if we look at vertical shear of horizontal velocity instead of horizontal velocity, the different flow behaviors separate. We can calculate an estimated bottom stress by comparing the velocities from the constant-slope simulations that correspond to the slopes 


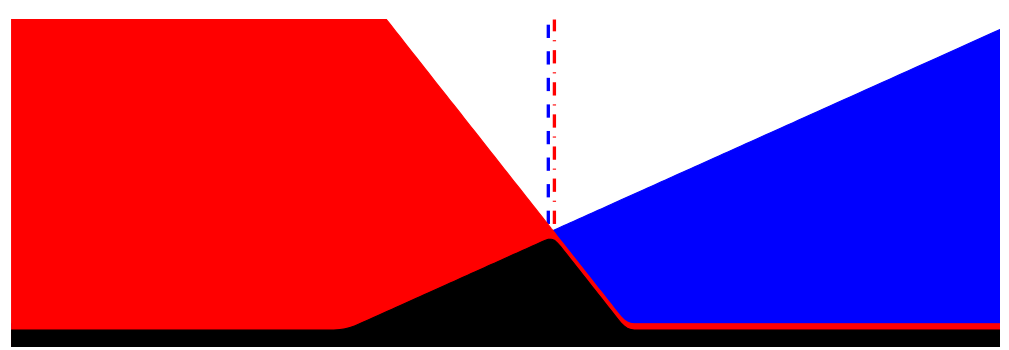

Figure 2-16: We can use boundary layer simulations over single constant slopes (blue and red topography) to understand the behavior of boundary layers over asymmetrical bumps (black topography). A profile taken over each of the constant slopes along the dashed line would give us an idea of the behavior of the boundary layer at the sill if the other side of the bump were not there. Comparisons of the profiles associated with each side for the simulations in Figure 2-14 are shown in Figure 2-17.

on each side of an asymmetrical bump:

$$
\text { Estimated Bottom Stress }=\left.\nu \frac{\partial}{\partial z}\left(u_{\text {shallow }}\left(y_{\text {sill }}\right)-u_{\text {steep }}\left(y_{\text {sill }}\right)\right)\right|_{z=\text { bottom }}
$$

That is, we take the difference between the corresponding red and blue profiles in Figure 2-17, and use that to calculate a bottom stress at the sill. Strong overflows are associated with estimated bottom stresses in the direction of the overflow, while runs without an overflow have little or no estimated bottom stress at the sill. This makes sense because at the sill the primary momentum balance is between pressure and stress. The estimated bottom stress at the sill therefore tells us the direction of the pressure gradient at the sill.

We can quantify the relationship between the bottom stress and the strength and direction of an overflow by comparing the virtual bottom stress to the location of the stagnation point on the bottom boundary (Figure 2-18). If there is no overflow, the stagnation point should be exactly at the location of the sill. As an overflow forms, the stagnation point is pushed to one side of the sill or the other. How far the stagnation point is pushed tells you how strong the overflow is. The stagnation point location is taken from the numerical experiments of asymmetrical bumps, illustrated in the schematic diagrams in Figure 2-14. The bottom stress (proportional to the bottom shear) is calculated from the numerical experiments of boundary layers developing over constant slopes, like the one shown in 


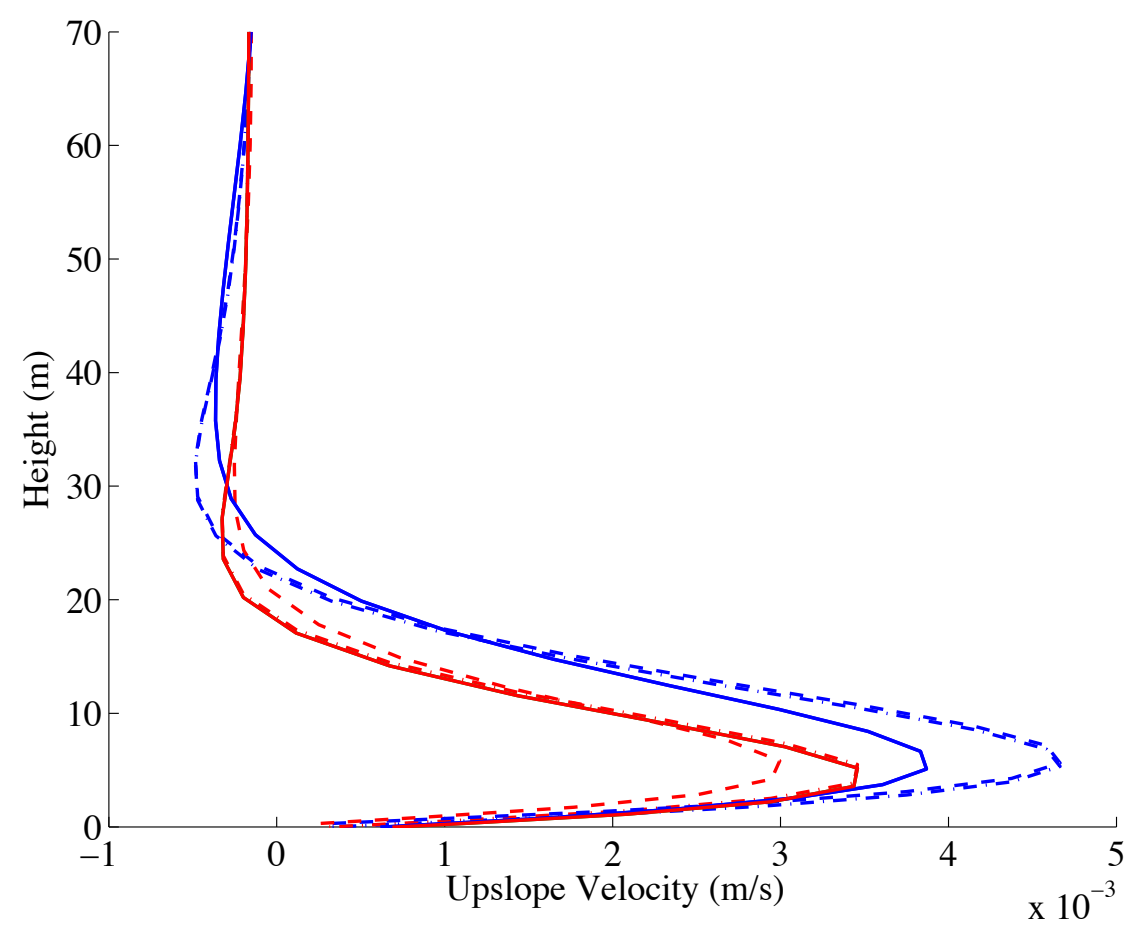

Figure 2-17: The boundary layer velocities associated with each side of a bump show that the shallower slope (blue) has a faster current throughout the boundary layer. These profiles are calculated as diagrammed in Figure 2-16, using the three simulation in Figure $2-14$. Even though the blue velocity is nearly always greater, we can generate an overflow from steep to shallow (red to blue).

Figure 2-15. That is, they are calculated independently of one another. However, we find that this estimated bottom stress is able to explain more than $70 \%$ of the variance of the location of the stagnation point and so of the strength and direction of the overflow. This suggests very strongly that bottom stress may be a determinative factor in whether or not an overflow forms.

This relationship is consistent with previous theories of laminar boundary layer separation. Prandtl [1905] was the first to observe that laminar boundary layers separate where the bottom stress goes to zero. The boundary layer should flow continuously until it reaches that point. Therefore, it the zero-bottom-stress point is downstream of the sill, we expect an overflow. The location of the stagnation point and so the strength and direction of the 


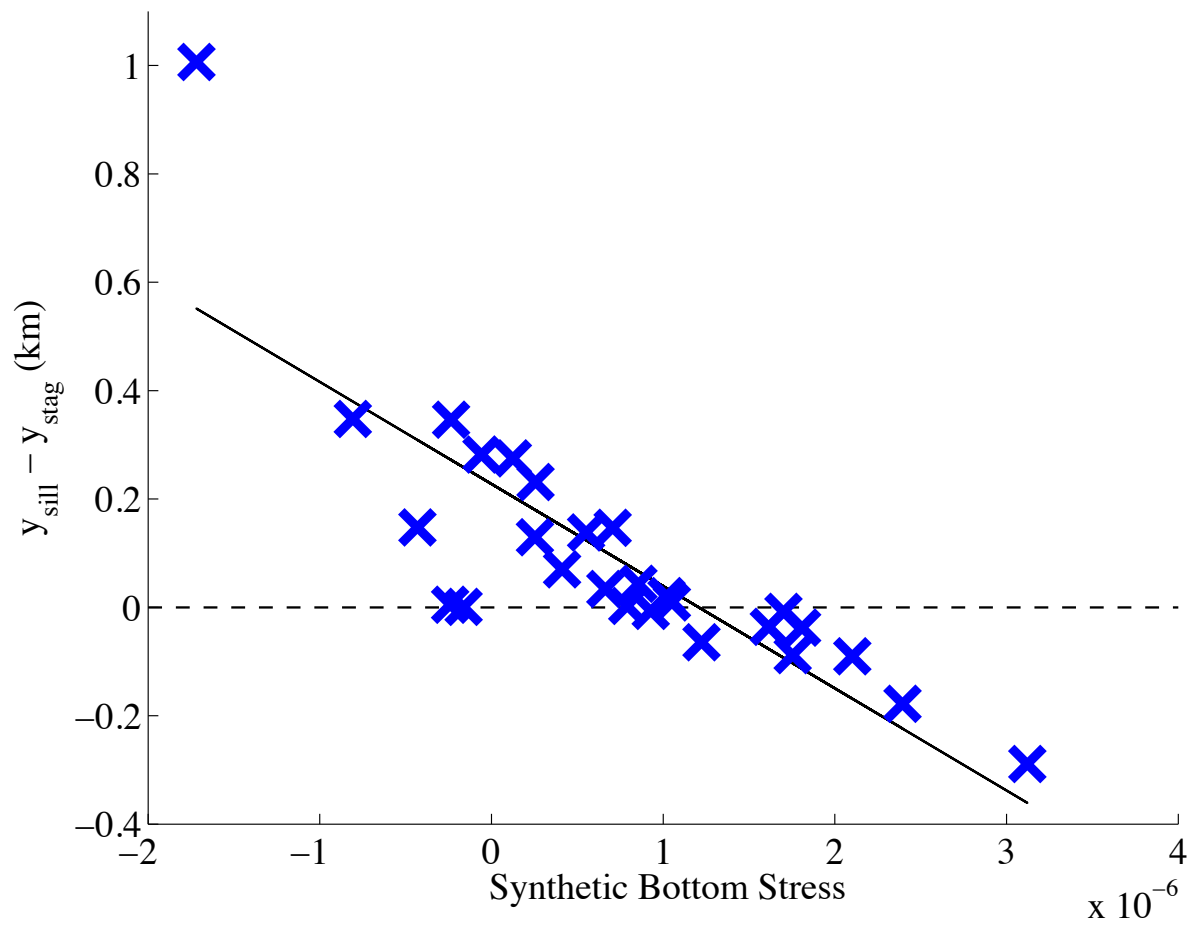

Figure 2-18: The estimated bottom stress, composed from simulations on constant slopes, is strongly correlated with the strength of an overflow, as indicated by the location of the stagnation point in the boundary layer over an asymmetrical bump relative to the location of the top of the bump (the sill). The values on each axis come from different sets of numerical experiments and are calculated independently. This suggests that bottom stress may be a big part of the overflow story. Note that $70 \%$ of the variance is explained by a simple linear fit. The different colors are associated with different groups of numerical experiments with different bottom slopes.

overflow is:

$$
y_{\text {stag }}=m\left(\left.\frac{\partial}{\partial \eta}\left(u_{1}-u_{2}\right)\right|_{\eta=0}\right)+b
$$

where $m=942$ and $b=0.228$, which define the least-squares fit line (Figure 2-18).

There are two available ways to estimate the bottom stress at the sill. First, we can calculate it numerically as we did in Figure 2-18, using simulations like that in Figure 215. These show that the bottom shear asymptotes to its final value over a length scale of approximately $L_{a d j}$ (Figure 2-19). Alternatively, we can take advantage of this information to calculate it from quantities we know a priori. The bottom stress for the analytical Phillips 


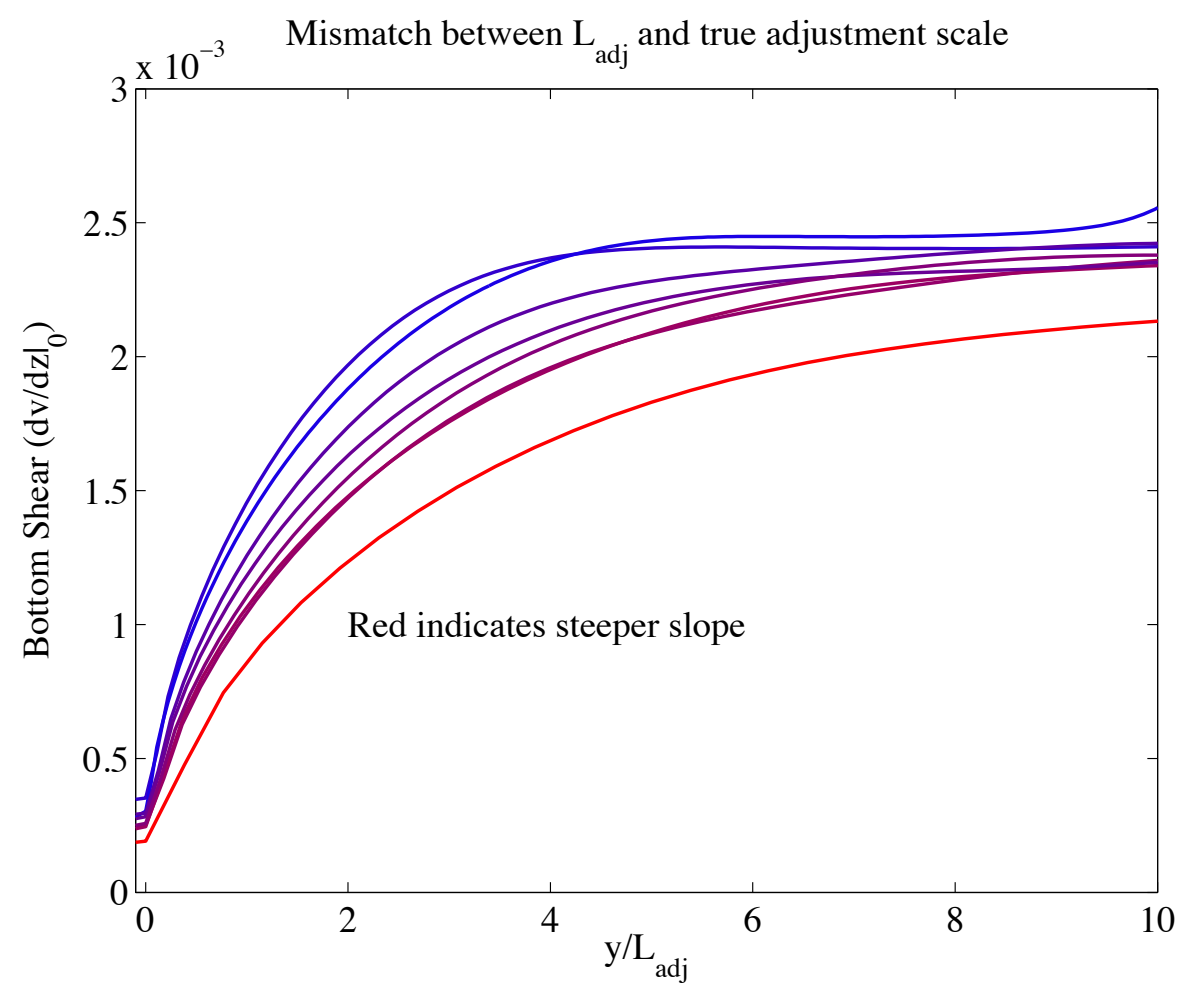

Figure 2-19: The bottom shear as a function of along-slope distance is similar to the prediction (equation 2.6), but disagrees in a couple noteworthy ways. First, the adjustments do not collapse completely based on the scaling $L_{a d j}$; they disagree systematically based on slope. Here redder is steeper and bluer is shallower, and the red is separated from the blue. Second, their final value is not that given from the Phillips solution in equation (2.5). For these parameters, the final value should be $3.16 \times 10^{-3}$, and the numerics consistently produce a value $20 \%$ less. Note that this has negligible effect on the final result for the corrugations.

solution (from equation 1.11) is:

$$
\tau_{0}=\left.\nu \frac{\partial u}{\partial \eta}\right|_{\eta=0}=2 \nu \frac{\kappa}{\delta^{2}} \cot \alpha=\frac{\nu N \cos \alpha}{\sqrt{\mathbb{P}_{r}}}
$$

Conveniently, $\tau_{0}$ is constant in the small angle limit, which is what we care about on the bottom of the ocean. As a boundary layer develops, we assume that the bottom stress will act like:

$$
\left.\nu \frac{\partial u}{\partial \eta}\right|_{\eta=0}=\nu \tau_{0}\left(1-e^{-\xi / L_{a d j}}\right)
$$




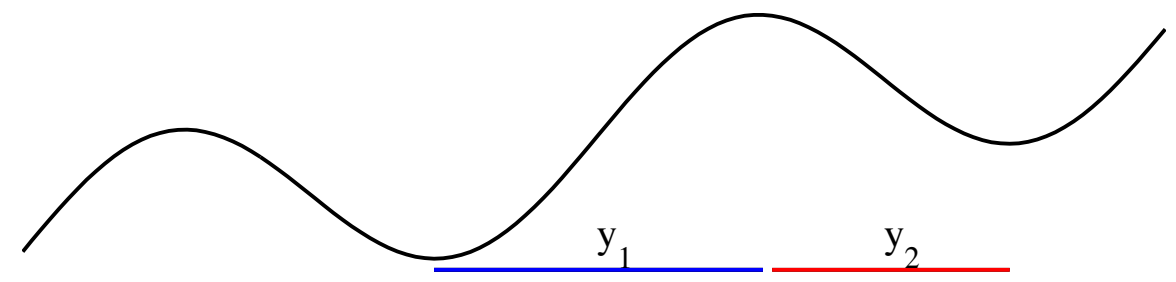

Figure 2-20: Each corrugation can be approximated as an asymmetrical bump. The lengths of the sides are approximated as $y_{1}$ and $y_{2}$, and the slopes are taken from the steepest part of each face of the corrugation.

where here $\theta=L_{a d j}$. We know all of the quantities in this expression without numerical computation. The numerically computed values of both the bottom stress $\tau_{0}$ and the adjustment scale $\theta$ are within $20 \%$ of the predicted values for a range of slopes spanning more than an order of magnitude $(\alpha=0.01-0.2)$. More complicated expressions for $\theta$ improve the agreement in Figure 2-19 somewhat, but this introduces extra complexity with poor physical justification and does not significantly improve the final prediction of which corrugations cause overflows.

To make this prediction, I apply equation (2.6) to the corrugated slopes by treating each sill as one of the asymmetrical bumps, with the length of each side give by $y_{1}$ and $y_{2}$ in Figure 2-20 and $\alpha_{1}$ and $\alpha_{2}$ given by the steepest part of the respective slopes. We substitute the value for the bottom stress in equation (2.6) into our expression for the location of the stagnation point (equation 2.4), giving:

$$
y_{\text {stag }}=m \sigma_{0}\left(e^{-y_{2} / \theta_{2}}-e^{-y_{1} / \theta_{1}}\right)+b
$$

If $y_{\text {stag }}>0$, we expect an overflow. We can map this onto our regime diagram to predict what corrugations will produce overflows (Figure 2-21). The $y_{\text {stag }}=0$ contour separates the reversing boundary layers from the overflows quite well. Note that we do not expect the contour to separate them perfectly, as the prediction is based on two constant slopes meeting at a point, and our corrugations vary continuously in slope. 


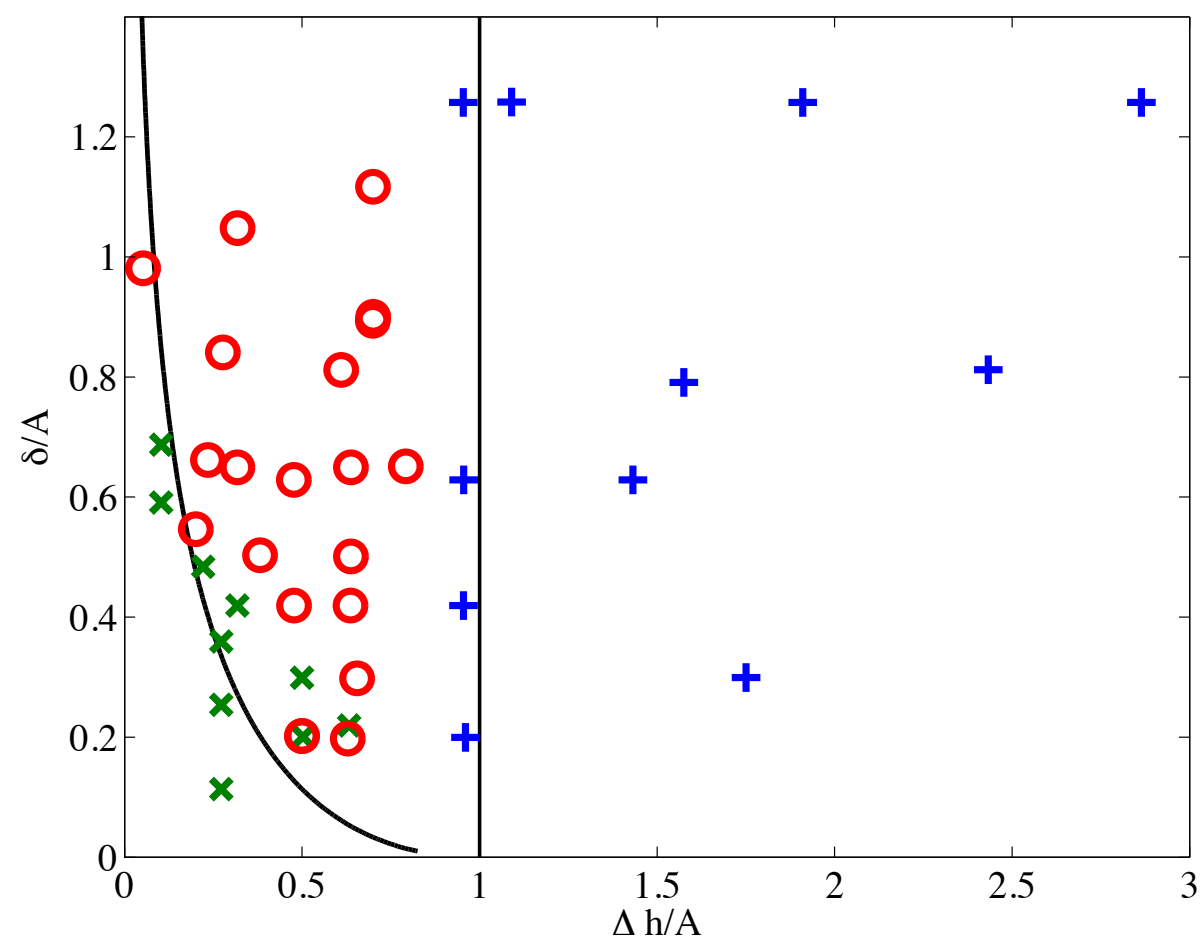

Figure 2-21: The flow geometry prediction compares favorably to the results of the numerical experiments. The vertical black line separates the expected linear runs from the others. The curved black line is the prediction of the boundary between the overflows and the reversing boundary layers. The prediction is not perfect, but it cannot be expected to be since the actual topography is more complicated than two constant slopes. However, it is approximately correct and it is derived from first principles. All the hoo-hah with the asymmetrical bumps and the numerics for constant-slope adjustment lead us to this result, but was ultimately not used to draw this trace.

\subsection{Conclusions}

Even simple changes in topography can lead to a wide variety of different dynamics in diffusive bottom boundary layers. Any change in slope forces an exchange of fluid between the boundary layer region and the far-field, and certain types of topography can lead to acceleration and advection in the boundary layer itself.

These types of boundary layers may play an important role in abyssal mixing and the heat and mass budgets of the deep ocean basins. Many scientists have observed extremely high levels of turbulence and mixing at topographic overflows [Ferron et al., 1998, Bry- 
den and Nurser, 2003]. As mentioned in the introduction, in the Romanche Fracture Zone, Polzin et al. [1996] observed turbulent dissipation at a sill that was 10,000 times stronger than the thermocline background. Other authors have speculated about whether smaller canyons, like that of the BBTRE, might feature overflows like those observed in larger fracture zones [Thurnherr et al., 2005]. We know that there are sills and obstructions in many of these canyons, but we do not have conclusive observations of topographic interactions. No one has ever really looked.

This work demonstrates that overflows at sills do not require far-field forcing such as exists in the passage of the Antarctic Bottom Water through the Romanche Fracture Zone or the Denmark Strait. Given appropriate topography, overflows can develop powered only by the heat flux from a weak background stratification. Typical topography in mid-ocean ridge canyons, based on the high-resolution bathymetry measured in the $22^{\circ} \mathrm{S}$ canyon, falls in the same area of nondimensional parameter space where these overflows are predicted (Figure 2-21). Therefore, these numerical studies suggest that overflows forced by stratification are robust, and may be ubiquitous in the deep ocean.

\section{A Appendix - Effects of $\kappa, \nu$ variation on a Constant Slope Boundary Layer}

I developed a one-dimensional numerical solver to calculate the structure of the boundary layer when the mixing coefficients vary with distance from the boundary, that is:

$$
\kappa=\kappa(\eta) \quad ; \quad \nu=\nu(\eta)
$$

To validate the solver, I found an analytical solution in the case where $\nu, \kappa$ are piecewise constant:

$$
\kappa, \nu=\left\{\begin{array}{lll}
\kappa_{1}, \nu_{1} & \text { for } & \eta<\eta_{0} \\
\kappa_{2}, \nu_{2} & \text { for } & \eta>\eta_{0}
\end{array}\right.
$$


Since the viscosity and diffusivity are everywhere locally constant, the governing equation can be taken directly from the constant coefficient case in equation (1.9):

$$
r^{\prime \prime \prime \prime}+4 \gamma_{P}^{4} r=0
$$

Recall $r(\eta)$ is the density perturbation, defined in equation (1.8). The solutions are of the form of growing and decaying sines and cosines. Without loss of generality, and with great increase in convenience, we can express the growth and decay as sines and cosines decaying away from the bottom and decaying away from the interface $\eta_{0}$ :

$$
\phi(\eta)=A e^{-\gamma \eta} \sin \gamma \eta+B e^{-\gamma \eta} \cos \gamma \eta+C e^{-\gamma\left(\eta_{0}-\eta\right)} \sin \gamma \eta+D e^{-\gamma\left(\eta_{0}-\eta\right)} \cos \gamma \eta
$$

The coefficients $A-D$ are different for the two regions. The resulting eight coefficients are determined with four bottom boundary conditions and four matching conditions at the interface between the two regions at $\eta=\eta_{0}$. The boundary conditions are the same as those used by Phillips in equations (1.5) - (1.7). Physically, they are no buoyancy flux through the bottom boundary, no slip at the bottom boundary, and boundedness of the solution everywhere in the domain (which accounts for two boundary conditions).

The matching conditions at the interface between the two regions are a little more subtle. They are found by integrating the governing equation for freely varying viscosity and diffusivity:

$$
r g N^{2} \sin ^{2} \alpha+\left(\nu\left(\kappa g r^{\prime}-\kappa N^{2} \cos \alpha\right)^{\prime \prime}\right)^{\prime}=0
$$

We integrate this equation across an infinitesimal interval that spans the boundary between the to regions, $\left(\eta_{0}-\epsilon, \eta_{0}+\epsilon\right)$. Since this interval is infinitesimal, any finite quantity integrated across it is zero. The first integration gives:

$$
\left.\nu\left(\kappa g r^{\prime}-\kappa N^{2} \cos \alpha\right)^{\prime \prime}\right|_{\eta_{0}-\epsilon} ^{\eta_{0}+\epsilon}=0
$$


From this, we find our first matching condition:

$$
\nu_{1} \kappa_{1} r_{1}^{\prime \prime \prime}\left(\eta_{0}\right)=\nu_{2} \kappa_{2} r_{2}^{\prime \prime \prime}\left(\eta_{0}\right)
$$

This can be physically interpreted as the smoothness of the velocity field or conservation of stress across the interface, as the velocity is proportional to $\kappa r^{\prime \prime}(\eta)$. The next matching condition comes from a second integration of equation (2.11) over the same interval:

$$
\kappa_{1} r_{1}^{\prime \prime}\left(\eta_{0}\right)=\kappa_{2} r_{2}^{\prime \prime}\left(\eta_{0}\right)
$$

This is a statement of the continuity of velocity across the interface. A third integration gives:

$$
\kappa_{1} r_{1}^{\prime}\left(\eta_{0}\right)-\frac{\kappa_{1} N^{2} \cos \alpha}{g}=\kappa_{2} r_{2}^{\prime}\left(\eta_{0}\right)-\frac{\kappa_{2} N^{2} \cos \alpha}{g}
$$

This is the conservation of buoyancy flux across the interface. A fourth and final integration across the interface gives:

$$
r_{1}\left(\eta_{0}\right)=r_{2}\left(\eta_{0}\right)
$$

The density field is continuous across the interface. Given these boundary and matching conditions, it is possible to solve for the full density profile. The velocity profile can be inferred from the density profile.

Physically, we expect to observe the up-slope bottom boundary layer predicted in the constant $\kappa, \nu$ case and an additional internal boundary layer at the interface between the two regions. The internal boundary layer arises because the buoyancy fluxes in the two regions are different — after all, they have different diffusivities and the same stratification—leading to a buoyancy flux divergence at the interface. In order to conserve mass, there must be an advective mass flux at the interface, bringing dense fluid up if $\kappa_{1}<\kappa_{2}$ or light fluid down if $\kappa_{1}>\kappa_{2}$. Another way to think about this is in terms of the far-field constraint on the boundary layer flux, equation (2.1). If the interface is far from the bottom boundary, we expect the bottom boundary layer to behave like it would if the diffusivity is a constant 
$\kappa=\kappa_{1}$, with a volume flux of $Q=\kappa_{1} \cot \alpha$. However, the overall volume flux in the whole boundary layer system must be determined by $\kappa_{\infty}=\kappa_{2}$. This means that there must be a volume flux associated with the change in diffusivity given by:

$$
Q_{I B L}=\left(\kappa_{2}-\kappa_{1}\right) \cot \alpha
$$

$Q_{I B L}$ is the volume flux in the internal boundary layer at $\eta=\eta_{0}$. In the real ocean, we expect mixing to be bottom-intensified $\left(\kappa_{1}>\kappa_{2}\right)$, so the flux in the internal boundary layer is flowing down-slope.

The structure of the solution can be further illuminated by nondimensionalizing it. The relevant non-dimensional parameters express the change in the diffusivity and viscosity at the interface and the location of of the interface:

$$
\begin{aligned}
& X_{1}=\left(\frac{\kappa_{1}}{\kappa_{2}}\right)^{1 / 4} \\
& X_{2}=\left(\frac{\nu_{1}}{\nu_{2}}\right)^{1 / 4} \\
& X_{3}=\gamma_{1} \eta_{0}
\end{aligned}
$$

The coefficients $A-D$ in equation (2.10) all depend on these three non-dimensional parameters. As the interface moves away from the boundary $\left(X_{3} \gg 1\right)$, the internal boundary layer velocity is given by:

$$
v(\zeta)= \begin{cases}2 e^{\zeta \frac{\left(X_{1}^{4}-1\right)\left(-X_{2}^{2}\left(1+X_{1} X_{2}\right) \cos \zeta+X_{1}\left(X_{1}+X_{2}^{3}\right) \sin \zeta\right)}{X_{1}^{2}\left(X_{1}^{4}+2 X_{1} X_{2}+2 X_{1}^{2} X_{2}^{2}+2 X_{1}^{3} X_{2}^{3}+X 2^{4}\right)}} & \text { for } \quad \zeta<0 \\ -2 e^{-X_{1} X_{2} \zeta \frac{\left(X_{1}^{4}-1\right) X_{2}^{2}\left(X_{1}\left(1+X_{1} X_{2}\right) \cos \left(X_{1} X_{2} \zeta\right)+\left(X_{1}+X_{2}^{3}\right) \sin \left(X_{1} X_{2} \zeta\right)\right)}{X_{1}^{3}\left(X_{1}^{4}+2 X_{1} X_{2}+2 X_{1}^{2} X_{2}^{2}+2 X_{1}^{3} X_{2}^{3}+X 2^{4}\right)}} & \text { for } \quad \zeta>0\end{cases}
$$

$\zeta=\gamma_{1}\left(\eta-\eta_{0}\right)$. You can see immediately that if $X_{1}=1$, that is if $\kappa_{1}=\kappa_{2}$, the velocity in the internal boundary layer is zero, and the internal boundary layer ceases to exist. If there is no divergence of mass flux at the interface, there is no need for advection to conserve mass, so there is no impetus for the internal boundary layer to form in the first place. 

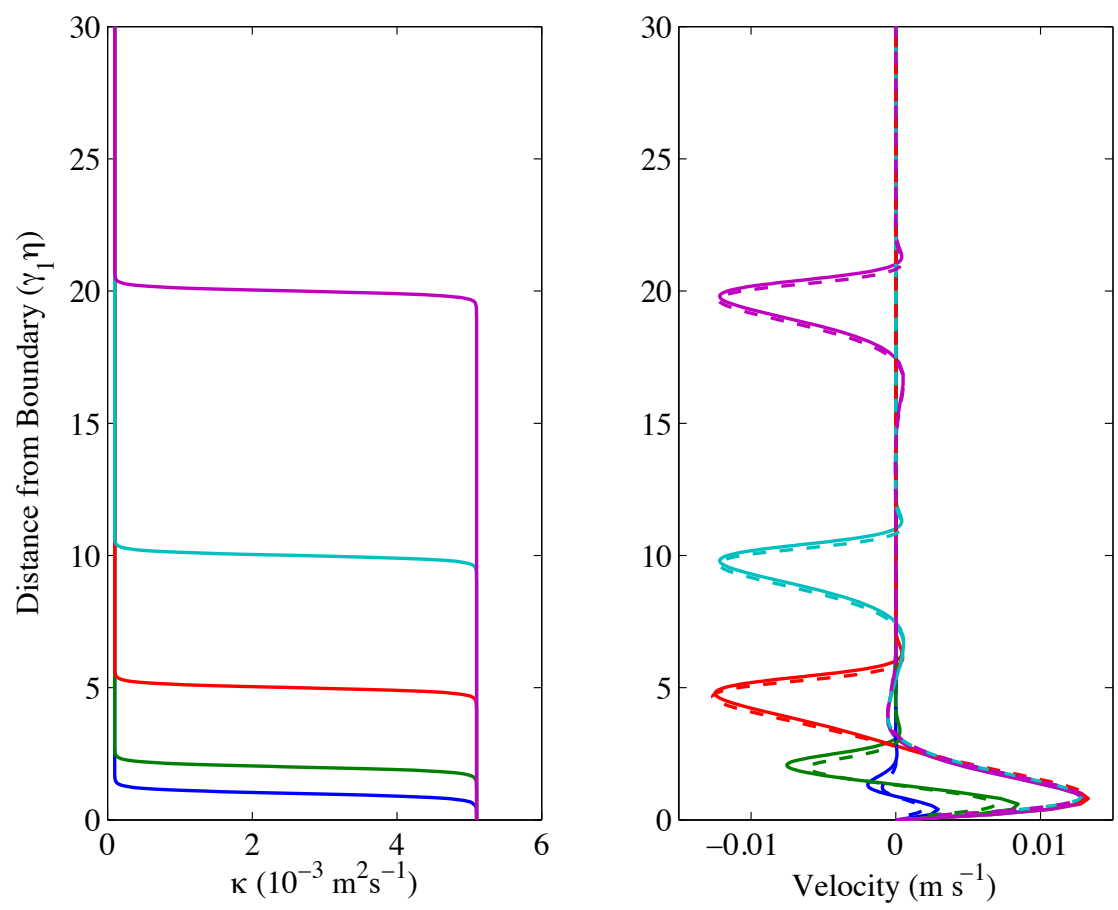

Figure 2-22: Over a constant slope, a piecewise constant diffusivity $\kappa$ leads to the formation of an interior boundary layer at the height of the jump in $\kappa$. This is necessary to satisfy the far-field constraint on the boundary layer flux and to prevent divergences in mass flux. In this way, we get large velocities induced by the mixing field far from the boundary. This plot shows steps in $\kappa$ at a variety of heights and the corresponding up-slope velocity profile. Height is measured in boundary layer thicknesses. We also compare the analytical solution for piecewise constant $\kappa$ (dashed line) to the numerical solution (solid line), and see that our numerical solver is reliable.

The full analytical solution for piecewise-constant mixing coefficients (both more general and more complicated than equation 2.21) compares very favorably to the solution from the one-dimensional numerical solver (Figure 2-22). Since it succeeds in this numerically stiff case, we can use the numerical solver confidently for a wide variety of $\kappa=\kappa(\eta), \nu=\nu(\eta)$. The solver works when $\nu, \kappa$ vary smoothly everywhere and approach a constant asymptotic value very far from the boundary. The numerical solver incorporates a boundary value solver in Matlab to solve equation (2.11).

What we find in both the piecewise constant and smoothly varying cases is that changes in $\nu, \kappa$ can cause substantial velocities much farther from the boundary than predicted by 

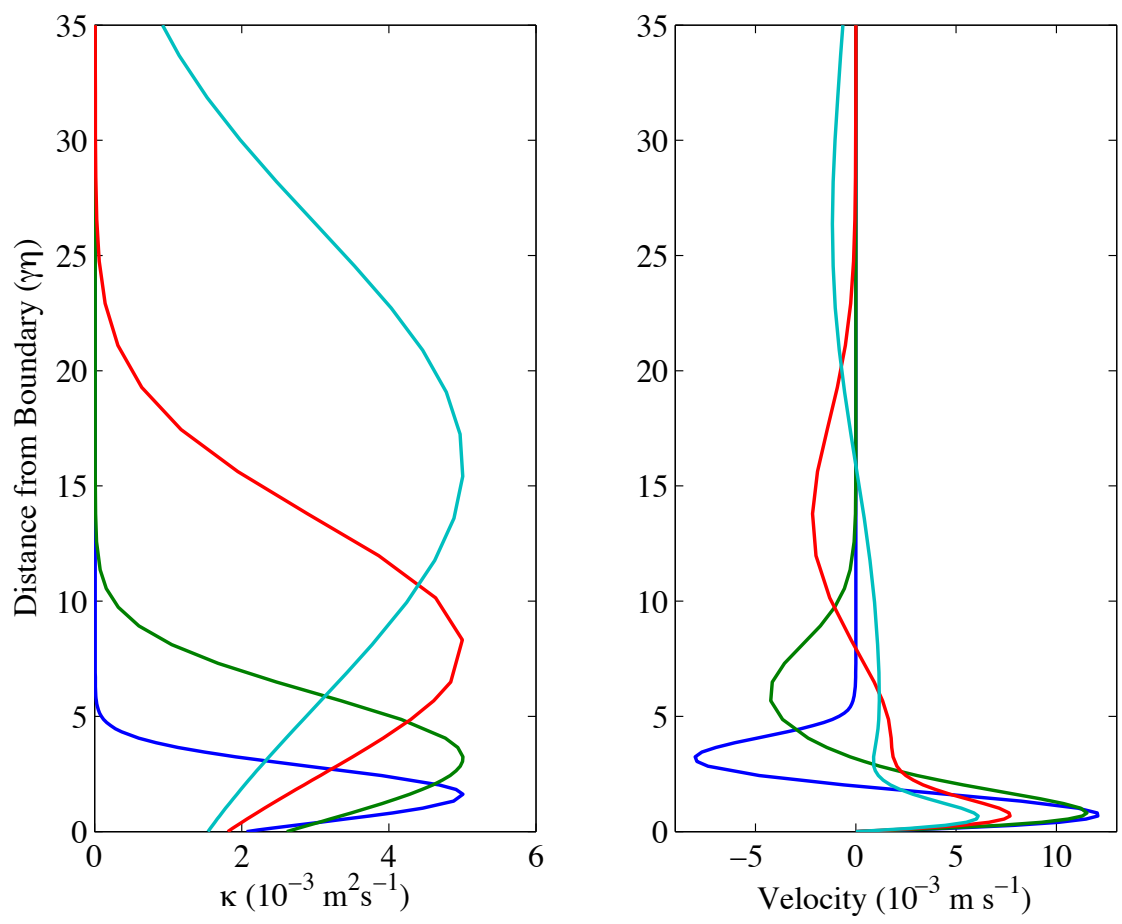

Figure 2-23: Boundary layer behavior when mixing coefficients $\nu, \kappa$ vary smoothly with distance from bottom. When there is a maximum in the mixing strength above the bottom boundary, the effects of the boundary layer can be felt much higher in the water column. The left plot shows some profiles of $\kappa$, while the plot on the right shows the resulting up-slope velocity.

Philips's boundary layer scale thickness from equation (1.12):

$$
\delta=\left(\frac{4 \nu \kappa}{N^{2} \sin ^{2} \alpha}\right)^{\frac{1}{4}}
$$

In the piecewise constant case, the fluid motion far from the bottom boundary is in the form of the internal boundary layer at $\eta_{0}$ (Figure 2-22). In the smoothly varying case, the boundary layer velocity also varies smoothly on the scale of variations in $\nu, \kappa$ (Figure 2-23). This effect might be relevant to explaining the thickness of the boundary layer observed in oceanic canyons. In addition to providing interesting insight into the boundary layer, this solution may be useful in the future for calculating appropriate boundary conditions for future ROMS simulations with varying $\kappa, \nu$. 


\section{B Appendix - Jet model}

The 'blob' or area of low stratification that we referred to earlier in Figure 2-11 may explain the large thickness of the boundary layer observed in abyssal density fields. We can think of a system of two nested boundary layers: the inner layer is the classic Phillips diffusive boundary layer, and the outer layer is the transitional area effected by the fluid exchange between the inner layer and the far-field fluid. In Figure 2-3, you can see the inner layer is less than $50 \mathrm{~m}$ thick, while there's a transitional region of perhaps 500m thickness. The outer layer is more similar to the observed thickness of the abyssal bottom boundary layer.

To better understand the exchange between the boundary layer and the far-field, consider a further simplified problem: a horizontal neutrally buoyant jet coming from a pointsource. An intrusion coming out of the boundary layer is something like a horizontal jet, and in the linear limit, an entrainment flow is as well. A jet is governed by the same diffusive Boussinesq dynamics as the Phillips boundary layer. This jet is a straightforward simplification of the broken slope scenario. The linear corrugated slope may be thought of as a stack of jets from sources and sinks along the boundary.

In an infinite domain, this jet can be described in terms of a similarity variable $\zeta=\frac{z}{x^{1 / 3}}$. In terms of this variable, the non-dimensional streamfunction $f(\zeta)=\psi / \Psi_{0}$ satisfies:

$$
f^{(6)}+\frac{4}{9} \zeta f^{(1)}-\frac{1}{9} \zeta^{2} f^{(2)}=0
$$

This jet is straightforward to model numerically (Figure 2-24).

It's shape follows the similarity variable. Dimensionally:

$$
z^{3} \sim x \frac{\sqrt{\nu \kappa}}{N}
$$

This scaling holds true for numerical models of jets with a variety of values of $N, \nu, \kappa$ and also a variety of aperture sizes for the fluid source (Figure 2-25). From this scaling, we can estimate the thickness $D$ of the transitional region or outer boundary layer. $D$ is the 


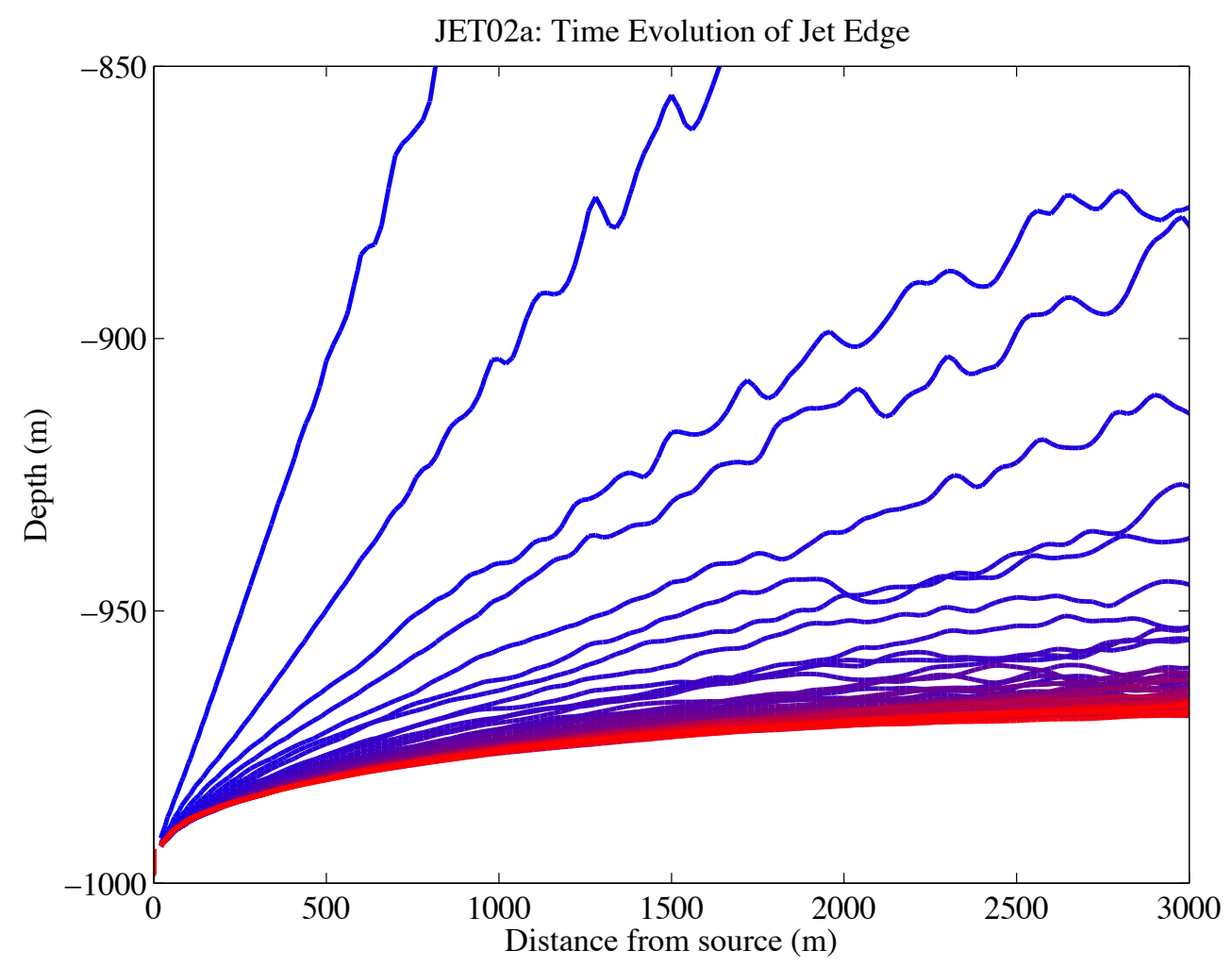

Figure 2-24: A horizontal neutrally-buoyant jet focuses with time. The lines show the outer edge of the jet, moving from blue to red as time passes. The jet reaches a steady state in shape, clearly showing the scaling of the similarity variable $\zeta=\frac{z}{x^{1 / 3}}$.

distance over which the vertical jet thickness becomes the same as the vertical separation between a source jet and an adjacent sink jet. That is, it is the distance over which jets that are adjacent in the vertical come to overlap and cancel each other out. That thickness scales like:

$$
D \sim \alpha^{4} \lambda^{3}\left(\frac{N^{2}}{\nu \kappa}\right)^{1 / 2}
$$

where $\alpha$ is the mean slope. Since $D$ depends so sensitively on it, a very wide range of thicknesses are consistent with reasonable values of $\alpha$. This range includes the $500 \mathrm{~m}$ that we observe in the ocean. 


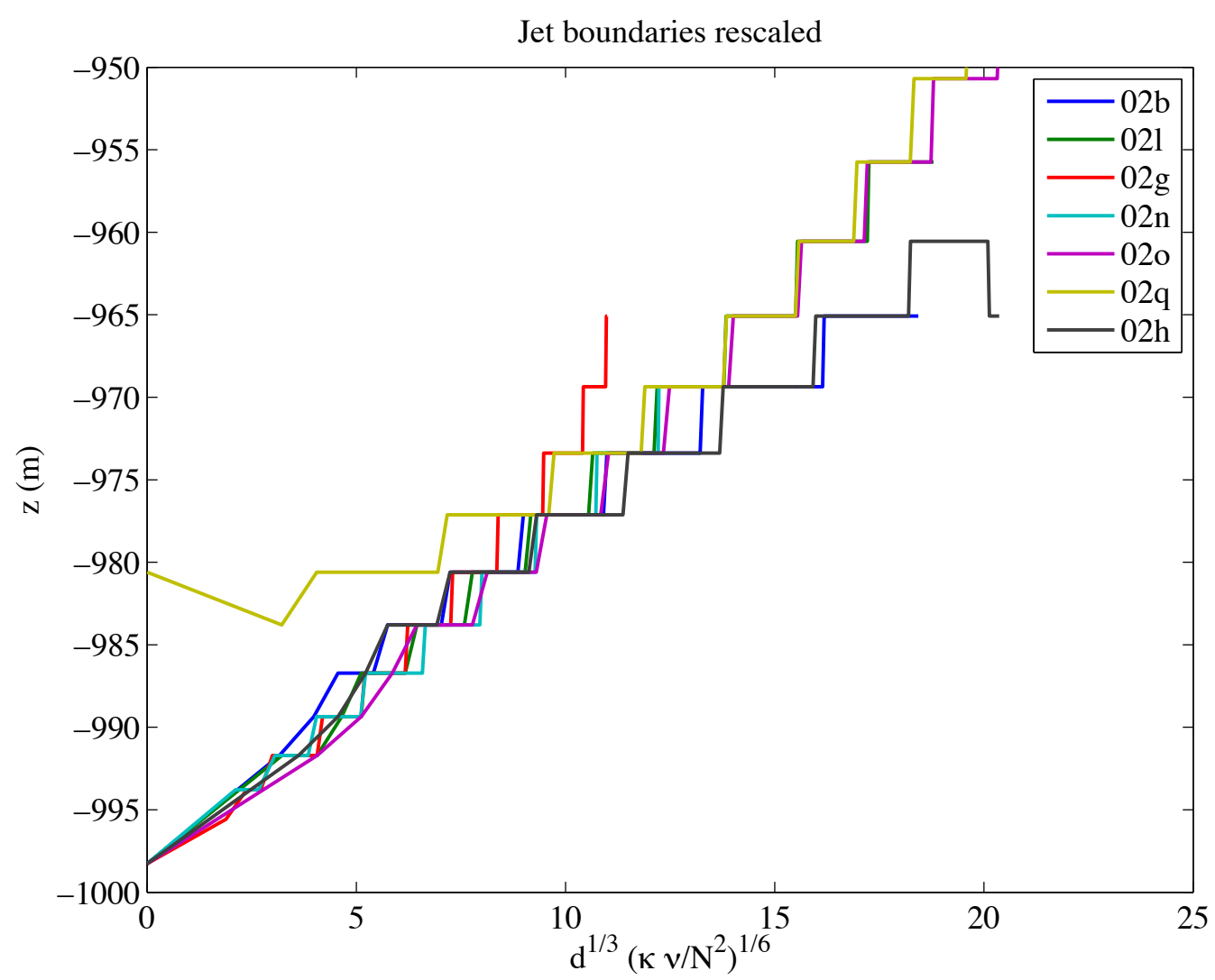

Figure 2-25: A number of modeled jets, with varying $N, \nu, \kappa$, nozzle size, and nozzle velocity all show the predicted scaling of the jet shape. Each line shows a model run, and when properly rescaled the collapse onto a single straight line. This scaling can then be used to estimate the thickness of the transitional region or outer boundary layer between the Phillips layer and the far field.

\section{C Appendix - Studying the Effects of Topography in the Laboratory}

The variety of boundary layer geometries found in the numerical simulations of diffusive boundary layers presented in this chapter have not been described previously. As a new class of fluid phenomena, we would like to produce them in the laboratory. In this appendix, I will present the key challenges of these experiments and show how they might be addressed.

The first challenge is resolving velocity field in the boundary layer. In the laminar case, 
using molecular diffusivity and viscosity, the boundary layer is only about $1 \mathrm{~mm}$ thick. Its thickness is limited both by the small values of $\nu$ and $\kappa$, and by the small slope limit for effectively establishing the boundary layer. Peacock et al. [2004] report that below about $4^{\circ}$, the boundary layer no longer conforms to the predictions of Phillips [1970]. Allshouse et al. [2010] produce the predicted boundary layers at slopes of about $5^{\circ}$. To ensure that we do produce the desired boundary layer, I recommend taking a slope not significantly below:

$$
\alpha=5^{\circ}
$$

Allshouse et al. [2010] also discusses how non-traditional salts can be used to increase the diffusivity and glycerol mixtures can increase the viscosity. With these techniques, we should be able to use the following mixing coefficients:

$$
\begin{aligned}
\kappa & =3 \times 10^{-9} \mathrm{~m}^{2} \mathrm{~s}^{-1} \\
\nu & =10^{-4} \mathrm{~m}^{2} \mathrm{~s}^{-1}
\end{aligned}
$$

Previous experiments on diffusion-driven flow have used stratifications of the order $N \sim 1 \mathrm{~s}^{-1}$. Using a somewhat weaker stratification of $N=0.5 \mathrm{~s}^{-1}$ will further thicken the boundary layer. Combining all of these parameters, we find a boundary layer scale of $\delta=5 \mathrm{~mm}$, where the boundary layer thickness $\delta$ is given in equation (1.12). This gives a full boundary thickness $\delta \pi=16 \mathrm{~mm}$. This should be resolvable with careful use of PIV, as it is about $50 \%$ the thickness of the well-resolved velocity field in Allshouse et al. [2010].

The next challenge is achieving the same parameter range in the lab that was explored in the numerics. The numerics were motivated by oceanic topography, and involve scales of tens of kilometers. In the lab, we probably cannot accurately machine a bottom boundary with corrugation amplitude $A$ much smaller than $2 \mathrm{~mm}$. For convenience when using tanks of common size, we would like our corrugation wavelength $\lambda$ to be on the order of $10 \mathrm{~cm}$. Combining these, we can arrive close to the parameter range explored in the numerical models. Table 2.2 gives some suggested experiments spanning the boundary layer geome- 


\begin{tabular}{ccc|c|ccc|c}
$\alpha$ & $A(\mathrm{~mm})$ & $\lambda(\mathrm{cm})$ & $\delta(\mathrm{mm})$ & $\frac{\Delta h}{A}$ & $\frac{\delta}{A}$ & $\frac{A}{\lambda}$ & Expected Behavior \\
\hline 0.087 & 5 & 10 & 5 & 0.27 & 0.84 & 0.05 & Overflow \\
0.087 & 3 & 10 & 5 & 0.46 & 1.41 & 0.03 & Overflow \\
0.087 & 2 & 10 & 5 & 0.69 & 2.11 & 0.02 & Overflow \\
0.087 & 3 & 25 & 5 & 1.15 & 1.41 & 0.012 & Linear \\
0.087 & 7 & 10 & 3 & 0.20 & 0.43 & 0.07 & Reversing
\end{tabular}

Table 2.2: Proposed topography for laboratory experiments, spanning the range of behavior predicted from the numerics. These points are shown in non-dimensional space in Figure 2-26 as closed circles.

tries observed numerically. These suggested experiments achieve the desired parameter values for $\frac{\Delta h}{A}$ and $\frac{\delta}{A}$, though they have a somewhat larger topographic aspect ratio $\frac{A}{\lambda}$ than the numerical experiments (Figure 2-26). This is probably unavoidable-some adjustment must be made for the laboratory scale.

Because the topographic aspect ratio $\frac{A}{\lambda}$ is significantly larger in these proposed experiments, we must confirm that the hydrostatic approximation would still be valid. The vertical momentum balance is:

$$
\frac{\partial w}{\partial t}+v \frac{\partial w}{\partial y}+w \frac{\partial w}{\partial z}=\underbrace{-\frac{1}{\rho_{0}} \frac{\partial p_{H 0}}{\partial z}-g}_{\text {balance }}-\underbrace{\frac{1}{\rho_{0}} \frac{\partial p_{H^{\prime}}}{\partial z}-g \frac{\rho^{\prime}}{\rho_{0}}}_{\text {balance }}+\frac{1}{\rho_{0}} \frac{\partial p_{D}}{\partial z}+\nu \frac{\partial^{2} w}{\partial y^{2}}+\nu \frac{\partial^{2} w}{\partial z^{2}}
$$

By definition of the hydrostatic pressure terms $p_{H)}$ and $p_{H^{\prime}}$, the pairs of terms indicated by the underbraces balance. Of the remaining terms, the largest are the horizontal advection and vertical viscosity. They compare to the smallest hydrostatic term as follows:

$$
\begin{aligned}
& \frac{v \frac{\partial w}{\partial y}}{-g \frac{\rho^{\prime}}{\rho_{0}}} \sim \frac{A}{2 N \lambda^{2} \alpha \sqrt{\mathbb{P}_{r}}} \sim \frac{1}{40} \\
& \frac{\nu \frac{\partial^{2} w}{\partial z^{2}}}{-g \frac{\rho^{\prime}}{\rho_{0}}} \sim \frac{A \alpha}{4 \lambda} \sim \frac{1}{800}
\end{aligned}
$$

The hydrostatic approximation holds as long as these ratios are small. Even at these larger topographic aspect ratios, they are. In a numerical simulation using a larger value of $\frac{A}{\lambda}$ (Figure 2-27), overflows arise as expected. 
Additionally, note that these larger aspect ratios, we lose a little accuracy with in the approximation that the no-normal-flux bottom boundary condition is about the same as a no-vertical-flux bottom boundary condition, an approximation I routinely make. Instead of being accurate to within $<0.5 \%$, it's accurate to within about $5 \%$ at the steepest sections of slope.

The final challenge in these experiments arises from the expected timescales. The initial establishment of the boundary layer flow happens over a boundary-layer adjustment timescale $T_{B L}$, mentioned in equation (2.2), which is the time it takes for diffusion to act over the thickness of the boundary layer:

$$
T_{B L}=\frac{\delta^{2}}{\kappa}
$$

For the setup I've described in this appendix, that gives an adjustment timescale of about $2-2.5$ hours. To truly reach a steady state, we should probably allow for a few $T_{B L}$ to elapse, so the experiment might take 10-12 hours. This is slow. I am not sure if this is so slow that other effects, like the erosion of the initial stratification, will become significant. We can reduce this time by reducing the boundary layer thickness (through adjusting $N, \nu, \alpha)$, but that will exacerbate the difficulties in visualizing the flow.

In conclusion, it seems plausible that we would be able to create overflow-type behavior in laboratory boundary layers, and to visualize the flow once created. My biggest concern is whether the boundary layers will evolve so slowly that we will not be able to measure a clean effect. 


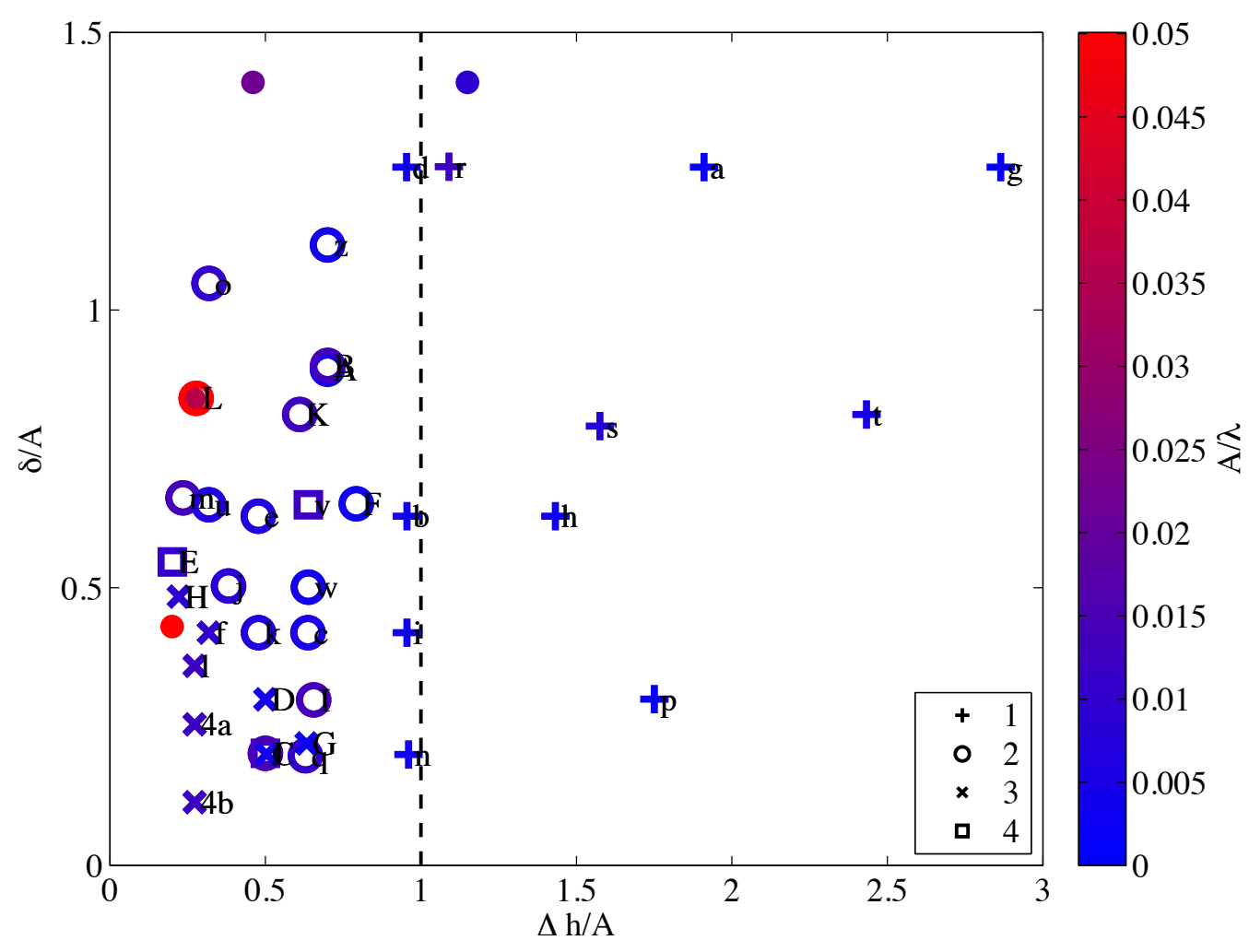

Figure 2-26: The behavior of the boundary layer over a corrugated, sloping bottom, changes as a function of the nondimensional topographic parameters: $\delta / A$, the boundary layer thickness relative to the topographic amplitude; $\Delta h / A$, the vertical separation between corrugations relative to the topographic amplitude; and $A / \lambda$, the aspect ratio of the corrugations. $\Delta h / A>1$ means that the bottom slope is always positive, and there is no trough behind the corrugation. This figure is similar to Figure 2-13, with the color axis adjusted and proposed experiments added. The open circles $(\circ)$ correspond to numerical experiments that show overflows, the crosses $(\times)$ to experiments with reverse boundary layers, and the pluses $(+)$ to linear experiments. The shaded circles $(\bullet)$ correspond to the proposed laboratory experiments from Table 2.2. For this analysis, I performed an additional numerical experiment, labeled $\mathrm{L}$, with a higher value of $A / \lambda$ than the previous experiments, in order to confirm that the overflowing behavior was still predicted. A velocity field from this experiment is shown in Figure 2-27. 


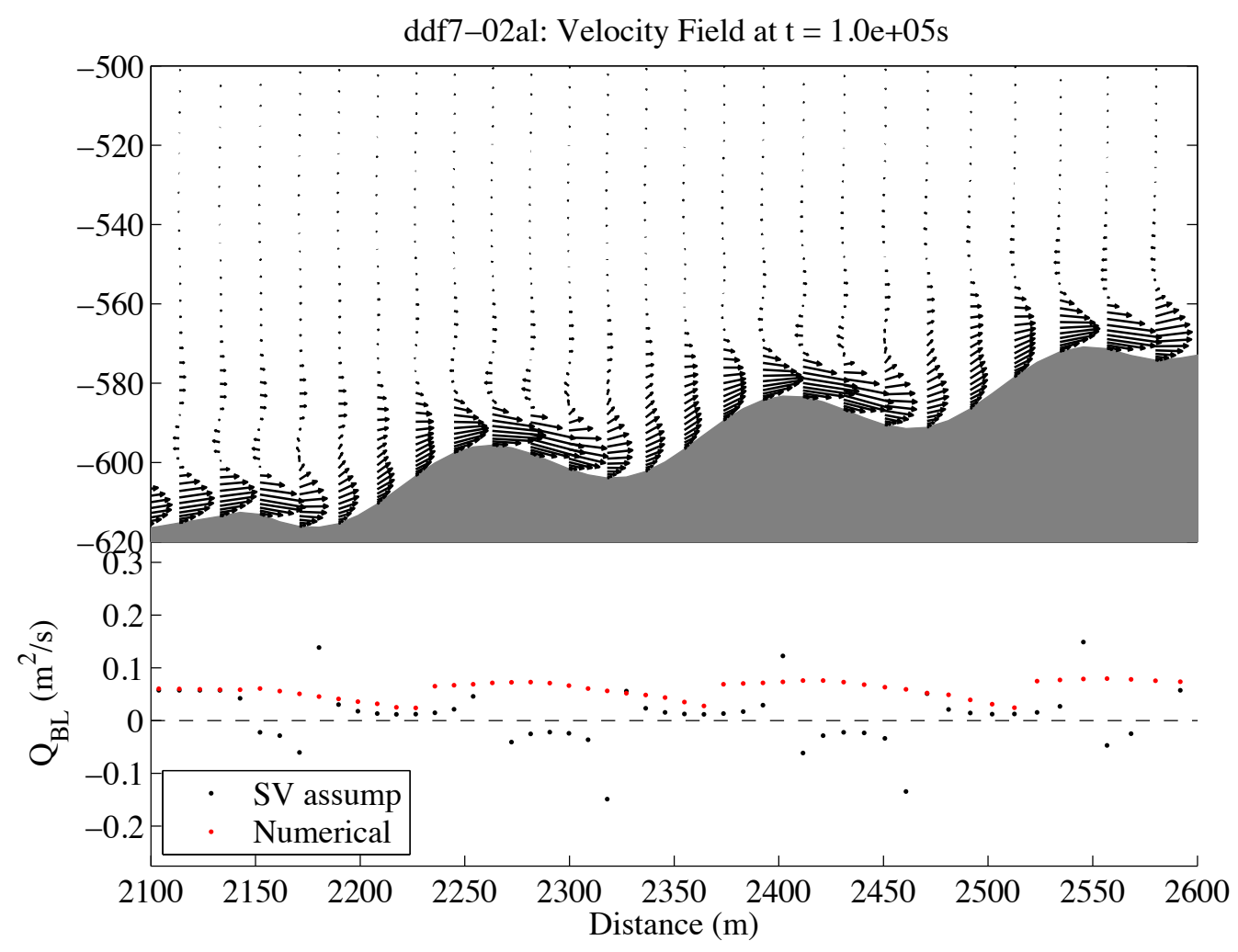

Figure 2-27: We still see overflowing behavior in a numerical experiment with a dramatically higher topographic aspect ratio $A / \lambda$ than previously considered. This experiment uses oceanographic fluid properties $N, \kappa, \nu$, combined with sills that are approximately $7 \mathrm{~m}$ high and $150 \mathrm{~m}$ apart. It is designated as the open circle L in Figure 2-26. 


\section{Chapter 3}

\section{Diffusion-Driven Boundary Layers over Two-Dimensional Topography, with Rotation}

In the previous chapter, we glimpsed of a potential mechanism for abyssal ocean mixing that has previously been neglected. Diffusive, buoyancy-driven boundary layers over varying topography provide a potential mechanism for both increasing mixing in boundary regions and for increasing exchange of water between the boundary and the far field. Boundary layers can both change water properties and communicate those changes to the entire basin. However, in the Chapter 2 I only dealt with non-rotating, two-dimensional flows, so in this chapter we will take a step toward more ocean-like conditions, by introducing rotation while remaining in a two-dimensional frame.

Rotation has a number of consequences beyond simply coupling the upslope buoyancydriven boundary layer to the along-isobath velocity field. That along-isobath velocity drives an Ekman-like boundary layer flow in addition to the buoyancy-driven flux. At steady-state, that Ekman-like boundary layer flow is also upslope. However, since the buoyancy-driven boundary layer responds to the local slope and the rotation-driven boundary layer responds to the average slope, the two effects sometimes reinforce each other 
and sometimes compete. As the rotation rate increases, the boundary layer becomes more Ekman-like, less sensitive to the local topography, and the exchanges between the boundary layer and the far field are suppressed. That is, rotation changes both how the boundary layer affects the water properties and how those effects are communicated to the basin. This happens even though the whole system is ultimately forced by buoyancy and diffusion.

Even though two-dimensional topography is not realistic for the ocean bottom, the interplay between rotation and buoyancy that we explore in this chapter will be essential for understanding the three-dimensional effects we will discuss in the next chapter, and for understanding how these dynamics apply in the real ocean.

\subsection{Introduction}

Rotating, diffusive boundary layers as a potential source of ocean mixing received a significant amount of attention in the 1980s and 1990s. The constant slope (one-dimensional) solution was worked out in some detail.

Early authors in the atmospheric science community worked out the behavior of an Ekman layer over a sloping bottom with a radiating bottom boundary condition, like the Earth's surface warmed by the sun [Holton, 1965, Hsueh, 1969]. This boundary layer behaves very much like a traditional Ekman layer over a flat bottom.

Thorpe [1987] (hereafter referred to as Thorpe) extended the boundary layer described by Phillips by introducing rotation into a steady boundary layer on an infinite uniform insulating boundary. The insulating bottom boundary is crucial, because it is what causes isopycnals to bend and drives the up-slope boundary layer flow in the first place. Thorpe found that the far-field velocity was not an arbitrary forcing, as in an Ekman layer, but was set by the parameters of the boundary layer. There was only one far-field velocity that allowed a steady bottom boundary layer with a dynamical balance between pressure, rotation, and viscosity, After discussing a few other important contributions, I will work through Thorpe's solution, as it is necessary to understanding what happens after we intro- 
duce topography.

Building on Thorpe, Garrett [1990] studied the one-dimensional boundary layer with mixing coefficients that vary in the slope-normal direction. A diffusivity that declines as you move farther from the bottom is probably more like the real ocean, and it induces a downslope flow above the upslope flow in the boundary layer. Garrett is generally skeptical about the efficacy of boundary layer mixing in the ocean, as it is "ineffective in producing significant vertical density flux if the high mixing coefficients occur in regions of low vertical density gradient." That is, the boundary region will become homogenized, so additional mixing will have no further effect on the density field. He acknowledges that changes in the far field stratification could induce exchanges between the boundary region and the far field, but makes no mention of the potential role of changes in the bottom slope.

MacCready and Rhines [1991] introduce time dependence into the boundary layer dynamics. They imagine an arbitrary far field velocity over a constant-slope bottom, and discuss how the initial boundary response is to form an Ekman layer, which is subsequently arrested by buoyant effects. Through a diffusive process, those buoyancy effects are communicated to the far field, slowly adjusting it to the velocity required by Thorpe's steady state solution. This 'arrested Ekman layer' had been previously studied independently from Phillips's and Thorpe's work, because buoyancy effects could significantly alter the dynamically important quantities of the bottom boundary layer, like the Ekman pumping and bottom mixed layer depth. MacCready and Rhines were able to show (as clarified in Garrett et al., 1993) that, at least for constant mixing coefficients, the arrested Ekman layer is the time-dependent adjustment of the boundary layer and interior flow to the steadystate solution of Thorpe. The far-field flow solution specified by Thorpe diffuses into the interior from the boundary layer by a "slow diffusion" equation. Again, however, this work makes no mention of the role of changes in the bottom slope.

To my knowledge, only one previous effort has been made to look at the consequences of a a non-constant insulating slope on the boundary layer. Cummins and Foreman [1998] began to explore the effects of slope curvature and finite slope length with a numerical 
study of the unforced boundary layer over an isolated, axisymmetric Gaussian hill, like a seamount. They observe radially-inward upslope buoyancy-driven boundary layer flows. As the Thorpe solution suggests, they also observe that an azimuthal flow develops, which is geostrophic and on average anticyclonic. Cummins and Foreman also find that the convergence of the boundary layer at the crest of the hill leads to upwelling and to fluid forced out of the boundary region, an effect we saw in the previous chapter that we will see is ubiquitous for these boundary layers.

Now I will review the derivation of the analytical solution from Thorpe and point out some properties of the solution that are important for understanding the effects of topography that we study in the remainder of this chapter. This type of boundary layer is governed by Boussinesq dynamics, and is most usefully described in terms of the same rotated coordinate system we used in the non-rotating work: $x$ is the along-isobath coordinate, $\xi$ is the upslope coordinate, and $\eta$ is the slope-normal coordinate. The velocities in each of these directions are $(u, v, w)$. Occasionally, when discussing the effect of the boundary layer on the far-field, it is useful to use Cartesian coordinates $(x, y, z)$, where $z$ is aligned with the direction of gravity.

We are interested in strictly two-dimensional dynamics in this chapter, so we assume that nothing varies in the along-isobath direction: $\frac{\partial}{\partial x}=0$ for all quantities. Following Phillips, we also assume that the velocities do not vary with $\xi$; the only variations in the upslope $\xi$ direction are the density and pressure decreasing with the uniform background stratification. We assume constant viscosity $\nu$ and diffusivity $\kappa$. Our governing equations are then reduced to:

$$
\begin{aligned}
-\rho_{0} f v \cos \alpha & =\rho_{0} \nu \frac{\partial^{2} u}{\partial \eta^{2}} \\
\rho_{0} f u \cos \alpha & =-\frac{\partial p}{\partial \xi}-\rho g \sin \alpha+\rho_{0} \nu \frac{\partial^{2} v}{\partial \eta^{2}}
\end{aligned}
$$




$$
\begin{aligned}
\rho_{0} f u \sin \alpha & =-\frac{\partial p}{\partial \eta}-\rho g \cos \alpha \\
v \frac{\partial \rho}{\partial \xi} & =\kappa \frac{\partial^{2} \rho}{\partial \eta^{2}}
\end{aligned}
$$

Note that even though the velocity field varies only varies in $\eta$ this problem still requires three momentum equations: the two horizontal equations coupled by rotation, and a vertical equation because the direction of variation $\eta$ does not align with gravity. We express the density as a constant base value, a constant background stratification, and a perturbation $r$ induced by the boundary that depends only on the slope-normal coordinate:

$$
\rho=\rho_{0}-\frac{\rho_{0} N^{2}}{g}(\xi \sin \alpha+\eta \cos \alpha)+\rho_{0} r(\eta)
$$

These equations are subject to the boundary conditions:

$$
\begin{array}{rll}
u, v, \frac{\partial \rho}{\partial \eta}=0 & \text { at } & \eta=0 \\
v, r \rightarrow 0 & \text { as } & \eta \rightarrow \infty \\
u \text { finite } & \text { as } & \eta \rightarrow \infty
\end{array}
$$

Physically, we have no slip and no mass flux at the bottom boundary, no density perturbations to the far field, no velocity with a diapycnal component in the far field, and finite horizontal velocity in the far field. We can then cross-differentiate our $\xi$ and $\eta$ momentum equations, substitute in for $u$ from our $x$ momentum equation, and derive a single equation for $v$, the upslope velocity:

$$
\left(\frac{f^{2} \cos ^{2} \alpha}{\nu^{2}}+\frac{N^{2} \sin ^{2} \alpha}{\kappa \nu}\right) v+v^{\prime \prime \prime \prime}=0
$$

The quantity in the parentheses has units of $L^{-4}$, so we define a boundary-layer scale $\delta_{R}=\frac{1}{\gamma_{R}}$, where $\gamma_{R}$ is:

$$
\gamma_{R}=\left(\frac{f^{2} \cos ^{2} \alpha}{4 \nu^{2}}+\frac{N^{2} \sin ^{2} \alpha}{4 \kappa \nu}\right)^{1 / 4}
$$


Some of the key dynamics of this chapter are embedded in this scale. In the limit where $f \rightarrow 0$, we recover the boundary layer scale $\gamma_{P}$ for the non-rotating Phillips layer discussed in the last chapter. In the limit where $f$ is large, $\gamma_{R} \rightarrow \gamma_{E}=\sqrt{f / 2 \nu}$, the boundary layer scale for a classical Ekman layer. Throughout this chapter, we will see hybrid dynamics between Phillips and Ekman, between buoyancy-driven and rotationally-driven boundary layers, and that is already present here. The structure of the boundary layer thickness $\gamma_{R}$ was chosen such that the the equation for $v$ would become:

$$
v^{\prime \prime \prime \prime}+4 \gamma_{R}^{4} v=0
$$

This is the same equation as Phillips derived in the non-rotating case, but with a boundary layer thickness that has been reduced from the effect of rotation. The solution is:

$$
\begin{aligned}
\rho(\xi, \eta) & =\rho_{0}-\frac{\rho_{0} N^{2}}{g}(\xi \sin \alpha+\eta \cos \alpha)-\rho_{0} \frac{N^{2} \cos \alpha}{\gamma_{R} g} e^{-\gamma_{R} \eta} \cos \left(\gamma_{R} \eta\right) \\
v(\eta) & =2 \kappa \gamma_{R} \cot \alpha e^{-\gamma_{R} \eta} \sin \left(\gamma_{R} \eta\right) \\
u(\eta) & =\frac{f \kappa}{\gamma_{R} \nu} \frac{\cos ^{2} \alpha}{\sin \alpha}\left(1-e^{-\gamma_{R} \eta} \cos \left(\gamma_{R} \eta\right)\right)
\end{aligned}
$$

This solution, shown in for several values of $f$ in Figure 3-1, has a number of interesting properties. First, the upslope velocity and density are identical in structure to the nonrotating case, simply with a reduced boundary layer thickness. The upslope flow carries the same total volume flux as in the non-rotating case, which can be shown by integrating the density equation (3.5) over the full water depth:

$$
Q_{0}=\int_{0}^{\infty} v \mathrm{~d} \eta=\kappa_{\infty} \cot \alpha
$$

$\kappa_{\infty}$ is the diffusivity far from the boundary, though in this work we will consider the case where $\kappa$ is constant. As discussed in the previous chapter on non-rotating boundary layers, this constraint on $Q$ arises from the diffusive density flux in the far field. In a stationary fluid with an insulating bottom boundary, that diffusive flux would cause a flux divergence 

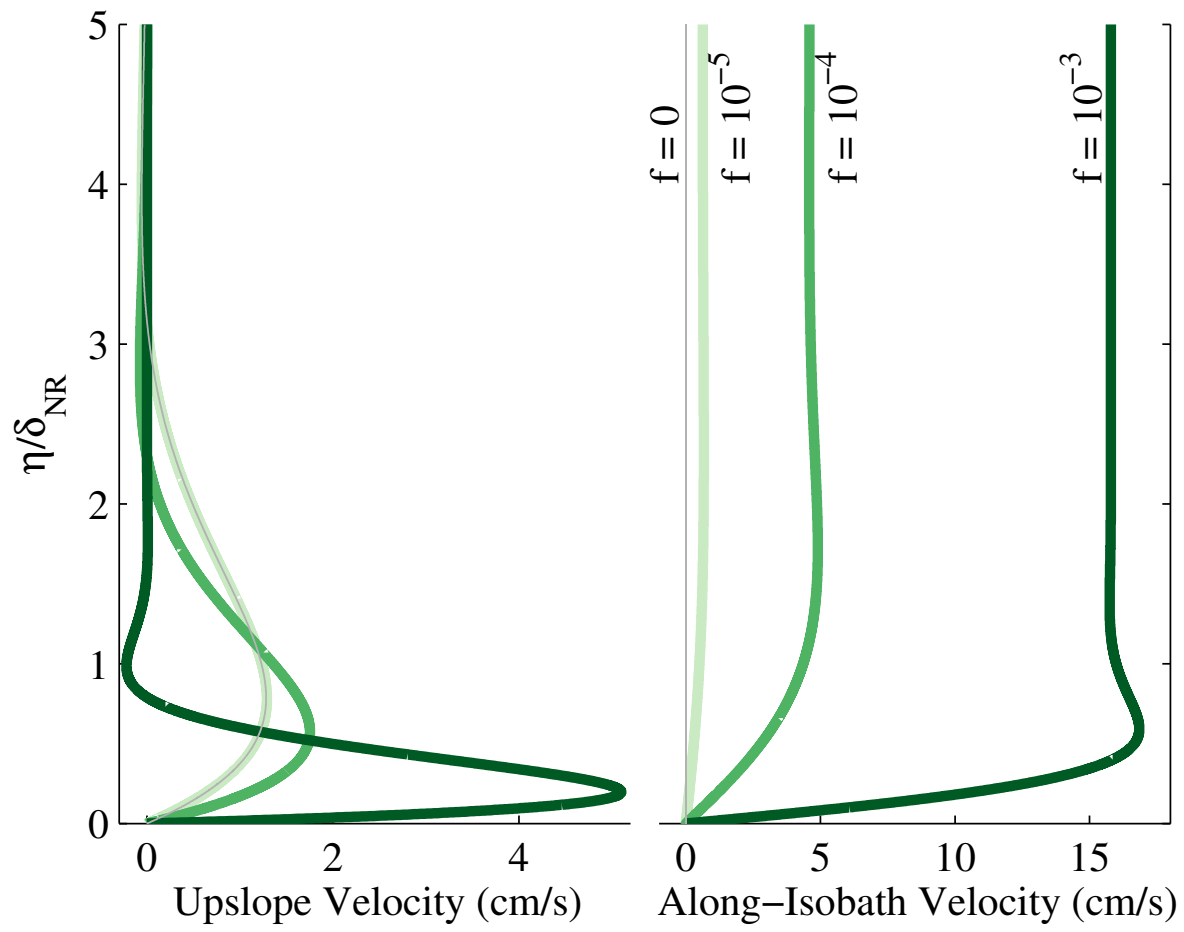

Figure 3-1: As the strength of rotation is increased, the constant-slope analytical solution predicts that the upslope boundary layer velocity becomes more intense and confined to a thinner boundary layer. The thinner, faster rotating boundary layer has exactly the same total upslope volume flux as the non-rotating boundary layer. In the left panel we see the upslope velocity for three values of the Coriolis parameter $f$ in green, and for $f=0$ (thin grey line). At the same time, the along-isobath velocity in the far-field increases with the strength of rotation, shown in the right panel for the same three values of $f$. Throughout this chapter, shades of green will be used to denote rotation strength, with darker green showing stronger rotation.

at the boundary. The advective flux of dense water in the boundary layer must exactly compensate for the diffusive flux in the far field to balance that flux divergence. That advection/diffusion balance is how you maintain a cross-isopycnal velocity in the boundary layer without moving the isopycnals. This thermal condition is equally true regardless of the value of $f$. The effect of this constraint is evident in the upslope velocity; as the boundary layer thins, the peak velocity increases (Figure 3-1).

This thermal constraint also exerts control over the rotating dynamics of the boundary layer. A second interesting property of this solution is that, in addition to having the same 
structure as a buoyancy-driven layer, this boundary layer has exactly the same structure as an Ekman layer for an appropriate along-isobath flow. It simultaneously satisfies the Ekman and the Phillips governing equations. However, to maintain a steady state, the Ekman boundary layer flux must match this thermal constraint. This will only happen for a single value of the along-isobath velocity far from the bottom, called $u_{\infty}$.

This leads directly to the third striking property of this boundary layer: the alongisobath velocity does not decay to zero far from the boundary. Instead, it approaches a constant $u_{\infty}$ that depends on the rotation, bottom slope, and mixing parameters:

$$
u_{\infty}=\frac{f \kappa}{\gamma_{R} \nu} \frac{\cos ^{2} \alpha}{\sin \alpha}=\frac{Q_{0} \delta f \cos \alpha}{\nu}
$$

This particular uniform flow far from the boundary is enforced by the boundary layer. No steady-state solution exists that satisfies both the no-slip bottom boundary condition and has zero velocity in the far field, or any other far-field velocity. Unlike an Ekman layer, we cannot treat the far-field velocity field as an arbitrary forcing-it is set by the boundary properties. As long as the far field velocity is $u_{\infty}$ the buoyant and rotational dynamics hybridize perfectly, and work together to generate the upslope boundary layer current. And, as both Cummins and Foreman [1998] and MacCready and Rhines [1991] observe, if this far-field flow is not initially present, the fluid will adjust to it diffusively. During that adjustment, the boundary layer might experience Ekman layer arrest.

Now that we have reviewed the some of the current understanding of rotating diffusive boundary layers over bottoms with constant slopes, the rest of this chapter will discuss the interaction of of these boundary layers with varying bottom topography. In the next section, I will describe the numerical simulations that I conducted. Following that, in section 3.3 , I will discuss the results of these simulations and a physical understanding of them. The remaining two sections will present some of the difficulties in applying the methods from the previous chapter to predict the geometry of the boundary layer, and discuss conclusions of this work. 


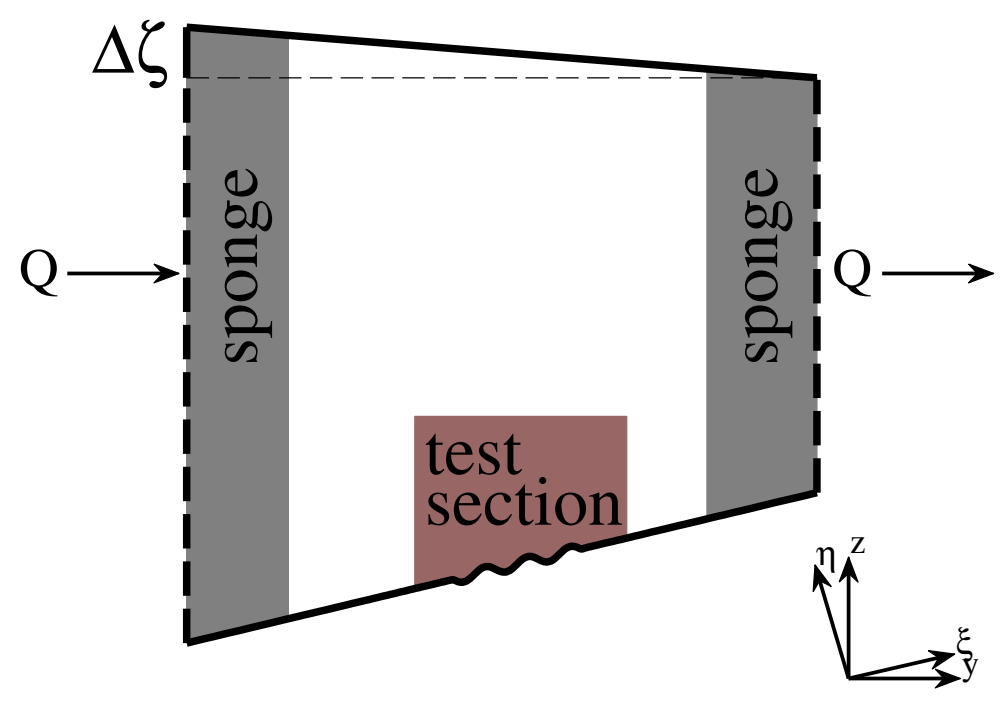

Figure 3-2: In setting up the numerical simulations, I made every effort to insulate the study region of the domain from the effects of the lateral and upper boundaries. This is a typical domain, not to scale. There are open boundary conditions at the upstream and downstream edges, where I specify the depth-integrated mass flux through the domain from equation (4.4). I also specify the change in free-surface height across the domain $\Delta \zeta$, set to maintain a pressure field in geostrophic balance with the far-field along-isobath flow $u_{\infty}$. The results shown in this chapter all come from the test section, a region of varying topography in the middle of the domain separated from the boundaries by long constant slope sections, where the boundary layer matches the analytical solution given in equations (3.10) - (3.12). Sponge layers are used to provide additional insulation of the test section from the boundaries.

\subsection{Numerical Methods}

In most respects, rotating two-dimensional boundary layers can be simulated similarly to the non-rotating two dimensional boundary layers discussed in the previous chapter. Once again, I use the ROMS hydrostatic, primitive equation model [Shchepetkin and McWilliams, 2005], with terrain-following coordinates. I use a prescribed eddy viscosity and diffusivity, $\nu_{e d d y}$ and $\kappa_{e d d y}$, to approximate turbulent mixing processes.

The primary difference between the rotating and non-rotating cases comes from the boundary conditions on the upslope and downslope boundaries, summarized in Figure 3-2. 
The open boundary conditions relevant to the upslope velocity are the same as in the nonrotating case. We expect a net flux of fluid through the domain, and this is accommodated by specifying the depth-averaged velocity through the domain at both the downstream and upstream boundaries. Sponge layers with enhanced horizontal diffusivity at either end of the domain insulate the center of the domain from spurious artifacts that might be introduced by the open boundary conditions. However, in the rotating case, we expect a constant along-isobath velocity far from the boundary, and this must be geostrophically balanced by a barotropic pressure gradient. We therefore specify a difference in free-surface height $\zeta$ between the downstream and upstream boundaries. This height difference is chosen to provide the along-isobath velocity predicted by the constant-slope solution associated with the mean slope. It is:

$$
\Delta \zeta=-\frac{f \Delta y}{g} u_{\infty}
$$

$u_{\infty}$ is the along-isobath flow in the far field given in equation (3.14), and $\Delta y$ is the total size of the domain in the upslope direction. The resulting along-isobath velocity can be dramatically different close to the topography, and often is, but as you move very far from the boundary and the topography, we expect to see only the effect of the average slope. This enforced pressure gradient is necessary to achieving a steady state in the model simulations. Without it we would have to wait for the effects of diffusion to act over the entire water depth—which is nonsensical when you are trying to simulate a semi-infinite fluid. However, as I will discuss in the results, it has a dramatic effect on the boundary layer's behavior.

As in the non-rotating case, the domain has a long constant background slope with a test section of varying topography in the center. The horizontal distance required for the boundary layer to adjust to changes in topography is the adjustment distance $L_{\text {adj }}$ :

$$
L_{a d j}=U_{B L} \times T_{B L}=2 \delta_{R} \cot \alpha
$$

$U_{B L}$ is the upslope velocity scale; $\tau_{B L}$ is the adjustment timescale of the boundary layer, 
a diffusive timescale over the thickness of the boundary layer. The length of the constant slope sections on either side of the test section is typically around $5-10 \times L_{a d j}$. This allows the development of a constant slope boundary layer that we can compare to the analytical solution both before and after the test section. The very close match between the analytical solution and the numerical boundary layer on the constant slope sections gives us confidence that numerical artifacts are not creeping in through the open boundaries. To ensure that the flow behavior remains two-dimensional, the domain is is narrow and periodic in the along-isobath direction.

The simulations here were initiated with $u=u_{\infty}$ and $v, w=0$ throughout the domain and run to what I call a 'dynamically steady state'. This means that I run the numerical simulation until the velocity field reaches a steady state (Figure 3-3), and acceleration term $\frac{\partial u}{\partial t}$ in the momentum balance negligible compared with the pressure, rotation, viscosity, or buoyancy terms. Usually this means the maximum value of the acceleration term is less than $1 \%$ the typical value for the other terms in the momentum balance. Since $u_{\infty}$ is externally enforced, the timescale for the development of a diffusive boundary layer is $T_{B L} \sim \delta^{2} / \kappa$; for the parameter values used in these simulations, that is never more than about 16 hours, and usually much less. Therefore, I typically run the simulation for $2.5 \times 10^{5}$ seconds, or approximately three days.

However, even though the velocity field is stationary, the density field continues to evolve much more slowly. If we were to wait long enough, we would expect the fluid in the troughs between the sills to homogenize completely in cases of weak rotation. In the parameter range we are discussing, we would expect diffusion to take between 100 days and several years to achieve this. The homogenization is further retarded in many cases by the downward vertical advection in the troughs outside of the boundary layer. As discussed by Woods (1991), this advection sharpens the stratification inside the trough. Once enough time has passed to completely homogenize the fluid in the troughs, the boundary layers will break down; though the boundary layer flux is independent of the strength of the stratification, it must be non-zero. In our longest simulations, run for weeks, the stratification 


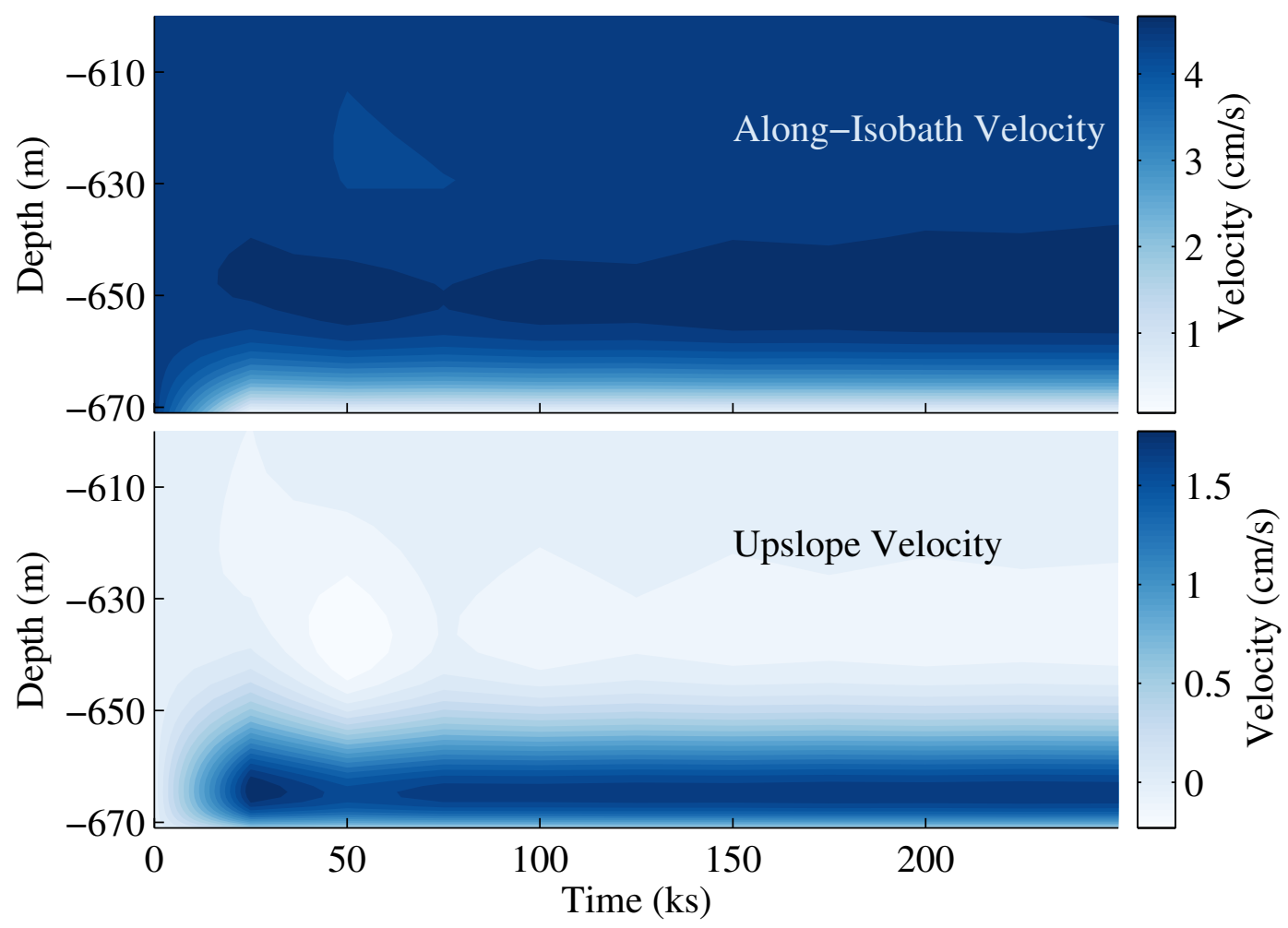

Figure 3-3: The velocity field reached a steady state well before the simulations ended, as the Hovmöller diagrams for the along-isobath and upslope velocities show. The timescale for developing the boundary layer is typically hours, while this simulation was run for almost three days.

within the troughs was still ample to drive a boundary current. We expect that the limit of boundary-layer-driven homogenization in the troughs is not particularly relevant to the real world. Real geophysical fluids all experience external forcings on timescales less than months that would arrest this process. Additionally, real topographies are never strictly two dimensional. Therefore, we confine our attention to the intermediate 'dynamical' steady state that the velocity field reaches on the timescale of hours to a day or so and remains so for weeks or months. 


\subsection{Numerical Results and Interpretation}

The interaction between rotation, buoyancy, and topography gives rise to a variety of interesting boundary layer phenomena. We can gain insight into these boundary layers by thinking about the effects of rotational (Ekman) boundary layers and buoyancy-driven (Phillips) boundary layers separately, and examining the transition between them numerically.

In Chapter 2, we made some progress in understanding one limiting case: how the buoyancy-driven layer interacts with local topography when there is no rotation. Before looking at the numerical results, it's useful to try to understand the other limiting case: how a pure Ekman layer would behave over the same topography. There are two ways that the topography might affect the Ekman layer. First, if we take $u_{\infty}$ as given, the local topography might affect the interaction of the Ekman layer with the far field. Second, the local topography might locally modulate $u_{\infty}$. For a two-dimensional flow, neither of these things happen.

Consider a homogeneous fluid over a bottom with topography $h(x, y)$. Very far from the boundary $(z \rightarrow \infty)$, the fluid has given background velocity $u_{\infty}, v_{\infty}$. In order to satisfy the no-slip and no-normal-flow conditions on $h$, the classical Ekman layer solution has to be modified:

$$
\begin{aligned}
& u=u_{\infty}-e^{-\gamma_{E}(z-h)}\left(u_{\infty} \cos \left(\gamma_{E}(z-h)\right)+v_{\infty} \sin \left(\gamma_{E}(z-h)\right)\right) \\
& v=v_{\infty}-e^{-\gamma_{E}(z-h)}\left(v_{\infty} \cos \left(\gamma_{E}(z-h)\right)-u_{\infty} \sin \left(\gamma_{E}(z-h)\right)\right)
\end{aligned}
$$

Recall that $\gamma_{E}$ is the reciprocal of the traditional Ekman layer thickness. We can see right away that even in the absence of buoyancy effects, topography can modify the Ekman layer. Moreover, those modifications can transmit themselves into the interior. If we use equations (3.17) and (3.18) to calculate the induced vertical velocity far from the boundary, we find:

$$
w_{\infty}=\left(u_{\infty} \frac{\partial h}{\partial x}+v_{\infty} \frac{\partial h}{\partial y}\right)+\frac{1}{2 \gamma_{E}}\left(\frac{\partial v_{\infty}}{\partial x}-\frac{\partial u_{\infty}}{\partial y}\right)
$$


The term in the right parentheses have the Ekman pumping absent topography, while the term in the left parentheses are an additional term that arises from satisfying the nonormal-flow condition at the locally sloping bottom boundary. In the present two-dimensional case, however, $\frac{\partial}{\partial x}=0$ and $v_{\infty}=0$, which implies that $w_{\infty}=0$. The topography has no effect on the way that the Ekman layer interacts with the far-field. The Ekman layer is entirely determined my $u_{\infty}$ and produces no pumping out of the boundary layer.

It is also impossible for the local topography to affect the Ekman layer by modulating $u_{\infty}$. Since the buoyant effects drive an along-isobath velocity that extends truly infinitely far from the boundary, there is no such thing as "slowly-varying" topography for the purposes of $u_{\infty}$. When viewed from sufficiently high, all hills look small, so $u_{\infty}$ must correspond to their mean effect. To satisfy mass conservation and maintain the advectivediffusive balance in the boundary layer, we need $u_{\infty}$ to be such that the upslope Ekman boundary layer flux matches the average buoyancy-driven upslope flux. This relationship clarifies the expression for $u_{\infty}$ for a constant slope given in equation (3.14). In a classical Ekman layer, the boundary layer flux perpendicular to $u_{\infty}$ is $Q=2 u_{\infty} / \gamma_{E}$, and equation (3.14) reduces to this if we neglect buoyant effects. In summary, local topography does not impress itself on the far-field through the Ekman layer. The Ekman layer only responds to $u_{\infty}$.

Now that we have some understanding of how we expect the boundary layer to behave both in the limit of weak rotation and in the limit of strong rotation, we can examine the transition between those two end states using numerical simulations. To do this, it first helps to have a way to compare the size of the rotational and buoyancy-driven effects. A convenient metric of rotation strength is the slope Burger number:

$$
S=\frac{N \sin \alpha}{f \cos \alpha} \sim \frac{\text { Buoyancy }}{\text { Rotation }}
$$

As $S \rightarrow 0$, rotational effects dominate, and as $S \rightarrow \infty$, we return to the non-rotating dynamics described in the previous chapter. The reciprocal boundary layer thickness $\gamma_{R}$ 
given in equation (4.2) then becomes:

$$
\gamma_{R}=\gamma_{E}\left(1+S^{2} \mathbb{P}_{r}\right)^{1 / 4} \sqrt{\cos \alpha}
$$

$\gamma_{E}$ is the reciprocal Ekman layer thickness $\sqrt{f / 2 \nu} \cdot \mathbb{P}_{r}$ is the Prandtl number $\frac{\nu}{\kappa}$, giving the relative strength of viscosity and diffusion.

As we just discussed, in the strong-rotation limit $(S \rightarrow 0)$, we have a boundary layer that is insensitive to local topography. Given a uniform far-field flow $\left(u_{\infty}\right)$ we expect a uniform upslope Ekman flux. In the weak rotation limit $(S \rightarrow \infty)$, we return to the boundary layer described in the last chapter, driven by diffusive and buoyant effects and strongly influenced by varying topography. This means that with intermediate values of $S$, the rotational effects and buoyant effects may reinforce or cancel each other, depending on topography.

We can clarify the interplay between rotation and diffusion by looking at the terms in the momentum balance for a boundary layer over a rotating two dimensional slope (Figure 3-4). As we can see from the governing equations (3.1) - (3.5), far from the boundary the dynamics are geostrophic. A constant along-isobath velocity throughout the farfield is balanced by the pressure gradient induced by the free-surface slope. Closer to the boundary but outside of the viscous boundary layer, changes to the density field can cause local changes to the pressure gradient and so to the along-isobath velocity. The vertical scale of these changes is approximately given by the horizontal scale of the topography $\lambda$ multiplied by the Prandtl ratio $\frac{N}{f}$, so in these simulations we generally find vertical scales of hundreds of meters or more. However, the effects are weak. In the case illustrated, where $f=10^{-4} \mathrm{~s}^{-1}$ and $S=0.6$, these changes are no more than $10 \%$ the undisturbed background values. Only when we get within the boundary layer itself, less than $\pi \delta_{R}$ off the bottom boundary, is the upslope momentum balance between pressure and rotation modified to include viscous dissipation.

The along-isobath momentum balance, shown in the same figure, is also straightforward. The constant along-isobath velocity $u_{\infty}$ is brought to zero in the bottom boundary 
layer through viscous effects. These are rotationally balanced by an implied upslope velocity. As in the non-rotating case, the horizontal advection terms can become significant in the vicinity of sills. However, the Reynolds number remains small and the primary momentum balance remains between viscosity and rotation.

We can see through the momentum balance that, unlike the non-rotating case, we now have two mechanisms driving the boundary layer: buoyant effects, seen in the pressure gradient term, and rotational effects. With the introduction of varying topography, these two mechanisms - buoyant and rotational—can locally reinforce or compete with each other. In the coordinates used in this chapter, the rotational Ekman flux is always in the positive $\xi$ direction, up the average slope. The buoyancy-driven flux is always up the local slope. If the topography is tall enough to reverse the local slope, as in Figure 3-4, the buoyant flux might be in the opposite direction from the rotationally-driven flux.

The consequences of these competing effects appear in the upslope velocity field for the same topography but with several different values of Coriolis parameter $f$ (Figure 3-5). We begin with very weak rotation, $f=10^{-5} \mathrm{~s}^{-1}$ and $S=6$ (top row). The $(\xi, \eta)$ velocity field is essentially identical to the non-rotating case, which is reassuring. There is a stronger upslope boundary layer flow on the upstream (left) side of each sill, and a weak upslope boundary layer flow on the downstream (right) side of the sill with a recirculation within each trough.

As the strength of rotation is increased to $f=5 \cdot 10^{-5} \mathrm{~s}^{-1}$ and $f=10^{-4} \mathrm{~s}^{-1}$ (middle row), this recirculation becomes smaller and then disappears, moving the boundary layer from a reversing-type geometry to an overflow-type geometry. This is contrary to what

Figure 3-4 (facing page): The numerically calculated momentum terms in both the upslope and along-isobath directions clearly show a balance between pressure, rotation, and viscous dissipation. The upper block of six frames shows the acceleration, pressure gradient, advection, viscosity, and Coriolis terms in the upslope $\xi$ direction for a rotating model run to steady state. The pressure field, generated by a free-surface slope, is nearly uniform, with perturbations induced by the topography. The along-isobath momentum balance, shown in the lower block of six panels, is between viscosity and rotation. In this example, $f=10^{-4} \mathrm{~s}^{-1}$ and $S=0.6$. 

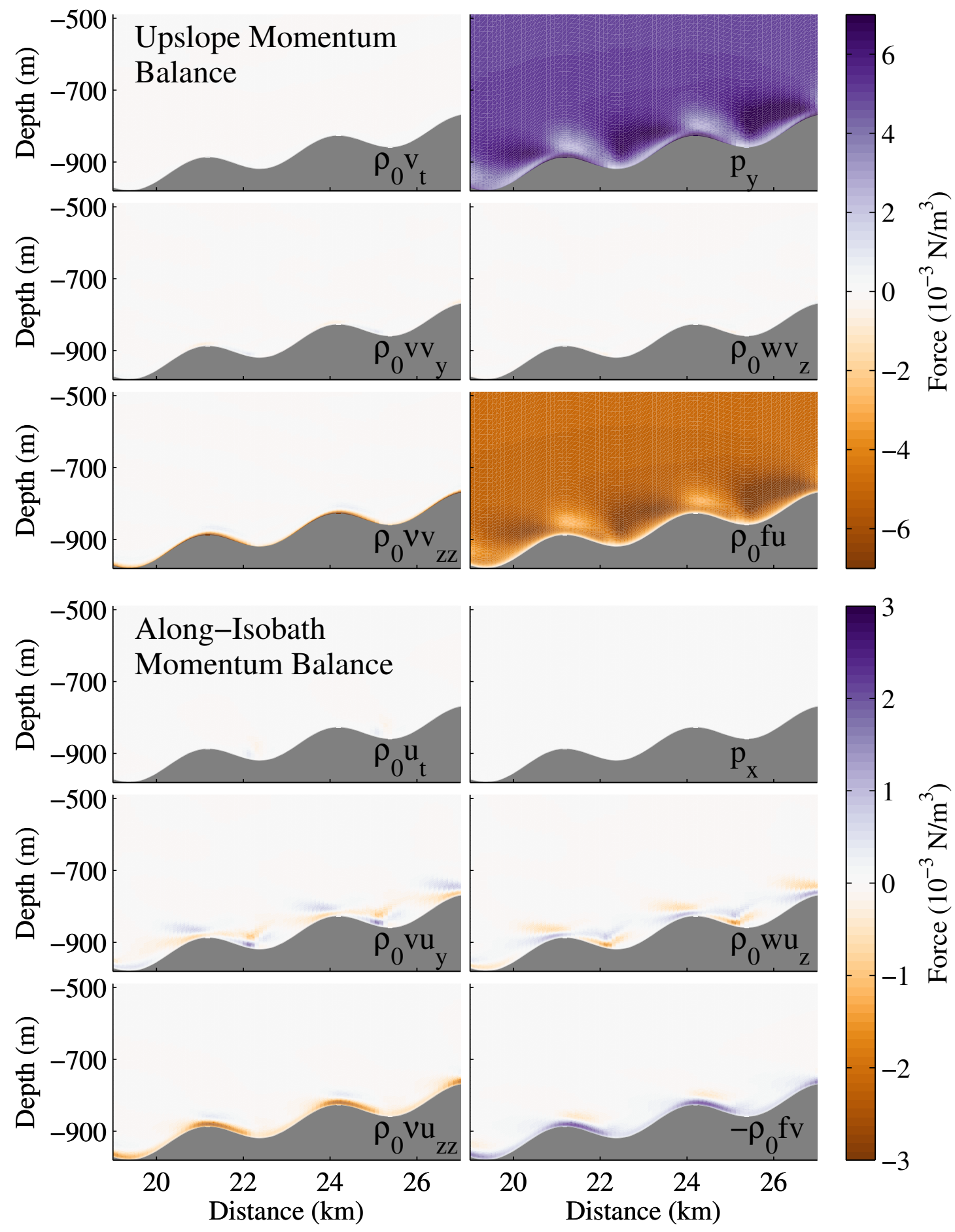

[hp] 


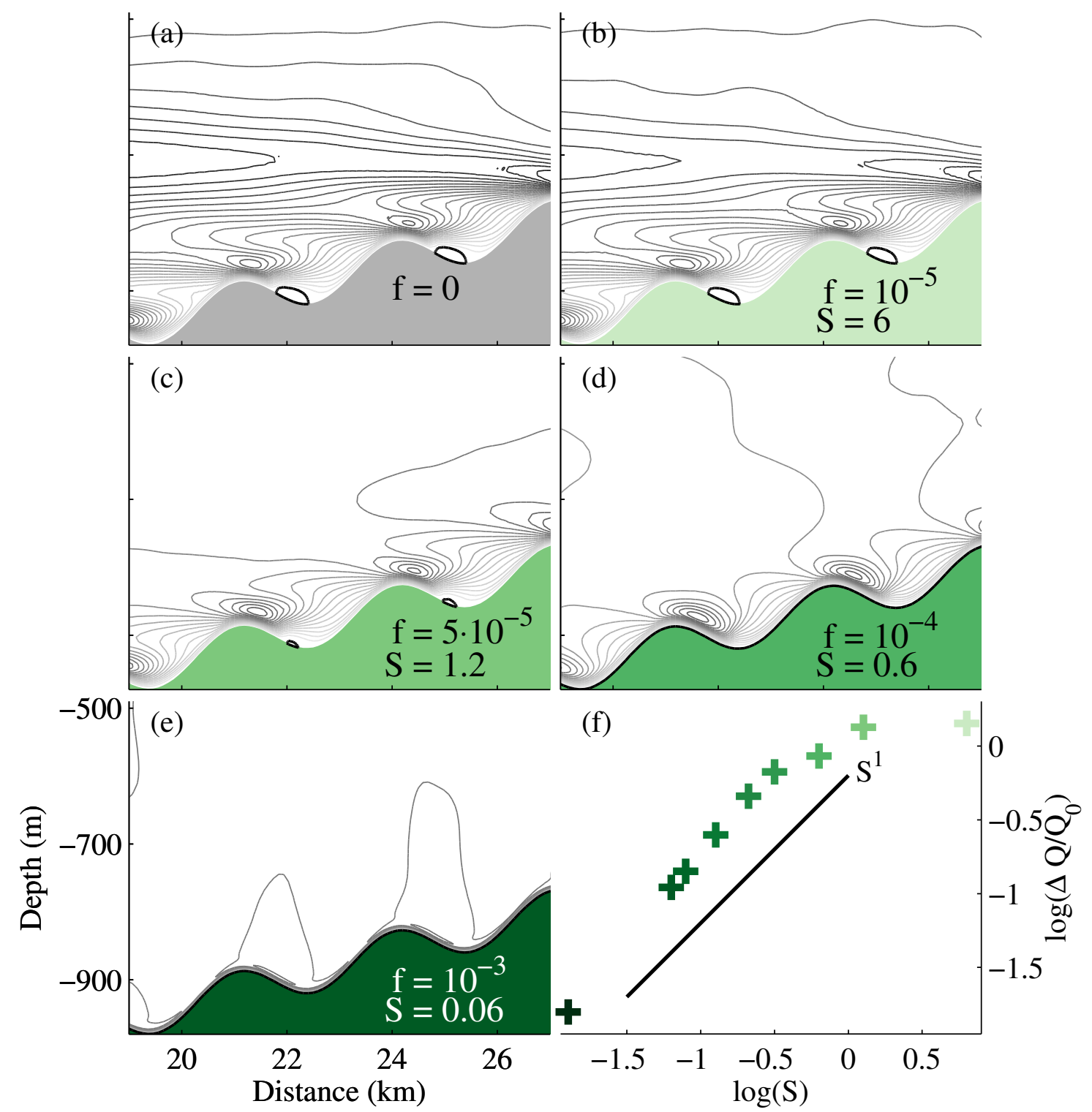

Figure 3-5: As the strength of rotation is increased, the bottom boundary layer behaves more and more like an Ekman layer. The subplots show numerically calculated $(\xi, \eta)$ streamfunctions for five different values of the Coriolis parameter $f$ over identical topography. The thick black contour is $\psi=0$. As $f$ increases, the uniform Ekman boundary layer driven by the uniform far-field flow becomes more and more dominant, with less and less importance for topographically-induced buoyant boundary layer effects. This suppresses exchange of fluid between the boundary region and the far field. The bottom right subplot shows the normalized volume of fluid exchanged as a function of the slope Burger number $S$. 
we would expect from the flow geometry prediction of the previous chapter's discussion of non-rotating boundary layers. As the rotation increases and the boundary layer thins, the horizontal distance $L_{a d j}$ needed for it to adjust to its constant slope behavior becomes shorter. We therefore expect the boundary layer to be more sensitive to the local slope than to the effects of the mean background slope. That would imply upslope flows on both sides of the sills for larger $f$. This can be seen in the regime diagram we used to synthesize boundary layer behavior in the last chapter (Figure 3-6): increasing rotation causes the boundary layer to thin, moving the simulation vertically downward on the diagram and toward the region where we expect to see reversing boundary layers. Why do our expectations based on the non-rotating boundary layer fail?

We neglected to include the rotationally-driven component of the boundary layer flow, which responds to the average topography and is always in the positive $\xi$ direction. As the rate of rotation grows, this component becomes larger and eventually overpowers the buoyant effects on the downstream side of the sill, causing $v>0$ everywhere in the boundary layer. However, at these intermediate rotation strengths $-S=0.6$ and $S=1.2$ - the effects of buoyancy and topography are still clear in the flow recirculations above the boundary layer in the lee of each sill and the along-stream variations in boundary layer volume flux $Q$.

Continuing with our progression through increasing rate of rotation (Figure 3-5), we finally come to the rotationally-dominated boundary layer in the bottom left corner, with $f=10^{-3} \mathrm{~s}^{-1}$ and $S=0.06$. Here, the boundary layer behaves almost purely like an Ekman layer, with little along-stream variation in boundary layer flux. Since the buoyancy effects have been entirely overpowered, it is insensitive to the local topography.

The rate at which the buoyant effects are overpowered by the rotational effects can be estimated by comparing the velocity scale of a buoyancy-driven (Phillips) boundary layer $v_{P}$ to the velocity scale of the rotational (Ekman) boundary layer $v_{E}$ :

$$
\frac{v_{E}}{v_{P}} \sim \frac{f Q_{0} \delta_{E} \cos \alpha / \nu}{2 Q_{0} / \delta_{P}} \sim \frac{\delta_{P}}{\delta_{E}}
$$




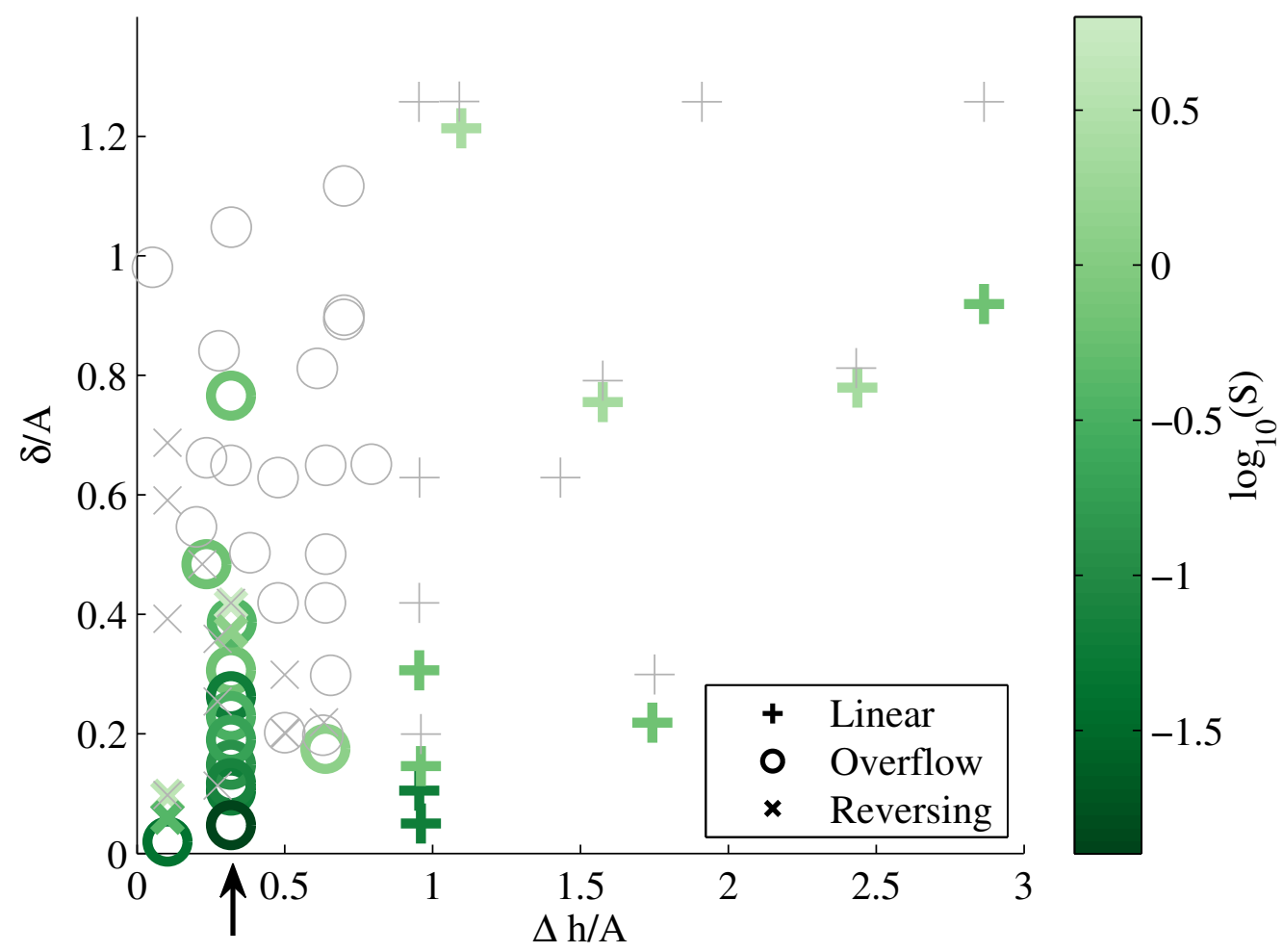

Figure 3-6: As in the non-rotating case, we can map the flow geometry from the numerics in terms of a non-dimensional description of the topography. Following Figure 3-1, the shade of green indicates the value of $f$ for each simulation, with light gray markers for no rotation. Unlike the non-rotating case, we see many overflows in simulations with small values of $\delta / \mathrm{A}$, where the boundary layer is much thinner than the topography amplitude. The small arrow indicates the series of numerical experiments shown in Figure 3-5.

where $\delta_{E}, \delta_{P}$ are the boundary layer thicknesses for the Ekman and Phillips layers, respectively, and $Q_{0}$ is the total boundary layer flux associated with the mean slope, from equation (4.4). This shows that the relative strengths of rotation and buoyancy can once again be captured by the slope Burger number: $\frac{v_{E}}{v_{P}} \sim\left(S^{2} \mathbb{P}_{r}\right)^{1 / 2} \sim S$. This is dramatically confirmed by the amount of fluid exchanged between the boundary region and the far-field.

One of the key results of the previous chapter was that topography can force exchanges between the boundary region and the far field, allowing boundary layer processes to either 


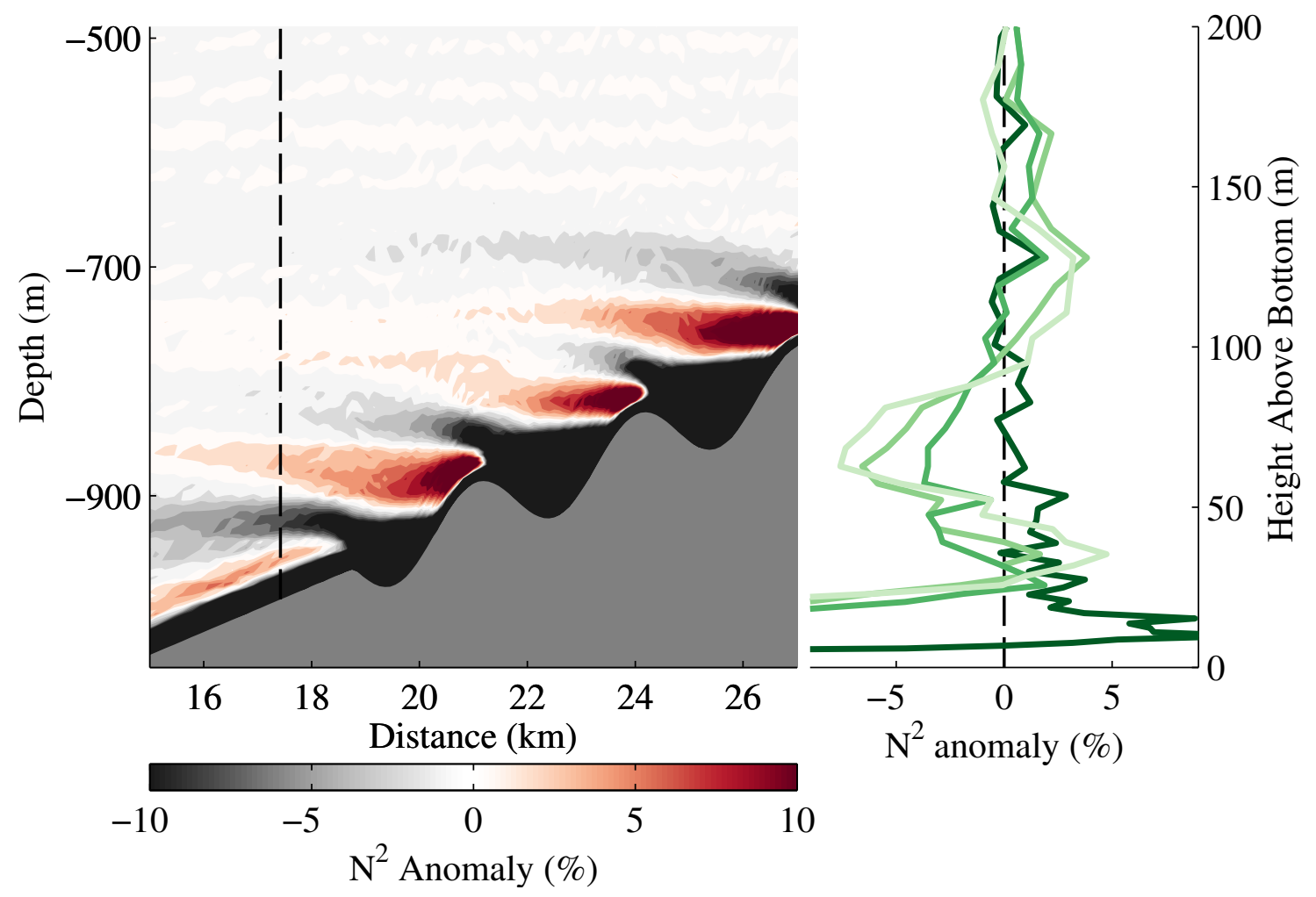

Figure 3-7: Rotating boundary layers can force significant changes to the far-field stratification, though these effects are reduced as the rate of rotation is increased. On the left, we see that after only three days a weakly rotating boundary layer can change the stratification kilometers away from the boundary by more than 5\%. Though the velocity field has reached a steady state in these simulations, the density field continues to evolve, so we expect these anomalies to continue to grow with time. On the right, we see sections of $N^{2}$ taken at the black dashed line for this topography and four different values of $f$ from $10^{-5}$ to $10^{-3} \mathrm{~s}^{-1}$. As rotation increases (going from light to dark green), the effect of the topography on the far field stratification declines until it is negligible.

sharpen or reduce stratification far away from the bottom. However, we see that rotation reduces the effectiveness of this process (Figure 3-5, bottom right) because the rotational part of the boundary layer does not respond to local topography. We can estimate the amount of exchange between the boundary layer and the far field by taking the range of volume fluxes carried in the boundary layer. This range, and the far-field exchanges it implies, can be larger than the mean flux associated with the mean slope, $Q_{0}$, especially for topographies where the volume flux is negative in some places (i.e. the boundary layer flow has reversed direction). As rotation increases, this range decreases, until we reach 
the rotationally-dominated case with almost no exchange. The rate at which $v_{E}$ comes to dominate $v_{P}$ is the same rate at which the volume exchanged $(\Delta Q)$ approaches zero, its value for a pure Ekman layer. This scaling remains accurate over values of $f$ spanning two orders of magnitude.

This relationship between rotation and variations in boundary layer flux can be seen directly in the stratification kilometers away and hundreds of meters above the topography (Figure 3-7). When rotation is weak or moderate, layers of locally increased or decreased stratification form at the depths where the boundary layer is either entraining fluid or forcing fluid out. These stratification anomalies propagate away from the topography. In the case shown, we see that after less than three days $N^{2}$ has been increased or decreased by more than 5\% more than $3 \mathrm{~km}$ from the nearest sill. However, these anomalies are absent in the simulation where $f=10^{-3} \mathrm{~s}^{-1}$. This is consistent with the Taylor-Proudman theorem that increased rotation causes increased vertical coherence in fluid motion. Since these simulations are run to a dynamical steady state, the density field is expected to continue to evolve slowly with time. In the real ocean, these effects on the far-field stratification will continue to grow with time until they are arrested by another process, not included in my numerical simulations.

The local competition between buoyant and rotational forcing can create some counterintuitive results for the density field close to the topography as well. In the constant-slope boundary layer, the boundary layer current carries dense water up the slope at exactly the same rate that diffusion transforms it into lighter water, allowing a continuous vertical velocity with no associated vertical motion of the isopycnals. However, introducing topography can upset this balance. Even though the whole boundary layer system is ultimately driven by buoyant effects, the rotational effects can locally dominate the boundary layer. For example, the buoyancy-driven velocity at a sill might be quite small or even zero if there is no overflow. At the same time, the uniform rotationally-driven velocity might be substantial. This causes the boundary layer to advect dense water faster than it can be balanced by diffusion, carrying the isopycnals downstream and spreading them out parallel to 


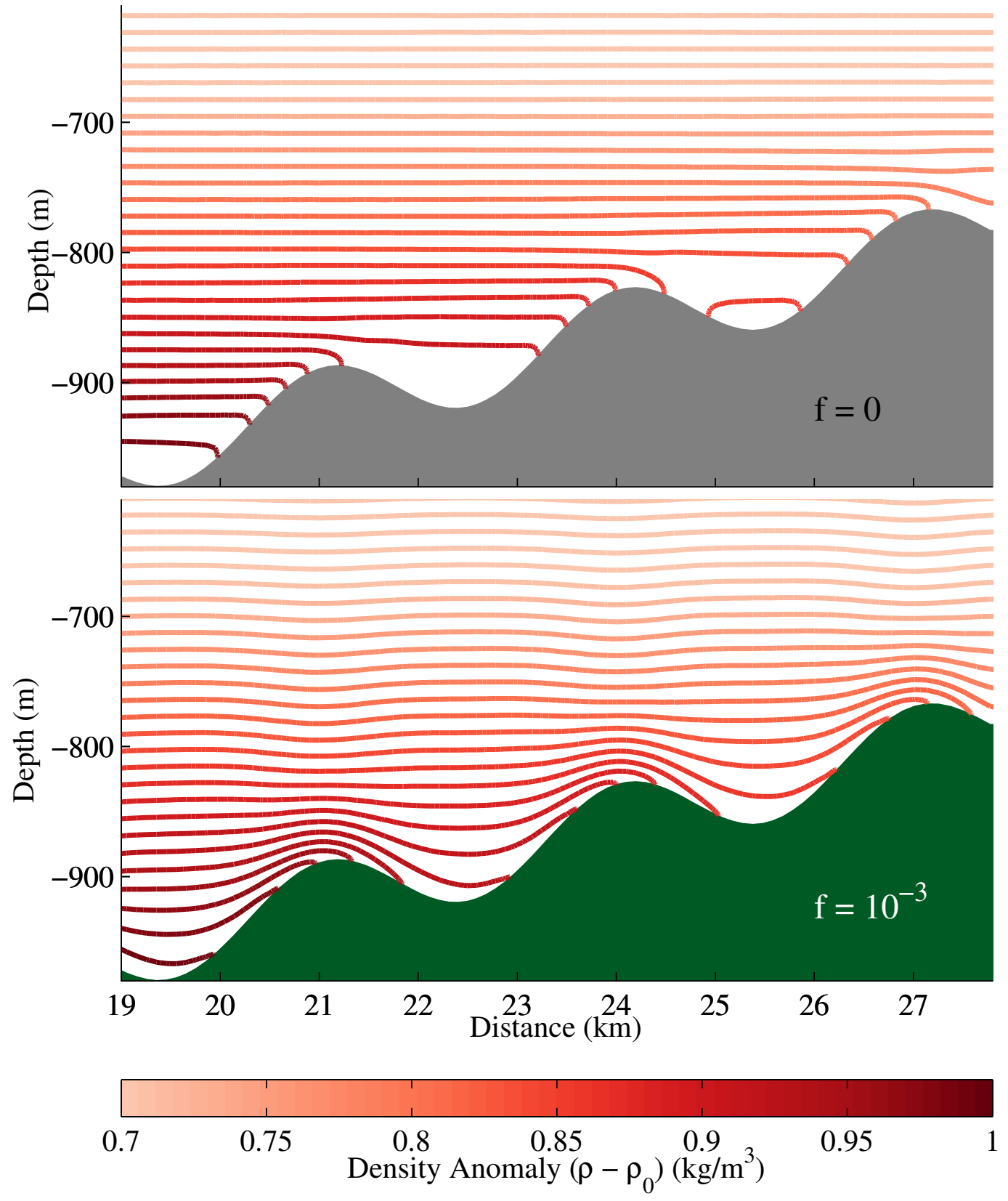

Figure 3-8: At steady-state, the rotational effects can dominate the buoyancy effects, causing buoyancy advection in the boundary layer. When the rotation is strong, the isopycnals are dragged out parallel to the topography, as shown in the upper figure. The Ekman boundary layer velocity is set by the far-field flow, which is set by the mean slope, not the local topography. If rotation is strong, that Ekman velocity may be much larger than the velocity induced by buoyancy effects in the local topography. 
the bottom (Figure 3-8). As this process makes the stratification sharper, local diffusion increases, and eventually a new steady state can be reached.

However, as we saw in Figure 3-8, this process can change the curvature of the isopycnals between two sills. With weak or no rotation, the isopycnals bend downward toward the bottom in a trough, while with strong rotation they follow the topography in bending upward. This has a dramatic effect on the along-isobath velocity (in and out of the page), especially in the region that is outside the viscous boundary layer but close enough to the bottom to feel topography, with a height off-bottom greater than $\pi \delta_{R}$ but less than $f \lambda / N$. In this region, we can approximate the along-isobath velocity $u$ using a thermal wind relation:

$$
g \frac{\partial \rho}{\partial y}=\rho_{0} f \frac{\partial u}{\partial z}
$$

Since we are looking outside the boundary layer, it is more convenient to use gravitationallyaligned coordinates $(y, z)$ instead of slope-normal coordinates $(\xi, \eta)$. The horizontal gradient in density is proportional to the vertical shear in along-isobath velocity. If we look in a trough between two sills, we see that when we go from weak rotation to strong rotation, the sign of that density gradient changes everywhere as the isopycnals move from bending downward to bending upward. This means that the along-isobath velocity induced by the variations in topography also switches direction, from an anti-cyclonic circulation around the trough for weak rotation to a cyclonic circulation for strong rotation.

In this section we have seen how the presence of rotation influences boundary layers over varying topography. Many of the most interesting dynamics arise because buoyant effects respond to the local slope, while rotational effects respond to the far-field alongisobath velocity, set by the average slope. This means that the consequences of changes in topography - that is of the local slope being different than the mean slope-depend strongly on the value of slope Burger number $S$, the ratio between the size of buoyant and rotational effects. Where $S$ is large, the buoyant effects dominate, returning us to the nonrotating dynamics of the last chapter. Where $S$ is small, the boundary layer becomes more and more like an Ekman layer, with a continuous flow up the mean slope. This suppresses 
exchange with the far-field, so strongly rotating boundary layers have a smaller impact on the far-field stratification.

In the last chapter, we were able to describe the basic geometry of our boundary layers as linear, reversing, and overflow, and found that we could predict the behavior for a given topography by estimating the bottom stress at the sill. Those descriptive terms still apply for rotating boundary layers, though their distribution in our parameter space (Figure 3-6) is very different than for non-rotating boundary layers. We would like to know if the bottom stress can still be used to make useful predictions about boundary layer behavior. I will try to answer that question in the next section.

\subsection{Predicting Flow Geometry with Bottom Stress}

In the previous chapter, we were able to use estimates of bottom stress at a sill to predict what topography would produce linear boundary layers, overflows, or reversing boundary layers, even without numerically simulating the flow over a given topography. We would like to use similar arguments to predict the behavior of rotating boundary layers. However, extending the bottom stress argument of the non-rotating work is not trivial. This approach consistently overestimates the influence of rotation. Even for very weak rotation, where we see little effect of rotation in the full numerical simulations (for example as shown in Figure 3-5), this approach predicts that the rotational effects will dominate.

This approach rests on approximating the full rotating diffusive boundary layer described in the introduction to this chapter as a superposition of a diffusive (Phillips) boundary layer and a rotating (Ekman) layer, as we did to understand the results in the previous section. This approximation is asymptotically correct for both $f \rightarrow 0$ and $f \rightarrow \infty$.

In the non-rotating case, we used the upslope boundary layer velocity to calculate a bottom stress, and compared the predicted bottom stress from the upstream and downstream face of each sill to estimate the bottom stress at the sill. In the rotating case, it is unfortunately not possible to simply apply the same procedure using the modified velocity 
in equation (3.12). As we saw in the last section, the buoyancy-driven part of the boundary layer is always oriented up the local slope, but the rotationally-driven part is always to the right of the uniform far-field flow. Rotation therefore accelerates the boundary layer on the upstream side of a sill and retards or reverses the boundary layer on the downstream side. As the strength of rotation is increased, overflows become more likely and the whole boundary layer behaves more like an Ekman layer (Figure 3-5).

To address this asymmetry, I separate the rotating (Ekman) from the diffusive (Phillips) contributions to the bottom stress at the sill. In the non-rotating case, I approximated the bottom stress at the sill as:

$$
\text { Estimated Bottom Stress }=\left.\nu \frac{\partial}{\partial z}\left(v_{P 1}\left(\xi_{\text {sill }}\right)-v_{P 2}\left(\xi_{\text {sill }}\right)\right)\right|_{z=\text { bottom }}
$$

where $v_{P 1}$ is the expected Phillips boundary layer velocity from the upstream slope of the sill and $v_{P 2}$ is the expected Phillips boundary layer velocity from the downstream slope of the sill. In the rotating case, I add a third term, from the Ekman layer velocity arising from the far-field pressure gradient:

$$
\text { Estimated Bottom Stress }=\left.\nu \frac{\partial}{\partial z}\left(v_{P 1}\left(\xi_{\text {sill }}\right)-v_{P 2}\left(\xi_{\text {sill }}\right)+v_{E}\right)\right|_{z=b o t t o m}
$$

There is no need to express $v_{E}$ as a function of location, as it is uniform. For clarity, I refer to these three terms as $\tau_{P 1}$, the bottom stress from the Phillips layer on the upstream slope (calculated as in chapter 2); $\tau_{P 2}$, the bottom stress from the Phillips layer on the downstream slope; and $\tau_{E}$, the bottom stress from the Ekman layer. Then, I once again predict that where $\tau<0$, the stagnation point will be upstream of the sill, giving a reversing boundary layer, and where $\tau>0$, the stagnation point is downstream of the sill, giving an overflow. The total bottom stress is estimated as: $\tau_{P 1}+\tau_{P 2}+\tau_{E}$, superimposing the rotational and buoyant effects. Figure 3-9 shows $\tau_{P 1}+\tau_{P 2}$ in the upper left panel, $\tau_{E}$ in the upper right panel, and their sum in the lower panel with the results of numerical simulations overlaid. The grey contour separates the predicted overflows (above) from the predicted 
reversing boundary layers (below) when $f=0$. The very small light green contour that terminates at 0.5 on the $\mathrm{x}$-axis is the analogous prediction for $f=10^{-5} \mathrm{~s}^{-1}$.

This approach overestimates the effect of rotation when compared to the full ROMS numerical simulation or the analytical solution. Introducing weak rotation $\left(f=10^{-5} \mathrm{~s}^{-1}\right)$ has almost no effect on the upslope velocity field in the analytical solution (Figure 3-1) or in the ROMS simulation (Figure 3-5). However, even this small amount of rotation dramatically changes the prediction for flow behavior in the non-dimensional topography regime diagram (Figure 3-9). It essentially eliminates the area in parameter space where we expect to see a reversing boundary layer, that is upslope boundary layer flows on both sides of a sill.

To understand this discrepancy, it is worth recalling the conditions that generated reversing boundary layers with no rotation. In the non-rotating case, we expect to see a reversing boundary layer when two things are true:

1. The topography is much taller than the boundary layer thickness, allowing enough scale-separation between the boundary layer and the topography for the boundary layer to adjust to close to its constant-slope behavior (that is, the topography should be close to the bottom of Figure 3-9); and

2. The background slope is weak, so each sill is relatively symmetric upstream and downstream (that is, the topography should be close to the left of Figure 3-9).

Together these two conditions mean that while the bottom stress from the boundary layers on each side of the sill is substantial, the two sides cancel almost perfectly at the sill. This prevented an overflow from forming. To this situation, we add an Ekman layer with a bottom stress that is always positive. Even a relatively small Ekman bottom stress can have a dominant effect once the buoyancy-driven flows cancel one another out. And it always encourages the boundary layer to form an overflow. We can see this in the dramatic difference between the upper left panel and the lower panel of Figure 3-9.

However, it doesn't explain why this effect should seem so much more important when 

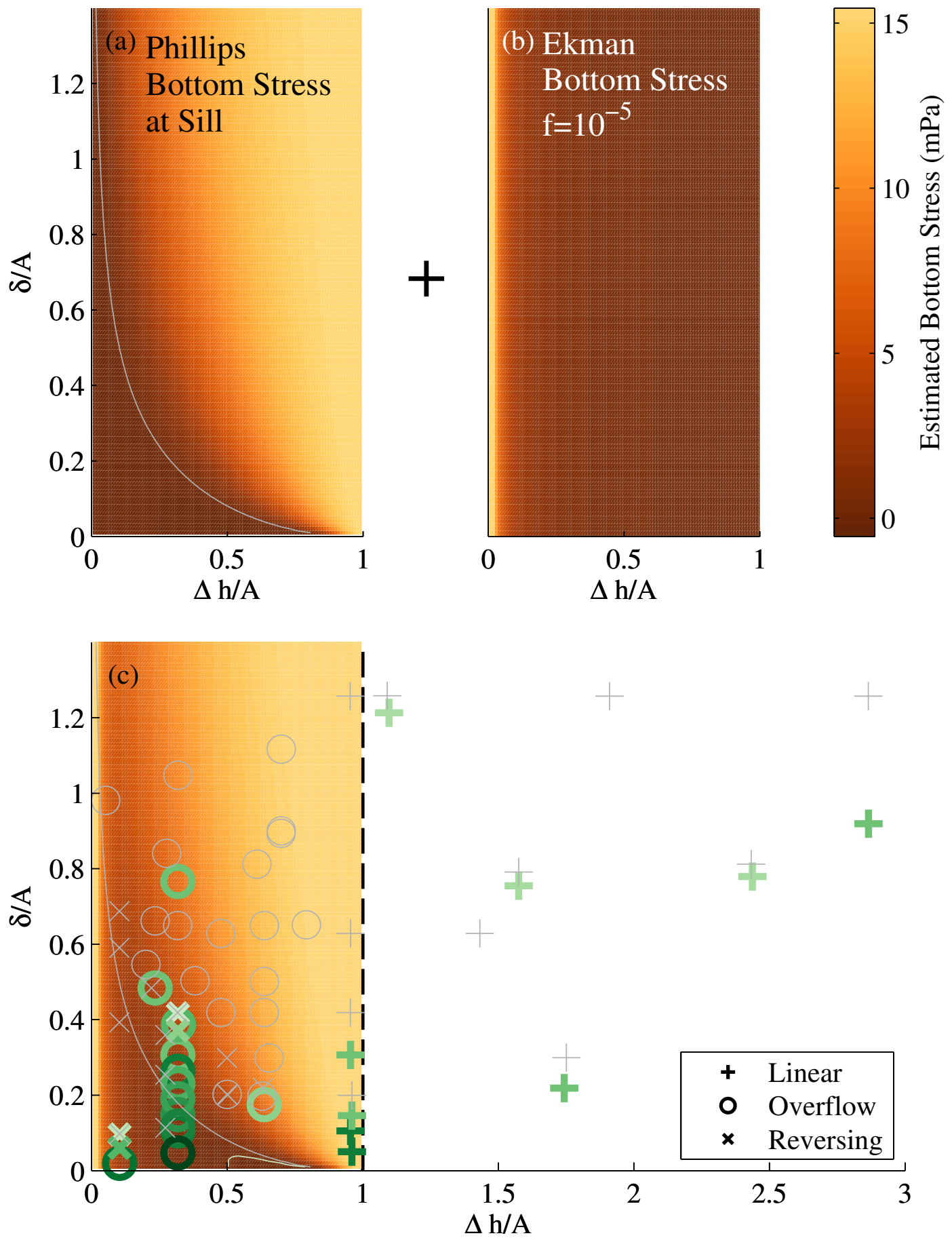
calculating bottom stress than it does when doing a full numerical simulation. Our estimate of the bottom stress (3.25) makes two approximations. The first is the same approximation that we made in the non-rotating analysis: that the bottom stress is near a superposition of the buoyant effect from the maximum slope of each side of the sill. There is no reason to believe that this is less accurate with rotation. The second is that the rotational effect can be superposed on the buoyancy effects. This second assumption was required because the rotational effect has opposite sign on either side of the sill. However, it can be wrong by up to $40 \%$ even over a constant slope.

We can directly calculate the difference between the bottom stress in a Thorpe layer from the sum of the diffusive and rotational parts. The bottom stress associated with the non-rotating diffusive Phillips layer is:

$$
\tau_{P}=\left.\rho_{0} \nu \frac{\partial v}{\partial \eta}\right|_{\eta=0}=\frac{2 \rho_{0} \nu \kappa \cot \alpha}{\delta_{N R}^{2}}=\rho_{0} N \cos \alpha \sqrt{\nu \kappa}
$$

The bottom stress associated with the non-diffusive Ekman layer is:

$$
\tau_{E}=\rho_{0} \nu \frac{f \cos \alpha \cot \alpha}{\mathbb{P}_{r}}
$$

The relationship between them is easily expressed in terms of the slope Burger number

Figure 3-9 (facing page): Even very weak rotation $\left(f=10^{-5}\right)$ dramatically changes the expected parameter range for observing overflows and reversed boundary layers. The upper panel shows the standard regime diagram for $\Delta h / A<1$, with the rainbow colors showing the predicted bottom stress at the sill. The left panel is the predicted bottom stress from the Phillips layer, $\left(\tau_{P 1}-\tau_{P 2}\right)$; the upper right panel is the predicted bottom stress from the Ekman layer, $\tau_{E}$. The dark blue areas are where we expect to see upslope boundary layer fluxes on both sides of the sill ( $\times$ in the lower panel); other colors predict an overflow ( 0 in the lower panel). The lower panel superimposes the two effects, as in equation (3.25), to predict when we expect to see overflows in the full numerical simulations, shown as symbols. Even for very weak rotation, the Ekman effect dramatically changes the prediction, even though it makes a negligible difference to the full numerics (Figure 3-5). 
defined in equation (4.3): $\tau_{P} / \tau_{E}=S$. Their combined bottom stress is:

$$
\tau_{P}+\tau_{E}=\rho_{0} \nu \frac{\cos \alpha}{\mathbb{P}_{r}}\left(N \sqrt{\mathbb{P}_{r}}+f \cot \alpha\right)=\tau_{E}\left(1+S \sqrt{\mathbb{P}_{r}}\right)
$$

The bottom stress associated with the hybrid Phillips/Ekman layer given in equation (3.12) is:

$$
\tau_{R}=\frac{2 \kappa \cot \alpha}{\delta_{R}^{2}}=2 \kappa \cot \alpha\left(\frac{f^{2} \cos ^{2} \alpha}{4 \nu^{2}}+\frac{N^{2} \sin ^{2} \alpha}{4 \nu \kappa}\right)^{1 / 2}=\tau_{E}\left(1+\mathbb{P}_{r} S^{2}\right)^{1 / 2}
$$

where the Prandtl number, $\mathbb{P}_{r}$, is 1 in all of the numerical experiments presented in this chapter. We can see immediately that the two bottom stresses, $\tau_{R}$ and its approximation $\tau_{P}+\tau_{E}$, share some features. If we take the strong rotation limit as $f \rightarrow \infty$, we find $S \rightarrow 0$, and so both $\tau_{R}, \tau_{P}+\tau_{E} \rightarrow \tau_{E}$. The hybrid layer acts like an Ekman layer. If we take the weak rotation limit, where $S \rightarrow \infty$, we find that $\tau_{R} \rightarrow \tau_{E} S \sqrt{\mathbb{P}_{r}}=\tau_{P}$ and $\tau_{P}+\tau_{E} \rightarrow$ $\tau_{E} S=\tau_{P}$. So for both the strong and weak rotation limits, this approximation has the correct asymptotic behavior. However, when $S \sim 1$, the mismatch can be substantial (Figure 3-10).

The approximations of the buoyant and rotational contributions to the bottom stress may compound and make it impossible to get an accurate prediction of where the bottom stress goes to zero and the bottom boundary layer separates from the bottom. Therefore, this technique for predicting the flow geometry may be less applicable for rotating boundary layers than for non-rotating boundary layers.

\subsection{Conclusions}

By examining the complementary and opposing effects of rotation and buoyancy, we have been able to make sense of the sometimes counter-intuitive behavior of boundary layers over rotating two-dimensional topography. We can think of the boundary layer as a hybrid between the buoyancy-driven Phillips layer of the last chapter and an externally 


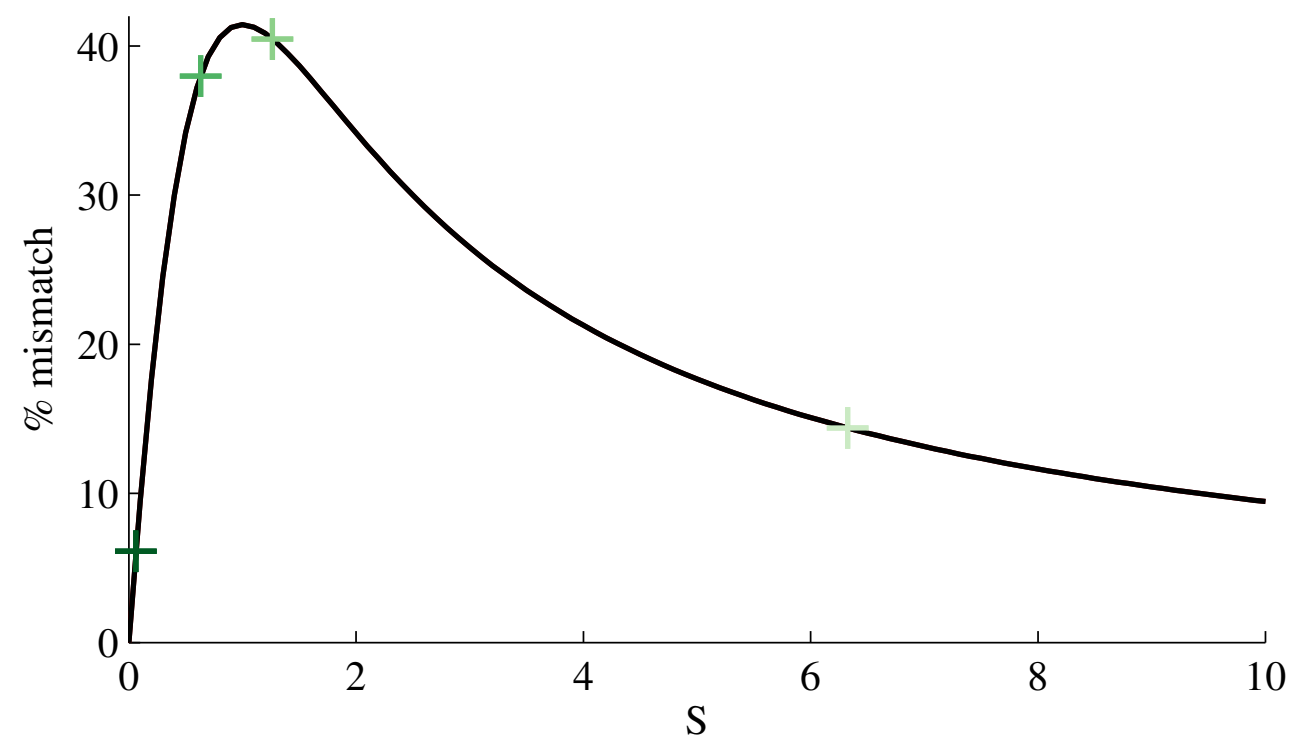

Figure 3-10: The bottom stress of a hybrid Phillips/Ekman layer can be approximated by treating the two layers independently. For both very strong and very weak rotation, this approximation is accurate. However, for intermediate values of $f$, the approximation can be off by as much as $40 \%$. The + show the $S$ values of the series of runs in Figure 3-5. For the weakest rotation case $f=10^{-5}$, the approximation should be accurate to within $15 \%$.

forced Ekman layer, with their relative strengths expressed by the slope Burger number $S$. This approach provides insight into the boundary layer dynamics even though they are ultimately forced only by buoyant effects at the bottom boundary.

The most interesting dynamics arose because buoyant effects respond to the local slope, while rotational effects respond to the overall slope. Therefore, the two can come into conflict where the slope changes, especially where the slope reverses $(\alpha<0)$. The effects can be dramatic: it can change the overall geometry of the flow or reverse the horizontal density gradient and with it the along-isobath velocity.

One thing that rotation cannot change is the total upslope flux in the boundary layer. At steady state, the flux is set by a thermal condition: it must compensate for the diffusive flux divergence at the boundary from diffusion in the far field. However, as Garrett [1990] points out, the direct boundary layer flux may not be the most relevant quantity for understanding the impact of the boundary layer on an ocean basin. The bottom boundary 
layer is always a region of reduced stratification, so the key question is how not how much additional mixing is there in the boundary layer. Rather, it is: How effectively can you exchange the low-stratification water in the boundary layer with the stratified far field? That exchange both impresses the boundary processes on the basin and perpetuates the boundary layer dynamics by maintaining the boundary layer stratification. And even though the flux in the boundary layer is independent of rotation, the exchange between the boundary layer and the far-field is not. Rotation acts directly to reduce the exchange between the boundary layer and the far field by making the boundary layer more Ekman-like, and so less sensitive to local changes in the topography that can force exchanges. In the limit of a rotationally-dominated boundary layer, there is essentially no exchange, meaning the topography is essentially negligible for the upslope and vertical velocity fields. Dynamically, we return to the constant slope Thorpe solution presented in the introductory section of this chapter. This happens even though the whole system is driven by the buoyant effects that are sensitive to the local topography.

These two-dimensional rotating boundary layers are potentially less directly applicable to understanding canyons than the non-rotating boundary layers in the last chapter. They all depend on unlimited along-isobath flows, which can be substantial. For the numerical experiments shown in Figure 3-5, $u_{\infty}$ ranges from $1-15 \mathrm{~cm} / \mathrm{s} ; u_{\infty}$ is almost $5 \mathrm{~cm} / \mathrm{s}$ for the realistic value $f=10^{-4}$, substantially more than the upslope velocity. It would be extremely difficult to maintain such a velocity inside a canyon only 10-20 km wide, and there is no indication of it in the two-year-long current meter record taken in the $22^{\circ} \mathrm{S}$ canyon in the BBTRE. [Thurnherr et al., 2005]. However, even though these boundary layers may be less realistic than the non-rotating version seen in the previous chapter, the lessons that we learned here will be essential in understanding the three-dimensional dynamics presented in the next chapter. 


\section{Chapter 4}

\section{Diffusion-Driven Boundary Layers in}

\section{Canyons}

This thesis has always had two primary goals: First, to better understand the basic physics of how stratified fluids interact with varying boundaries. Second, to apply that knowledge to better understand how ocean-bottom topography affects the mixing, stratification, and transport of the ocean, especially in the context of abyssal canyons. Toward these ends, we started with the simplest possible boundary-layer/topography interactions, and carefully added complexity while striving to maintain a detailed understanding of the physics. We went from a non-rotating boundary layer over a bottom with a single change in slope, to isolated two-dimensional sills, then periodic sloping topography, before introducing rotation. This process showed us previously unknown mechanisms through which abyssal ocean mixing can be increased through the spontaneous development of small-scale boundary layer overflows and then how that mixing can be communicated to the far field through exchange flows between the boundary layer region and the far field.

In this chapter, we arrive at the first fully three-dimensional dynamics. In so doing, we study something that actually looks like a canyon, though it remains highly idealized. Introducing sidewalls into a domain that was previously infinite creates a new dynamical regime: a geostrophic boundary layer outside the viscous bottom boundary layer. Water is constantly exchanged between the viscous layer and the geostrophic layer, which extends 
hundreds of meters from the bottom. As water parcels move between the two, they follow helix-shaped trajectories that fill the canyon bottom. This has profound implications for the large-scale patterns of currents and density that we expect to see in ocean canyons. It also changes the rate of net transport we expect to see in abyssal canyons in a way that may help reconcile tracer and current meter observations in the one canyon where we have both. When we introduce topography into our idealized canyons, we find that once again it can both increase mixing and communicate that mixing by forcing exchanges with the far field. The combination of three-dimensional, canyon-filling viscous and geostrophic layers and even very simple topography creates enormously complicated fluid trajectories. This study reveals the new opportunities for mixing and exchange-for engaging the topography in the life of the fluid - that the third dimension provides.

\subsection{Introduction}

In this chapter, I present a series of idealized numerical simulations designed to illuminate the dynamics of viscous, diffusive boundary layers in canyons. These boundary layers have been well-described for uniform unobstructed slopes by Phillips [1970] and Thorpe [1987]. As we saw in the previous chapters, the no-flux bottom boundary condition leads to bending isopycnals near a sloping bottom, and the resulting pressure gradient drives a bottom intensified upslope flow. If the diffusivity $\kappa$, the viscosity $\nu$, the stratification $N$, and background slope $\alpha_{0}$ are constant, the upslope velocity is:

$$
v(\eta)=2 \kappa \gamma_{R} \cot \alpha e^{-\gamma_{R} \eta} \sin \left(\gamma_{R} \eta\right)
$$

where $\eta$ is the slope-normal coordinate as shown in Figure 4-1 and $\gamma_{R}$ in a reciprocal boundary layer thickness that depends on both the rotation and buoyancy of the flow:

$$
\gamma_{R}=\left(\frac{f^{2} \cos ^{2} \alpha_{0}}{4 \nu^{2}}+\frac{N^{2} \sin ^{2} \alpha_{0}}{4 \kappa \nu}\right)^{1 / 4}=\gamma_{E}\left(1+S^{2} \mathbb{P}_{r}\right)^{1 / 4} \sqrt{\cos \alpha_{0}}
$$


$\gamma_{E}$ is the reciprocal Ekman layer thickness $\sqrt{f / 2 \nu}$, and $\mathbb{P}_{r}$ is the Prandtl number, $\nu / \kappa$. In the last chapter, we found that $S$, the slope Burger number, was the controlling parameter for many of the dynamics in rotating, diffusive boundary layers. It is:

$$
S=\frac{N \sin \alpha}{f \cos \alpha} \sim \frac{\text { Buoyancy }}{\text { Rotation }}
$$

One of the most important features of this boundary layer is that its total volume flux is dependent only on the bottom angle and the diffusivity:

$$
Q_{0}=\int_{0}^{\infty} v \mathrm{~d} \eta=\kappa_{\infty} \cot \alpha_{0}
$$

If the bottom angle changes, the volume of water flowing through the boundary layer also changes. This holds regardless of the value of the Coriolis parameter $f$.

We find that many of these features carry over quite directly into the canyon context. There is a narrow-channel limit, where the rotating boundary layer is so constrained that it cannot feel the effects of rotation and so returns to the two-dimensional non-rotating boundary layer, but it is not very relevant to the ocean. In order to reach it, the canyon has to be narrow compared to the thickness of the sidewall viscous boundary layers, which have thickness on the order of the Ekman scale $\sqrt{2 \nu / f} \sim \mathrm{O}(10 \mathrm{~m})$ wide. In an ocean canyon, around $20 \mathrm{~km}$ wide, the volume flux, upslope velocity field, and boundary layer thickness from the uniform slope are all roughly or precisely applicable.

However, at least one feature of the uniform slope solution cannot carry over. Thorpe found that the along-isobath velocity far from the bottom boundary was not a free parameter or an arbitrary external forcing, but a necessary part of the steady-state boundary layer solution. Obviously, in the presence of canyon walls, the along-isobath velocity far from the bottom must go to zero. For this reason, there is no true wide-channel limit; no canyon can be wide enough to produce the along-isobath flow profile from the uniform slope solution.

This study is focused on understanding flows in canyons that are representative of ocean 
canyons, which turn out to be neither very wide nor very narrow, from a dynamical perspective. They have widths of 5-25 km and bottom slopes on the order of $10^{-3}-10^{-2}$ in the along-canyon direction. Since this is the first study of such canyons, we concentrate on the steady-in-time dynamics. We first seek to understand a canyon that has a uniform bottom slope in the along-canyon $(\xi)$ direction, then introduce isolated sills and study their effects. Bottom topography that varies everywhere is left for future study.

After detailing the numerical methods in the following section, I will demonstrate that canyons are subject to the same transport constraint that Phillips and Thorpe found for boundary layers over uniform slopes in non-rotating and rotating domains, given in equation (4.4). In section 4.4 , I devote the largest piece of this chapter to developing a thorough understanding of the dynamics of a canyon with straight vertical sidewalls and a constant slope bottom, including both Eulerian and Lagrangian descriptions of a new dynamical regime - the geostrophic boundary layer. It has interesting implications for along canyon transport, and this section provides an opportunity to compare the theory to observations, at least in broad strokes. In the final section, I introduce isolated sills into the canyons, and we find that topography once again can both increase mixing and communicate that mixing from the boundary region to the far field.

\subsection{Numerical Methods}

The numerical simulations presented in this chapter use a similar methodology to those presented in the previous chapters presenting two-dimensional boundary layer studies. Once again, I use the Regional Ocean Modeling System (ROMS) to study bottom boundary layers in an idealized domain. This is hydrostatic, primitive equation model with terrainfollowing coordinates [Shchepetkin and McWilliams, 2005]. These coordinates allow especially smooth and detailed resolution of the bottom boundary layer on varying topography, the particular object of this study. To ensure that the relevant dynamics were fully resolved, the vertical resolution in the bottom boundary layer was as high as $0.5 \mathrm{~m}$. Far from the 
boundary, the vertical resolution fell to $15 \mathrm{~m}$ or sparser.

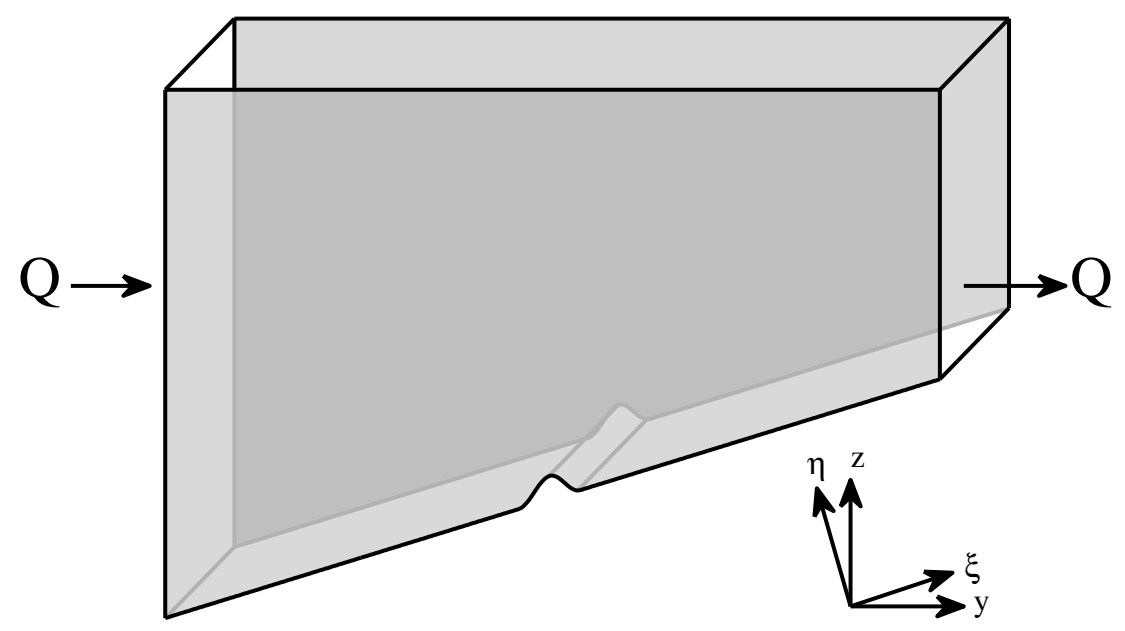

Figure 4-1: The computational domain is a channel with two walls (parallel to the page) and two open lateral boundaries. The channel has a sloping bottom, and in some simulations has varying topography superimposed on the slope. The total volume flux $Q$ through the domain is held constant at the value derived in equation (4.9). When I describe the domain, it is always from the perspective of a viewer looking up the slope, in the same direction as the $Q$ arrows in this figure.

I converted the two-dimensional domain of the previous chapters to a canyon by expanding the domain in the along-isobath direction and adding vertical side wall boundaries (Figure 4-1). The mid-ocean ridge flank canyons that inspired this study have a characteristic width of $20 \mathrm{~km}$ and a characteristic length of around $1000 \mathrm{~km}$. Of course, it would not be feasible to resolve a boundary layer that is tens of meters thick in a domain of that size. Therefore, domain size was chosen to have a realistic size in the cross-canyon direction and to be long enough to have a substantial section with uniform dynamics in the along-canyon direction. In addition, I required that the domain be deep enough to ensure the bottom boundary layers did not feel the presence of the free surface. I found that these requirements were met when the domain was at least three times longer than it was wide, and when the shallowest part of the domain was at least 35 times the bottom boundary layer thickness. Many simulations were successfully duplicated in domains $2-3 \mathrm{~km}$ deeper and in domains as much as ten times longer than they are wide, in order to be certain that the 
results were not sensitive to the domain size. All of the simulations presented in this chapter have domains with flat bottoms, but I duplicated several of the simulations in domains with rounded and other-shaped bottoms, and found no overall difference in the canyon circulation.

Unlike the two-dimensional studies, in a canyon there is no known analytical solution that can validate the numerics. Therefore a key numerical test was whether the solution over a constant-slope bottom was truly uniform in the along-canyon direction. I found excellent along-canyon uniformity in all the simulations; Figure 4-2 shows an example for both the along-canyon and cross-canyon velocities in a canyon that is $10 \mathrm{~km}$ wide and $30 \mathrm{~km}$ long.

The domain has two types of lateral boundary conditions. The canyon walls are closed lateral boundary conditions that enforce the no-normal-flow condition at each side of the canyon. For the velocity tangent to the wall, I used a no-slip sidewall boundary condition. However, I duplicated several of the simulations with with free-slip sidewall boundaries, and found that this had no discernible effect on the dynamics in the canyon. This is not surprising, as the numerical simulations cannot resolve the sidewall viscous boundary layer that would enforce the no-slip condition. The thickness of the sidewall viscous layer is given by the Ekman scale $\sqrt{2 \nu / f} \sim \mathrm{O}(10 \mathrm{~m})$, which for these parameter values is larger than the thickness of the Stewartson [1957] $E^{1 / 3}$ and $E^{1 / 4}$ layers. We will see in the subsequent sections that the thickness scale of the sidewall currents is more than a kilometer. Given this scale separation and the insensitivity of the results to the sidewall boundary condition, the horizontal resolution used, 150-250 m, is adequate.

In addition, I used open boundary conditions at either end of the canyon, as in the two-dimensional studies. The net bottom slope in the domain made a periodic conditiontypical in channel studies-impossible. I specified the barotropic volume flux through the domain at both ends of the canyon. We can calculate this flux a priori, as shown in the next section. Since there can be no net along-isobath (cross-canyon) flux in the domain, there was no net free-surface slope. I used standard ROMS radiation conditions for the 

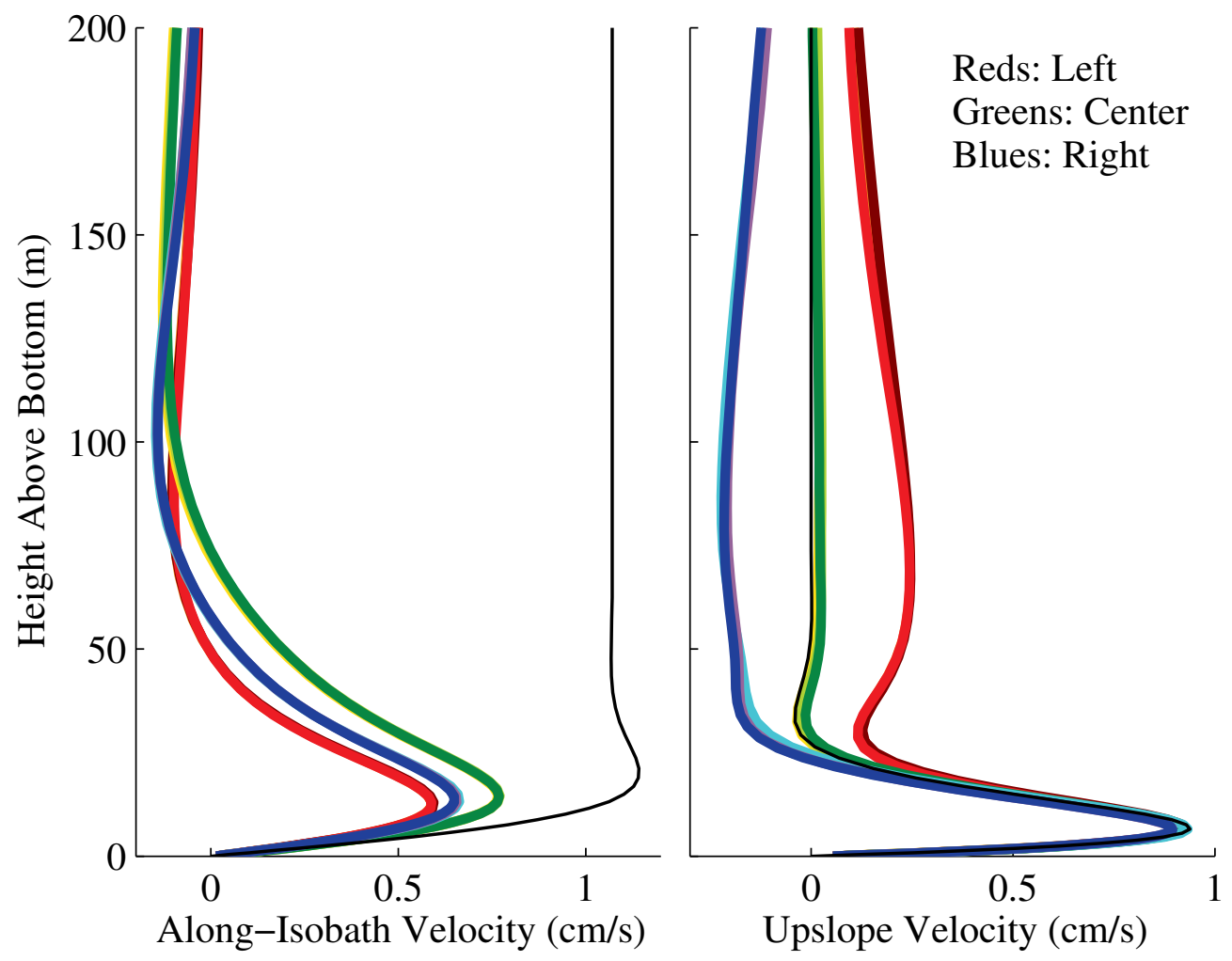

Figure 4-2: One of the main computational challenges was ensuring the solution was truly uniform in the along-canyon direction. Here we see nine profiles of along-isobath (left) and upslope (right) velocity, taken at three different locations in the along-canyon direction and three in the cross-canyon direction. The thin black lines are the analytical velocity profile for an infinite constant slope with the same values of $f, \nu, \kappa, N$, and $\alpha_{0}$. The profiles at different along-canyon positions line up so closely that it is difficult to see that there is more than one profile being plotted. This gives us confidence that we are simulating the infinite-channel case. 
inflowing baroclinic velocity and for the temperature and salinity. Since the temperature and salinity use radiation conditions, the stratification can erode with time, but this happens so slowly that the initial stratification was not significantly eroded or altered by the end of the simulations.

The simulations began with a constant stratification and zero velocity everywhere in the domain, and were run until the velocity field had come to an approximately steady state. The boundary layers that develop are unforced by any stress or far-field velocity, but arise spontaneously through the interaction of stratified fluid and sloping boundaries. No forcing of any kind was applied except for the specified barotropic flux through the domain. In the simulations, that flux is carried almost entirely in the bottom boundary layer, and the velocities all decay to zero far from the boundary, so we can be confident that the barotropic flux boundary condition is not inadvertently introducing a forcing, but merely matches the dynamics that arise unforced. Because the viscous bottom boundary layer drives a geostrophic circulation, the time to steady state is set by both the diffusive timescale of the the bottom layer and by the inertial timescale. The diffusive timescale is typically a few hours in the parameter ranges we study, and the inertial timescale is about a day. This means that these simulations take many times longer to equilibrate than the twodimensional simulations that depended only on the diffusive timescale. The simulations presented in this chapter were allowed to equilibrate for a minimum of three days, and often as much as 10 days. After this time, the acceleration term in the momentum balance was less than $1 \%$ the size of the terms involved in the steady-state momentum balance.

There was no evidence of Rossby waves in the time adjustment of the canyon circulation. The dispersion relation for topographic Rossby waves is:

$$
\omega=\frac{\alpha_{0} g}{f} \frac{k}{1+\frac{g H_{0}}{f^{2}}\left(k^{2}+l^{2}\right)}
$$

where $k$ is the wavenumber in the along-isobath direction, $l$ is the wavenumber in the upslope direction, and $\alpha_{0}$ is the background slope [Cushman-Roisin, 2007]. By this dispersion 
relation, larger waves propagate faster. For the parameter values used in the numerical simulations presented here, the largest wave that will fit in the canyon $(k=2 \pi / L, l \rightarrow 0$, where $L$ is the canyon width) has a phase speed of $2 \times 10^{-3} \mathrm{~m} \mathrm{~s}^{-1}$. It would take about two months for it to propagate across the canyon, much longer than the simulations require to reach an approximate steady-state. Therefore, it is not surprising that there is no sign of 'westward' phase propagation in a Hovmöller diagram of along-canyon velocity. In addition, Kelvin waves are not significant to the dynamics because the flow field is uniform in the along-canyon direction.

I use a prescribed eddy viscosity and diffusivity, $\nu_{e d d y}$ and $\kappa_{e d d y}$ of $5 \times 10^{-3} \mathrm{~m}^{2} \mathrm{~s}^{-1}$ to approximate turbulent mixing processes. This value is taken from canyon microstructure observations presented in Thurnherr et al. [2005].

Lagrangian trajectories were a key tool for exploring the numerical simulations. The trajectories were calculated off-line, with the Eulerian velocity fields generated by ROMS. The ROMS simulations - which lasted days - were too short to track a water parcel's motion on the scale of the entire canyon-which takes years-so we repeated the final velocity field from the ROMS simulations many times over. This artificially-perfectly-stationary velocity field allowed me to calculate trajectories up to ten years long in some cases, more than 1000 times longer than it was feasible to run ROMS. To ensure that the trajectories were not an artifact of this artificially stationary velocity field, I also calculated trajectories with a random velocity added to the Lagrangian velocity at each timestep to parameterize the effects of sub-grid-scale turbulence. Even turbulence 200 times stronger than the eddy diffusivity used in ROMS had no significant effect on the trajectories. For example, Figure 4-3 shows three trajectories with random 'turbulent' velocities corresponding to $\kappa=0$, $5 \times 10^{-3}$, and $1 \mathrm{~m}^{2} \mathrm{~s}^{-1}$, and they all look almost identical. The trajectories appear to be robust to small temporal variations in the flow field.

I calculated the trajectories with TRACMASS, a software package developed by Döös [1995]. TRACMASS solves the trajectory path through each grid cell by directly solving a differential equation for the water parcel's motion based on the velocities on the faces of 


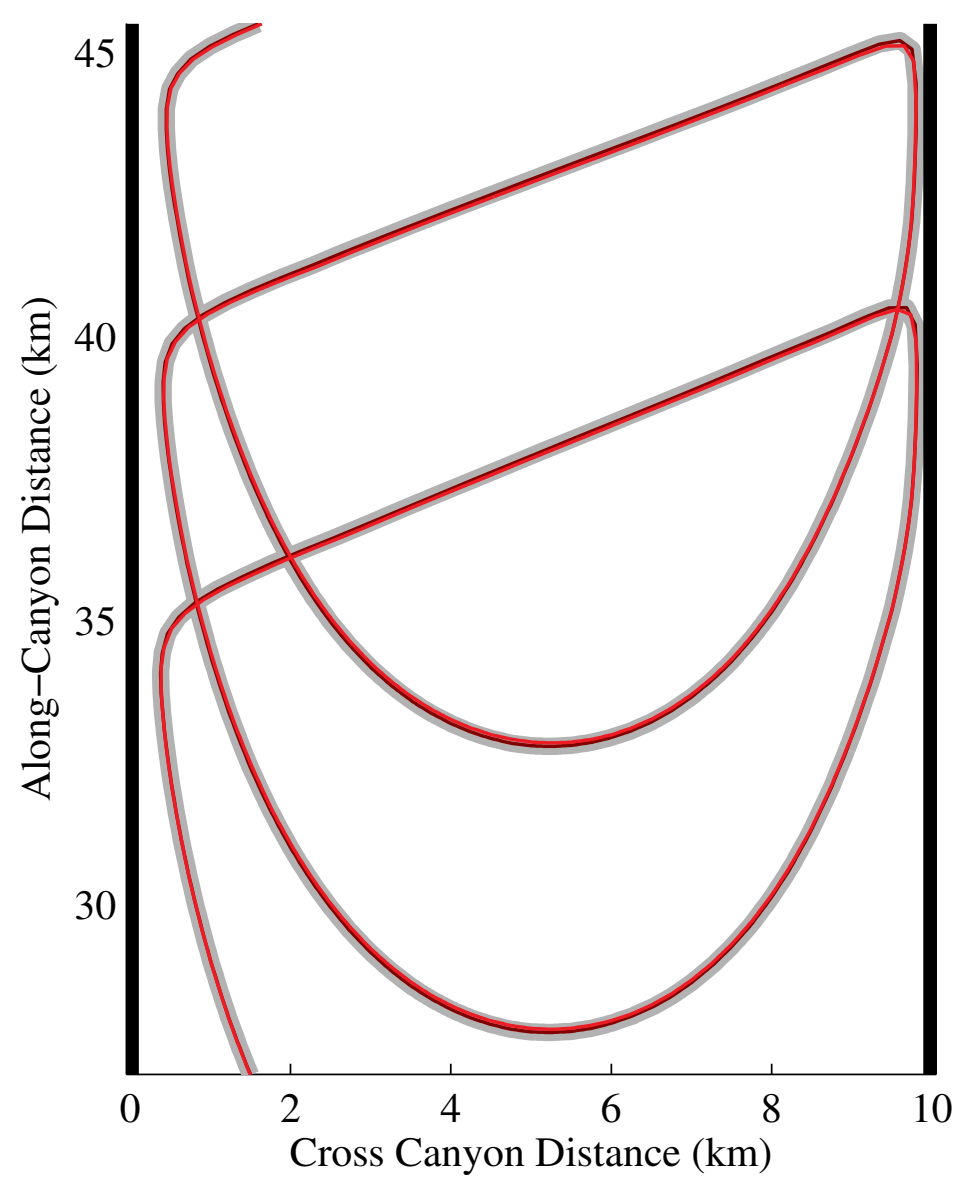

Figure 4-3: Adding additional turbulence into the trajectory calculation has no significant effect on the trajectories. In the above plan-view of a $10 \mathrm{~km}$ wide canyon, the thick grey line is a trajectory calculated with no additional time-dependence or sub-grid-scale turbulence. The dark red line shows the same trajectory calculated with a random turbulent velocity corresponding to the diffusivity used in the original numerical experiment, $\kappa=5 \times 10^{-3} \mathrm{~m}^{2} \mathrm{~s}^{-1}$, added at each timestep of the trajectory calculation. The bright red line shows the same calculation with a hugely increased eddy diffusivity of $\kappa=1 \mathrm{~m}^{2} \mathrm{~s}^{-1}$. All three methods produce nearly identical trajectories.

the grid box. Therefore, the trajectories have much higher spatial precision than the grid resolution. I seeded each canyon simulation with a cross-canyon sheet of more than 500 trajectories that spanned the thickness of the viscous boundary layer and the central $50 \%$ of the canyon width. I truncated trajectories that entered regions where the velocity field might not be fully reliable: the viscous boundary layer on the sidewall, which we could 
not properly resolve; near the open lateral boundaries; or near the free-surface. Since the trajectories are calculated at significantly higher resolution than the original velocity field, this truncation did not require discarding all of the trajectories that entered the grid cell closest to the canyon sidewalls.

\subsection{Volume Flux Through the Canyon}

One of the most important results from Phillips [1970] original paper on viscous, diffusive boundary layers is that the total volume flux in the boundary layer can be calculated a priori, by integrating the density equation, even if the velocity field is unknown. It took the elegant form:

$$
Q=\kappa \cot \alpha_{0}
$$

$\kappa$ is the diffusivity and $\alpha_{0}$ the constant slope of Phillips's infinite, smooth boundary.

The transport can be constrained because the total transport in the the boundary layer must be such to perfectly balance the flux divergence at the bottom boundary in a diffusive, stratified fluid. There is a constant diffusive density flux up in the interior, but no flux at the insulating boundary, so the interior flux must be fed by advecting dense fluid from deeper in the water column.

Thorpe [1987] found the same flux constraint after introducing rotation to the infinite constant slope solution. This is unsurprising because it is essentially a thermal condition, so rotation should be immaterial. Similarly, we might expect the same constraint to hold in a canyon: provided no density fluxes arise from the sidewalls, the same bottom flux divergence must be compensated by the same bottom flux. This is true even though the general features of Thorpe's solution for a two-dimensional rotating boundary layer are impossible in a canyon. He found that an along-isobath flow infinitely far from the boundary was needed to keep the rotational and diffusive effects in balance at steady state, and such an along-isobath flow would be blocked by the canyon.

However, the flux divergence is unaffected by the addition of canyon walls to the sys- 
tem, so we should expect the condition to hold. We can easily demonstrate this condition for a theoretical long canyon with straight vertical walls at $x=0, L$ and a bottom slope $\alpha_{0}$. Begin with the density equation:

$$
\frac{\partial}{\partial x}(u \rho)+\frac{\partial}{\partial \xi}(v \rho)+\frac{\partial}{\partial \eta}(w \rho)=\kappa \frac{\partial^{2} \rho}{\partial x^{2}}+\kappa \frac{\partial^{2} \rho}{\partial \xi^{2}}+\kappa \frac{\partial^{2} \rho}{\partial \eta^{2}}
$$

Here, the coordinates $(x, \xi, \eta)$ are the across-canyon (along-isobath), along-canyon (upslope), and slope-normal directions, and have associated velocities $(u, v, w)$, as shown in Figure 4-1. We assume a constant background stratification $N$, so $\frac{\partial^{2} \rho}{\partial \xi^{2}}=0$. If we integrate in $x$ across the width of the canyon, we can eliminate the first term on the left because $u$ must go to zero at the sidewalls. Similarly, since we assume there are no fluxes of heat or salt through the sidewalls, the first term on the right must also integrate to zero. Following Phillips and Thorpe, we assume that the velocity field is uniform in the alongslope $(\xi)$ direction, and that the density field only varies in $\xi$ with the constant background stratification. This means $\frac{\partial \rho}{\partial \xi}$ is known and constant. After integration, we are left with:

$$
L \frac{\partial \rho}{\partial \xi} \bar{v}+\int_{0}^{L} \frac{\partial}{\partial \eta}(w \rho) \mathrm{d} x=\kappa \int_{0}^{L} \frac{\partial^{2} \rho}{\partial \eta^{2}} \mathrm{~d} x
$$

where $\bar{v}$ is the along-canyon velocity as a function of distance from the bottom, averaged in the across-canyon direction. After a second integration in the slope-normal direction from $\eta=0$ to $\eta \rightarrow \infty$, we can cancel the $w$ term, because $w$ must be zero at the bottom and infinitely far from the boundary. This leaves us with an expression for the depth-integrated volume flux $Q$ :

$$
Q=\int_{0}^{\infty} \bar{v} \mathrm{~d} \eta=\left(\left.\kappa \int_{0}^{L} \frac{\partial \rho}{\partial \eta}\right|_{0} ^{\infty} \mathrm{d} x\right) /\left(\frac{-L \rho_{0} N^{2} \sin \alpha_{0}}{g}\right)=\kappa \cot \alpha_{0}
$$

We know $\frac{\partial \rho}{\partial \eta}$ at the bottom boundary $(\eta=0)$ from the no-flux bottom boundary condition, and infinitely far from the boundary $(\eta \rightarrow \infty)$ it is set by the background stratification $N$. Note that we were able to switch the order of integration in this case because the limits 
of integration were constant. As expected, we find the same total flux through the domain generated by the thermal boundary layer over the sloping bottom as Phillips found in the two-dimensional case. If the sidewalls are not straight, we would expect thermal sidewall boundary layers like the bottom boundary layer, and the distribution of boundary layer flux would depend on the specific geometry of the topography. Woods [1991] discusses the related case of vertical boundary layer fluxes in closed containers with slowly varying sidewall slopes.

Equation (4.9) is a valuable result for two reasons. First, we need it to set the barotropic flux open boundary condition in the numerical simulations. Second, since the boundary layer volume flux depends on the bottom slope in the same way as in the two-dimensional case, we can have much more confidence in applying the reasoning we developed there to understanding the circulation in a canyon.

\subsection{Circulation in a Canyon with a Constant Bottom Slope}

In the two-dimensional systems that we studied in the previous chapters, there were two significant dynamical regimes: the bottom boundary layer, and the inviscid far field. Introducing canyon walls introduces a variety of new complications, including two additions to the list of significant dynamical regimes:

1. the viscous and diffusive bottom boundary layer;

2. a geostrophic layer, above the bottom layer, where water is constantly exchanged with the bottom layer;

3. a corner transition region between the bottom and geostrophic layer; and

4. the far field.

These regimes are illustrated schematically in Figure 4-4. (The cross-canyon sections are plotted such that the viewer is looking up the slope, and positive $\xi$ is into the page. Whenever I refer to 'left' and 'right' in the canyon, it is with this perspective.) 


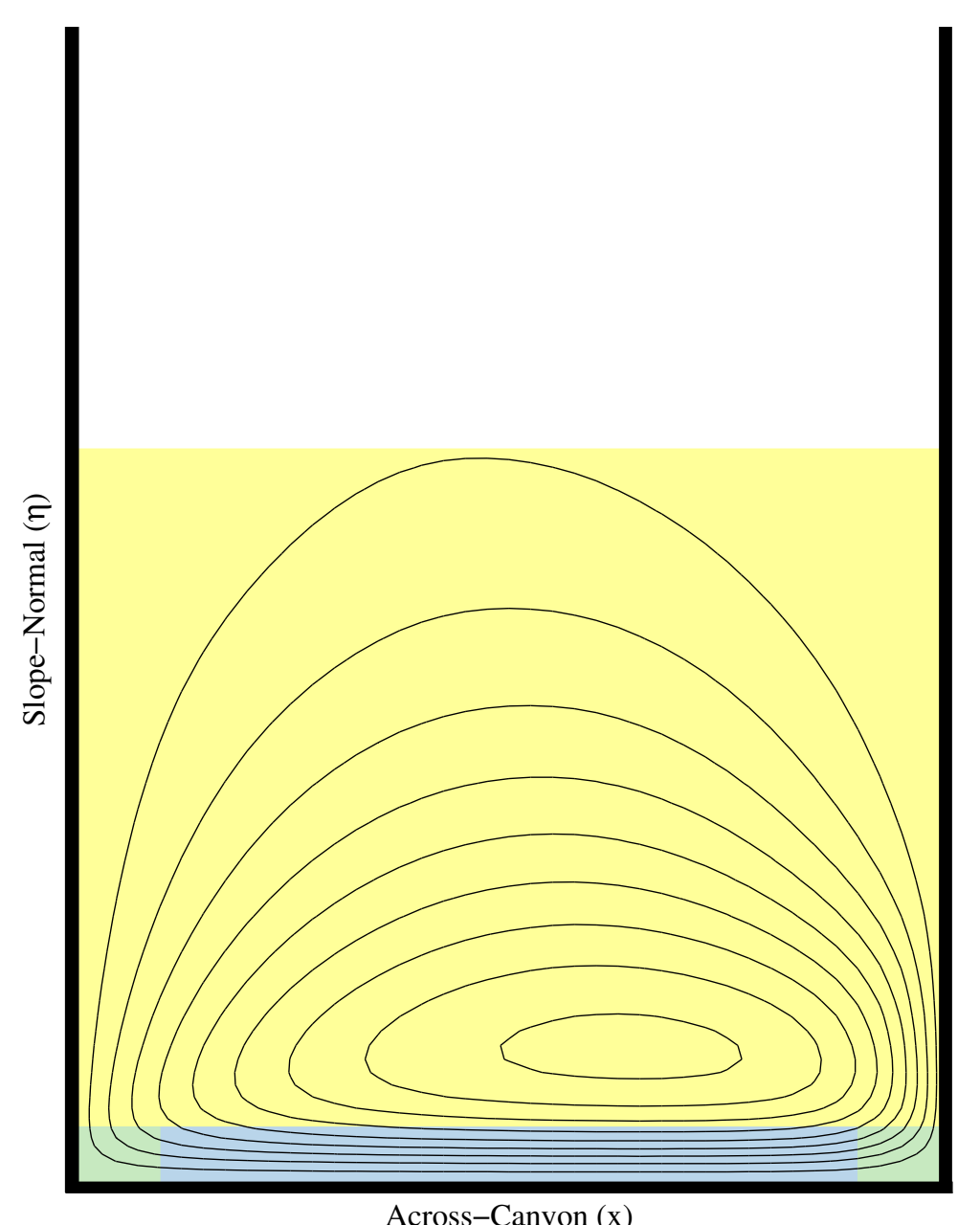

Figure 4-4: In a canyon, there are four distinct dynamical regimes, each with a distinct momentum balance. They are: the viscous and diffusive bottom boundary layer (blue); the geostrophic layer (yellow); the transition region between them (green); and the far field (white). The overlaid black contours show the $x \eta$ streamfunction, with a rapid alongisobath flow in the bottom boundary layer and a slower, more spread out recirculation in the geostrophic layer.

Let's begin by looking at the most familiar of the dynamical regimes, the bottom boundary layer. In the upslope velocity (shown in Figure 4-5b), we see a thin bottom layer, with a current going up the slope. The upslope flow is remarkably uniform in the along-isobath direction. In the slope-normal direction, the velocity varies very similarly to the infiniteslope case; profiles of the canyon and the infinite-slope velocity are compared in Figures 
4-2a and 4-6b. The bottom boundary layer is so similar to the two-dimensional case, in fact, that if we plot the upslope flux $Q$ just in that bottom layer, ignoring all the dynamics above it, we see that it matches the predicted value for the whole canyon from equation (4.9) almost exactly (Figure 4-6a).

This bottom layer also has an along-isobath velocity that arises because the pressuredriven upslope flow is deflected to the right by rotation. The velocity scale of the alongisobath flow is the same as in the infinite slope case, but the overall structure of the velocity is very different because there can be no along-isobath flow at $\eta \rightarrow \infty$ (Figure 4-2a).

Returning to Figure 4-5c, there is a clear signature of the viscous layer in the vertical velocity (in the $z$-direction, aligned with gravity), but no evidence of it in the slope-normal ( $\eta$-direction) velocity (Figure 4-5d). Water parcels change elevation as they move along the sloping bottom, but for most of the canyon width, water is not getting closer or farther from the bottom-it is not entering or leaving the viscous bottom layer.

Above the bottom layer is the most conspicuous feature in the canyon circulation: two prominent along-canyon jets, one going in the upslope direction, and one going down. The down-slope jet is a little sharper and more intense, but the two jets are overall nearly symmetrical. The horizontal scale is $1-2 \mathrm{~km}$, vertical scale $200 \mathrm{~m}$, and the velocities are the same order as the velocity in the viscous layer. The jets begin more or less directly above the viscous layer. Since the total volume flux in the domain is carried mostly in the viscous bottom layer, we know the up-and down-canyon jets on either side of the domain cancel each other out almost perfectly (Figure 4-6) - the upslope current on the left side of the domain carries the same volume flux as the downslope current across from it.

These jets are conspicuously absent from the vertical ( $z$-direction) velocity, but reappear in the slope-normal ( $\eta$-direction) velocity. This means that they represent water-lots of water-entering or leaving the viscous layer, but doing it by moving nearly horizontally. Above the bottom boundary layer, water is moving in in the $x y$ plane, not the $x \xi$ plane, and so it has to move in or out of the bottom boundary layer. 


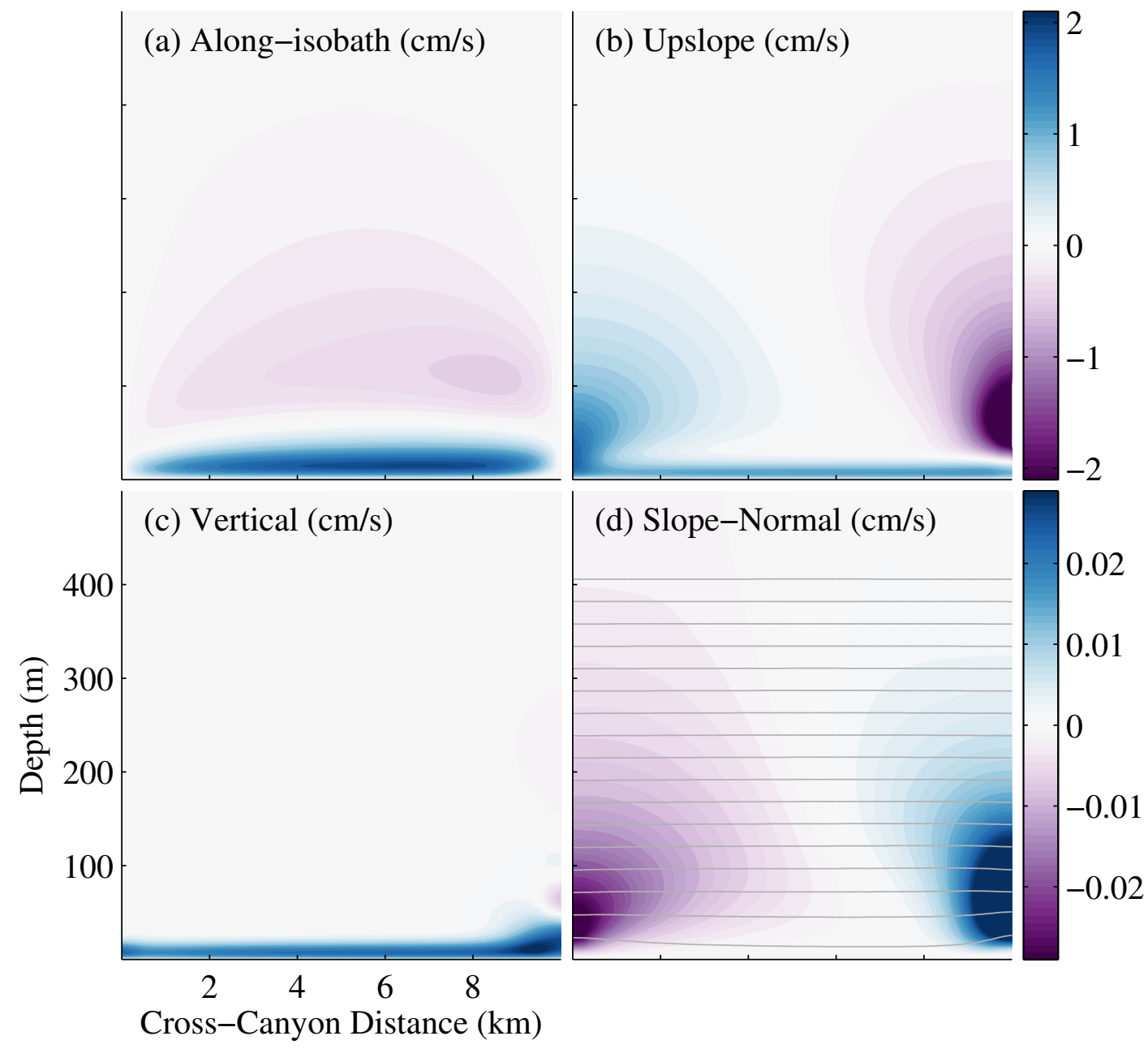

Figure 4-5: A channel with a sloping bottom has an up-slope viscous bottom boundary layer and a complicated set of secondary circulations. Above are the velocity components in a cross-canyon section, plotted such that the reader is looking in the upslope (positive $\xi$ ) direction by looking into the page. First, there is an an along-isobath bottom current with a return flow farther from the bottom (a). Two along-canyon jets form (b), one flowing upslope and one down-slope, both banked on their respective left wall. The vertical (alignedto-gravity) velocity (c) shows only the signature of the up-slope viscous bottom boundary layer, but the slope-normal velocity (d) shows us that the fluid in the jets is entering or leaving the boundary layer. (d) also shows isopycnals, which bend up at both canyon walls near the canyon bottom. The total density range shown is $0.4 \mathrm{~kg} \mathrm{~m}^{-3}$. The isopycnal tilt is in geostrophic balance with the along-canyon flow. 


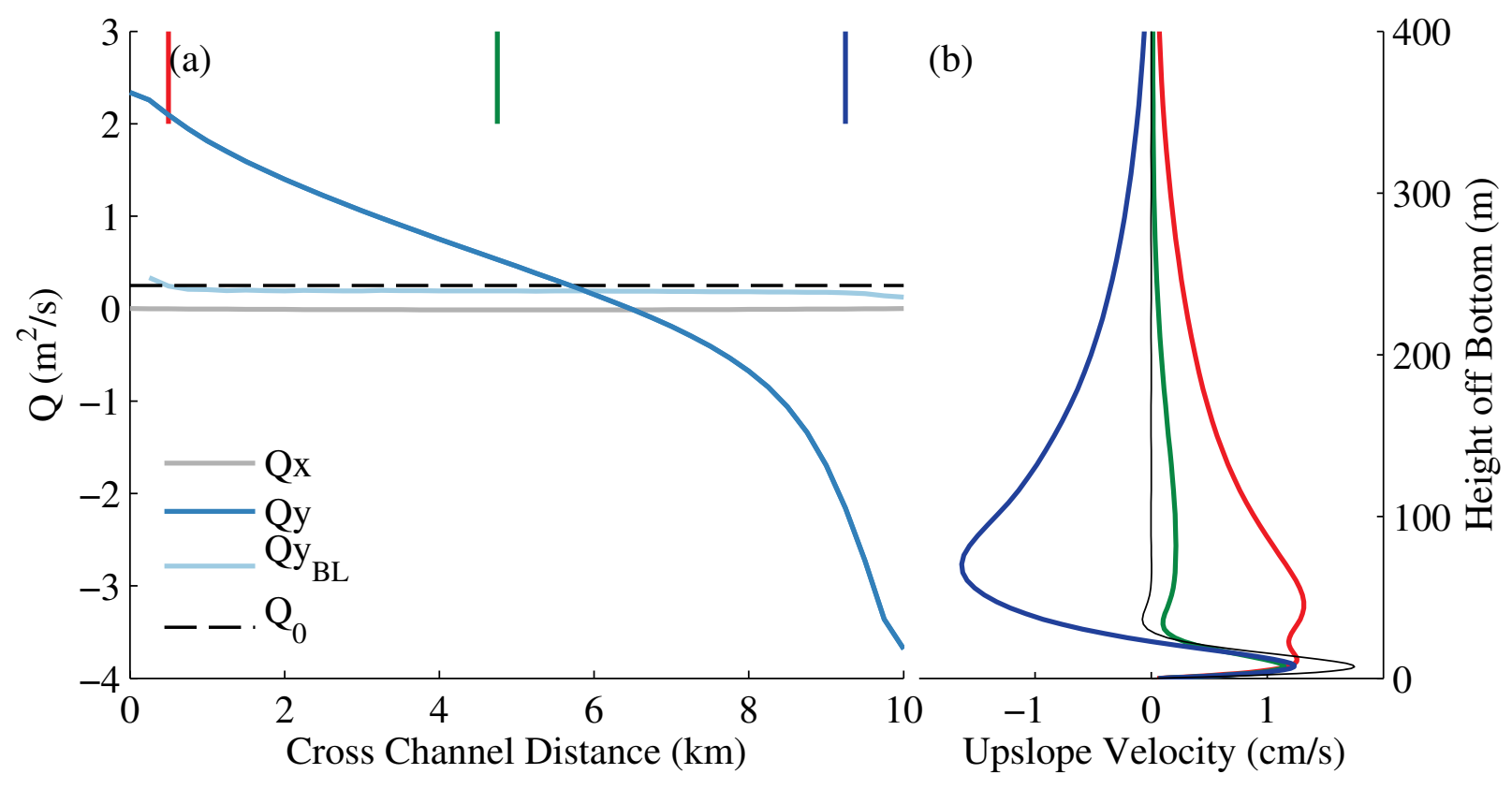

Figure 4-6: The volume flux per unit width in the viscous bottom boundary layer (a, light blue) is almost the same as the total up-slope flux in the canyon (a, dashed black), given in equation (4.9). Therefore, the flux in the upslope and downslope jets (a, dark blue) must cancel almost perfectly. The net across-canyon flux (a, grey) is zero, as expected. The upslope velocity profiles (b), taken at the across-channel locations indicated by the colored tick marks on (a), show a viscous bottom boundary layer that is very similar to the two-dimensional analytical solution (black), with a strong jet-like circulation above it. 


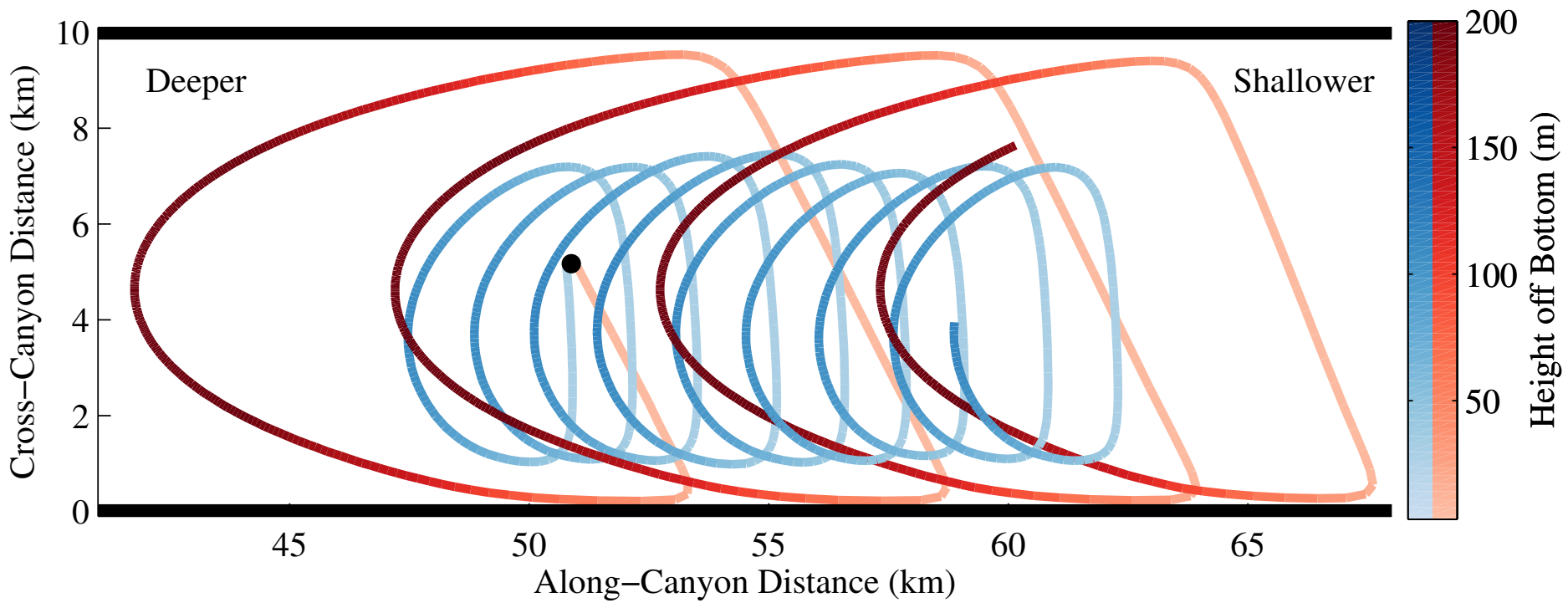

Figure 4-7: Two water parcels follow spiraling trajectories as they move between the viscous and geostrophic boundary layers. Starting at the large black dot, the red trajectory begins near the maximum in upslope velocity in the viscous boundary layer, and moves upslope (to the right in this figure), while being pushed to its right. When it hits the sidewall, it is forced out of the viscous boundary layer and into the geostrophic boundary layer. It moves isopycnally away from the slope and slowly circulates across the canyon, before reentering the viscous boundary layer on the left side of the canyon. The color shows $\eta$, the height off the bottom: small and constant while in the viscous layer, and increasing in the geostrophic layer as it moves approximately horizontally and the bottom slopes away. The blue trajectory shows a second water parcel, starting directly above the first, near the top of the viscous boundary layer. It also follows a helical trajectory, but a smaller one, concentric with the red trajectory-never getting as close to the bottom or as far away.

These jets have one extremely odd feature: they are both banked against their respective left wall. Geostrophic currents tend to be found on the right wall when $f>0$. These jets are outside the viscous boundary layers, isopycnal, and are not externally forced, so they should be geostrophic. So what is going on?

The jets are best understood by looking at Lagrangian trajectories of water parcels in the viscous layer, such as the two trajectories in Figure 4-7. They start in the viscous bottom layer in the center of a canyon. The red water parcel starts at the heart of the the viscous layer, near where $v=v_{\max }$, less than $10 \mathrm{~m}$ off the bottom. The blue water parcel starts 
directly above it, about twice the height off the bottom. That height off the bottom is tracked by the shade of red or blue as they move along the canyon. The red water parcel begins by moving up the slope and to the right in the bottom layer. It stays the same low height off the bottom as it moves up the slope, having vertical but no slope-normal motion. Eventually, though, it moves far enough to the right that it hits the canyon sidewall. When this happens, there is nowhere for the water parcel to go but out of the bottom boundary layer. However, once it is outside the bottom boundary layer, it is no longer being forced by the isopycnal tilt next to the sloping bottom. In a stratified fluid without a source of potential or kinetic energy, it is constrained to move isopycnally. As it circulates back across the canyon, moving approximately horizontally, the bottom slopes farther and farther away, red getting darker and darker, and it is soon hundreds of meters off the bottom.

Something similar happens in the opposite direction near the other sidewall of the canyon. Water parcels moving up and to the right in the bottom layer near the left canyon wall mean that new water must be continuously entrained into the bottom layer, and it is expected to enter isopycnally. Our red water parcel therefore moves seamlessly and isopycnally from the outflow on the right wall to the inflow on the left wall, now getting closer and closer to the sloping bottom, the red getting paler and paler. It reenters the bottom layer at approximately the same along-canyon distance that it left, and once again starts moving upslope and to the right in the bottom boundary layer. All together, the water parcel takes a path that is approximately helix-shaped.

If we look at this same trajectory in terms of density (Figure 4-8), we can see the causes and consequences of such a path in a different way. When the water parcel is in the viscous bottom layer, it is constantly losing density through diffusive heat flux convergence at the bottom; it's upslope velocity is also a diapycnal velocity. However when it circulates outside the the bottom layer, it is in a uniform background stratification. Therefore diffusion is adding and removing density at the same rate. It is not forced and it is not changing mass, so it must move isopycnally, which here means approximately horizontally. The only water transformation, the only vertical motion, and the only net progress along the 


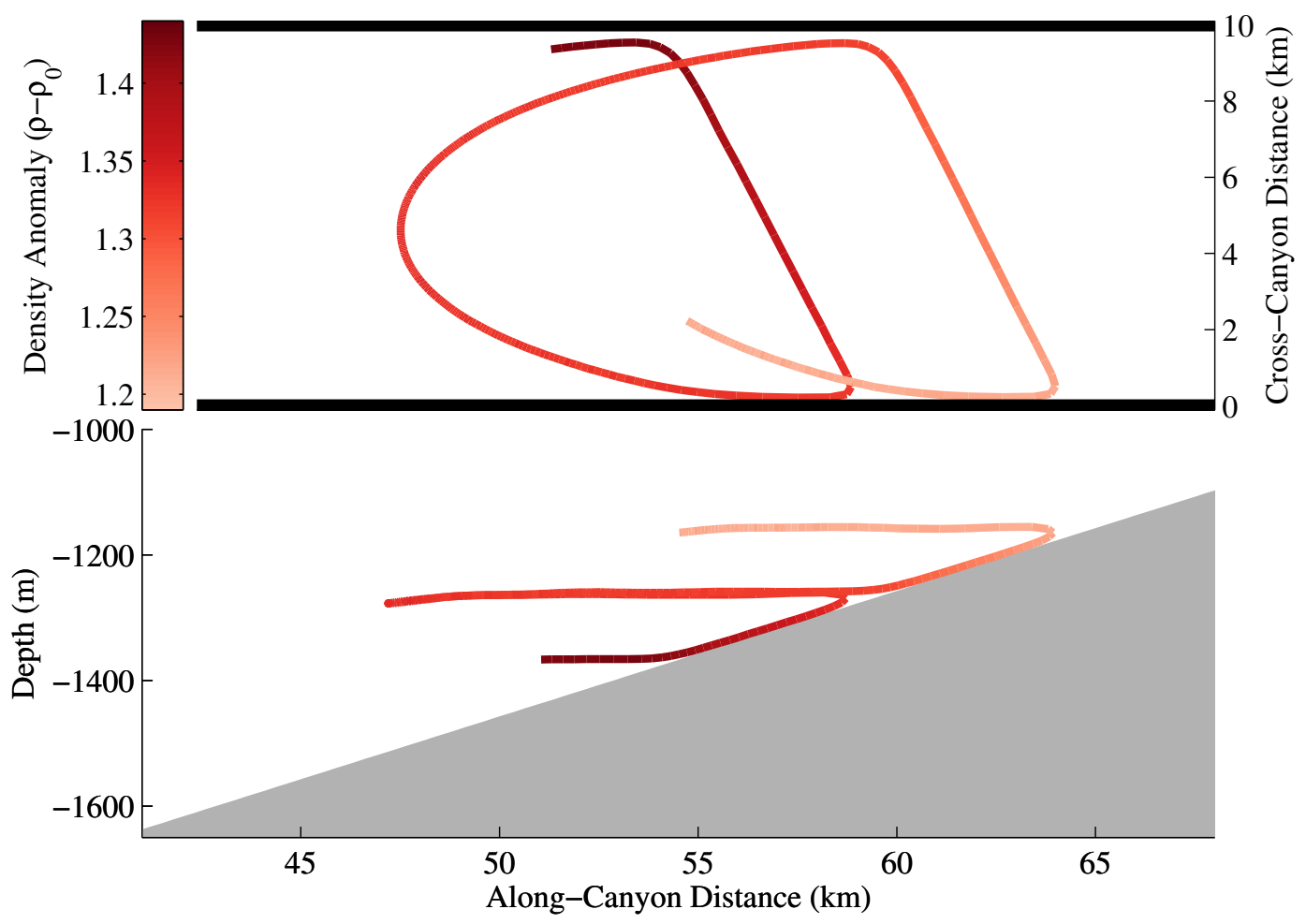

Figure 4-8: The density of a water parcel (given by the color) varies along the trajectory of the same water parcel shown in red in Figure 4-7. When the trajectory is viewed from the side (lower), we can see that the water parcel only moves diapycnally and changes density while in the bottom boundary layer. When circulating through the geostrophic boundary layer, it moves isopycnally, and so largely horizontally.

canyon happens to the water actually in the bottom layer. At the same time, all water parcels are constantly moving in or out of the bottom layer.

Returning to Figure 4-7, the blue water parcel begins higher in the boundary layer, and so follows a similar but somewhat more subdued path. Like the red water parcel, it follows a helical path, circling in and out of the viscous bottom layer as it moves along the canyon. Its helix is roughly concentric with the red water parcel, but smaller amplitude, never getting into the heart of the viscous layer, but never getting as far away from it either.

If we think about integrating millions of trajectories like those in Figures 4-7 and 4-8, many of the puzzling features of the Eulerian fields in Figure 4-5 become clear. The jets are simply water flowing out of the viscous layer on the right side of the canyon and into it 
on the left. This also explains why the viscous layer carries almost all of the along-canyon volume flux, and the flux in the up- and down-canyon jets cancels almost perfectly: the jets are feeding the viscous layer on the left and emptying it on the right. Since the viscous layer isn't changing as we move along canyon, the amount of water going into it must match the amount of water coming out.

There is a layer of water, much thicker than the viscous layer, that is always in the process of emerging from and reentering the viscous layer. Though it is closely interacting with the viscous layer, this larger outer layer is governed by geostrophic dynamics. This is the geostrophic layer I mentioned at the beginning of the section. This is a boundary layer with a well-defined scale thickness. If the horizontal size of the geostrophic circulation is set by the canyon width $L$, the geostrophic layer has thickness:

$$
\delta_{G}=L \tan \alpha_{0}
$$

For the simulations shown in Figures $4-5-4-8, \delta_{G}$ is on the order of $200 \mathrm{~m}$. In all of these figures, we can see this accurately reflects the vertical scale of the jets and the height off the bottom of the geostrophic excursions of Lagrangian trajectories. Since there is no heat flux convergence in the geostrophic layer, this thickness should be independent of the diffusivity, and it is.

This current structure in a canyon has two major consequences. First, the volume of fluid that is directly affected by the viscous bottom boundary layer is much larger than volume of the viscous layer itself. In the previous chapters, we discussed how introducing topography in the upslope direction can create exchange flows between the viscous boundary layer and the far field. Here we find that the canyon sidewalls don't just encourage exchange, they require it - all of the fluid in the geostrophic boundary layer is constantly being cycled through the viscous layer, and its water properties are transformed as it does. This means that a much larger volume of water is affected by the viscous boundary layer processes, but the effect on an individual water parcel is smaller, because each water parcel spends a relatively small percentage of its time in the viscous layer. However, this exchange 


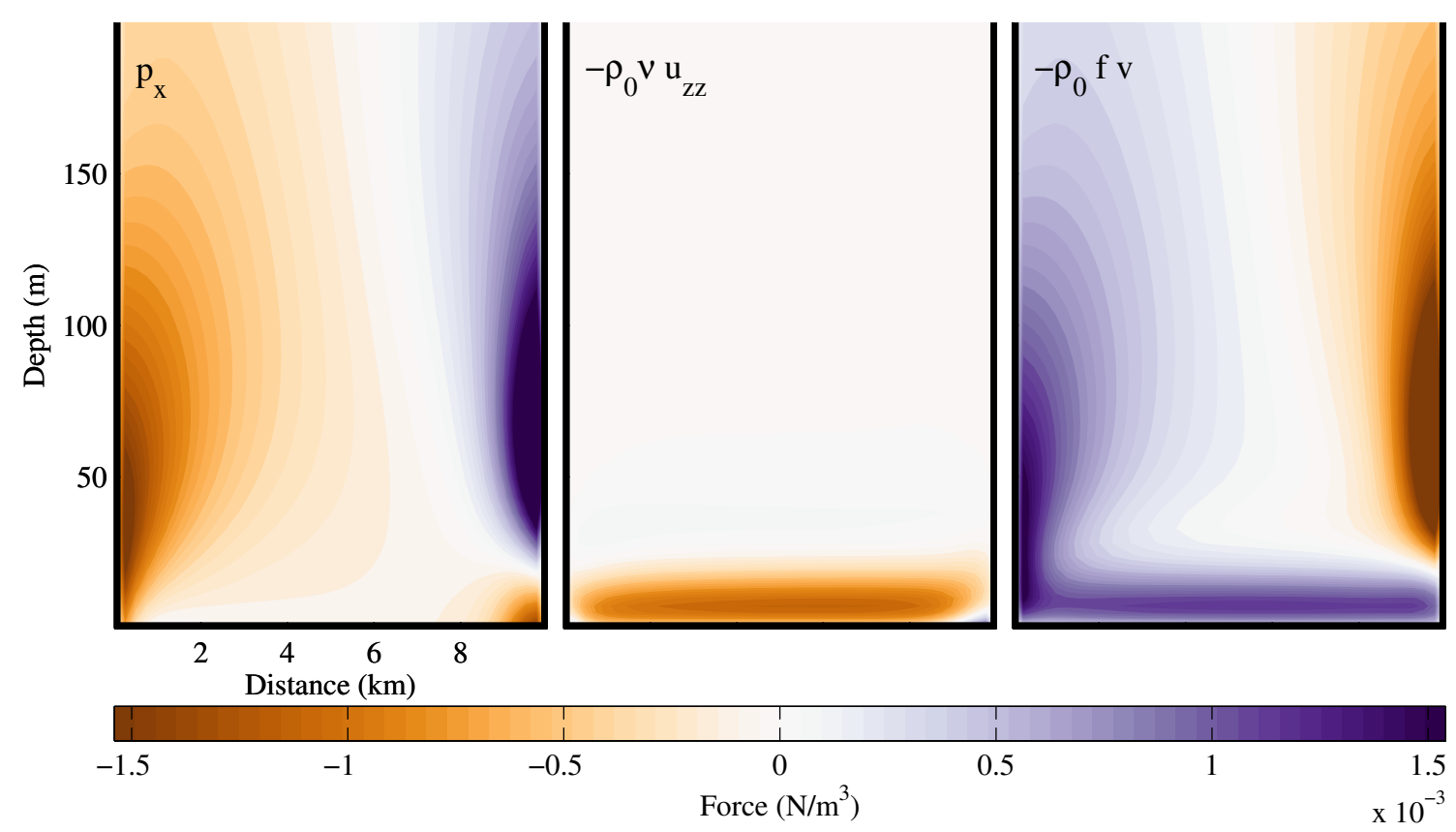

Figure 4-9: The dynamical regimes of the canyon circulation are clear in the along-isobath momentum balance. In the bottom boundary layer, the balance is between viscosity (center panel) and rotation (right panel). In the geostrophic layer, the balance is between pressure (left panel) and rotation (right panel). In the corner transition region, there is a three-way balance between pressure, viscosity, and rotation.

is unlike the exchanges seen in the two-dimensional studies because the same mass of water is cycling back and forth between the viscous and geostrophic layers. There isn't a finite volume of fluid that is sent into the far field, arbitrarily far from the bottom. The second major consequence is the relevant rate that fluid moves down the canyon is no longer the velocity scale of the bottom boundary layer.

In the next two subsections, I will develop a quantitative scaling for the sidewall jets in the geostrophic layer, allowing us to test our physical understanding and make quantitative predictions about the geostrophic layer's behavior. Then I will explore the issue of alongcanyon transport in greater detail. 


\section{4 .1 Dynamics of Sidewall Jets}

Understanding the momentum balance governing the sidewall jets allows us to make quantitative predictions about them. The most important quantity we would like to predict is the size of the sidewall jets. Without a reliable idea of scale, it would be impossible to observe them in the real ocean: we would not know where to look.

Each of the dynamical regimes described in the last section has its own primary momentum balance (Figure 4-9). In a two-dimensional viscous and diffusive bottom boundary layer like we studied in the previous chapters, the dynamical balance is between rotation and vertical viscous diffusion. Since the viscous layer is quite uniform across the width of the canyon, we expect that dynamical balance still holds in it, and the numerical simulations bear this out.

Outside of the bottom boundary layer, the velocity gradients are not large enough to create significant viscous dissipation, so we expect a geostrophic balance between rotation and pressure. Once again this is borne out by the numerical simulations. The pressure gradient arises from isopycnal tilt. Above the bottom boundary layer, the isopycnals are U-shaped, generating a pressure gradient toward the canyon center from both walls (Figure 4-5, bottom right). In the geostrophic layer, where the isopycnals tilt in the across-canyon direction, water parcels move isopycnally. This means they move approximately, not perfectly, horizontally. Since the geostrophic layer is baroclinic, there must be associated isopycnal tilt.

In between these two dynamical regimes, we expect to find the third dynamical regime identified at the beginning of this section: a transition region in which rotation, pressure, and viscosity are all important. This region occurs in the corners of the canyon, as shown in the cross-sections in Figure 4-9. This transition region is where the sidewall jets that we observed in our numerics form.

We can use this dynamical understanding to determine the scale of the sidewall jets. In 
non-dimensional form, the steady along-isobath (cross-canyon) momentum equation is:

$$
\underbrace{\varepsilon\left(u \frac{\partial u}{\partial x}+w \frac{\partial u}{\partial \eta}\right)}_{\text {advection }}-\underbrace{\frac{V \cos \alpha_{0}}{U} v}_{\text {rotation }}=\underbrace{-\frac{\partial p}{\partial x}}_{\text {pressure }}+\underbrace{E\left(\frac{\partial^{2} u}{\partial x^{2}}+\lambda^{2} \frac{\partial^{2} u}{\partial \eta^{2}}\right)}_{\text {viscosity }}
$$

$\varepsilon=\frac{U}{X f}$ is the Rossby number; $E=\frac{\nu}{f X^{2}}$ is the Ekman number; and $\lambda=\frac{X}{Z}$ is the aspect ratio of the flow. $X$ and $Z$ are the length scales in the $x$ and $\eta$ directions, and $U$ and $V$ are the velocity scales in the $x$ and $\xi$ directions. We expect that the Rossby number $\varepsilon$ and the Ekman number $E$ are small—for relevant parameter values, they are $O\left(10^{-2}\right)$ and $O\left(10^{-6}\right)$ respectively. However, the aspect ratio $\lambda$ is large, generally $O\left(10^{3}\right)$. Therefore we neglect the advection and the horizontal viscosity, but retain the vertical viscosity, as $E \lambda^{2} \sim 1$.

We can estimate the scale of the corner region using equation (4.11) and two different forms of the continuity equation. First, we have required that the boundary layer and associated dynamics do not vary in the up-slope direction. This means that:

$$
\frac{U}{X} \frac{\partial u}{\partial x}+\frac{W}{Z} \frac{\partial w}{\partial \eta}=0
$$

This should be true everywhere in the canyon. It implies that $\frac{U}{X} \sim \frac{W}{Z}$. In addition, outside the bottom boundary layer there is no forcing that can drive a vertical velocity-isopycnals are nearly flat and we have seen that flow is along isopycnals. Therefore, outside the bottom boundary layer, we find:

$$
\frac{U}{X} \frac{\partial u}{\partial x}+\frac{V_{g}}{Y} \frac{\partial v_{g}}{\partial y}=0
$$

The subscript $g$ indicates that this is the horizontal along-canyon velocity, perpendicular to gravity, not the along-slope velocity in slope-normal coordinates. Similarly, $y$ is the horizontal coordinate, distinct from the along-slope coordinate $\xi . u$ and $x$ are the same in both coordinate systems. This gives us a scaling $\frac{U}{X} \sim \frac{V_{g}}{Y}$. Because there is no variation in the along-slope direction, $v_{g}$ must be a projection of $w$ into the horizontal, so geometrically $V_{g}=V \cos \alpha_{0}$ and $Z=Y \sin \alpha_{0}$. In the corner region that forms the transition between the bottom boundary layer and the return flow in the far field, we expect equation (4.12) to be 
exactly true and equation (4.13) to be approximately true. That is, we are using geostrophic velocity scale, not the viscous velocity scales from the Phillips and Thorpe layers. The two continuity equations combine to tell us that:

$$
W \sim V \cos \alpha_{0} \sin \alpha_{0}
$$

If we now return to the momentum equation (4.11), the rotation/viscosity balance gives us:

$$
\frac{V \cos \alpha_{0}}{U} \sim E \lambda^{2}=\frac{\nu}{f Z^{2}}
$$

This implies that the corner region has the scale height of the Ekman layer. If we use the scaling from equation (4.12) to express $Z$ in terms of $U, X$, and $W$, then use equation (4.14) to express $W$ in terms of $V$ and $\alpha_{0}$, we can use this to get an along-isobath length scale in terms of the horizontal velocities:

$$
X^{2} \sim \frac{\nu}{f \sin ^{2} \alpha_{0}} \frac{U^{3}}{V^{3} \cos ^{3} \alpha_{0}}
$$

In this transition region, however, we expect not just a rotation/viscosity balance, but a three-way balance between rotation, viscosity, and pressure. From equation (4.11), we can see that the pressure/rotation balance implies $\frac{V \cos \alpha_{0}}{U} \sim 1$. This gives a final scale for the width of the transition region:

$$
X \sim \sqrt{\frac{\nu}{f \sin ^{2} \alpha_{0}}}
$$

The full primitive-equation numerical simulations confirm this scaling for values of the slope Burger number $S$ that vary by almost a factor of 100 (Figure 4-10). The accuracy of this scaling gives us confidence in the general picture of viscous and geostrophic layers I have described. It also allows us to predict dynamics in potential observing sites using only a relatively crude knowledge of the canyon topography. 


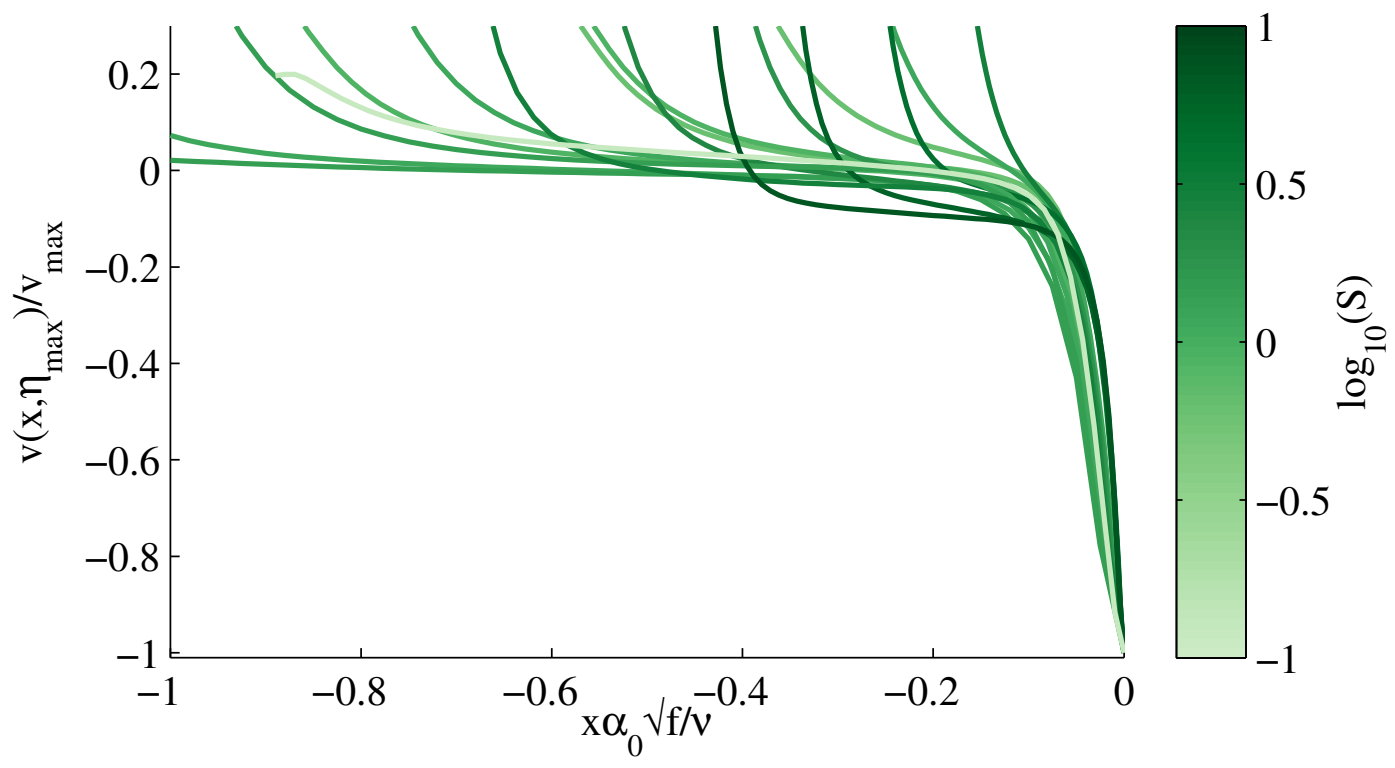

Figure 4-10: Over almost 100 times variation in the slope Burger number $S$, the structure and thickness of the sidewall jet scales consistently with $X \sim \sqrt{\frac{\nu}{f \sin ^{2} \alpha_{0}}}$. This scaling arises from a three-way dynamical balance between rotation, pressure, and vertical viscosity. Each of the 17 traces in this figure shows the along-canyon velocity in the sidewall jet of fluid exiting the bottom boundary layer. Each is normalized to a peak velocity of 1 , and each comes from an independent numerical simulation, where $0.01<\alpha_{0}<0.05$ and $2 \times 10^{-5} \mathrm{~s}^{-1}<f<5 \times 10^{-4} \mathrm{~s}^{-1}$. The traces turn upward as they approach the left wall of the canyon in the rescaled cross-canyon coordinate $x / X$.

\section{4 .2 Along-Canyon Motion in Time}

The flow structure described in this section - a viscous bottom layer, an overlying geostrophic layer, and a constant circulation of water between the two- has important consequences for the motion of water parcels in time and transport along-canyon. In the two-dimensional case, the relevant transport velocity for water in the boundary layer was the velocity scale for that boundary layer. However, water parcels move at that rate-generally around 1-2 $\mathrm{cm} / \mathrm{s}$ in the parameter ranges we care about—only when they are actually in the viscous bottom layer. Since water is constantly moving between the bottom layer and the geostrophic layer, and in the geostrophic layer water parcels have little or no net transport down canyon, the time-averaged along-canyon velocity of a water parcel is much less than the velocity 
in the bottom layer. This means that tracers, nutrients, and anything else that follows the water will move at a much slower average rate than we might naively expect based on the two-dimensional dynamics we explored in the previous two chapters.

We can see this effect directly by looking at the along-canyon position as a function of time for the Lagrangian trajectories we studied earlier in this chapter (Figure 4-11). These positions are shown for 16 water parcels that begin in a single column that spans the thickness of the viscous layer. As we saw in Figure 4-7, the water parcels that start lower in the boundary layer make fewer, larger circulations through the geostrophic layer and water parcels that start higher make a larger number of smaller circulations, but they all move along canyon at approximately the same average rate, $21 \pm 3 \mathrm{~km} \mathrm{yr}^{-1}$, compared to approximately $400 \mathrm{~km} \mathrm{yr}^{-1}$ given by the upslope velocity in the viscous layer.

There are two noteworthy aspects of this. First, as expected, the time-averaged Lagrangian velocity is much slower-20 times slower-than the viscous layer suggests. Second, all the water parcels are moving at about the same rate. This means water parcels do not move permanently or infinitely far away from one another as they move along the canyon. Irreversible mixing is associated with fluid trajectories diverging in space and time, so the geostrophic layer does not introduce any new mixing. In a real ocean canyon, where the sidewall currents are moving along rough topography, we would expect the situation to be much more complicated, but it is important to note that the geostrophic layer does not introduce a new kind of mixing mechanism.

The reduced time-averaged Lagrangian velocity shown in Figure 4-11 may help to explain a seeming contradiction between two different types of data collected during the BBTRE between 1996-2000. In this experiment, an inert tracer was injected at approximately $4000 \mathrm{~m}$ deep in the Brazil Basin, near $22^{\circ} \mathrm{S}, 18^{\circ} \mathrm{W}$. After 14 months, Ledwell et al. [2000] observed that the tracer patch had split into two patches, the smaller of which had migrated east along isopycnals into the Mid-Atlantic Ridge flank canyon at $22^{\circ} \mathrm{S}$. One year later, 26 months after injection, the tracer patch in the $22^{\circ} \mathrm{S}$ canyon had grown to include $30 \%$ of all the tracer and had spread diapycnally dramatically, but on average it had not 


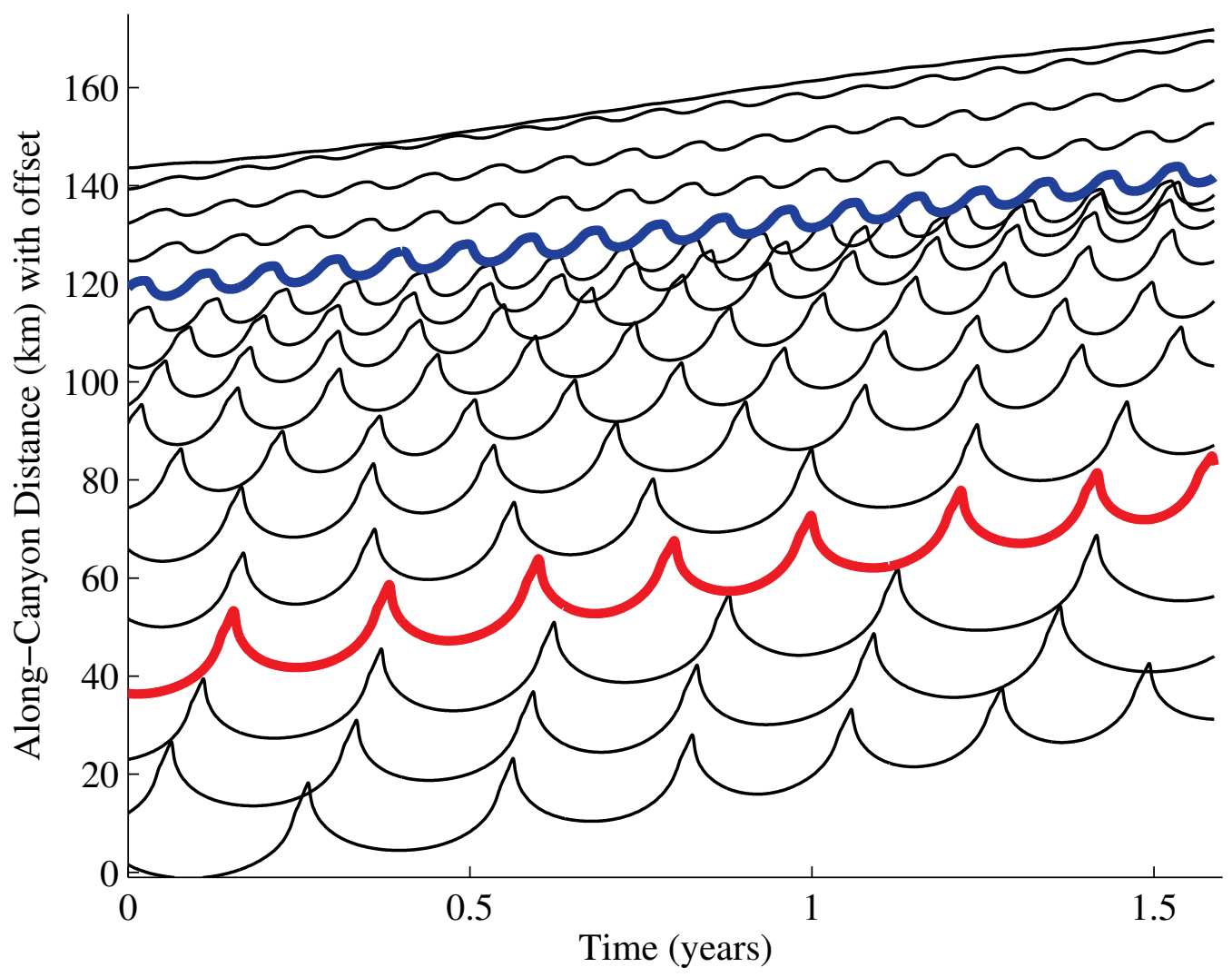

Figure 4-11: Water parcels that start at many different heights within the boundary layers have similar average rates of along-canyon motion. Here the along-canyon position as a function of time is plotted for 16 water parcels that began in a single column spanning the thickness of the viscous boundary layer. The traces are vertically offset from one another for clarity. Water parcels lower in the boundary layer make fewer larger circulations through the geostrophic layer (which give the traces their cycloidal shape), and those higher in the boundary layer make more smaller turns. However, all traces have a similar mean slope, so all water parcels have a similar mean rate down the canyon, $21 \pm 3 \mathrm{~km} \mathrm{yr}^{-1}$ for $\alpha_{0}=0.02$ and $f=10^{-4} \mathrm{~s}^{-1}$, compared to approximately $400 \mathrm{~km} \mathrm{yr}^{-1}$ given by the upslope velocity in the viscous layer. The red and blue traces are from the trajectories shown in red and blue in Figure 4-7.

moved significantly in space. The authors mention both that particulate effects may have caused the tracer to migrate to denser water classes and that the enhanced diffusion in the canyon may have mixed the tracer back to the original injection density, but overall the tracer in the canyon did not seem to move significantly in density between 14-26 months. The relevant figure from the paper, showing maps of the tracer distribution, is reproduced 
in Figure 4-12. Also as part of the the BBTRE, a current meter mooring was deployed in the $22^{\circ} \mathrm{S}$ canyon, slightly to the east of the injection site. Over a two year deployment, the mooring measured a bottom-intensified mean eastward velocity inside the canyon of 1.5-2 $\mathrm{cm} \mathrm{s}^{-1}$ [Toole, 2007]. The mooring was deployed in 1998, after the tracer data was collected, but we have no reason to believe that both the tracer and the mooring measurements do not represent the average conditions in this canyon.

These two types of data do not seem fully consistent with one another. $1.5 \mathrm{~cm} \mathrm{~s}^{-1}$ is about $500 \mathrm{~km} \mathrm{yr}^{-1}$, so between the two tracer surveys shown in Figure 4-12, we might expect the tracer to move $500 \mathrm{~km}$ - about 5 degrees of longitude — to the east. It did not. The boundary layer mechanism described here, where water is cycled in and out of a relatively thin boundary layer, so the time averaged Lagrangian velocity in the canyon is much smaller than the boundary layer velocity, might explain this inconsistency. In the idealized simulations, the transport velocity of the combined viscous and geostrophic layers could be less than $5 \%$ the velocity of the viscous layer. That effect is more than large enough to make this inconsistency disappear.

In this section, I have presented a thorough description of the dynamics in an unforced stratified fluid in a canyon or channel with a sloping bottom. The presence of the sidewalls leads the viscous bottom boundary layer to drive a much thicker circulating geostrophic boundary layer that manifests in the Eulerian velocity fields as two jets, one going up the canyon and one going down. Contrary to our expectations for geostrophic currents, these jets are banked against their respective left walls, but that can be understood by thinking about Lagrangian trajectories in the viscous and geostrophic layers, which tend to follow approximately helical paths up the bottom slope of the canyon. We can accurately predict the scale of these jets over two orders of magnitude for the slope Burger number. This circulation geometry means the transport along the canyon is much slower than the viscous layer velocity scale suggests, which is important for nutrients and tracers, and may help us explain some observations from the BBTRE.

From here we will move on to look at canyons with simple varying bottom topography, 

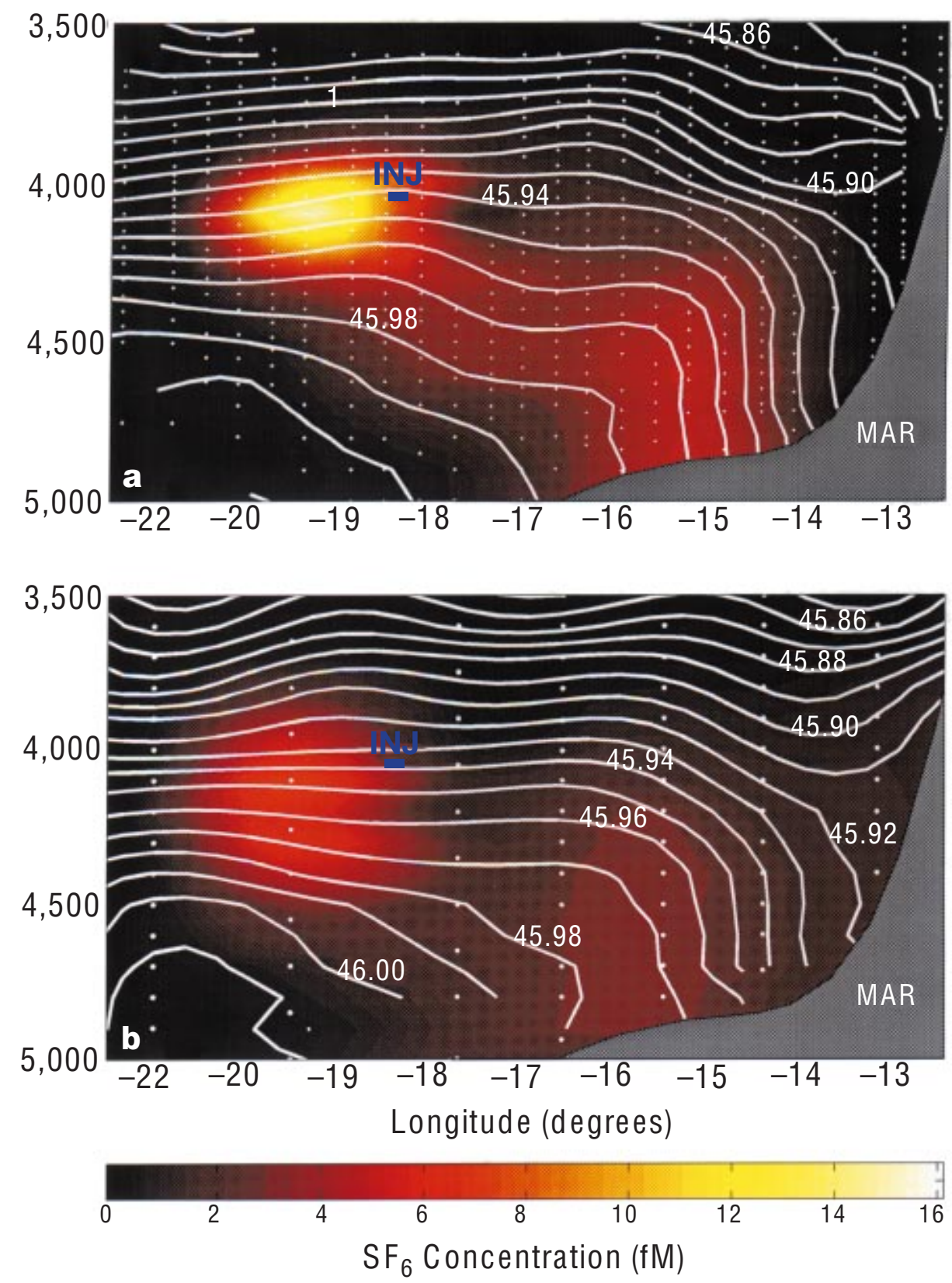

Figure 4-12: The inert tracer injected in the BBTRE moved up canyon much more slowly than the current meter deployed at $18 \mathrm{~W}$ would imply. After moving isopycnally from the injection site (labeled INJ), the above figure, reproduced from Ledwell et al. [2000], shows little discernible westward motion of the dye between 14 months after release (top) and 26 months after release (bottom). The mean velocity from the current meter would imply that the dye move about $500 \mathrm{~km}$ to the east, or about 5 degrees longitude [Toole, 2007]. 
and its implications for mixing and exchange with the far field.

\subsection{Circulation in a Canyon with Sills}

Typical mid-ocean ridge flank canyons are regularly obstructed by sills that block the full width of the canyon. These sills are usually $100-500 \mathrm{~m}$ tall and 3-8 km wide. These sills are the subject of heightened interest because they may be the sites of small-scale overflows, strong interactions between the bottom boundary layer and the topography, and enhanced mixing. We saw many examples of these dynamics in the two-dimensional studies in the previous chapters. To start to understand some of the effects of these sills on the circulation and fluid dynamics of an ocean-bottom canyon, I introduced isolated sills of varying heights into the idealized canyons we analyzed in the previous section.

The four simulations in Figure 4-13 show identical canyons with a single sill $8 \mathrm{~km}$ wide and of four different heights, spanning the three bottom boundary layer geometries described in Chapters 2 and 3: (a) linear, (b) overflow, (c) and (d) reversing. The four sills also span a range of scales with respect to the geostrophic layer. In this example, the geostrophic layer is $200 \mathrm{~m}$ thick, so the sills—at $40 \mathrm{~m}, 100 \mathrm{~m}, 200 \mathrm{~m}$, and $400 \mathrm{~m}$ tallrange from considerably smaller than the geostrophic layer to twice its height.

Each plot in Figure 4-13 shows 16 trajectories, which start more than $30 \mathrm{~km}$ upstream of the sill in an array that spans the thickness of the bottom boundary layer in the central $5 \mathrm{~km}$ of the $10 \mathrm{~km}$-wide canyon. Away from the sill, the trajectories completely cover the thickness of the geostrophic layer, given in equation (4.10), and shown as a short yellow line in Figure 4-13a. For a very small amplitude sill, the viscous boundary layer is modulated largely as we saw in the two-dimensional studies. There is no discernible cumulative effect on the geostrophic boundary layer, though the trajectories of particular water parcels are affected by the sill.

As the sill height is increased, more and more fluid is exchanged between the far field and the geostrophic and viscous layers. The portion of trajectories in the figure that leave 

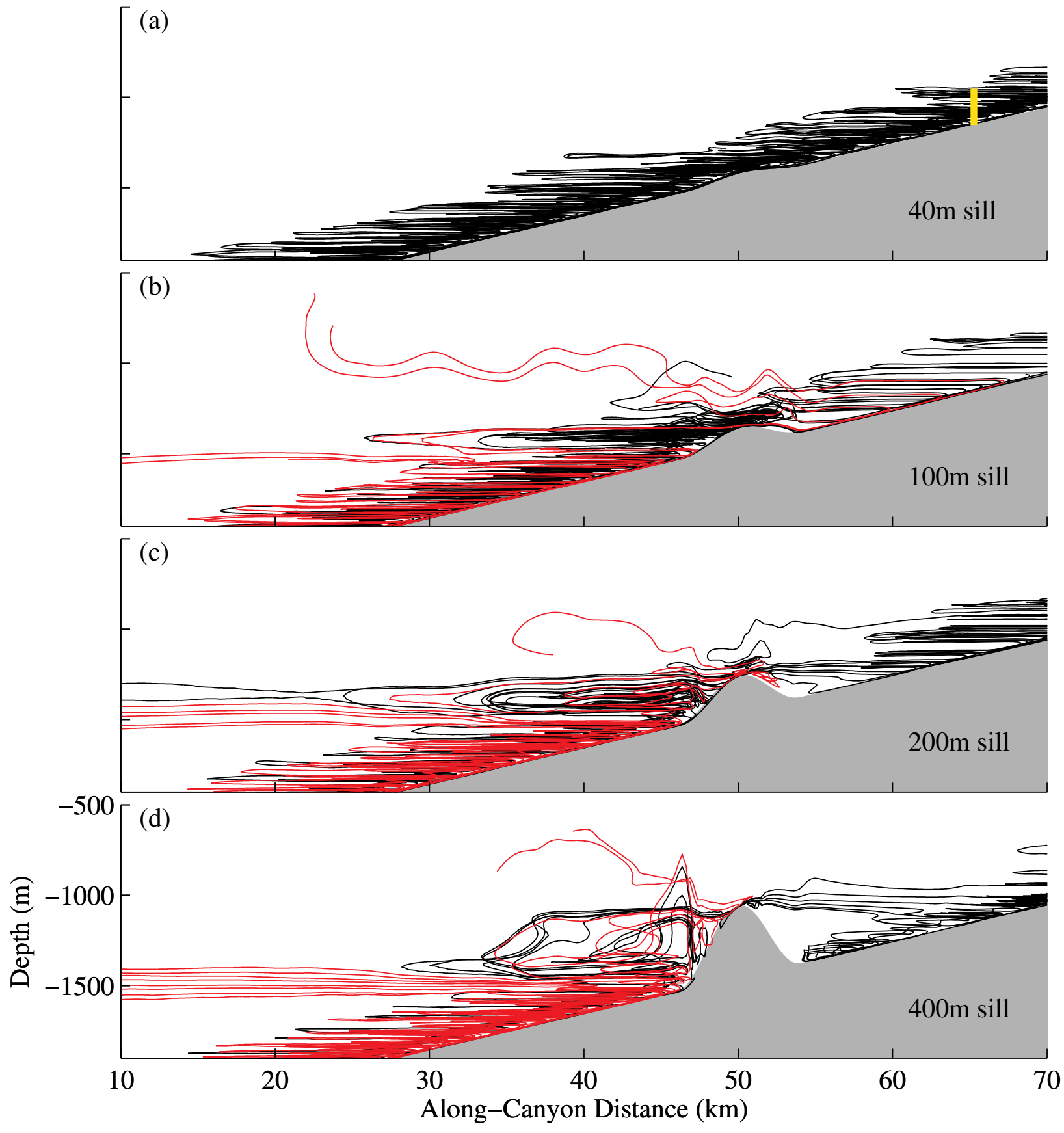

Figure 4-13: Larger sills cause more exchange with the far field. The black traces show trajectories that remain in or near the geostrophic layer, and the red traces show trajectories that enter the far field. The short yellow line in the boundary layer in subplot (a) shows the predicted thickness of the geostrophic boundary layer from equation (4.10); as expected, it matches very closely with the region that is filled with Lagrangian trajectories. The large vertical excursions of a few trajectories in the far field do not indicate substantial vertical velocities; these trajectories are five or more years long and the velocity is not significantly different from zero. 
the boundary layers entirely is $0 \%, 25 \%, 50 \%$, and $63 \%$, respectively. Fluid leaves the boundary layers through two distinct but related mechanisms, both resulting from variations in the topography. First, changes in slope change the local transport in the bottom boundary layer as predicted in equation (4.9), so water is forced out of or entrained into the boundary region. This can be seen in the horizontal outflowing trajectories at about $1500 \mathrm{~m}$ depth in Figure 4-13b, c, d. This is exactly the mechanism that we saw in the studies of broken slopes and small-amplitude topography in Chapter 2. Second, larger sills with the upslope flows on both faces have a flow convergence at the peak, and water must leave the boundary layer vertically. Most of this water will be re-entrained into the boundary layers nearby, but some of it will move into the far field. Close to varying topography the viscous and geostrophic boundary layers associated with different portions of the slope can overlap and interact, so the vertical velocity at the peak of the sill can also eject water parcels that did not come up to the peak directly through the bottom layer. For example, in Figure $4-13 b$, the two trajectories that leave the boundary layers at the sill peak both crossed the sill, entered the viscous bottom layer downstream of the sill, then while they circulating through the geostrophic layer in the trough they were entrained into the water leaving the boundary layers at the sill. Once in the far field, the water parcels' velocity is not significantly different from zero. The large vertical excursions seen in some trajectories in the far field result from insignificant velocities integrated over many years, not from significant diapycnal fluxes.

Unlike the canyon with a constant-slope bottom, the sills cause exchange not just between the viscous and geostrophic layers, but actually exchange water with the far field. The red trajectories in Figure 4-13 are never re-entrained into any boundary layer anywhere in the domain. This is more similar to the non-rotating two-dimensional case than the rotating case, because without an along-isobath flow far from the boundary, there is no opportunity for the complicated interactions between rotational (Ekman) and buoyant (Phillips) effects. As with both the rotating and non-rotating two-dimensional cases, though, these exchange flows can affect the stratification far from the boundary because the exchange is 
anchored in space by the topography.

Interestingly, the exchanges between the boundary layers and the far-field are concentrated on the left wall of the canyon (Figure 4-14a). On the right wall of the canyon (Figure 4-14b), the pumping from the viscous to the geostrophic layer is modulated by the local slope, but no velocity is induced outside the geostrophic layer. We can see the reason for this looking at a trajectory that exits the boundary layers (Figure 4-14c). This is an extremely common trajectory. A water parcel moves in the standard helical path as it approaches the sill (shown in grey shading). After leaving the viscous layer near the sill on the right wall, it is unable to reenter the viscous layer on the left wall because the slope of the sill is steeper than the background. This means the volume flux, proportional to $\cot \alpha$, is lower. With nowhere else to go, the water parcel eventually enters a pressure-driven flow into the far field. Higher up on the sill, where the viscous layer is now increasing its flux, its usual entrainment on the left wall must be augmented by fluid from the far field. In a canyon, we have have the possibility for laterally varying dynamics, and this means that unlike the two-dimensional case, we do not expect the water in the troughs between sills to completely homogenize with time. It can remain stratified because denser water from upstream is advected over along the left wall and transported by the viscous boundary layer toward the right.

Topography not only communicates the boundary layer dynamics to the far field, it can also enhance mixing in the boundary layers themselves. We can see how this works by looking at the trajectories of two water parcels that begin adjacent to one another upstream of a sill, but become separated at the sill (Figure 4-15). They begin near each other in the heart of the viscous boundary layer and follow similar trajectories while the canyon bottom has a constant slope. As they approach the sill $30 \mathrm{~km}$ from where they began, they are still side-by-side. They are in the viscous boundary layer as they near the top of the $100 \mathrm{~m}$-tall sill obstructing the canyon. One water parcel manages to cross it, while the other is forced into the geostrophic boundary layer still upstream of the sill. That water parcel does an extra circulation through the geostrophic layer (indicated in Figure 4-15a 


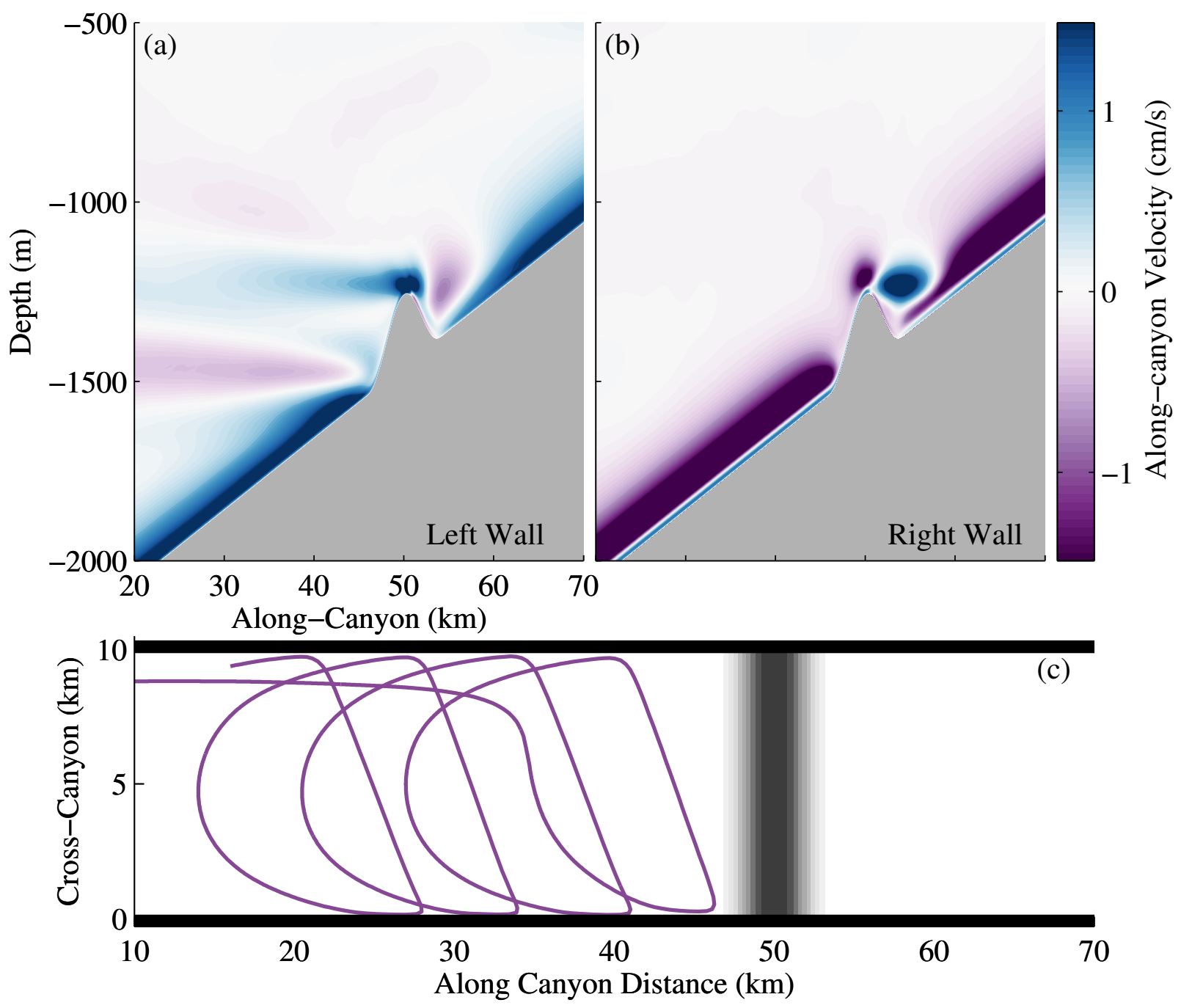

Figure 4-14: Water is exchanged between the boundary layers and the far field along the left wall (a), in the region where water enters the viscous layer from the geostrophic layer, as shown in this plot of along-canyon velocity. Along the right wall (b), where water is leaving the viscous layer, even substantial sills do not induce any velocity in the far field. Looking at a typical trajectory for a water parcel that moves to the far field (c), we can see how it leaves the viscous layer on the right side of the canyon upstream of the the sill (shown in grey shading), but cannot reenter the viscous layer on the left side because the volume flux in the viscous layer is much lower in the steeper section. Blocked by the sill, the water has nowhere else to go but into the far field along the wall.

with an arrow) before reentering the viscous layer and crossing the sill. The various flow reversals and recirculations generated by the sill cause the two water parcels to meander a 
bit, but eventually they both enter the viscous bottom boundary layer downstream of the sill at almost exactly the same point. From there, their trajectories remain spatially close until the end of the domain. However, since one water parcel made an extra loop through the geostrophic boundary layer, it is now more than eight months behind the other water parcel. The times that the two reenter the viscous layer downstream of the sill are marked in Figure 4-15b. Even though the two water parcels follow similar paths, they do not remain close to one another as they would in a canyon whose bottom had a constant slope-they can become widely separated in time.

The mechanism for additional mixing that we saw with these two trajectories shows up clearly in the statistics of the distance between pairs of trajectories. In a turbulent fluid with classical Fickian diffusion, we can relate the separation between particles to diffusion. These flows, however, are laminar, highly ordered, and steady in time. We should not expect Fickian diffusion to be directly applicable. The basic idea that larger particle separations indicate stronger mixing is a robust one, though. To see how the particle separation was affected by the presence of topography, I calculated 525 trajectories in each canyon, so at any point in time, there as many as $525 \times 524=2.75 \times 10^{5}$ pairs of particles with known separations, and $>2.5$ million pairs over the full time span.

When binned and plotted as a function of their along-canyon position, the effect of the topography on water parcel separation is clear (Figure 4-16). Where the canyon bottom has a constant slope-in the canyons without sills, or upstream or downstream of a sill-the separation between particles increases slowly as the particles move along the canyon. Since water parcels move along the canyon at a steady mean velocity in these areas (cf. Figure 4-11), it's probably safe to see along-canyon position as a rough stand-in for time. The most noteworthy thing in Figure 4-16, though, is what happens at the sill. There the average particle separation jumps, and higher sills produce bigger jumps. Even in in this laminar, low Reynolds number flow, presence of a single, isolated variation in bottom topography produces the effect of an isolated burst of mixing. If we imagine a real canyon with continuously varying topography, this mixing increase might be happening everywhere. 

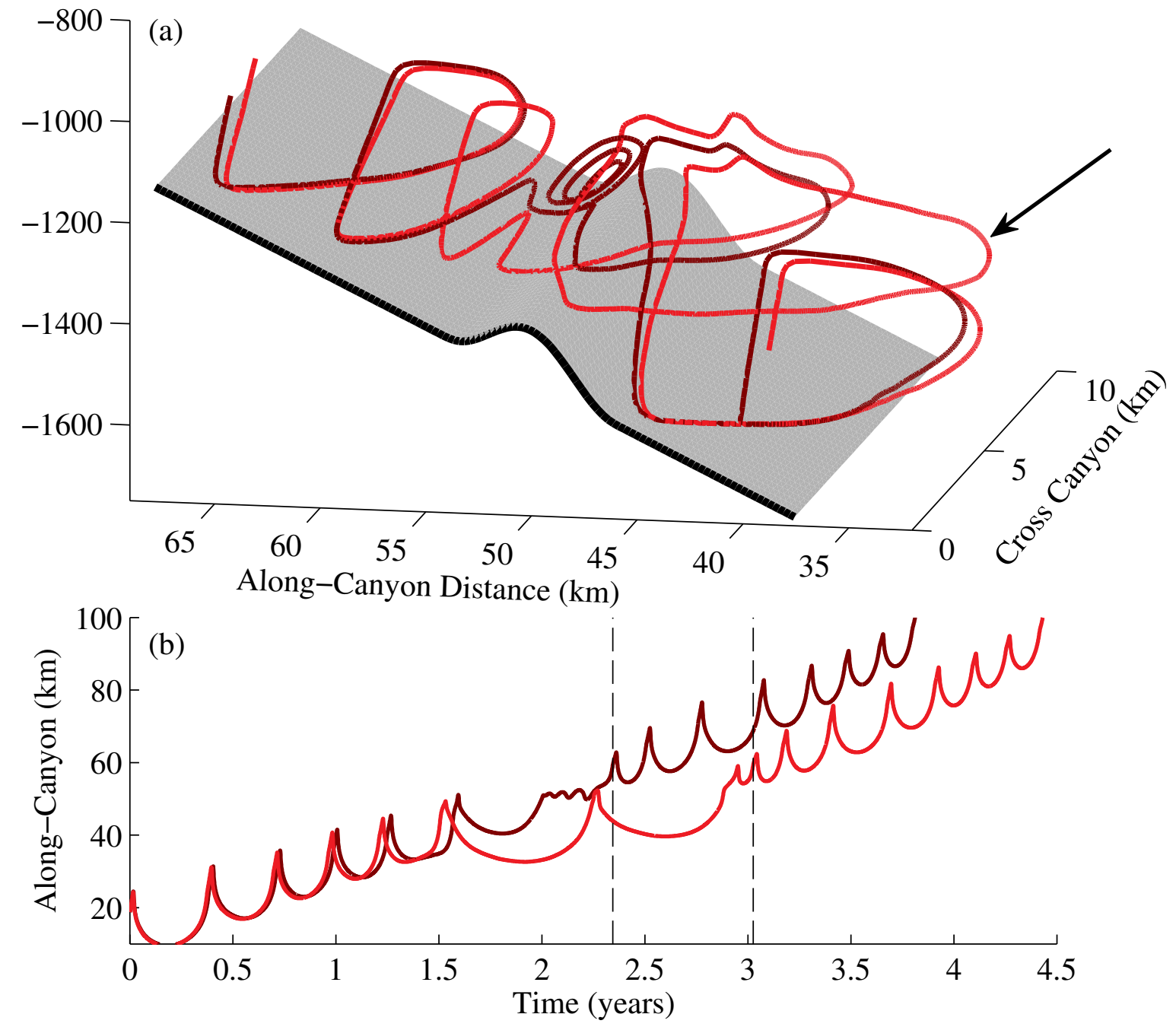

Figure 4-15: Topography can separate adjacent water parcels in both space and time. Two trajectories start next to one another, but as they approach the sill one is able to cross (dark red), while the other (light red) does an extra circulation through the geostrophic layer (indicated with an arrow) before making to the other side. While they both reenter the viscous layer at similar point downstream of the sill, if we look at their motion in time (lower plot), we can see that the second trajectory is now eight months behind the firstthe black dashed lines show where they both reach the same point in space.

In sum, introducing simple, isolated topography into a canyon with a sloping bottom, produces a variety of interesting effects. First, it is able to increase mixing, seen in these low Reynolds number simulations through the divergence of Lagrangian trajectories. The 


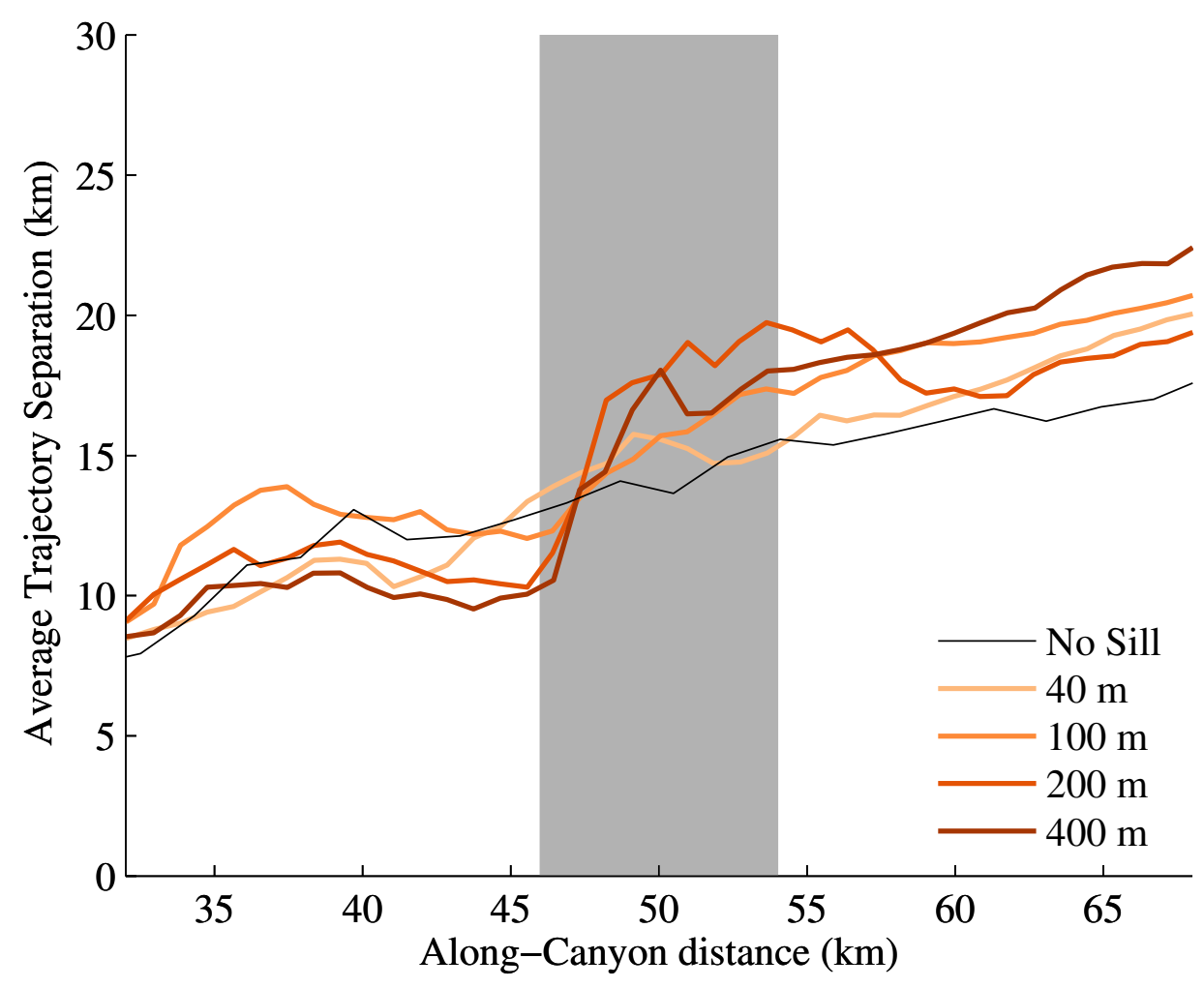

Figure 4-16: The average separation between trajectories jumps up at the sill, meaning that topography generates mixing even in low Reynolds number flows. Where the canyon bottom has a constant slope, the mean distance between the Lagrangian particles increases at an approximately constant rate. However, the separation jumps much more rapidly at the sill, whose position is shaded in grey. The biggest jumps happen in canyons with the largest sills; larger topography creates more mixing. The variance of the trajectory separation is large: $\sigma=5 \mathrm{~km}$. 
average separation between trajectories increases faster over taller sills than over shorter ones. Second, in addition to increasing mixing, it is able to communicate that enhanced mixing to the ocean basin: it can force exchanges of water beyond the geostrophic layer and to the far-field. These exchanges are also stronger with taller sills, and tend to be concentrated along the left wall of the canyon.

\subsection{Conclusions}

This work has given the first description of the surprisingly rich dynamics that arise from a very simple setting: a channel with a sloping bottom, filled with a stratified fluid. The dynamics are driven by the same basic mechanism that we have been exploring throughout this thesis: over a sloping bottom, the no-flux bottom boundary condition causes isopycnals to bend down so as to intersect the bottom at a right angle. This, in turn, generates a horizontal pressure gradient that drives an upslope flow. This flow is then able to compensate for the heat/salt flux divergence at the bottom boundary from the background density gradient.

From these rather humble but also strangely elegant ingredients, we are able to produce a hearty meal of interesting flow behavior. Because the canyon walls do not affect the thermal constraints on the flow, we know that the boundary layer here must carry the same volume flux as the two-dimensional boundary layer, but it does it in a very different way. Instead of just two dynamical regimes-a viscous bottom boundary layer and the inviscid far field-the canyon has four. Between the viscous layer and the far field, there is a geostrophic layer where water that is forced out of the viscous layer on the right wall of the canyon recirculates and reenters the viscous layer on the left wall. The walls do not just encourage fluid to be exchanged in and out of the viscous layer, they require it. This gives water parcels an approximately helical trajectory up the canyon: moving through the bottom viscous layer, exiting at the right wall, circulating slowly back across the canyon isopycnally in the geostrophic layer, and reentering the viscous layer. 
Integrated oven the whole canyon, this motion leads to the odd appearance of geostrophic jets banked against their respective left walls, and a large-scale anticyclonic circulation in the canyon, which can be even stronger and faster at its peak than the viscous layer. The basic dynamical balance of these jets is a three-way balance between rotation, pressure, and vertical viscosity, which in turn provides a scaling for the jets that we were able to validate over a variation of almost 100 times in $S$, the slope Burger number.

Introducing topography further complicates the dynamics, in ways that have many direct analogies to the two-dimensional cases studied in previous chapters. The effects of topography may be more closely analogous to the non-rotating two-dimensional boundary layers than the rotating case. The complicated interplay between the Ekman and the Phillips layer that dominated the physics of the two dimensional rotating case were absent here because the canyon walls prevented along-isobath currents from developing far from the boundary. Instead we saw the direct exchanges between the boundary layer and the far field of the non-rotating case, and as in that case, the flux constraint from equation (4.9) $Q=\kappa \cot \alpha$-was invaluable in understanding the location and size of the exchanges. As in the non-rotating case, we saw that topography in a canyon can both increase mixing and efficiently communicate that enhancement to the far field.

Perhaps most exciting, the work in this chapter finally provides some rich ideas that observers may be able to test in the real ocean. Most obvious, do we see any evidence of the sidewall jets and generally anti-cyclonic circulation in real canyons? If the jets are present, are they primarily geostrophic? These are questions whose answers can be known. The published data from the BBTRE suggest another possible observational test. The helical canyon circulation we described implies that the along-canyon transport of water parcels is much slower-perhaps 20 times slower-than the velocity in the viscous bottom boundary layer would suggest. In the BBTRE, passive tracer moved along canyon much more slowly than a nearby current meter mooring would suggest. Perhaps a more detailed study of the tracer motion with these physics in mind would yield evidence of these boundary layer processes. More generally, it would be interesting to ask if passive tracers 
move along-canyon more slowly than the mean velocity in the bottom boundary layer in other contexts.

However, we also have to remember the difficulties of observational comparison. This kind of viscous boundary layer has never been directly observed. It is only tens of meters thick, and potentially found thousands of meters below the surface. The boundary layer that we do see in abyssal canyons is considerably thicker than the viscous layer in the numerical simulations. In observations, isopycnals bend down toward the bottom over a thickness of about $500 \mathrm{~m}$, closer in thickness to the geostrophic layer (where isopycnals do not bend in the along-canyon direction) than the viscous layer. This scale may very well be set by the size of the variations in topography themselves, meaning that interactions between adjacent sills and with continuously varying topography are likely of first order importance.

These types of caveats, though, are simply what we all must live with as scientists seeking to understand the earth system - it will always be more complicated than our ideas. Nonetheless, this work has offered a new understanding of the physics of boundary layers in non-uniform geometries, while also offering opportunities for direct testing and comparison with ocean observations.

\section{A Appendix - Studying the Canyon Circulation in a Laboratory}

It may be possible to recreate in the laboratory the features of the canyon circulation described in section 4.4. Unlike the experiments proposed to test the non-rotating circulation in Chapter 2, the goal of these experiments would be to create and visualize the geostrophic boundary layer, not to visualize the details of the bottom boundary layer. Therefore, it is advantageous to generate a thinner bottom boundary layer and narrower sidewall jets, in order accommodate the geostrophic layer in a conveniently-sized tank.

The strongest constraint on such a laboratory experiment is likely to be size of the canyon that is feasible to construct. A standard geophysical fluid dynamics laboratory has 
a $1 \mathrm{~m}$ turning table, permitting values for the Coriolis parameter $f$ in the range $0.1-2 \mathrm{~s}^{-1}$. As discussed in the non-rotating laboratory experiments (Section 2.C ), the minimum angle where we are confident of generating an Phillips-type boundary layer is approximately $\alpha=5^{\circ}$. With the molecular viscosity of water $\nu=10^{-6} \mathrm{~m}^{2} \mathrm{~s}^{-1}$ and a Coriolis parameter $f=2 \mathrm{~s}^{-1}$, this gives a sidewall layer thickness of $X=7 \mathrm{~mm}$, from equation (4.17). In order to allow the sidewall jets on each side of the canyon to develop freely, we would like the canyon width to be $20-30$ times $X$, or $W=15-20 \mathrm{~cm}$. In each passage through the bottom layer, the water parcel moves along-canyon a distance of about $W V / U$, where $U$ and $V$ are the scale velocities of the along-isobath and along-canyon velocities in the bottom layer, taken from the two-dimensional rotating solution of Thorpe [1987]. For the above parameter values, $V / U$ is about 0.6 . To have space in the canyon for a water parcel to make a few circulations through the geostrophic layer, the canyon length should be at least $5 W V / U$, or at least $50 \mathrm{~cm}$ (Figure 4-17). The experiments could test a variety of values for $f, N$, and $\alpha$, but since $f$ and $N$ will both be order 1 , small angles will always produce small slope Burger number $S$. Larger slopes would be interesting, but would be far outside the parameter range of the modeling study presented here.

The flow could be visualized using dye, as in Peacock et al. [2004]. In lieu of the dye reservoir they used, the dye could be injected at a small number of isolated locations in the bottom boundary layer. We would then expect to see dye streaks following water parcel trajectories like those shown in Figures 4-7 and 4-8. We could reconstruct the threedimensional water parcel trajectory by filming the dye streaks from multiple angles.

The largest problem is likely going to be maintaining the stratification in the tank for long enough for the trajectory to develop clearly. The bottom boundary layer will only be about $\delta=1 \mathrm{~mm}$ thick, so the diffusive timescale for it is $T_{B L} 5-6 \mathrm{~min}$. The inertial timescale is about a second, so overall we expect the velocity field to be established quickly. However, it generally takes an hour or more for a tank filled with water to reach solid-body rotation, during which time the stratification will likely erode significantly. It might be possible to address this by spinning up the tank and then lowering in the sloping bottom 


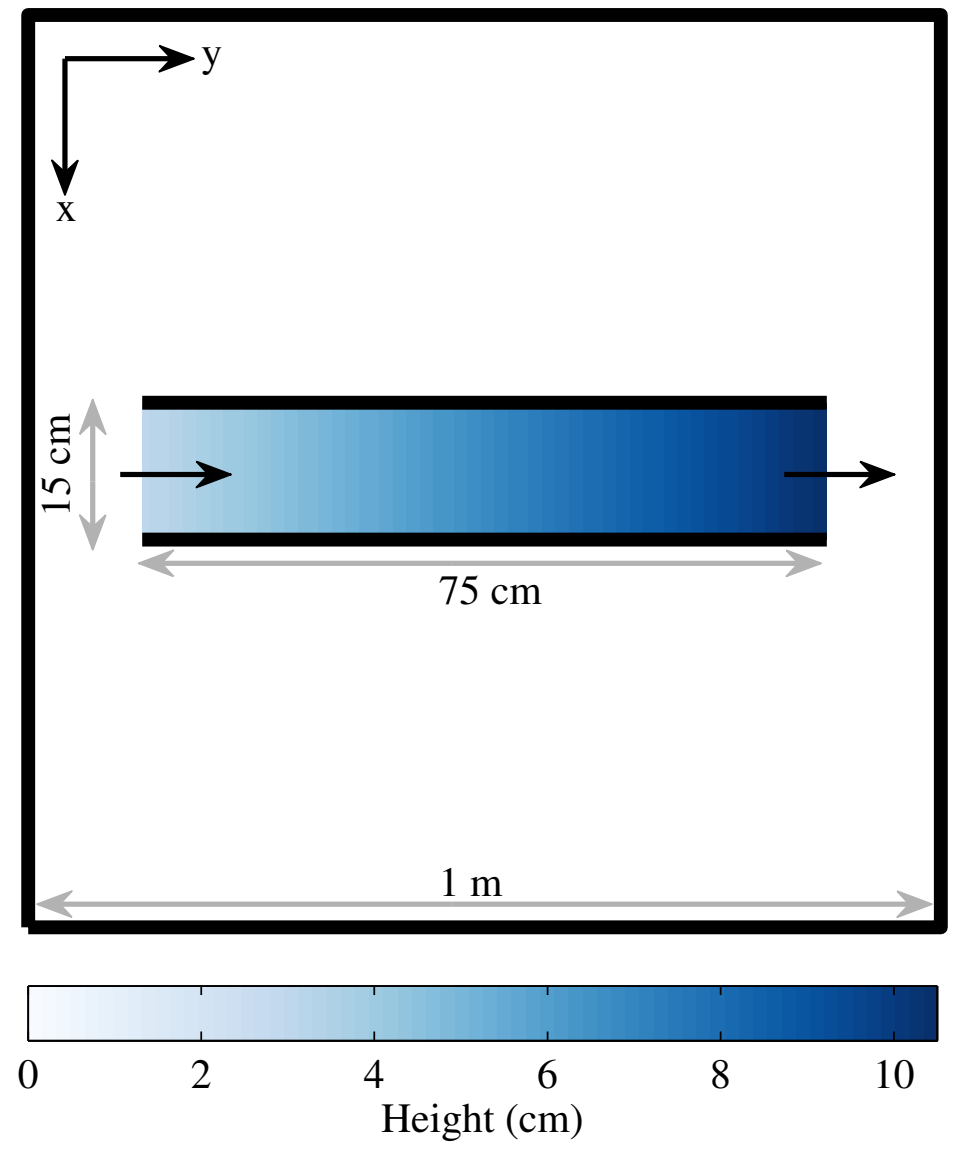

Figure 4-17: This schematic shows how a canyon experiment might be constructed on a standard $1 \mathrm{~m}$ tank on a turning table. The thick black lines are walls, the shaded blue area the sloping bottom topography, and the horizontal black arrows show the direction of mean flow through the canyon. 
of the canyon, but that would have to be done with great care, so as not to disrupt the stratification or the solid-body rotation. 


\section{Chapter 5}

\section{Consequences of Diffusion-Driven Boundary Layers}

We began this thesis by emphasizing the importance of knowing the mechanisms and distributions of abyssal mixing and upwelling for understanding the the ocean. This thesis proposed a mechanism - topographic interactions of diffusive boundary layers - that would have a profoundly different distribution of abyssal mixing than the internal wave based mechanisms that are most often invoked. Associated with that might be an importantly different abyssal circulation. In this brief conclusion, I will briefly review some of the novel contributions to physical oceanography made in this thesis, and then discuss some of the limitations of this work and some future directions that might address them. It is not yet possible to say with confidence whether the boundary layer mechanisms presented here are dynamically important, but they remain both interesting and promising.

\subsection{Novel Contributions of the Thesis}

Topography-driven exchange between the boundary layer and the far field. Perhaps the most important general result of this work is that exchanging water between the boundary layer and the far-field is about as difficult as falling off a log. Stratification is no barrier 
to water exchange when your boundary crosses density classes: water can and will move isopycnally between the boundary region and the far field. Since it need not gain or lose potential energy, substantial exchange can come from weak driving. Armi [1978] pointed this out, appealing to geostrophic eddies to provide an isopycnal velocity that would peel a boundary layer away from topography. As we saw in all three scientific chapters, though, even that is unnecessary: any change in the boundary layer dynamics will drive substantial exchanges. In two dimensions, changing the bottom angle $\alpha$ can change the local volume flux $Q$. In three dimensions, with a boundary layer in a canyon, the canyon walls are enough to continuously exchange the full volume of the viscous boundary layer with an overlying, much larger, geostrophic layer. Additional topography like sills can behave like two-dimensional topography, forcing water beyond the geostrophic layer into the far field. These exchange flows often carry as much volume as the boundary layer itself, in both two- and three-dimensions.

Broadly speaking, these exchanges allow the strong turbulence near the bottom to be communicated to the far field, affecting the stratification. Similarly, it also allows the boundary regions to import stratified water from the far field, and so to remain stratified even if there is significant stirring. No one questions that things like tides and eddies generate strong local turbulence when they pass over rough topography. There have been questions about whether that was important for the abyssal energy and mass budgets generally, or whether the boundary layers were relatively isolated from the rest of the water. I hope this work helps to settle the issue.

Overflows from boundary layer/topography interactions. This work demonstrates for the first time that a variety of different boundary layer geometries are possible from even very simple topography. For a wide variety of oceanographically-relevant parameter values, diffusive boundary layers will accelerate at a sill and form an overflow into the trough behind. It does not require external forcing. This means that such small-scale overflows may be ubiquitous in the deep ocean. We know from observations that large overflows, for example in the Romanche Fracture Zone [Polzin et al., 1996], are the sites 
of the most intense mixing observed in the deep ocean. There is no reason to believe that a boundary layer overflow would be anywhere near as energetic, but it probably would lead to a small but significant local increase in turbulent mixing. In the world's slow-spreading ridges, there are on the order of 10,000 sills obstructing canyons. If each is host to a small overflow, all of those small increases in turbulent mixing may add up to a dynamically important amount of mixing. They may help explain why microstructure measurements seem to show so much higher mixing in canyons than over the equally rough Mid-Atlantic Ridge flank.

In addition, in the non-rotating case we were able to accurately predict a priori what topography will produce an overflow and what will not. We did this by predicting the location of boundary layer separation, which was closely associated with the bottom stress at the sill of a bump or corrugation. Overflows tended to form when the sill height was similar to the boundary layer thickness and when the slope of the sills was similar to the background slope, as summarized in Figure 2-21. Though we had less success predicting the behavior in the two-dimensional rotating case, our problems came largely from $u_{\infty}$, the along-isobath velocity infinitely far from the boundary required by the two-dimensional solution. In canyons and other laterally-constricted flows, $u_{\infty}=0$, so the techniques developed in the non-rotating case may be more applicable.

In the two-dimensional rotating case, we were able to elucidate a set of trade-offs between Phillips-type behavior, which is buoyancy dominated and responds to the local slope, and Ekman-type behavior that is rotationally dominated and responds to the overall slope. In the steady-state case, the resulting exchange between the far field and the boundary layer scales with the slope Burger number, $S$, over almost two orders of magnitude. Though the long adjustment time for the uniform along-isobath flow means that the twodimensional rotating case is probably of limited applicability in the real ocean, this case was very valuable in understanding the three-dimensional circulation in a canyon.

Characterizing diffusion-driven flows in a canyon or channel. This work also gives the first description of the unforced circulation of stratified fluid in a channel with a 
sloping bottom. Instead of the two dynamical regimes seen in two dimensions-viscous bottom boundary layer and far field - the channel has four. Between the viscous layer and the far field is a geostrophic layer, where water that is forced out of the viscous layer when it reaches the right wall slowly and isopycnally circulates around the canyon and reenters the viscous layer on the left wall. Between the geostrophic layer and the bottom region is a corner region with mixed dynamics. The canyon walls require that all the water in the viscous layer be exchanged between the bottom layer and the geostrophic layer. In the Lagrangian frame, this manifests as water parcels moving along the channel in roughly helical trajectories. While in the viscous layer, the water parcel is transformed by mixing and moves along the canyon into a lighter density class; while in the geostraphic layer its water properties are constant.

All these trajectories show up in the Eulerian velocity field as two along-canyon jets, uniform in the along-canyon direction, whose size is set by the size of the corner region. One flows upslope, the other flows downslope, and they carry no net volume flux. Because of the geometry of the viscous layer, these jets are banked against their respective left wall. This overall circulation pattern provides several opportunities to compare this theory with real ocean circulations, for example by looking for along-canyon jets or by making a quantitative comparison with the along-canyon transport rates implied by tracer movement. Based on the size scaling for the jets derived in equation (4.17), we would expect the jets in a real canyon to be $0.5-5 \mathrm{~km}$ wide, depending on the local bottom slope.

Complexities of the boundary layer thickness. One of the themes to emerge from this work is the complexity of understanding the boundary layer thickness-how we define it, what mechanisms control it, and how we compare it to observations. The simplest definition of the boundary layer thickness is the scale provided by Phillips [1970] and Thorpe [1987]:

$$
\delta_{R}=\left(\frac{f^{2} \cos ^{2} \alpha_{0}}{4 \nu^{2}}+\frac{N^{2} \sin ^{2} \alpha_{0}}{4 \kappa \nu}\right)^{-1 / 4}
$$

This is the thickness over which isopycnals bend near a constant slope and also the thickness of the upslope bottom current. The boundary layer thickness increases with decreasing 
rotation or bottom slope, and with increasing viscosity or diffusivity. However, even using large eddy values for the viscosity and diffusivity, $\delta_{R}$ is tens of meters, not hundreds of meters. In observations, the easiest way to define the bottom boundary layer thickness is the distance over which isopycnals have a significant slope toward the bottom. This tends to be about $500 \mathrm{~m}$. The BBTRE current meter deployed by Toole [2007] found a significant upslope mean flow within 200-300 m of the bottom, consistent with the region of isopycnal tilt. Why is the observed boundary layer scale so much larger than the constant-slope Phillips/Thorpe boundary layer scale?

The work of this thesis provides a couple of potential mechanisms for answering that question. In Chapter 2, we saw how the troughs between adjacent sills tend to fill up with low stratification water, especially in cases where the boundary layer has overflows at the sills. In these cases, the boundary layer current is still confined within the Phillips/Thorpe boundary layer thickness, but the thickness defined by the isopycnal tilt is set by the height of the sills. The tallest sills in the $22^{\circ} \mathrm{S}$ canyon are about $500 \mathrm{~m}$ tall, which matches the region of isopycnal tilt.

In Chapter 4 we saw how introducing canyon walls lead to the formation of the geostrophic layer. This layer, which we expect to extend hundreds of meters from the bottom in a real canyon, has strong velocities but weak isopycnal slopes. It's possible that the strong upcanyon velocities and strong isopycnal slopes observed hundreds of meters from the bottom of the $22^{\circ} \mathrm{S}$ canyon could arise through some combination of the mechanisms seen in both of these chapters. It is also possible that there is an as-yet-unobserved bottom boundary layer whose scale matches the Phillips/Thorpe more closely. A 10-20 m layer could probably go undetected in the current observations. Therefore, it is unclear whether the $1.7 \mathrm{~cm} \mathrm{~s}^{-1}$ along-canyon velocity measured by Toole [2007] is in the Phillips layer, or in the geostrophic layer. 


\subsection{Limitations and Opportunities for Future Progress}

As with all interesting science problems (and the rest of life, one supposes), any effort is best considered a work in progress. There are a number of important limitations to the work, each of which can also be understood as an opportunity for future progress.

The first goal I outlined in the introduction - to better understand the basic physics of how stratified fluids interact with varying boundaries - has been advanced, but perhaps the greatest limitation to this work comes in the second goal outlined in the introduction. That goal was to apply that improved understanding to better understand how ocean-bottom topography affects the mixing, stratification, and transport of the ocean, especially in the context of abyssal canyons. Though this work proposes previously overlooked mechanisms for these processes, it is still very difficult to constrain their basin-scale impact quantitatively.

Even before starting this thesis, I could do the following back-of-the-envelope calculation for the total upwelling in diffusive boundary layers in the Brazil Basin. The volume flux constraint that arises from the heat flux divergence at the boundary in equation (1.13) tells us:

$$
Q=\kappa_{\infty} \cot \alpha
$$

If we take $\kappa_{\infty}$ to be the value from the base of the thermocline, it is about $\kappa_{\infty} \sim 10^{-5} \mathrm{~cm}^{2} \mathrm{~s}^{-1}$. A mean slope for the Mid-Atlantic Ridge flank canyons is about $\alpha_{0}=5 \times 10^{-3}$. If we multiply this by a typical canyon width $(L=25 \mathrm{~km})$ and the number of canyons in the Brazil Basin $(n)$, we get

$$
Q \sim \operatorname{Ln} \kappa_{\infty} \cot \alpha_{0} \sim 0.01 \mathrm{~Sv}
$$

This is much less than the measured inflow to the Brazil Basin through the Vema Channel of about $4 \mathrm{~Sv}$ [Hogg et al., 1982]. In order to supply $4 \mathrm{~Sv}$ through this mechanism, we would need $\kappa_{\infty}=4 \times 10^{-3}$, or approximately the local $\kappa$ observed in the canyons. However, though this is the net flux, the local flux inside a canyon can be much, much greater, as the local diffusivity is much greater. As we saw in the first appendix to Chapter 2, bottom 


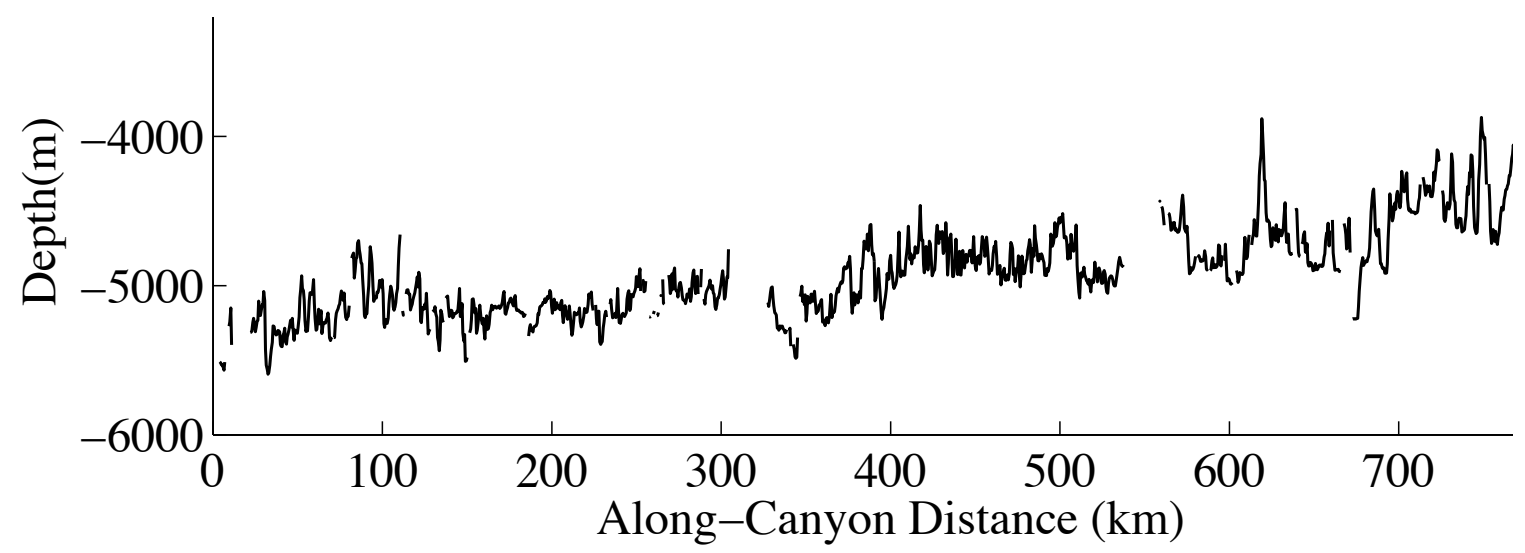

Figure 5-1: BBTRE $22^{\circ} \mathrm{S}$ canyon along-axis depth. It varies on many different scales.

boundary layers respond to the bottom $\kappa$. If $\kappa$ declines above, a compensating downslope flow develops above the boundary layer to satisfy the thermal constraint on $Q$. How that variation of viscosity and diffusivity with distance from the boundary will effect the mass transport in the boundary layer-which has no global constraint—remains an open question. It is a difficult one, as observations tell us that the scale over which the boundary effects are strong is the same scale that the diffusivity and viscosity vary over.

Similarly, we need to know how a boundary layer would interact with a realistic multiscale topography to assess the basin scale impact of the boundary layers. I've included here a plot of the along-axis depth of the $22^{\circ} \mathrm{S}$ canyon (Figure 5-1) to remind the reader just how little it resembles a perfectly periodic corrugation or a series of well-separated isolated sills. However unrealistic, though, it was exactly these kinds of simplificationsconstant diffusivity, single-scale topography - that allowed us to understand as much as we did of the boundary layers studied in this thesis.

There are many productive areas for future study to better understand these boundary layers and better constrain their role in the ocean. Nikurashin and Legg [2011] used synthetic topography with the same spectrum as the BBTRE study region topography for their study of the effects of internal tides. In the future, a similar method may work here. An intermediate step that would likely prove fruitful would be to look at the behavior of continuously varying topography in a canyon-corrugations instead of isolated sills. 
It would also be fascinating to understand the effects of time varying forcing, like tides. Umlauf and Burchard [2011] found transient static instability when they looked at tides over a constant slope. Introducing topography and varying its scale with respect to the tidal excursion parameter would likely produce even more evidence of topographic mixing.

Perhaps the most exciting avenues for future progress involve looking for these boundary layers outside of a numerical simulation. As discussed in the appendix to Chapter 2, the non-rotating work could very likely be tested at a laboratory scale. By using topography that was $\sim 10 \mathrm{~cm}$ long and millimeters high, we could probe the the same area on the regime diagram in Figure 2-21 that the oceanographic numerical simulations explore. It may even be possible to recreate the canyon circulation from Chapter 4 in the laboratory. Additionally, the study of circulation in an idealized canyon provides several hypotheses that could be tested observationally, like the existence of geostrophic jets banked against the left wall of the canyon and a reduced along-canyon transport from water parcels circulating in and out of the boundary layer. A tracer released in the bottom boundary layer might move continuously up the slope, or it might take the helical trajectory hypothesized in Chapter 4.

\subsection{Closing}

There is substantial and long-standing interest among physical oceanographers in the role of topography in abyssal mixing and so in abyssal transport and the abyssal energy budget. Most of that interest has focused on the role of internal waves, especially those generated by tides: their generation, propagation, and dissipation. In this thesis, I propose an alternate mechanism based on boundary layer dynamics for generating mixing and modifying the abyssal stratification through topography. This study is highly idealized, so it remains difficult to constrain the likely impact of this mechanism on a real ocean basin. However, the results suggest that by generating overflows and exchanges-both of which happen over a very wide range of conditions_-boundary layers may augment and distribute abyssal 
mixing. Many challenges remain before we can apply these ideas in global scale models, but the results are promising enough to make it worth the effort. 


\section{Bibliography}

M. R. Allshouse, M. F. Barad, and T. Peacock. Propulsion generated by diffusion-driven flow. Nature Physics, 6(516-519), 2010.

L. Armi. Some evidence for boundary mixing in the deep ocean. Journal of Geophysical Research, 83(C4):1971-1979, 1978.

J. A. Benthuysen and L. Thomas. Friction and diapycnal mixing at a slope: Boundary control of potential vorticity. Journal of Physical Oceanography, 42:1509-1523, 2012.

H. L. Bryden and A. J. G. Nurser. Effects of strait mixing on ocean stratification. Journal of Physical Oceanography, 33:1870-1872, 2003.

D. B. Chelton, R. A. deSzoeke, M. G. Schlax, K. El Naggar, and N. Siwertz. Geographical variability of the first-baroclinic rossby radius of deformation. Journal of Physical Oceanography, 28:433-460, 1998.

S. A. Condie. Ocean boundary mixing during Ekman layer arrest. Journal of Physical Oceanography, 29:2993-3001, 1999.

P. F. Cummins and M. G. G. Foreman. A numerical study of circulation driven by mixing over a submarine bank. Deep-Sea Research I, 45:745-769, 1998.

B. Cushman-Roisin. Introduction to Geophysical Fluid Dynamics. Academic Press, 2 edition, 2007.

R. W. Dell. Abyssal mixing from bottom boundary effects in Mid-Atlantic ridge flank canyons. Master's thesis, MIT/WHOI Joint Program, 2010.

K. Döös. Inter-ocean exchange of water masses. Journal of Geophysical Research, 100 (C7):13499-13514, 1995.

B. Ferron, H. Mercier, K. G. Speer, A. Gargett, and K. L. Polzin. Mixing in the romanche fracture zone. Journal of Physical Oceanography, 28:1929-1945, 1998.

A. C. Naveira Garabato, K. L. Polzin, B. A. King, K. J. Haywood, and M. Visbeck. Widespread intense turbulent mixing in the southern ocean. Science, 303:210-213, 2004. 
C. Garrett. The role of secondary circulation in boundary mixing. Journal of Geophysical Research, 95(C3):3181-3188, 1990.

C. Garrett. Marginal mixing theories. Atmosphere-Ocean, 29(2):313-339, 1991.

C. Garrett. Do near-boundary processes control the ocean? In Near-Boundary Processes and Their Parametrization: Proceedings of the 13th 'Aha Huliko'a Hawaiian Winter Workshop, 2003, pages 1-7, 2003.

C. Garrett and E. Kunze. Internal tide generation in the deep ocean. Annual Reviews of Fluid Mechanics, 39:57-87, 2007.

C. Garrett, P. MacCready, and P. B. Rhines. Arrested Ekman layers: Rotating stratified flow near a sloping boundary. Annual Reviews of Fluid Mechanics, 25:291-323, 1993.

M. C. Gregg. Diapycnal mixing in the thermocline. Journal of Geophysical Research, 92: 5249-5286, 1987.

N. G. Hogg, P. Biscaye, W. Gardner, and W. J. Schmitz, Jr. On the transport and modification of Antarctic Bottom Water in the Vema Channel. Journal of Marine Research, 40: 231-263 (suppl.), 1982.

J. R. Holton. The influence of viscous boundary layers on transient motions in a stratified rotating fluid. Journal of Atmospheric Science, 22:402-411, 1965.

Y. Hsueh. Buoyant Ekman layer. Physics of Fluids, 12:1757-1762, 1969.

R. X. Huang and X. Jin. Deep circulation in the south atlantic induced by bottom-intensified mixing over the midocean ridge. Journal of Physical Oceanography, 32:1150-1164, 2002.

G. N. Ivey. The role of boundary mixing in the deep ocean. Journal of Geophysical Research, 92:11873-11878, 1987.

G.H. Keller, S.H. Anderson, and J.W. Lavelle. Near-bottom currents in the mid-atlantic ridge rift valley. Canadian Journal of Earth Sciences, 12(4):703-710, 1975.

E. Kunze, L. K. Rosenfeld, G. S. Carter, and M. C. Gregg. Internal waves in Monterey submarine canyon. Journal of Physical Oceanography, 32:1890-1913, 2002.

J. R. Ledwell, A. J. Watson, and C. S. Law. Evidence for slow mixing across the pycnocline from an open-coean tracer-release experiment. Nature, 364:701-703, 1993.

J. R. Ledwell, E. T. Montgomery, K. L. Polzin, L. C. St. Laurent, R. W. Schmitt, and J. M. Toole. Evidence for enhanced mixing over rough topography in the abyssal ocean. Nature, 403:179 - 182, January 2000. 
P. MacCready and P. B. Rhines. Buoyant inhibition of Ekman transport on a slope and its effect on stratified spin-up. Journal of Fluid Mechanics, 223:631-661, 1991.

M. Y. Morris, M. M. Hall, L. C. St. Laurent, and N. G. Hogg. Abyssal mixing in the Brazil Basin. Journal of Physical Oceanography, 31:3331-33348, 2001.

W. H. Munk. Abyssal recipes. Deep-Sea Research, 13:707-730, 1966.

M. Nikurashin and S. Legg. A mechanism for local dissipation of internal tides generated at rough topography. Journal of Physical Oceanography, 41:378-395, 2011.

T. Peacock, R. Stocker, and J. M. Aristoff. An experimental investigation of the angular dependence of diffusion-driven flow. Physics of Fluids, 16(9):3503-3505, 2004.

O. M. Phillips. On flows induced by diffusion in a stably stratified fluid. Deep-Sea Research, 17:435-443, 1970.

O. M. Phillips, J.-H. Shyu, and H. Salmun. An experiment on boundary mixing: Mean circulation and transport rates. Journal of Fluid Mechanics, 173(473-499), 1986.

K. L. Polzin. An abyssal recipe. Ocean Modelling, 30:298-309, 2009.

K. L. Polzin, K. G. Speer, J. M. Toole, and R. W. Schmitt. Intense mixing of Antarctic bottom water in the equatorial Atlantic ocean. Nature, 380:54-57, March 1996.

K. L. Polzin, J. M. Toole, J. R. Ledwell, and R. W. Schmitt. Spatial variability of turbulent mixing in the abyssal ocean. Science, 276:93-96, April 1997.

A. F. Shchepetkin and J. C. McWilliams. The Regional Ocean Modeling System (ROMS): a split-explicit, free-surface, topography-following-coordinate oceanic model. Ocean Modelling, 9:347-404, 2005.

L. C. St. Laurent and A. M. Thurnherr. Intense mixing of lower thermocline water on the crest of the Mid-Atlantic Ridge. Nature, 448:680-683, August 2007.

L. C. St. Laurent, J. M. Toole, and R. W. Schmitt. Buoyancy forcing by turbulence above rough topography in the abyssal Brazil Basin. Journal of Physical Oceanography, 31: 3476-3495, December 2001a.

L. C. St. Laurent, J. M. Toole, and R. W. Schmitt. Mixing and diapycnal advection in the ocean. $2001 b$.

K. Stewartson. On almost rigid rotations. Journal of Fluid Mechanics, 3(1):17-26, 1957.

S. A. Thorpe. Current and temperature variability on the continental slope. Philosophical Transactions of the Royal Society London Series A, 323:471-517, 1987. 
A. M. Thurnherr and K. G. Speer. Boundary mixing and topographic blocking on the MidAtlantic Ridge in the South Atlantic. Journal of Physical Oceanography, 33:848-862, April 2003.

A. M. Thurnherr, L. C. St. Laurent, K. G. Speer, J. M. Toole, and J. R. Ledwell. Mixing associated with sills in a canyon on the midocean ridge flank. Journal of Physical Oceanography, 35:1370-1381, August 2005.

A. M. Thurnherr, G. Reverdin, P. Bouruet-Aubertot, L. C. St. Laurent, A. Vangriesheim, and V. Ballu. Hydrography and flow in the Lucky Strike segment of the Mid-Atlantic Ridge. Journal of Marine Research, 66(3):347-372, 2008.

J. M. Toole. Temporal characteristics of abyssal finescale motions above rough bathymetry. Journal of Physical Oceanography, 37:409-427, March 2007.

J. H. Trowbridge and S. J. Lentz. Asymmetric behavior of an oceanic boundary layer over a sloping bottom. Journal of Physical Oceanography, 21:1171-1185, 1991.

L. Umlauf and H. Burchard. Diapycnal transport and mixing efficiency in stratified boundary layers near sloping topography. Journal of Physical Oceanography, 41:329-345, 2011.

A. W. Woods. Boundary-driven mixing. Journal of Fluid Mechanics, 226:625-654, 1991.

C. Wunsch. On oceanic boundary mixing. Deep-Sea Research, 17:293-301, 1970.

C. Wunsch and R. Ferrari. Vertical mixing, energy, and the general circulation of the oceans. Annual Reviews of Fluid Mechanics, 36:281-314, 2004. 